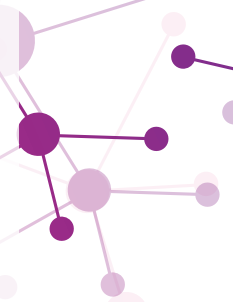





\section{Diet-induced \\ phenotypic plasticity during aging}

Fenni Rusli 


\section{Thesis Committee}

\section{Promotor}

Prof. Dr M.R. Müller

Professor of Nutrition, Metabolism and Genomics

Wageningen University

\section{Co-promotor}

Dr W.T. Steegenga

Assistant professor, Division of Human Nutrition

Wageningen University

\section{Other members}

Prof. Dr B.J. Zwaan, Wageningen University

Prof. Dr P.E. Slagboom, Leiden University Medical Center

Prof. Dr A.K. Groen, University of Groningen

Prof. Dr R. Shiri-Sverdlov, Maastricht University

This research was conducted under the auspices of the Graduate School VLAG

(Advanced studies in Food Technology, Agrobiotechnology, Nutrition and Health Sciences). 


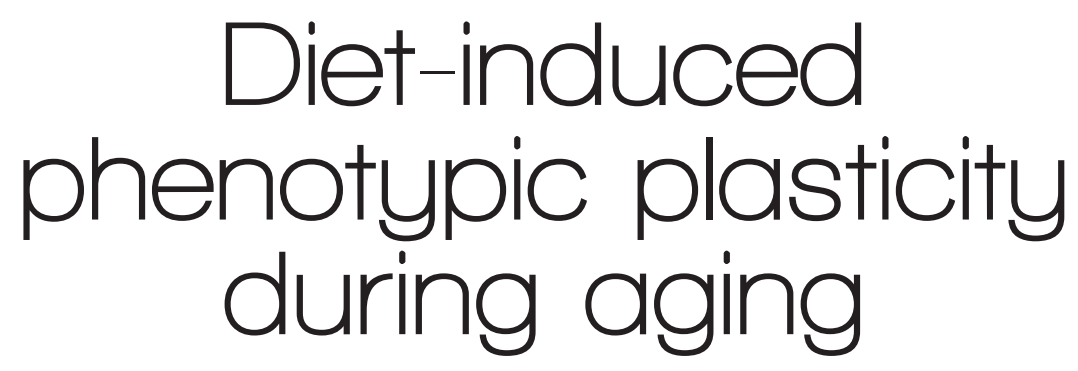

Fenni Rusli

Thesis

submitted in fulfilment of the requirements for the degree of doctor at Wageningen University

by the authority of the Rector Magnificus

Prof. Dr A.P.J. Mol,

in the presence of the

Thesis Committee appointed by Academic Board

to be defended in public

on Monday 29 August 2016

at 1.30 p.m. in the Aula. 
Fenni Rusli

Diet-induced phenotypic plasticity during aging, 180 pages.

$\mathrm{PhD}$ thesis, Wageningen University, Wageningen, NL (2016)

With references, with summary in English

ISBN: 978-94-6257-884-5

DOI $10.18174 / 387386$ 


\section{Contents}

\section{Chapter 1}

General introduction

\section{Chapter 2}

A weekly alternating diet between caloric restriction and medium-fat protects the liver from fatty liver development in middle-aged C57BL/6J mice

\section{Chapter 3}

Intermittent calorie restriction largely counteracts the adverse health effects of a medium-fat diet in aging C57BL/6J mice

\section{Chapter 4}

Plasticity of life-long calorie restricted C57BL/6 J mice in adapting to a medium-fat diet intervention at old age

\section{Chapter 5}

Fibroblast growth factor 21 reflects liver fat accumulation and dysregulation of signalling pathways in the liver of C57BL/6 J mice

\section{Chapter 6}

Discussion 



\section{Aging and diet}

In the past decades, multiple factors, in particular the drastically reduced mortality rate and the improvement of health care, have contributed to the aging population in developed countries and in many developing ones. The global share of older people (aged $>60$ years) has increased from $9.2 \%$ in 1990 to $11.7 \%$ in 2013 of the world population. In the future, the elderly population is expected to expand further, from 841 million in 2013 to more than 2 billion in 2050 (21.1\% of the world population) [1]. The increasing life expectancy goes hand in hand with the emergence of age-related chronic diseases and disabilities [2].

In humans, twin studies estimate that genetic differences have a modest contribution of $25 \%$ to lifespan variation in the population at large, underscoring environmental factors as the important contributor, $75 \%$ of the variation [3-6]. Since environment is a modifiable factor, it is crucial to understand how it affects the aging process, in order to identify healthy aging strategies. Among the components of environmental factors that influence aging, none is more instrumental than nutrition. In animal models, the most robust intervention that extends lifespan is calorie restriction (CR), a diet that reduces the amount of food intake by $30-40 \%$, which will be discussed in more details in the coming section. In the opposite situation, the current society's diets, which are typically energy-dense and high in (saturated) fat, sugars and digestible starch and low in dietary fibres and other plant food bioactives $[7,8]$, are directly associated with a worldwide outbreak of diseases $[9,10]$. The prevalence of these diseases, for example metabolic syndrome and non-alcoholic fatty liver disease, in general increases with age $[11,12]$.

\section{Aging liver}

The liver plays a key role in regulating metabolic health, which covers the metabolism of nutrients, xenobiotic, hormones and metabolic waste products, thereby maintaining homeostasis. The liver is central to these metabolisms, as much of the regulatory mechanisms are initiated in the liver, which then modulate metabolism activities of other organs [13]. To ensure homeostasis maintenance, the liver is equipped with a highly integrated and complementary network [14]. During aging functional and structural age-related impairments are observed in the liver, for example decreased liver volume, blood flow, and capacity for liver regeneration $[15,16]$. Such changes are associated with significant impairment of many hepatic metabolic and homeostatic activities with implications for systemic aging and age-related diseases. Aging liver is associated with a physiological increase of lipid accumulation [17]. Non-alcoholic fatty liver disease (NAFLD) is the most common chronic liver disease worldwide, which characteristic is the accumulation of fat $>5 \%$ in the liver [18]. NAFLD represents a spectrum of abnormalities from benign hepatic 
steatosis, non-alcoholic steatohepatitis (NASH) to liver cirrhosis and hepatocellular carcinoma. The different stages of NAFLD have been associated with aging, including hepatic steatosis [17, 19], steatohepatitis [20] and fibrosis [21, 22]. The mechanisms underlying the development of this complex disease are not fully understood, however NAFLD has been associated with molecular changes which are commonly observed during aging, e.g. reactive oxygen species formation, DNA damage, hepatocyte senescence and related epigenetic mechanisms [23-26].

Alterations in fat metabolism may lead to liver fat accumulation, i.e. increasing free fatty acid intake and de novo lipogenesis, decreasing fatty acid oxidation and hepatic very low density lipoprotein-triglyceride secretion [27]. A number of studies have highlighted the role of peroxisome proliferator-activated receptors a (PPARa) in NAFLD development [28-30]. PPARa is one of the isoform of PPAR transcription factor with the greatest affinity with fatty acid and the most abundant isotype in hepatocytes [31]. Due to its affinity to fatty acid, PPARa has a regulatory role in the adaptation to fed and fasted state. Its activation results in downstream transcription of genes involved in lipid metabolism, including fatty acid degradation, synthesis, transport, storage, lipoprotein metabolism and ketogenesis [32-34]. Moreover, PPARa regulates the expression of the fibroblast growth factor 21 (Fgf21) during starvation [35, 36]. In turn, Fgf21 acts as an endocrine hormone targeting various functions including metabolic control [37]. Studies characterising PPARa's role in aging has been limited, however, PPARa expression in the liver has been reported to decrease with age [38].

\section{Calorie restriction and aging}

Calorie restriction (CR), defined as a reduced energy intake without malnutrition, has been shown in numerous animal studies as by far the most effective approach to extend lifespan and prevent age-related diseases [39-41]. A 10-50\% reduction in calorie intake, compared to normal ad libitum consumption, induces a proportionate increase in maximum lifespan that is proportionate to the restriction degree $[42,43]$. Research on CR in humans has not been conclusive, but provides clues that the same beneficial metabolic adaptations that occur in model species are also observed in humans [42, 44]. Studies on humans voluntarily practicing long-term CR without malnutrition (i.e. Calorie Restriction with Optimal Nutrition society) showed advantages on cardiovascular health and features resembled those individuals 20 years younger on the typical Western diet $[45,46]$. A study on muscle transcriptome of the CR practitioners also supported the expression profile similarity to that of the younger subjects [44].

Despite the beneficial effects of a CR diet, this dietary regimen is rigorous and therefore difficult to be maintained for most humans. A previous study showed that after many years of habituation 
to a Western-style diet, the adaptation to CR for long-term is challenging [47]. Moreover, several serious health disadvantages of $\mathrm{CR}$ have been observed in humans and animal models: impairment of infection and wound healing, increased risk of impaired reproductive function, osteoporotic bone fractures, anemia and cardiac arrhythmias [48-50].

\section{Alternatives to CR dietary regimen}

Researchers in the nutrition and health science field continually search for a practicable and effective alternative to CR. Periodic CR (or even fasting), which applies CR at regular intervals in between periods of ad libitum food consumption has recently gained interest. This intermittent form of CR may improve the feasibility for people to maintain the dietary pattern. One of the difficulties of applying CR is impaired satiety, while the intermittent regimen allows a period when appetite can be satisfied. A number of dietary regimens that have been explored include alternate-day fasting (ADF), intermittent fasting (IF), intermittent starvation, every-other-day feeding and fasting mimicking diet (FMD). Studies exploring these dietary regimens have demonstrated promising results. Different research groups have shown that in humans and animal models ADF induces similar health benefits as CR, including increased lifespan and decreased risk of diabetes and cardiovascular disease [51-53]. Another variant, IF, has revealed greater resistance to endotoxic stress and improved insulin sensitivity [54]. Recently, Brandhorst et al. reported that the FMD extends life span in mice, reducing the incidence of cancer, boosting the immune system, reducing inflammatory diseases, slowing bone mineral density loss and improving the cognitive abilities in older mice. They extended the study to human subjects and found that this diet regimen decreases risk factors for aging, diabetes, cardiovascular disease and cancer [55]. It is important to realise that most of these dietary restriction variants employ a more severe food restriction (e.g. 75\% restriction in IF regimen) and the safety of the diet application has not been thoroughly examined [56]. Adverse effects of fasting include headache, fainting, weakness, dehydration, and hunger pangs. Fasting also slows the basal metabolic rate, so that the ad libitum consumption period could be more fattening after undergoing fasting. This implies that, the intermittent application of CR, instead of fasting, may provide less health risk, while still carries beneficial health outcomes.

\section{Phenotypic plasticity in aging}

Phenotypic plasticity is the ability to alter the phenotype to adaptively match to changes in the environment [57]. Physiological homeostasis, which means maintaining an equilibrium/balance in response to a varying environment by altering physiological parameters, also represents phenotypic plasticity [58]. In this context, aging can be viewed as a time-dependent loss of homeostatic 
integrity that maintain the structure and function of adult tissues [59]. The functional decline during aging implies a loss of the normal function or plasticity due to internal or external environmental signals. In the liver, for instance, the regenerative capacity to recover from insults has been shown to decreases at old age [60].

From an evolutionary perspective, our ancient ancestors' food supplies volatility most likely resulted in bouts of starvation periods. In the late Paleolithic era, during the time when humans existed as hunter-gatherers, cycle of feast and famine was not avoidable. This dietary pattern consists of oscillations in energy availability and therefore might have driven the selection of genes involved in the regulation of metabolism [61]. In the "thrifty genes" hypothesis, it is proposed that the energy availability oscillations result in natural selection of genes which favour efficient fat storage and use of energy. During the Paleolithic era the thrifty metabolism was likely beneficial for survival, but in the current times of permanent availability of foods this becomes a mismatch. From a metabolic perspective, chronic excessive energy loads may lead to a state of metabolic confusion, in which nutrient signalling pathways are constantly activated/inhibited. As a result, the gateways that control substrate traffic are never fully open or shut, and the continuous influx of carbon fuel from multiple sources and directions interferes with efficient substrate switching [62].

\section{STUDY AIMS AND DESIGN}

The Integrated Research on Developmental Determinants of Aging and Longevity (IDEAL) project aims to elucidate the role of early life conditions affecting late-life health, disease and aging. As a part of the IDEAL consortium, in 2011 we started a mice aging study in Wageningen University, aiming to investigate diet-induced phenotypic plasticity during aging. Specifically, the aim of the research presented in this thesis is to study the effects of various dietary interventions, including the novel intermittent calorie restriction (INT) diet, on shaping metabolic health and the transcriptomic profile of the liver during aging. We explored the effects of life-long exposure to INT diet, which is a weekly alternating dietary regimen between $40 \mathrm{E} \%$ CR and ad libitum medium-fat (MF) diets. This diet intervention challenges the body to adapt to fluctuations of energy availability and timerestricted feeding. We hypothesized that these continuous challenges to adapt to variations in the diet will offer the INT-exposed mice beneficial health effects. Three other diet groups were included in the study (Figure 1), which received either: 1) ad libitum control diet, 2) CR receiving 70E\% of the food consumed by the control group, or 3) ad libitum MF diet. The dietary intervention started at the age of 9 weeks and ended by the last sacrifice time point at 28 months. 


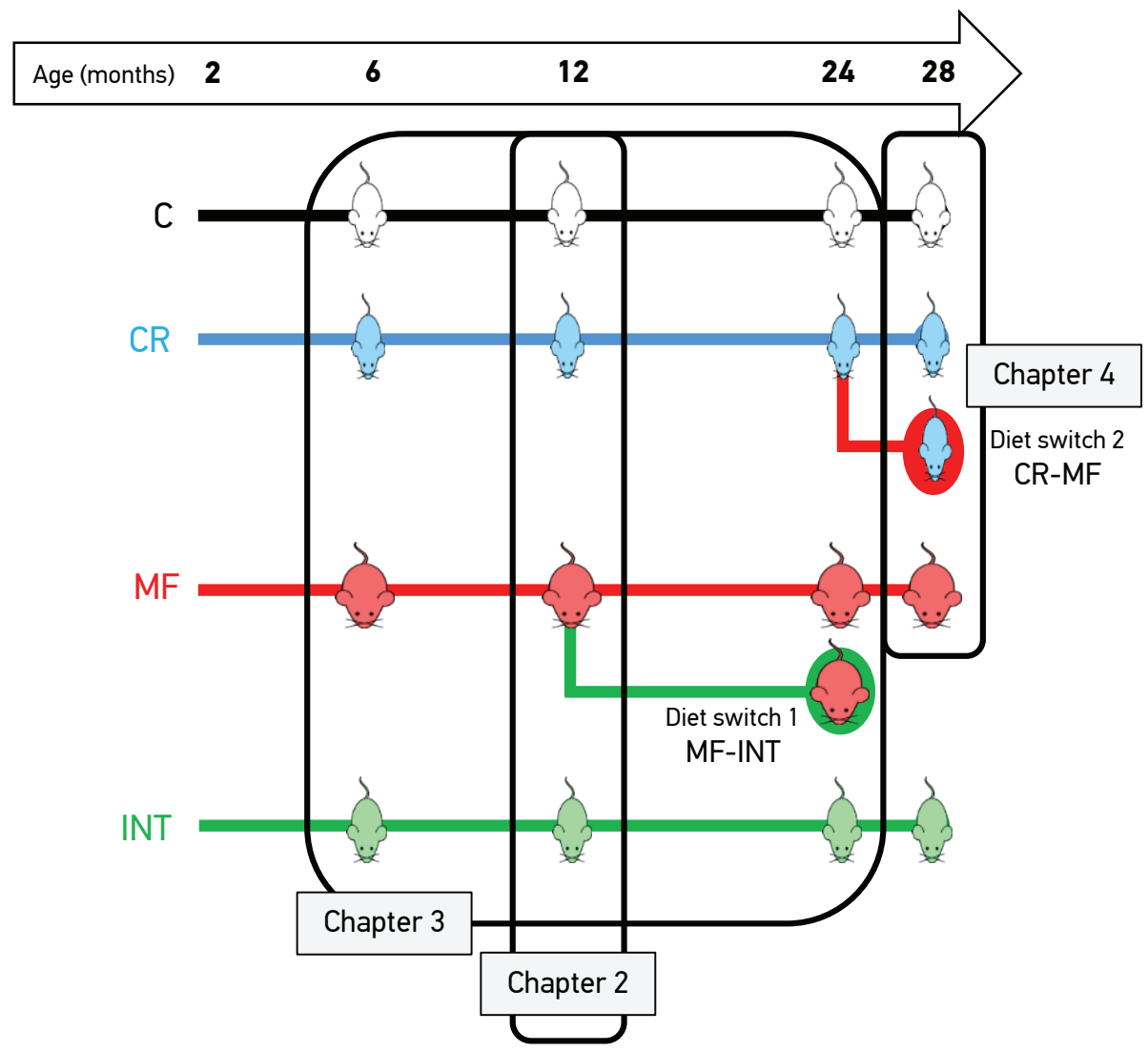

Figure 1. Study scheme of the mice aging cohort. At the age of 2 months, the mice were randomly assigned to either control (C), calorie restriction (CR), medium-fat (MF), or intermittent (INT) diet. The mice were culled at 4 different age time point: 6, 12, 24 and 28 months, which represent mature adult, middle age, old, and very old time point. The cohort also covered two diet switches: 1) MF to INT (MF-INT) diet switch at the age of 12 months and 2) CR to MF (CR-MF) diet switch at the age of 24 months. The boxes indicate the chapters that discuss the pointed diet and age groups.

Previous mice studies on NAFLD development typically apply a high percentage of fat (45$60 \mathrm{E} \%$ ) to resemble the Western-style diet [63]. However, NAFLD is acutely induced at young age and this does not represent the development in human populations, which accumulate the adverse effect of a Western-style diet over many years. In this study we applied a long-term exposure to a less extreme diet by using a fat content of $25 \mathrm{E} \%$ medium fat, which we expect to simulate a slow onset of NAFLD as it was observed in the Western human population. We chose the C57BL/6J mouse as the animal model, since metabolic features that are altered during the course of disease progression mirror the human situation [64].

Within the cohort, we incorporated two different diet switch experiments, which aimed to study the phenotypic plasticity of the mice at the middle and old age: 1) a switch at 12 months from 
continuous MF diet to INT diet, in order to explore INT diet as an obesity counteracting intervention, which was examined at the age of 24 months and 2) a switch at 24 months from continuous CR to ad libitum MF diet, to study the phenotypic plasticity of the life-long CR-exposed animals when challenged by changing environment to MF diet.

\section{OUTLINE OF THE THESIS}

In chapter 2, we investigated whether the INT diet is able to reverse the detrimental effects of a MF diet on the liver and its implication on NAFLD development. We showed that the INT diet maintained metabolic health and reversed the adverse effects of the MF diet, thus effectively prevented the development of NAFLD in middle-aged mice. The long term application of the INT diet regimen is further investigated in chapter $\mathbf{3}$, in which the mice were examined at an old age of 24 months. In this chapter, we also studied the response of the animals to a diet switch from continuous MF-exposure to an INT diet. Hereby we wanted to explore whether the INT regimen can reverse the long-term effects of MF diet in middle-aged mice. The adaptation to a diet switch at an old age is further investigated in chapter 4. A subset of mice that had been exposed to longterm CR for 22 months was switched to a MF diet and the responses at physiological, metabolic and gene expression levels were examined 4 months after the diet switch. In chapter $\mathbf{5}$, the data from the different dietary interventions and age time points were gathered to explore the molecular processes underlying the association of NAFLD and the plasma level of Fgf21, an emerging noninvasive biomarker for NAFLD. Finally, the general discussion and conclusions are presented in chapter 6. 


\section{REFERENCES}

1. United Nations Department of Economic and Social Affairs, Population Division World Population Aging Report. 2013 [cited 2016 January]; Available from: http://www.un.org/en/development/desa/population/ publications/pdf/ageing/WorldPopulationAgeing2013.pdf.

2. Niccoli, T. and Partridge, L., Ageing as a Risk Factor for Disease. Current Biology, 2012, 22,R741-R752.

3. Gurland, B.J., Page, W.F., and Plassman, B.L., A Twin Study of the Genetic Contribution to Age-Related Functional Impairment. The Journals of Gerontology Series A: Biological Sciences and Medical Sciences, 2004, 59,M859-M863.

4. Herskind, A.M., McGue, M., Holm, N.V., Sørensen, T.l., et al., The heritability of human longevity: a population-based study of 2872 Danish twin pairs born 1870-1900. Human genetics, 1996, 97,319-323.

5. Skytthe, A., Pedersen, N.L., Kaprio, J., Stazi, M.A., et al., Longevity Studies in GenomEUtwin. Twin Research, 2003, 6,448-454.

6. Eyre, H., Kahn, R., Robertson, R.M., et al., Preventing Cancer, Cardiovascular Disease, and Diabetes: A Common Agenda for the American Cancer Society, the American Diabetes Association, and the American Heart Association*†. CA: A Cancer Journal for Clinicians, 2004, 54,190-207.

7. Ledikwe, J.H., Blanck, H.M., Kettel Khan, L., Serdula, M.K., et al., Dietary energy density is associated with energy intake and weight status in US adults. The American Journal of Clinical Nutrition, 2006, 83,13621368.

8. Prentice, A.M. and Jebb, S.A., Fast foods, energy density and obesity: a possible mechanistic link. Obesity Reviews, 2003, 4,187-194.

9. Tiniakos, D.G., Vos, M.B., and Brunt, E.M., Nonalcoholic Fatty Liver Disease: Pathology and Pathogenesis. Annual Review of Pathology: Mechanisms of Disease, 2010, 5,145-171.

10. Fung, T.T., Rimm, E.B., Spiegelman, D., Rifai, N., et al., Association between dietary patterns and plasma biomarkers of obesity and cardiovascular disease risk. The American Journal of Clinical Nutrition, 2001, 73,61-67.

11. Aguilar, M., Bhuket, T., Torres, S., Liu, B., et al., PRevalence of the metabolic syndrome in the united states, 2003-2012. JAMA, 2015, 313,1973-1974.

12. Noureddin, M., Yates, K.P., Vaughn, I.A., Neuschwander-Tetri, B.A., et al., Clinical and histological determinants of nonalcoholic steatohepatitis and advanced fibrosis in elderly patients. Hepatology, 2013, 58,1644-1654.

13. Lebel, M., de Souza-Pinto, N.C., and Bohr, V.A., Metabolism, genomics, and DNA repair in the mouse aging liver. Current gerontology and geriatrics research, 2011, 2011,859415.

14. Timchenko, N.A., Aging and liver regeneration. Trends in Endocrinology \& Metabolism, 2009, 20,171-176.

15. Schmucker, D.L., Age-related changes in liver structure and function: Implications for disease ? Experimental Gerontology, 2005, 40,650-659.

16. Sheedfar, F., Biase, S.D., Koonen, D., and Vinciguerra, M., Liver diseases and aging: friends or foes? Aging Cell, 2013, 12,950-954.

17. Slawik, M. and Vidal-Puig, A.J., Lipotoxicity, overnutrition and energy metabolism in aging. Ageing Research Reviews, 2006, 5,144-164.

18. Bellentani, S., Scaglioni, F., Marino, M., and Bedogni, G., Epidemiology of Non-Alcoholic Fatty Liver Disease. Dig Dis, 2010, 28,155-161.

19. Petersen, K.F., Befroy, D., Dufour, S., Dziura, J., et al., Mitochondrial Dysfunction in the Elderly: Possible Role in Insulin Resistance. Science, 2003, 300,1140-1142.

20. Kichian, K., Mclean, R., Gramlich, L., Bailey, R., et al., Nonalcoholic fatty liver disease in patients investigated for elevated liver enzymes. Canadian Journal of Gastroenterology \& Hepatology, 2003, 17,38 - 42. 
21. Frith, J., Day, C.P., Henderson, E., Burt, A.D., et al., Non-Alcoholic Fatty Liver Disease in Older People. Gerontology, 2009, 55,607-613.

22. Argo, C.K., Northup, P.G., Al-Osaimi, A.M.S., and Caldwell, S.H., Systematic review of risk factors for fibrosis progression in non-alcoholic steatohepatitis. Journal of Hepatology, 2009, 51,371-379.

23. Hoare, M., Das, T., and Alexander, G., Ageing, telomeres, senescence, and liver injury. Journal of Hepatology, 2010, 53,950-961.

24. López-Otín, C., Blasco, M.A., Partridge, L., Serrano, M., et al., The Hallmarks of Aging. Cell, 2013, 153,11941217.

25. Mann, D.A., Epigenetics in liver disease. Hepatology, 2014, 60,1418-1425.

26. Jin, J., Wang, G.-L., lakova, P., Shi, X., et al., Epigenetic changes play critical role in age-associated dysfunctions of the liver. Aging Cell, 2010, 9,895-910.

27. Tilg, H. and Moschen, A.R., Evolution of inflammation in nonalcoholic fatty liver disease: The multiple parallel hits hypothesis. Hepatology, 2010, 52,1836-1846.

28. Abdelmegeed, M.A., Yoo, S.-H., Henderson, L.E., Gonzalez, F.J., et al., PPARa Expression Protects Male Mice from High Fat-Induced Nonalcoholic Fatty Liver. The Journal of Nutrition, 2011, 141,603-610.

29. Staels, B., Rubenstrunk, A., Noel, B., Rigou, G., et al., Hepatoprotective effects of the dual peroxisome proliferator-activated receptor alpha/delta agonist, GFT505, in rodent models of nonalcoholic fatty liver disease/nonalcoholic steatohepatitis. Hepatology, 2013, 58,1941-1952.

30. Montagner, A., Polizzi, A., Fouché, E., Ducheix, S., et al., Liver PPARa is crucial for whole-body fatty acid homeostasis and is protective against NAFLD. Gut, 2016.

31. Desvergne, B., Michalik, L., and Wahli, W., Transcriptional Regulation of Metabolism. Physiol Rev, 2006, 86.

32. Kersten, S., Seydoux, J., Peters, J.M., Gonzalez, F.J., et al., Peroxisome proliferator-activated receptor a mediates the adaptive response to fasting. J Clin Invest, 1999, 103,1489-1498.

33. Rakhshandehroo, M., Sanderson, L.M., Matilainen, M., Stienstra, R., et al., Comprehensive Analysis of PPARa-Dependent Regulation of Hepatic Lipid Metabolism by Expression Profiling. PPAR Research, 2007, 2007.

34. Kersten, S., Integrated physiology and systems biology of PPARa. Molecular Metabolism, 2014, 3,354-371.

35. Badman, M.K., Pissios, P., Kennedy, A.R., Koukos, G., et al., Hepatic fibroblast growth factor 21 is regulated by PPARa and is a key mediator of hepatic lipid metabolism in ketotic states. Cell metabolism, 2007, 5,426-437.

36. Inagaki, T., Dutchak, P., Zhao, G., Ding, X., et al., Endocrine regulation of the fasting response by PPARamediated induction of fibroblast growth factor 21. Cell metabolism, 2007, 5,415-425.

37. Kharitonenkov, A. and Larsen, P., FGF21 reloaded: challenges of a rapidly growing field. Trends in Endocrinology \& Metabolism, 2011, 22,81-86.

38. Sanguino, E., Roglans, N., Alegret, M., Sánchez, R.M., et al., Atorvastatin reverses age-related reduction in rat hepatic PPARa and HNF-4. British Journal of Pharmacology, 2005, 145,853-861.

39. Spindler, S.R., Caloric restriction: From soup to nuts. Ageing Research Reviews, 2010, 9,324-353.

40. de Cabo, R., Carmona-Gutierrez, D., Bernier, M., Hall, Michael N., et al., The Search for Antiaging Interventions: From Elixirs to Fasting Regimens. Cell, 2014, 157,1515-1526.

41. Fontana, L. and Partridge, L., Promoting Health and Longevity through Diet: From Model Organisms to Humans. Cell, 2015, 161,106-118.

42. Fontana, L., Partridge, L., and Longo, V.D., Extending Healthy Life Span—From Yeast to Humans. Science, 2010, 328,321-326.

43. Masoro, E.J., Overview of caloric restriction and ageing. Mechanisms of Ageing and Development, 2005, 126,913-922.

44. Mercken, E.M., Crosby, S.D., Lamming, D.W., JeBailey, L., et al., Calorie restriction in humans inhibits the PI3K/AKT pathway and induces a younger transcription profile. Aging Cell, 2013, 12,645-651. 
45. Meyer, T.E., Kovács, S.J., Ehsani, A.A., Klein, S., et al., Long-Term Caloric Restriction Ameliorates the Decline in Diastolic Function in Humans. Journal of the American College of Cardiology, 2006, 47,398-402.

46. Stein, P.K., Soare, A., Meyer, T.E., Cangemi, R., et al., Caloric restriction may reverse age-related autonomic decline in humans. Aging Cell, 2012, 11,644-650.

47. Racette, S.B., Weiss, E.P., Villareal, D.T., Arif, H., et al., One Year of Caloric Restriction in Humans: Feasibility and Effects on Body Composition and Abdominal Adipose Tissue. The Journals of Gerontology Series A: Biological Sciences and Medical Sciences, 2006, 61,943-950.

48. Dirks, A.J. and Leeuwenburgh, C., Caloric restriction in humans: Potential pitfalls and health concerns. Mechanisms of Ageing and Development, 2006, 127,1-7.

49. Kristan, D.M., Calorie restriction and susceptibility to intact pathogens. AGE, 2008, 30,147-156.

50. Hunt, N.D., Li, G.D., Zhu, M., Levette, A., et al., Effect of calorie restriction and refeeding on skin wound healing in the rat. AGE, 2011, 34,1453-1458.

51. Varady, K.A., Roohk, D.J., Loe, Y.C., McEvoy-Hein, B.K., et al., Effects of modified alternate-day fasting regimens on adipocyte size, triglyceride metabolism, and plasma adiponectin levels in mice. Journal of Lipid Research, 2007, 48,2212-2219.

52. Varady, K.A., Hudak, C.S., and Hellerstein, M.K., Modified alternate-day fasting and cardioprotection: relation to adipose tissue dynamics and dietary fat intake. Metabolism, 2009, 58,803-811.

53. Bhutani, S., Klempel, M.C., Berger, R.A., and Varady, K.A., Improvements in Coronary Heart Disease Risk Indicators by Alternate-Day Fasting Involve Adipose Tissue Modulations. Obesity, 2010, 18,2152-2159.

54. Anson, R.M., Guo, Z., de Cabo, R., lyun, T., et al., Intermittent fasting dissociates beneficial effects of dietary restriction on glucose metabolism and neuronal resistance to injury from calorie intake. Proceedings of the National Academy of Sciences, 2003, 100,6216-6220.

55. Brandhorst, S., Choi, In Y., Wei, M., Cheng, Chia W., et al., A Periodic Diet that Mimics Fasting Promotes Multi-System Regeneration, Enhanced Cognitive Performance, and Healthspan. Cell Metabolism, 2015, 22,86-99.

56. Horne, B.D., Muhlestein, J.B., and Anderson, J.L., Health effects of intermittent fasting: hormesis or harm? A systematic review. The American Journal of Clinical Nutrition, 2015, 102,464-470.

57. DeWitt, T.J. and Scheiner, S.M., Phenotypic plasticity : functional and conceptual approaches. 2004, New York [etc.]: Oxford University Press.

58. Whitman, D. and Agrawal, A., What is phenotypic plasticity and why is it important?, in Phenotypic Plasticity of Insects: Mechanisms and Consequences, W. DW and A. TN, Editors. 2009, Science Publishers: Enfield. p. $1-63$.

59. Rando, Thomas A. and Chang, Howard Y., Aging, Rejuvenation, and Epigenetic Reprogramming: Resetting the Aging Clock. Cell, 2012, 148,46-57.

60. Gan, L., Chitturi, S., and Farrell, G.C., Mechanisms and Implications of Age-Related Changes in the Liver: Nonalcoholic Fatty Liver Disease in the Elderly. Current Gerontology and Geriatrics Research, 2011, $2011,12$.

61. Chakravarthy, M.V. and Booth, F.W., Eating, exercise, and "thrifty" genotypes: connecting the dots toward an evolutionary understanding of modern chronic diseases. Journal of Applied Physiology, 2004, 96,3-10.

62. Muoio, Deborah M., Metabolic Inflexibility: When Mitochondrial Indecision Leads to Metabolic Gridlock. Cell, 2014, 159,1253-1262.

63. Buettner, R., Schölmerich, J., and Bollheimer, L.C., High-fat Diets: Modeling the Metabolic Disorders of Human Obesity in Rodents. Obesity, 2007, 15,798-808.

64. Collins, S., Martin, T.L., Surwit, R.S., and Robidoux, J., Genetic vulnerability to diet-induced obesity in the C57BL/6J mouse: physiological and molecular characteristics. Physiology \& Behavior, 2004, 81,243-248. 




\section{ABSTRACT}

Scope: We aimed to investigate whether a novel dietary intervention consisting of an every-other-week calorie restricted diet could prevent non-alcoholic fatty liver disease (NAFLD) development induced by a medium-fat diet.

Methods and results: Nine week-old male C57BL/6J mice received either a 1) control (C), 2) $30 \mathrm{E} \%$ calorie restricted (CR), 3) medium-fat (MF; 25E\% fat) or 4) intermittent (INT) diet, a diet alternating weekly between $40 \mathrm{E} \% \mathrm{CR}$ and an ad libitum MF diet until sacrifice at the age of 12 months. The metabolic, morphological, and molecular features of NAFLD were examined. The INT diet resulted in healthy metabolic and morphological features as displayed by the continuous CR diet: glucose tolerant, low hepatic triglyceride content, low plasma alanine aminotransferase. In contrast, the C- and MF-exposed mice with high body weight developed signs of NAFLD. However, the gene expression profiles of INT-exposed mice differed to those of CR-exposed mice and showed to be more similar with those of C- and MF-exposed mice with a comparable body weight.

Conclusions: Our study reveals that the INT diet maintains metabolic health and reverses the adverse effects of the MF diet, thus effectively prevent the development of NAFLD in 12-month-old male C57BL/6J mice. 


\section{INTRODUCTION}

The modern Western-style diet and sedentary lifestyle often promote a positive energy balance, which has importantly contributed to the global rapid increase in the prevalence of metabolic syndrome in the recent decades [1-4]. Non-alcoholic fatty liver disease (NAFLD), a condition in which the liver excessively accumulates fat, has been considered as a hepatic manifestation of metabolic syndrome. Therefore, the increasing prevalence of metabolic syndrome and NAFLD becomes a major concern $[5,6]$ and implies an urgent need for a feasible and effective dietary intervention to prevent NAFLD [7].

Calorie restriction is widely known for its beneficial effects on health that is consistently demonstrated in various species [8-10], and these beneficial effect also include a decrease in hepatic triglycerides level $[11,12]$. However, an issue that has been frequently raised is the adherence of the general population to such a strict eating pattern [13,14], as many individuals might encounter difficulties on long-term adaptation to CR after many years of habituation to a Western-style diet, as was shown by Racette et al. [15]. Therefore, a dietary regimen with alternating applications of food restriction or even food abstinence and ad libitum consumption emerges as an attractive option. Various forms of alternating dietary intervention have been explored, such as alternateday fasting, intermittent fasting, intermittent starvation and every-other-day feeding. The results are promising, the health benefits of the different alternative regimens are similar to continuous exposure to calorie restriction, including improved glucose tolerance and decreased cardiovascular disease risk [14, 16-19]. Consequently, the application of an alternating dietary regimen as a preventive measurement against the development of NAFLD becomes of interest.

In this study, we aimed to investigate whether an every-other-week restricted diet, which we termed as the intermittent (INT) diet, is able to reverse the detrimental effects of a Western-style diet on the liver and its implication on NAFLD development in the male C57BL/6 J mice. In the INT diet we applied a medium-fat (MF; 25E\% fat) ad libitum diet to represent the Western-style diet and a $40 \mathrm{E} \%$ calorie restriction (CR) diet as the restricted diet, and the animal feeding was alternated in a weekly basis between the MF and 40E\% CR. To demonstrate a progressive NAFLD development in the Western-style diet, we included a diet group of continuous MF diet ad libitum. In addition, we also included two other diet groups, an ad libitum feeding of a control diet (C) and a continuous $30 \mathrm{E} \% \mathrm{CR}$ to complement the comparison to a normal and healthy diet respectively.

We demonstrated that the INT diet resulted in proper glucose tolerance, low hepatic triglyceride content, low plasma alanine aminotransferase, similarly to the continuous CR diet. On the other hand, the C- and MF-exposed mice with high body weight developed signs of NAFLD. However, the INT diet group did not share much similarities on the gene expression level with the CR diet 
group. The gene expression of INT-exposed mice showed to be more comparable with those of C- and MF-exposed mice with a comparable body weight. Thus, the INT diet effectively prevents the development of NAFLD in C57BL/6J mice, it maintains healthy physiological features without altering the gene expression profile.

\section{MATERIALS AND METHODS}

\section{Ethics statement}

Experiments were approved by the Local Committee for Care and Use of Laboratory Animals at Wageningen University (code number: drs-2010151b).

\section{Animals and diets}

Male C57BL/6 J mice (age of 7 weeks) were purchased from Janvier (Cedex, France) and were housed in pairs of two in the light and temperature $\left(20^{\circ} \mathrm{C}\right)$-controlled animal facility of Wageningen University (12-hour light/dark cycle, light on at 04.00). The mice received standard AIN-93G diet [20] (Research Diet Services, Wijk bij Duurstede, The Netherlands) for 2 weeks upon arrival.

At the start of the diet intervention the mice were 9 weeks old, housed individually and randomly distributed into four intervention groups: 1) Control diet (C) receiving AIN-93W diet ad libitum ( $\mathrm{n}=89$ ); 2) calorie restricted diet (CR) receiving AIN-93W-CR in portions containing 70E\% of the mean energy intake (30E\% reduced energy intake) of the group of the control mice were provided each day at 15.30 ( $n=117)$; 3) medium fat diet (MF; 25E\% fat) receiving AIN-93W-MF ad libitum ( $\mathrm{n}=127$ ); and (4) intermittent diet (INT) receiving alternating one week AIN-93W-MF ad libitum followed by one week $60 \mathrm{E} \%$ based on the mean energy intake (40E\% reduced energy intake) of the mice on the AIN-93W diet ( $n=155)$. AIN-93W is a variant of AIN-93M (maintenance of adult mice), which slightly differs on the fat source. The $10 \mathrm{E} \%$ fat content in AIN-93M solely comes from soybean oil, while the fat source of AIN-93W is a mix of $6 \mathrm{E} \%$ fat soybean oil and $4 \mathrm{E} \%$ palm oil, in order to balance saturated and unsaturated fat composition. AIN-93W-CR contained increased concentration of vitamins and minerals content in order to feed these mice the same concentrations of micronutrients as the mice receiving AIN-93W diet and avoid malnutrition. Complete diet composition is listed in Supplementary Table 1 (Research Diet Services, Wijk bij Duurstede, The Netherlands). All mice were provided with ad libitum access to water.

Body weight of all mice was recorded every two weeks. To represent a weekly body weight development, we weighed a smaller sample of mice of each intervention group every other week 
(20-24 mice). Food intake of 20 mice of each intervention group was measured every three months, comprising one week measurement for the C, CR and MF-fed mice and two weeks measurement for the INT fed mice. Portion sizes of the mice on the CR and INT were adjusted at the beginning of the study and at the age of 6 months based on food intake of $C$ mice.

At the age of 12 months, 14 mice of each intervention group were sacrificed between 14.0017.00 on 5 consecutive days (the remaining mice stayed in the experiment to allow an investigation at older ages). To analyse an adaptive capacity of peroxisome proliferator-activated receptor alpha (PPARa), 7 mice in each intervention group were treated with a PPARa agonist, while the rest of the animals were mock-treated. Prior to sacrifice each mouse was first fasted for 4 hours after which they received an intragastric gavage of either solvent $(0.5 \%$ carboxymethyl cellulose) or PPARa agonist Wy-14,643 dispersed in solvent (160 mg Wy-14,643/kg body weight), then fasted again for another 6 hours. All 14 mice of each diet group were included in metabolic parameter measurements to allow more optimal statistical analyses, but only 7 mock-treated animals were included in molecular analysis, since the treatment with the PPARa agonist would affect the gene expression levels. The PPARa adaptive capacity analysis will be covered in a separate publication. INT mice were sacrificed in their ad libitum MF feeding week.

After sedation with a mixture of isoflurane (1.5\%), nitrous oxide (70\%) and oxygen (30\%), blood samples were collected by cardiac puncture, then followed by neck dislocation. The epidydimal white adipose tissue (WAT) and liver were weighed and were subsequently snap-frozen and stored at $-80^{\circ} \mathrm{C}$ until further molecular/biochemical analysis. For histological analysis, the livers were fixed in $4 \%$ paraformaldehyde.

\section{Oral glucose tolerance test}

The mice sacrificed at the age of 12 month were all subjected to an oral glucose tolerance test (OGTT) two weeks prior to sacrifice. In the OGTT, the mice were fasted for 6 hours, then received 1.5 $\mathrm{mg}$ glucose per gram body weight via an oral gavage. Subsequently, blood glucose was measured 15, 30, 45, 60, 90 and 150 minutes following the glucose load using Accu-Check blood glucose meters (Roche Diagnostics, Almere, The Netherlands).

\section{RNA isolation}

Total RNA was isolated using TRIzol reagent (Invitrogen, Breda, The Netherlands) according to the manufacturer's instructions. The RNA was treated with DNAse and purified on columns using the RNeasy microkit (Qiagen, Venlo, The Netherlands). RNA concentration was measured on a NanoDrop ND-1000 UV-vis spectrophotometer (Isogen, Maarsen, The Netherlands) and 
RNA integrity was checked on an Agilent 2100 Bioanalyzer (Agilent Technologies, Amsterdam, The Netherlands) with 6000 Nano Chips according to the manufacturer's instructions. RNA was judged as suitable only if samples showed intact bands of $18 \mathrm{~S}$ and $28 \mathrm{~S}$ ribosomal RNA subunits, displayed no chromosomal peaks or RNA degradation products, and had a RNA integrity number (RIN) above 8.0.

\section{Microarray hybridization and analysis}

To reveal the liver transcriptomic profile of the four different diets, whole-genome gene expression was analysed by microarray analysis. This analysis included 7 mock-treated animals from each C, MF and INT diet groups, and 6 animals from the CR diet group. One hundred nanogram of RNA was used for Whole Transcript cDNA synthesis (Affymetrix, Santa Clara, CA, USA). Hybridization, washing and scanning of Affymetrix GeneChip Mouse Gene 1.1 ST arrays were carried out according to standard Affymetrix protocols. Arrays were normalized using the Robust Multiarray Average method [21, 22]. Probe sets were defined according to Dai et al. [23]. In this method probes are assigned to unique gene identifiers, in this case Entrez IDs. The probes on the Gene 1.1 ST arrays represent 21,225 Entrez IDs. For the analysis, only genes having intensity value of $>20$ on at least 5 array were taken into account, which resulted in 14,758 genes. Array data have been submitted to the Gene Expression Omnibus, with accession number GSE61233. The hierarchical clustering plot depicting gene expression profile similarity was constructed by using Multiple Experiment Viewer [24] and the accompanying body weight heatmap was prepared in Excel.

\section{cDNA synthesis and real-time quantitative PCR}

Real-time quantitative PCR (Q-PCR) was used to quantify gene expression changes for a selection of genes on all individual samples, as described previously [25]. For each individual sample, single-stranded complementary DNA (cDNA) was synthesized from $1 \mu \mathrm{g}$ of total RNA using the First Strand cDNA Synthesis Kit (Thermo Scientific, Landsmeer, The Netherlands) following the supplier's protocol. Primer sequences were retrieved from the online PrimerBank database [26], or otherwise designed using the Primer3 program [27] and the sequences of the primers used are listed in Supplementary Table 2. Primers were tested for specificity by BLAST analysis. Q-PCR was performed using SensiMix SYBR No-ROX kit (Bioline, Alphen aan den Rijn, The Netherlands) and CFX384 thermal cycler (Bio-Rad, Veenendaal, The Netherlands). The following thermal cycling conditions were used: 2 min at $94^{\circ} \mathrm{C}$, followed by 40 cycles of $94^{\circ} \mathrm{C}$ for $15 \mathrm{~s}$ and $60^{\circ} \mathrm{C}$ for $45 \mathrm{~s}$. Q-PCR reactions were performed in duplicate and all samples were normalized to $18 \mathrm{~S}$ expression. 


\section{Histology}

$5 \mu \mathrm{m}$ paraffin-embedded sections were cut and mounted on Superfrost microscope slides. The sections were de-waxed in xylene and rehydrated in a series of graded alcohols. After staining with Meyer's hematoxylin-eosine (H-E) or fast green FCF/Sirius red F3B staining sections were mounted with DePex mounting medium (Gurr, BDH, Poole, Dorset, UK).

\section{Hepatic triglyceride content determination}

Liver homogenates of $5 \% \mathrm{w} / \mathrm{v}$ were prepared in buffer containing $250 \mathrm{mM}$ sucrose, $1 \mathrm{mM}$ EDTA, $10 \mathrm{mM}$ Tris- $\mathrm{HCl}$ (pH 7.5). Liver triglyceride content was determined using the Triglyceride Liquicolor Monoreagent from Instruchemie (Delfzijl, The Netherlands) according to the manufacturer's instruction.

\section{Plasma measurement}

Plasma concentration of ALT and AST were measured with commercially available kits from Instruchemie (Delfzijl, the Netherlands) following the protocol optimized by Stienstra et al. [28]. Plasma insulin level was measured using kit from ALPCO Diagnostics (Salem, NH, USA) according to the manufacturer's instruction.

\section{Statistical analysis}

Data analysis was performed with GraphPad Prism version 5.04 (GraphPad Software, San Diego, USA), using one-way ANOVA followed by Tukey post-test analysis or Kruskal-Wallis test followed by a Dunn's post-test for not normally-distributed data. Correlation analysis was performed using Pearson correlation. $P<0.05$ was considered significant. 


\section{RESULTS}

\section{Modest body, liver and WAT weight gain in mice exposed to an intermittent diet}

To determine the physiological features of mice after exposure to an INT diet regimen, various parameters were measured. Adaptation to the high/low energy intake was observed in the weekly body weight measurements of the INT-fed mice, showing fluctuations in mean body weight related to the diet received in the preceding week (Fig. 1A). After the ad libitum feeding week body weight of the INT mice was lower than that of $\mathrm{C}$ and MF groups, while after the calorie restricted week their body weight remained higher than that of the mice in the CR group. Strong heterogeneity in body weight of mice in the $C$ and MF diet groups was observed, which was reflected by the large error bars, in contrast to the mice within the CR and INT groups where the variation was very low.

Body weight gain in C- and MF-fed mice included a significant increase in adiposity measured by the amount of white adipose tissue present in WAT, compared to the CR- and INT-fed mice $(P<0.001$, Fig. 1B). Similarly, the WAT-to-body weight ratio also showed a significant increase in $C$ and MF $(P<0.001)$. Liver weights of the INT-fed mice were nearly as low as that of the CR mice and differed significantly from the liver weights of the $C$ and MF groups $(P<0.05$ and $<0.001$, respectively, Fig. 1C). Interestingly, while the liver-to-body weight ratio of the $C, C R$ and MF groups were comparable, a slight but significant decrease was found for the INT-fed mice compared to CR and MF exposed mice $(P<0.05)$. Overall, a strong correlation between liver and body weight was observed $(r=0.83)$ and also between liver and WAT weight $(r=0.82)$. 
A
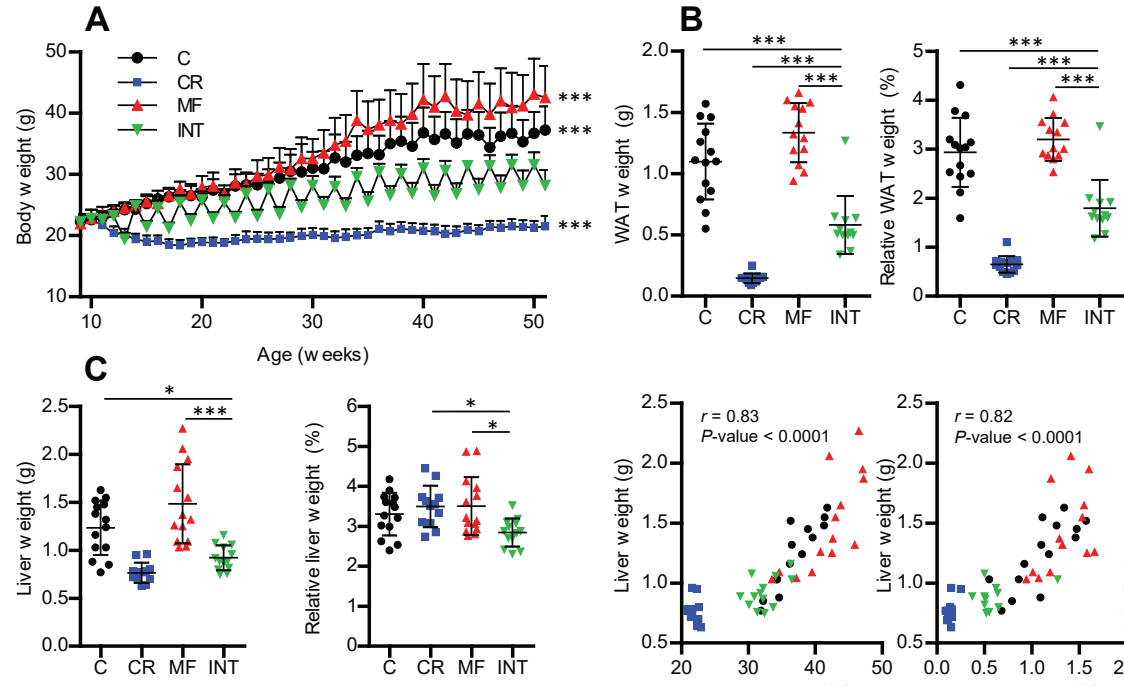

D

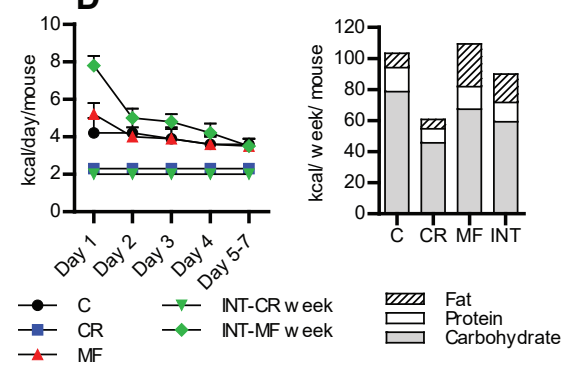

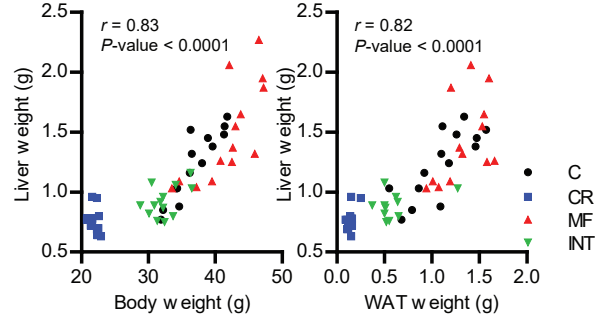

$\mathbf{F}$
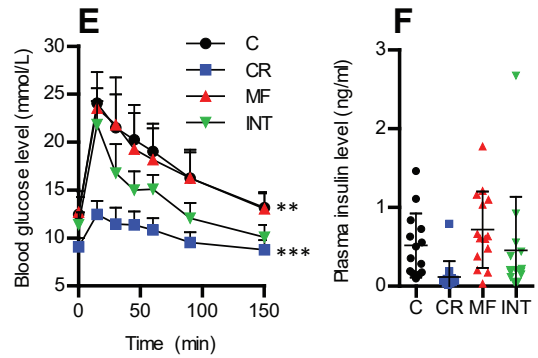

Figure 1. Beneficial effects of an INT diet regimen on body, WAT and liver weight, food intake and glucose tolerance. (A) Weekly measurement of body weight. (B) WAT weight and the ratio to the body weight. (C) Liver weight, ratio to body weight, and liver weight correlation with body and WAT weight. (D) Energy intake measurement at 12 months old of age, daily (left) and weekly (right). (E) Glucose clearance measured by an oral glucose tolerance test. (F) Fasting plasma insulin levels. Error bars reflect standard deviation (SD). $\left.) P\left(0.05 ;{ }^{* *}\right) P<0.01 ;{ }^{* *}\right) P<0.001$ vs the INT group.

\section{Eating behaviour and total energy intake in mice exposed to the intermittent diet}

As anticipated, the switch between high/low food availability strongly affected the eating behaviour of INT-exposed mice. The daily energy intake measurement revealed hyperphagia during the first few days of the ad libitum feeding week (Fig. 1D), compensating the $40 \%$ energy reduction in the restricted feeding week, followed by a gradual decrease in food intake. As a result, the actual total energy intake of INT-fed mice was $87.9 \%$ of the mean intake of the C-fed mice (Supplementary

Table 3A). Furthermore, since the INT-fed mice received the MF diet during the week they were fed ad libitum, overall these mice consumed more fat, $20.5 \%$ of the total energy intake, compared to $9 \%$ in the $\mathrm{C}$ group (Supplementary Table 3B). 


\section{Tolerance to glucose was maintained in mice exposed to intermittent diet}

To establish the effects of the different diets on glucose metabolism, an oral glucose tolerance test (OGTT) was carried out in the mice 2 weeks prior to sacrifice. The maximal glucose level of INT-exposed mice was lower and returned more rapidly to the basal fasting level than that of the $C$ and MF groups ( $P<0.01$, Fig. 1E), though not to the extent as observed in the CR group. Similarly, the area under the curve (AUC) of INT diet group was significantly lower than $C$ and MF groups $(P<0.001$, Supplementary Fig. 1), but not as low as the AUC of CR diet group. Furthermore, averaged fasting plasma insulin level was lower in INT-fed mice compared to mice that received the $C$ and MF diets (Fig. 1F), although this effect did not reach statistical significance $(P>0.05)$ due to the large inter-individual variation between the mice in the different intervention groups.

\section{Intermittent diet protected the liver from developing the biochemical and histological marks of NAFLD}

Next, we evaluated the effects of the dietary interventions on various biochemical and morphological features related to the development of NAFLD. Fatty liver development was examined by quantification of the hepatic triglyceride (TG) content. A very low TG content in mice exposed to the CR and INT diets was observed (Fig. 2A). Mice fed the $\mathrm{C}$ or MF diet showed hepatic TG deposition with a large inter-individual difference, of which most of the animals exceed the diagnosis level of NAFLD, >5-10\% (or 50-100 mg TG/ g liver) [29]. The level of TG content strongly correlated with body weight ( $r=0.83$, Fig. 2B) and liver weight ( $r=0.91$, Supplementary Fig. 2A).

To determine whether hepatic function was affected due to exposure to the different diets, serum levels of alanine aminotransferase (ALT) and aspartate aminotransferase (AST), two well-established markers of liver injury/damage, were measured. Interestingly, the lowest ALT and AST levels were detected in the INT group ( $P<0.05$, Fig. 2 C and 2D). In C- and MF-fed mice we found that the elevated level of the ALT was correlated with increasing hepatic TG levels (Supplementary Fig. 2B).

Histological analysis was carried out to examine whether the different diets induced morphological changes in the liver. Haematoxylin-eosin (H-E) staining of liver sections of $\mathrm{C}$ - and MF-fed mice with high body weights (the highest tertile of bodyweight), of which elevated hepatic TG levels were measured, revealed fat accumulation with development of macro- and microvesicular lipid droplets (Fig. 2E). On the other hand, animals with the low body weights (the lowest tertile of body weight) of the $C$ and MF groups displayed normal liver morphology, similar to animals of the CR and INT intervention groups with both low and high body weight. To assess the progression of hepatic fibrosis, we performed a collagen Sirius-red staining (Fig. 2F). The results obtained showed that the high body weight-animals of the $C$ and MF groups developed collagen scarring, indicating the presence of hepatic fibrosis in these mice, while this effect was not observed in CR and INT-fed mice. 
A

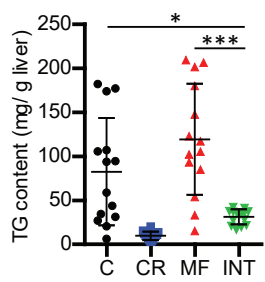

B

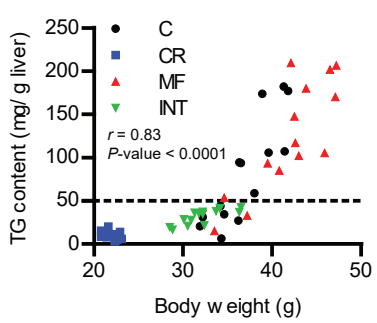

C

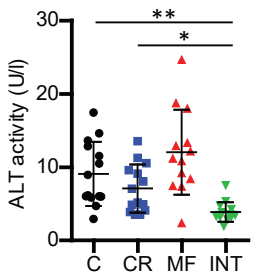

D

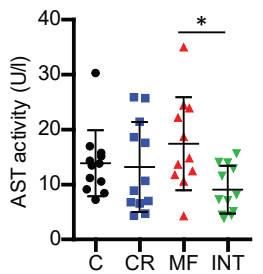

E C

$\mathrm{CR}$

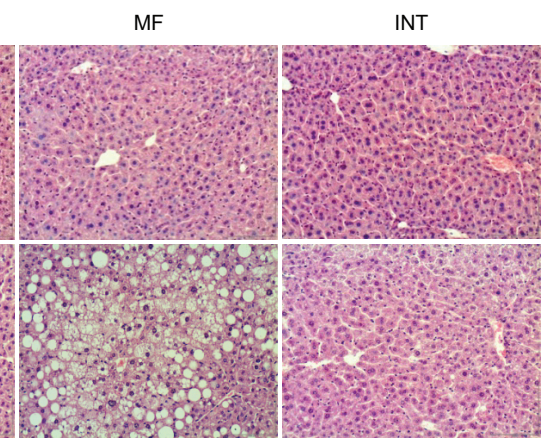

F

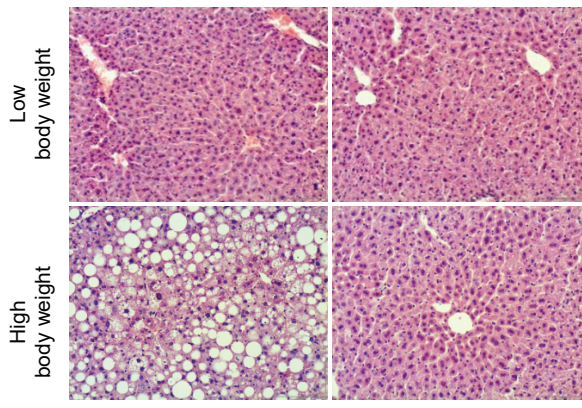

C

CR

MF

INT

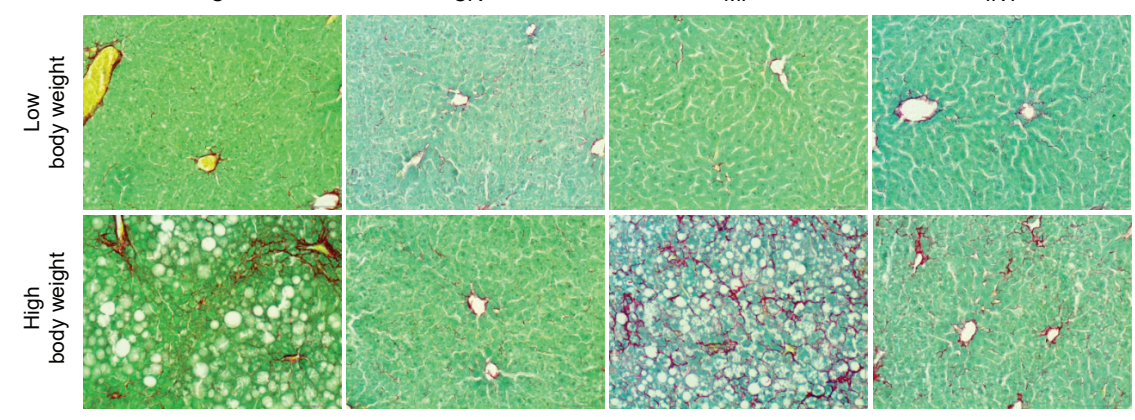

Figure 2. Hepatic TG, plasma ALT and AST and liver histology indicated NAFLD development in C- and MFfed mice, but not in mice exposed to CR and INT. (A) Hepatic TG content. (B) Correlation between hepatic TG and body weight. (C) Plasma ALT. (D) Plasma AST. Error bars reflect SD. *) $P<0.05$; **) $P<0.01$; **) $P<0.001$ vs. INT group. (E) H-E staining and (F) FCF Green and Sirius Red staining of liver section of animals with the high and low body weight (original magnification 200x).

\section{Gene expression profiles reflected the body weight similarity amongst the $C$, MF and INT groups, but not for the CR group}

To obtain an overview of the molecular profiles induced by the different dietary interventions, a microarray analysis was carried out on the livers of the mice of all four intervention groups. An unsupervised clustering analysis was performed and a dendrogram plot of the gene expression profile similarity is depicted in Figure 3A. The clustering analysis was performed on all genes, but due to space limitation the plot is represented by the top 250 most variable genes based 
on interquartile range. The dendrogram is complemented by a heatmap of the body weight, representing each animal. Three major clusters were observed: 1) a cluster of all animals in the CR diet group, 2) a cluster consists of the $\mathrm{C}$ and MF animals that mostly containing a high body weight, and 3) a cluster consists of animals with moderate body weight from the C, MF and mostly INT diet group. The clustering and sub-clustering patterns of the second and third clusters suggested that the gene expression profile of the C, MF and INT diet groups is strongly correlated with body weight similarity. Interestingly, the gene expression profile of the CR-exposed mice was distinct from those of the mice exposed to the INT diet, despite the fact that the physiological, biochemical and morphological features of the mice in the CR and INT diet groups, e.g. hepatic TG content, were similar. 


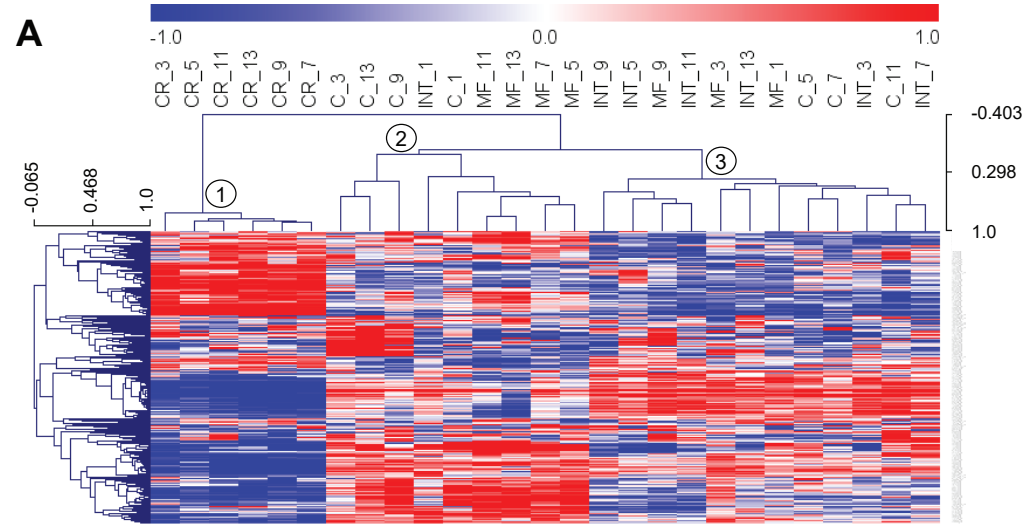

Body weight

B

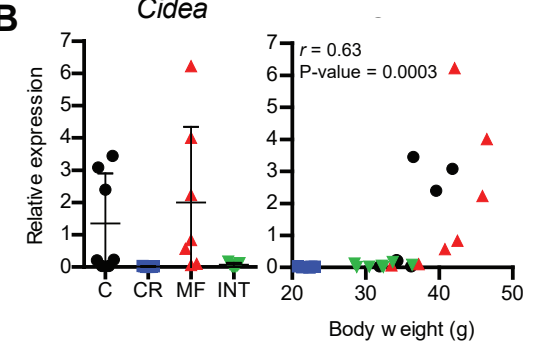

C

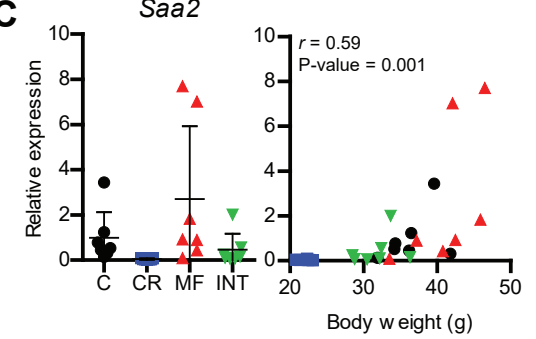

D

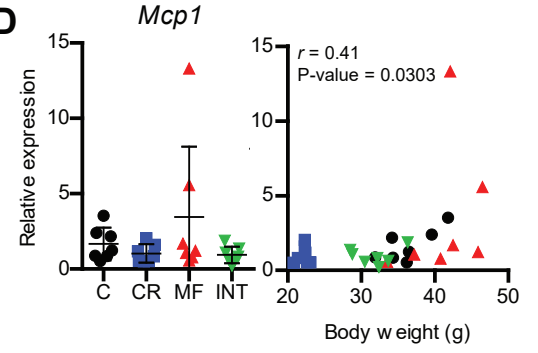

Cidec

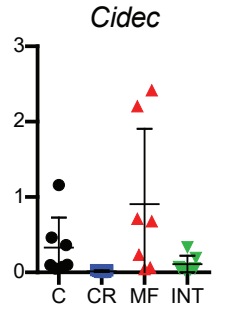

Len2
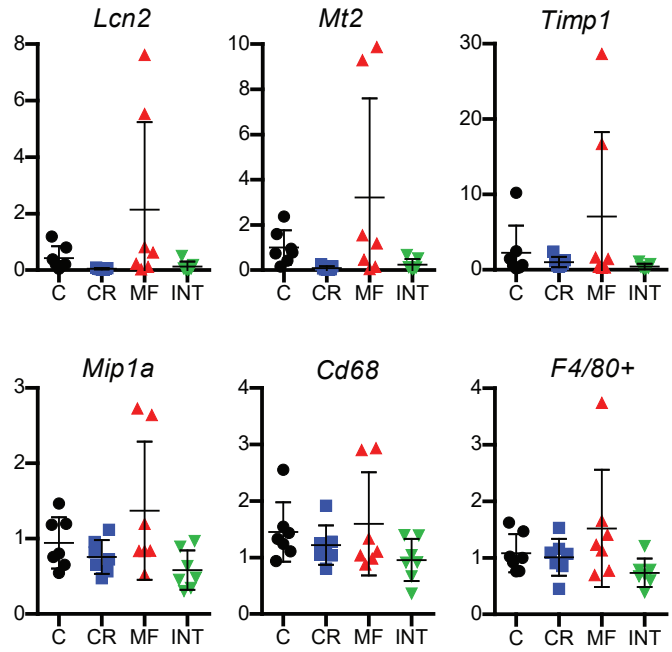

Figure 3. INT-fed mice displayed a similar gene expression profile to $C$ - and MF-exposed mice with similar body weights. (A) A hierarchical clustering plot depicting the liver gene expression profiles similarity of the different diet regimens. The color band under the plot represents the body weight of individual mice in a white-to-red color scale (white = low values, red $=$ high values). Q-PCR analysis on all animals of all dietary intervention groups shows differential expression of genes involved in (B) lipid droplet formation, (C) inflammatory and fibrosis, and (D) macrophage/monocyte recruitment. No statistical difference was found for gene expressions in INT-fed mice compared to the other intervention groups. Error bars represent SD. 


\section{Expression of genes associated with lipid droplet formation were increased with increasing body weight}

To examine NAFLD development in INT-fed animals at a molecular level in more detail, we analysed the expression of genes involved in lipid droplet formation, which are known to be highly correlated with the severity of NAFLD [30-32]. Q-PCR analysis was performed on all mice of all four intervention groups for cell death-inducing DFFA-like effector A (Cidea) and C (Cidec), monoacylglycerol 0-acyltransferase 1 (Mogat1) and fibroblast growth factor 21 (Fgf21) (Fig. 3B). The expression level of microarray and Q-PCR analysis showed to correspond to each other.

Mean expressions levels of the INT-fed mice for all four genes were lower than that of the $C$ and MF groups. However, the observed differences did not reach statistical significance, most likely due to the large inter-individual difference in expression levels observed in the C-and MF-fed mice, which was similar to the observed physiological features. Comparison of gene expression and body weight revealed significant positive correlations ( $r=0.63-0.74)$ for all four genes (Fig. 3B and Supplementary Fig. 3A). These results suggest that increased body weight might promote lipid droplet formation leading to NAFLD, while the INT regimen maintained a moderate body weight and low expression of genes involved in lipid droplet formation.

\section{High body weight is essential but not sufficient for NASH pathogenesis}

The effects of different diets on hepatic inflammation and fibrosis were assessed by Q-PCR analysis of four genes: serum amyloid A 2 (Saa2), lipocalin 2 (Lcn2), metallothionein (Mt2) and tissue inhibitor of metalloproteinase 1 (Timp1). The results presented in Fig. $\mathbf{3 C}$ revealed low expression levels of all four genes in all diet groups, except for a few animals in the $C$ and MF groups which consistently showed elevated expression levels. Plotting of the gene expression levels versus body weight revealed that elevated expression levels of the inflammatory/fibrosisrelated genes were exhibited by the $\mathrm{C}$ - and MF-exposed animals with the highest body weight (Fig. 3C and Supplementary Fig. 3B), in which the positive correlations were considered significant ( $P$ $<0.001-0.0102)$. However, not all animals with high body weight showed elevated gene expression of the markers for inflammation and fibrosis, which was indicated by a weaker body weight-gene expression correlation compared with the lipid droplet formation genes $(r=0.48-0.59)$.

In addition, expression levels of previously established macrophage/monocyte marker genes linked to NASH pathogenesis [28], including monocyte chemoattractant protein 1 (Mcp1), macrophage inflammatory protein la (Mipla), cell surface glycoprotein $\mathrm{F} 4 / 80\left(\mathrm{~F} 4 / 80^{+}\right)$and CD68 antigen (Cd68), were analysed. Expression levels of these macrophage/monocyte markers were generally low in all diet groups and an elevated level was only observed for a few animals in the 
MF group (Fig. 3D and Supplementary Fig. 3C). It should be noted that elevated expression of inflammation and fibrosis genes was consistently found in the same animals having the highest body weight, explaining the weak correlations between the expression levels of monocyte/macrophage markers gene and body weight $(r=0.39-0.49)$.

Taken together, these results indicate that hepatic inflammation and macrophage/ monocyte infiltration occur in only a few animals with high body weight mainly in the MF group. However, since not all mice having a high body weight showed these elevated gene expression levels of these markers, body weight is not necessarily an indicator of hepatic inflammation or infiltration.

\section{DISCUSSION}

In this study, we showed that a weekly alternating diet consisting of $40 \mathrm{E} \% \mathrm{CR}$ and ad libitum feeding of a MF (25E\%) diet prevented development of NAFLD/NASH, which was induced by lifelong exposure to a MF diet and even the low-fat control diet. INT-fed mice maintained glucose tolerant, showed normal insulin levels and low plasma ALT and AST levels. Furthermore, they did not exhibit signs of hepatic steatosis and fibrosis, indicated by the hepatic triglyceride levels and morphology observations. Interestingly, all metabolic parameters measured were improved in INT-fed mice and were found to be highly similar to the results obtained from mice that have been continuously exposed to a CR diet. Likewise, the INT and CR intervention groups exhibited no sign of hepatic lipid accumulation, inflammation or macrophage/monocyte infiltration at the gene expression level.

Studies examining the effects of an increased dietary fat content on NAFLD development typically apply a high percentage of fat (40-60E\%) to resemble the Western-style diet [33]. Hepatic steatosis is then acutely induced and developed at young age (3-4 months) [33]. However, the prevalence of NAFLD has been shown to increase with advancing age and this indicates that the adverse effect of Western diet accumulates over many years [34-37]. Therefore, in this study we applied a long-term exposure to a less extreme diet by using a fat content of $25 \mathrm{E} \%$, which we expect to simulate a slow onset of NAFLD developed at middle-age time point by the consumption of MF diet. We demonstrated that the long-term exposure of male C57BL/6 J mice to a MF diet until the age of 12 months resulted in pronounced weight gain, impaired glucose clearance, elevated insulin levels and an increase in plasma ALT levels. Hepatic steatosis and fibrosis were observed in some but not all mice which had the highest body weight. Together our results reveal that 1 ) 
long-term exposure to a MF diet seriously impairs metabolic homeostasis and is a risk factor for NAFLD development, and 2) applying every-other-week 40E\% CR largely reversed the adverse health effects induced by the MF diet in 12-month-old mice.

Health-promoting effects of a CR diet have been commonly recognized, but compliance to such a diet is challenging for most individuals [15], and therefore an INT diet is explored as the alternative. Our results indicated that the adverse health effects of a MF diet could be reversed by applying every-other-week CR, however, it should be noticed that most metabolic parameters measured showed slightly better outcomes in the continuous CR-fed mice compared to the INT group. This result suggests that the INT regimen does not fully bestow all beneficial metabolic improvements of the CR diet. Only plasma ALT revealed significantly lower levels in INT-fed mice compared to mice that have received the CR diet. ALT is a transaminase enzyme and it has previously been shown that CR induces hepatic gluconeogenesis, accompanied by increased transamination activity as the first step in amino acid catabolism, resulting in elevated plasma ALT level [38]. In addition, it should be noted that, although the outcomes of the INT diet regimen are novel as it discovers a remarkable improvement on liver parameters compared to a MF diet, we cannot exclude that the health benefits observed are (partially) due to lower body weights, in addition to the alternating of the diets.

We found that, despite the similarity in energy intake between $C$ and MF diet group, the animals that received a higher fat content in their diet developed a higher body weight. This has been observed in more studies $[25,39]$ and possibly results from the lower energy requirement to store excess energy from fat than from carbohydrate, due to the additional conversion of glucose to fatty acids. However, our results demonstrate that body weight gain and not the type of the diet, is a stronger predictor of hepatic steatosis development, e.g. liver mass, hepatic TG accumulation and expression of genes associated with lipid droplet formation. Then, the development hepatic fibrosis and inflammation seemed to be triggered by additional factor(s) in addition to body weight, as not all animals with the highest body weight developed fibrosis or inflammation, supporting the role of the "second hit" required in the development of NASH as first proposed by Day and James [40].

Substantial inter-individual variation in all analysed features was observed between mice of the $\mathrm{C}$ and MF groups. Heterogeneity in the response to diets is a previously reported characteristic of C57BL/6J mice [41, 42]. We previously also showed that, upon exposure to a low-fat or highfat diet, low or high responders could be distinguished in both intervention groups. The low and high responders revealed differences in food intake, body-weight gain, adiposity, a broad range of plasma markers, and liver features [30]. A number of possible mechanisms behind the observed variation in C57BL/6J mice have been proposed, such as copy number variations in the mouse 
genome and epigenetic features, but the exact underlying cause is not known yet [42, 43].

The results obtained in this study showed that in all four intervention groups both WAT and liver weight correlate, as expected, with body weight. However, the ratio of WAT- and liver-to-body weight in INT-exposed mice revealed an intriguing weight distribution. While relative WAT weight was higher than that of CR-fed mice, relative liver weight was significantly lower, suggesting a potential beneficial health effect for the liver of the INT compared to the CR diet. When more energy is consumed than needed for daily energy expenditure, the excess is in first instance stored in WAT, which has the capacity to expand in order to store the surplus of lipids and to prevent ectopic fat deposition. However, there is a limit to the lipidstorage capacity of WAT and when this is exceeded, spill-over is suggested to occur resulting in fat storage in organs, such as heart, pancreas, skeletal muscle and liver [44]. When fat accumulation in the liver exceeds the threshold of 50-100 mg TG/ g liver, this results in liver steatosis (Fig. 4A). Thus, the fact that the relative WAT weight was much higher than the relative liver weight in INT-fed mice suggests that energy excess consumed during the ad libitum MF week is primarily stored in WAT, but the energy deficiency during the CR week is compensated by releasing fat from the liver, instead of fat utilisation from WAT (as illustrated in

Fig. 4B). This hypothesis is supported by a previous finding showing a rapid liver fat clearance (up to $29.6 \%$ decrease of liver TG content) occurring after 48 hours of CR in humans [11]. Although the exact mechanism of the rapid liver fat extraction is unknown, this is an intriguing feature of the INT regimen that might contribute notably to NAFLD prevention by the INT diet.

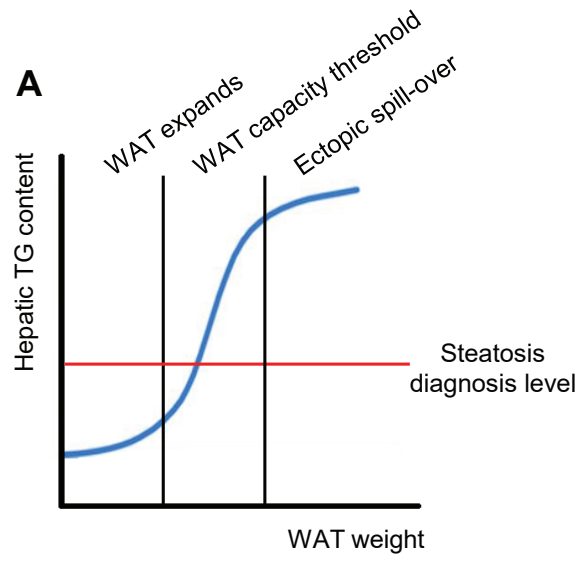

B

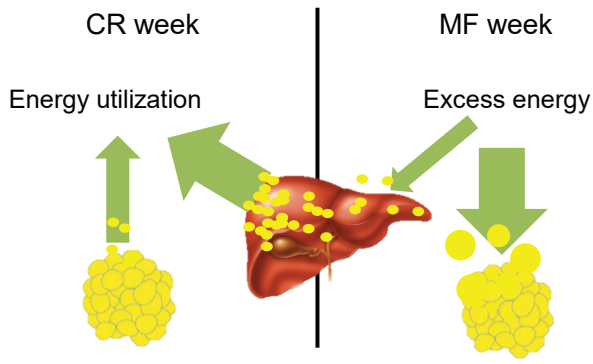

Figure 4. Proposed models for how the fat storage is distributed in WAT and liver. (A) Excess fat is initially stored in WAT, which may expand until a certain threshold. If the lipid-storage capacity is reached, ectopic fat deposition starts to occur in various organs, such as liver. (B) During the ad libitum feeding week, the excess energy is mainly stored in WAT, but the compensation of deficit energy during the restricted feeding week might be mobilized predominantly from the liver fat storage. This proposed mechanism of an alternating diet may prevent hepatic steatosis development. 
Remarkably, our results revealed that, while the metabolic profile of INT-fed mice was comparable to that induced by the CR diet, the INT- and CR-fed mice showed different transcriptomic profiles. As the INT-fed mice were sacrificed during their ad libitum MF feeding week, gene expressions patterns observed were most likely regulated to adapt to the ad libitum feeding condition to maintain homeostasis. This suggests that the transcriptome is a more flexible phenotype, and more closely resembles the ad libitum-fed groups. Unfortunately, due to the limited number of animals in this study, we were not able to assess the liver transcriptomics of INT mice during their restricted feeding week.

In conclusion, the results obtained in this study show that a weekly alternating INT diet varying between 40E\% CR and ad libitum MF feeding prevents NAFLD development. Mice exposed to the INT diet retain healthy physiological features as displayed by continuous exposure to $C R$, while the gene expression profile was shown to be dissimilar with CR, but more similar to that of $\mathrm{C}$ - and MFfed mice with comparable body weight. 


\section{REFERENCES}

1. Lim, S., Shin, H., Song, J.H., Kwak, S.H., et al., Increasing Prevalence of Metabolic Syndrome in Korea: The Korean National Health and Nutrition Examination Survey for 1998-2007. Diabetes Care, 2011, 34,13231328.

2. Riediger, N.D. and Clara, I., Prevalence of metabolic syndrome in the Canadian adult population. Can. Med. Assoc. J., 2011, 183,E1127-E1134.

3. Shen, J., Goyal, A., and Sperling, L., The Emerging Epidemic of Obesity, Diabetes, and the Metabolic Syndrome in China. Cardiology Research and Practice, 2012, 2012,5.

4. Beltrán-Sánchez, H., Harhay, M.O., Harhay, M.M., and McElligott, S., Prevalence and Trends of Metabolic Syndrome in the Adult U.S. Population, 1999-2010. J. Am. Coll. Cardiol., 2013, 62,697-703.

5. Blachier, M., Leleu, H., Peck-Radosavljevic, M., Valla, D.-C., et al., The burden of liver disease in Europe: A review of available epidemiological data. J. Hepatol., 2013, 58,593-608.

6. Bellentani, S., Scaglioni, F., Marino, M., and Bedogni, G., Epidemiology of Non-Alcoholic Fatty Liver Disease. Dig. Dis., 2010, 28,155-161.

7. de Wit, N.J.W., Afman, L.A., Mensink, M., and Müller, M., Phenotyping the effect of diet on non-alcoholic fatty liver disease. J. Hepatol., 2012, 57,1370-1373.

8. Spindler, S.R., Caloric restriction: From soup to nuts. Ageing Research Reviews, 2010, 9,324-353.

9. Fontana, L., Partridge, L., and Longo, V.D., Extending Healthy Life Span-From Yeast to Humans. Science, 2010, 328,321-326.

10. Chung, K.W., Kim, D.H., Park, M.H., Choi, Y.J., et al., Recent advances in calorie restriction research on aging. Exp. Gerontol., 2013, 48,1049-1053.

11. Kirk, E., Reeds, D.N., Finck, B.N., Mayurranjan, M.S., et al., Dietary Fat and Carbohydrates Differentially Alter Insulin Sensitivity During Caloric Restriction. Gastroenterology, 2009, 136,1552-1560.

12. Yu, H., Jia, W., and Guo, Z., Reducing Liver Fat by Low Carbohydrate Caloric Restriction Targets Hepatic Glucose Production in Non-Diabetic Obese Adults with Non-Alcoholic Fatty Liver Disease. Journal of Clinical Medicine, 2014, 3,1050-1063.

13. Cava, E. and Fontana, L., Will calorie restriction work in humans? Aging (Albany NY), 2013, 5,507-514.

14. Barnosky, A.R., Hoddy, K.K., Unterman, T.G., and Varady, K.A., Intermittent fasting vs daily calorie restriction for type 2 diabetes prevention: a review of human findings. Translational Research, 2014, 164,302-311.

15. Racette, S.B., Weiss, E.P., Villareal, D.T., Arif, H., et al., One year of caloric restriction in humans: Feasibility and effects on body composition and abdominal adipose tissue. Journals of Gerontology - Series A Biological Sciences and Medical Sciences, 2006, 61,943-950.

16. Goodrick, C.L., Ingram, D.K., Reynolds, M.A., Freeman, J.R., et al., Effects of intermittent feeding upon body weight and lifespan in inbred mice: interaction of genotype and age. Mech. Ageing Dev., 1990, 55,6987.

17. Anson, R.M., Guo, Z., de Cabo, R., lyun, T., et al., Intermittent fasting dissociates beneficial effects of dietary restriction on glucose metabolism and neuronal resistance to injury from calorie intake. Proceedings of the National Academy of Sciences, 2003, 100,6216-6220.

18. Tikoo, K., Tripathi, D.N., Kabra, D.G., Sharma, V., et al., Intermittent fasting prevents the progression of type I diabetic nephropathy in rats and changes the expression of Sir2 and p53. FEBS Lett., 2007, 581,10711078.

19. Beigy, M., Vakili, S., Berijani, S., Aminizade, M., et al., Alternate-day fasting diet improves fructose-induced 
insulin resistance in mice. J. Anim. Physiol. Anim. Nutr., 2013, 97,1125-1131.

20. Reeves, P.G., Nielsen, F.H., and Fahey, G.C., AIN-93 Purified Diets for Laboratory Rodents: Final Report of the American Institute of Nutrition Ad Hoc Writing Committee on the Reformulation of the AIN-76A Rodent Diet. The Journal of Nutrition, 1993, 123,1939-1951.

21. Bolstad, B.M., Irizarry, R.A., Åstrand, M., and Speed, T.P., A comparison of normalization methods for high density oligonucleotide array data based on variance and bias. Bioinformatics, 2003, 19,185-193.

22. Irizarry, R.A., Bolstad, B.M., Collin, F., Cope, L.M., et al., Summaries of Affymetrix GeneChip probe level data. Nucleic Acids Res., 2003, 31 ,e15.

23. Dai, M., Wang, P., Boyd, A.D., Kostov, G., et al., Evolving gene/transcript definitions significantly alter the interpretation of GeneChip data. Nucleic Acids Res., 2005, 33,e175.

24. Saeed, A.I., Sharov, V., White, J., Li, J., et al., TM4: a free, open-source system for microarray data management and analysis. BioTechniques, 2003, 34,374-378.

25. Steegenga, W., de Wit, N., Boekschoten, M., IJssennagger, N., et al., Structural, functional and molecular analysis of the effects of aging in the small intestine and colon of C57BL/6J mice. BMC Medical Genomics, $2012,5,38$.

26. Wang, X. and Seed, B., A PCR primer bank for quantitative gene expression analysis. Nucleic Acids Res., 2003, $31, \mathrm{e} 154$.

27. Misener, S., Krawetz, S., Rozen, S., and Skaletsky, H., Primer3 on the WWW for General Users and for Biologist Programmers, in Bioinformatics Methods and Protocols. 1999, Humana Press: New Jersey. p. 365-386.

28. Stienstra, R., Mandard, S., Patsouris, D., Maass, C., et al., Peroxisome Proliferator-Activated Receptor a Protects against Obesity-Induced Hepatic Inflammation. Endocrinology, 2007, 148,2753-2763.

29. Vanni, E., Bugianesi, E., Kotronen, A., De Minicis, S., et al., From the metabolic syndrome to NAFLD or vice versa? Digestive and Liver Disease, 2010, 42,320-330.

30. Duval, C., Thissen, U., Keshtkar, S., Accart, B., et al., Adipose Tissue Dysfunction Signals Progression of Hepatic Steatosis Towards Nonalcoholic Steatohepatitis in C57Bl/6 Mice. Diabetes, 2010, 59,3181-3191.

31. Zhou, L., Xu, L., Ye, J., Li, D., et al., Cidea promotes hepatic steatosis by sensing dietary fatty acids. Hepatology, 2012, 56,95-107.

32. Li, H., Fang, Q., Gao, F., Fan, J., et al., Fibroblast growth factor 21 levels are increased in nonalcoholic fatty liver disease patients and are correlated with hepatic triglyceride. J. Hepatol., 2010, 53,934-940.

33. Buettner, R., Schölmerich, J., and Bollheimer, L.C., High-fat Diets: Modeling the Metabolic Disorders of Human Obesity in Rodents. Obesity, 2007, 15,798-808.

34. Mozumdar, A. and Liguori, G., Persistent Increase of Prevalence of Metabolic Syndrome Among U.S. Adults: NHANES III to NHANES 1999-2006. Diabetes Care, 2011, 34,216-219.

35. Frith, J., Day, C.P., Henderson, E., Burt, A.D., et al., Non-Alcoholic Fatty Liver Disease in Older People. Gerontology, 2009, 55,607-613.

36. Amarapurkar, D., Kamani, P., Patel, N., Gupte, P., et al., Prevalence of non-alcoholic fatty liver disease: population based study. Ann Hepatol, 2007, 6,161-3.

37. Lee, J.Y., Kim, K.M., Lee, S.G., Yu, E., et al., Prevalence and risk factors of non-alcoholic fatty liver disease in potential living liver donors in Korea: a review of 589 consecutive liver biopsies in a single center. J. Hepatol., 2007, 47,239-244.

38. Hagopian, K., Ramsey, J.J., and Weindruch, R., Caloric restriction increases gluconeogenic and transaminase enzyme activities in mouse liver. Exp. Gerontol., 2003, 38,267-278.

39. de Wit, N., Bosch-Vermeulen, H., de Groot, P., Hooiveld, G., et al., The role of the small intestine in the development of dietary fat-induced obesity and insulin resistance in C57BL/6J mice. BMC Medical Genomics, 2008, 1,14. 
40. Day, C.P. and James, O.F.W., Steatohepatitis: A tale of two "hits"? Gastroenterology, 1998, 114,842-845.

41. Burcelin, R., Crivelli, V., Dacosta, A., Roy-Tirelli, A., et al., Heterogeneous metabolic adaptation of C57BL/6J mice to high-fat diet. American Journal of Physiology - Endocrinology and Metabolism, 2002, 282,E834-E842.

42. Koza, R.A., Nikonova, L., Hogan, J., Rim, J.-S., et al., Changes in Gene Expression Foreshadow DietInduced Obesity in Genetically Identical Mice. PLoS Genet., 2006, 2,e81.

43. Cutler, G. and Kassner, P.D., Copy number variation in the mouse genome: implications for the mouse as a model organism for human disease. Cytogenet. Genome Res., 2008, 123,297-306.

44. Unger, R.H., Clark, G.O., Scherer, P.E., and Orci, L., Lipid homeostasis, lipotoxicity and the metabolic syndrome. Biochimica et Biophysica Acta (BBA) - Molecular and Cell Biology of Lipids, 2010, 1801,209-214.

\section{AUTHOR CONTRIBUTIONS}

F.R. designed the research, collected data, analysed the data, wrote, reviewed and edited the manuscript. M.V.B. analysed the data and reviewed and edited the manuscript. A.A.Z. and C.L. collected and analysed the data. M.M. designed the research and reviewed and edited the manuscript. W.T.S. designed the research, collected data, analysed the data, and reviewed and edited the manuscript.

No potential conflicts of interest relevant to this article were reported.

\section{ACKNOWLEDGEMENTS}

This work was financially supported by European Union's Seventh Framework Programme (FP7/2007-2011) IDEAL-aging under grant agreement no. 259679. 


\section{SUPPLEMENTARY TABLES}

Supplementary Table 1. Composition of the experimental diet. The CR diet was adjusted for the vitamins and minerals amount to ensure a homologous intake between both groups.

\begin{tabular}{l|ccc}
\multicolumn{1}{c}{ AIN-93W } & AIN-93W-CR & AIN-93W-MF \\
\hline Energy (kcal/g) & 3.85 & 3.77 & 4.25 \\
Energy from fat (\%) & 9 & 10 & 25 \\
Energy from protein (\%) & 15 & 15 & 13 \\
Energy from carbohydrates (\%) & 76 & 75 & 61 \\
Mineral mix AIN-93M (g\%) & 35 & 50 & 35 \\
Vitamin mix AIN-93M (g\%) & 10 & 14 & 10 \\
Choline bitartrate (g\%) & 2.5 & 3.5 & 2.5
\end{tabular}

Supplementary Table 2. List of primer sequence used in Q-PCR analysis

\begin{tabular}{|c|c|c|}
\hline Gene name & Forward primer $\left(5^{\prime} \rightarrow 3^{\prime}\right)$ & Reverse primer $\left(5^{\prime} \rightarrow 3^{\prime}\right)$ \\
\hline Cidea & TGACATTCATGGGATTGCAGAC & GGCCAGTTGTGATGACTAAGAC \\
\hline Cidec & ATGGACTACGCCATGAAGTCT & CGGTGCTAACACGACAGGG \\
\hline Mogat1 & TCCCGTTGTTCCGAGAATATCT & TGCTCAGCACATGAGACAAAC \\
\hline Fgf21 & GTGTCAAAGCCTCTAGGTTTCTT & GGTACACATTGTAACCGTCCTC \\
\hline Saa2 & GCGAGCCTACACTGACATGA & TाTTCTCAGCAGCCCAGACT \\
\hline Lcn2 & TGGAAGAACCAAGGAGCTGT & GGTGGGGACAGAGAAGATGA \\
\hline Mt2 & GCCTGCAAATGCAAACAATGC & AGCTGCACTTGTCGGAAGC \\
\hline Timp1 & GCAACTCGGACCTGGTCATAA & CGGCCCGTGATGAGAAACT \\
\hline Mcpl & CCCAATGAGTAGGCTGGAGA & TCTGGACCCATTCCTTCTTG \\
\hline Mip1a & СCTCTGTCACCTGCTCAACA & GTAGACTCACATGGCGCTGA \\
\hline $\mathrm{F} 4 / 80^{+}$ & CTTTGGCTATGGGCTTCCAGTC & GCAAGGAGGACAGAGTTATCGTG \\
\hline Cd68 & CCAATTCAGGGTGGAAGAAA & CTCGGGCTCTGATGTAGGTC \\
\hline $18 \mathrm{~s}$ & CGGCTACCACATCCAAGGA & ССАATTACAGGGCCTCGAAA \\
\hline
\end{tabular}


Supplementary Table 3A. Relative energy intake of different diets

\begin{tabular}{l|cccc} 
& Control & CR & MF & INT \\
\hline $\begin{array}{l}\text { Energy intake } \\
\text { (kcal/week) }\end{array}$ & $103.2 \pm 9.4$ & $60.8 \pm 1.1$ & $116.9 \pm 12.0$ & $90.7 \pm 8.1$ \\
$\begin{array}{l}\text { Relative energy } \\
\text { intake (\%) }\end{array}$ & 100 & 58.9 & 113.3 & 87.9 \\
& & & &
\end{tabular}

Supplementary Table 3B. Nutrient intake of the different diets

\begin{tabular}{ll|cccc}
\multicolumn{2}{c}{ Nutrients } & Control & CR & MF & INT \\
\hline Carbohydrate & Kcal & $78.6 \pm 7.7$ & $45.6 \pm 0.8$ & $67.4 \pm 9.0$ & $59.3 \pm 4.9$ \\
& $\%$ & 76.0 & 75.0 & 61.6 & 65.9 \\
Protein & Kcal & $15.5 \pm 1.5$ & $9.1 \pm 0.2$ & $14.4 \pm 1.9$ & $12.3 \pm 1.0$ \\
& $\%$ & 15.0 & 15.0 & 13.1 & 13.7 \\
Fat & Kcal & $9.3 \pm 0.9$ & $6.1 \pm 0.1$ & $27.6 \pm 3.7$ & $18.4 \pm 2.0$ \\
& $\%$ & 9.0 & 10.0 & 25.3 & 20.5
\end{tabular}




\section{SUPPLEMENTARY FIGURES}

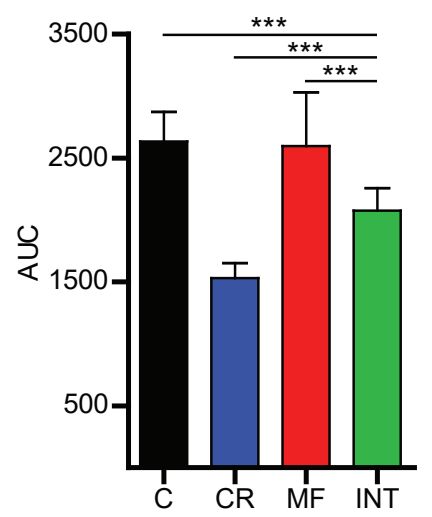

Supplementary Figure 1. The area under the curve (AUC) of oral glucose tolerance test (OGTT) of INT diet group significantly lower than the $\mathrm{C}$ and MF diet groups, but not to the extent of CR diet group.
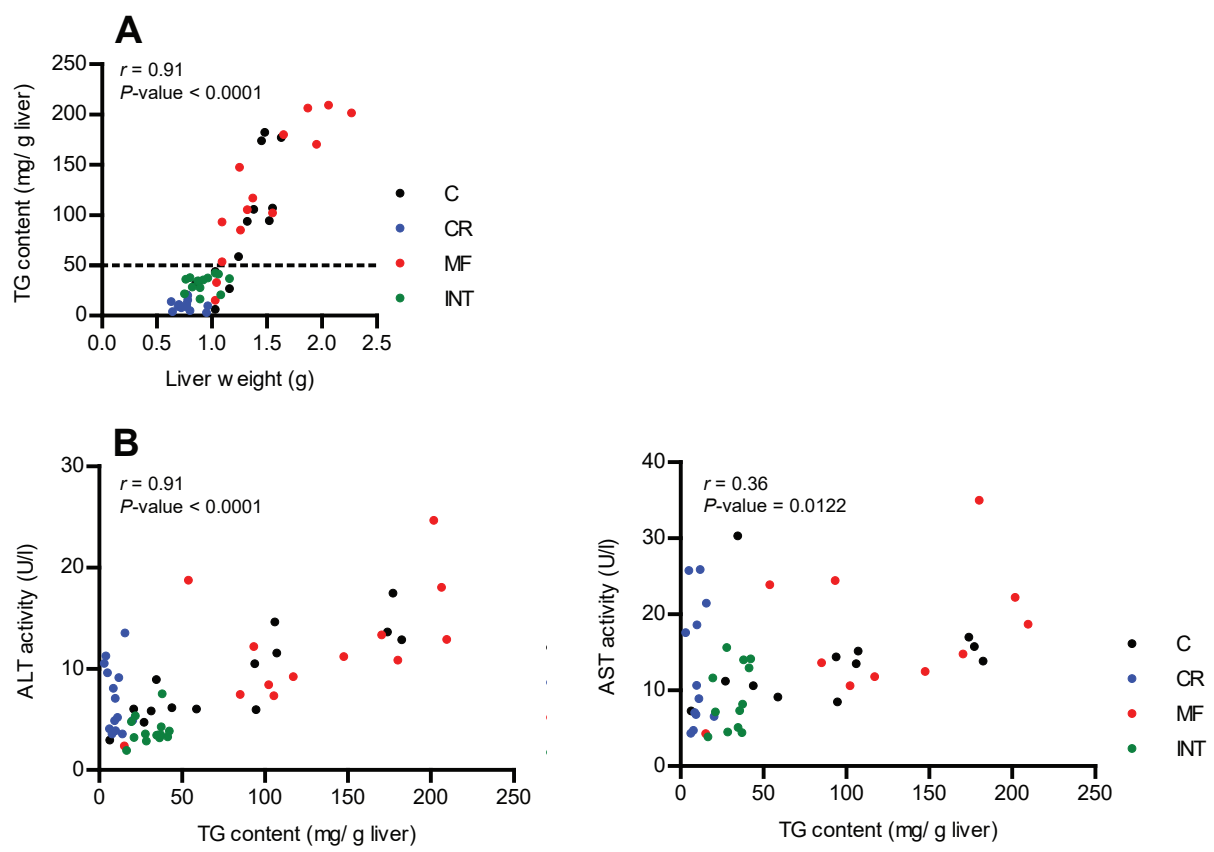

Supplementary Figure 2. (A) A correlation between liver TG content and liver weight. (B) A correlation between plasma ALT and AST level to the liver TG content 
A

Cidec

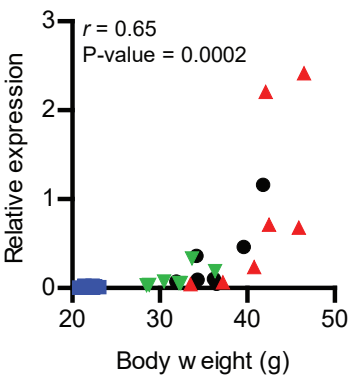

B

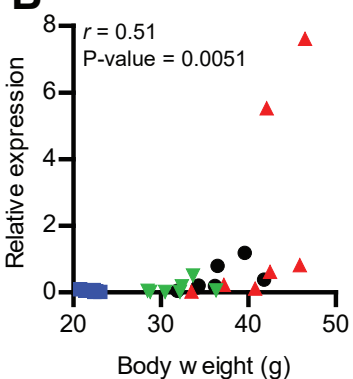

C Mip1a

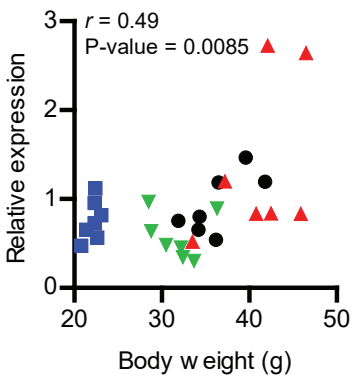

Mogat1

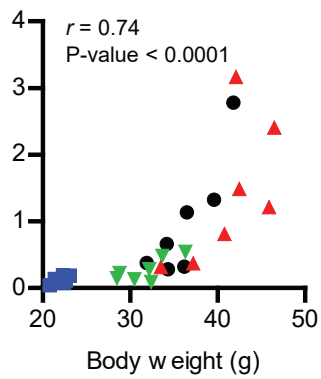

Mt2

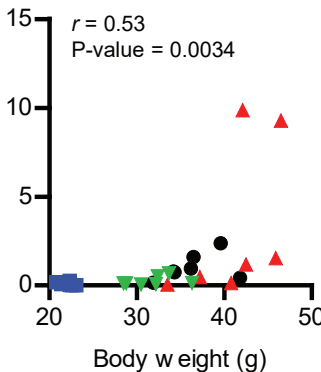

Cd68

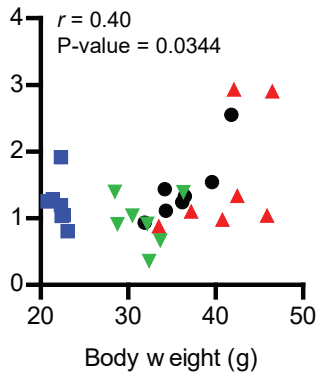

Fgf21

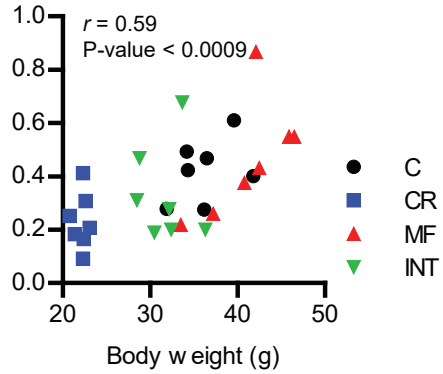

Timp1

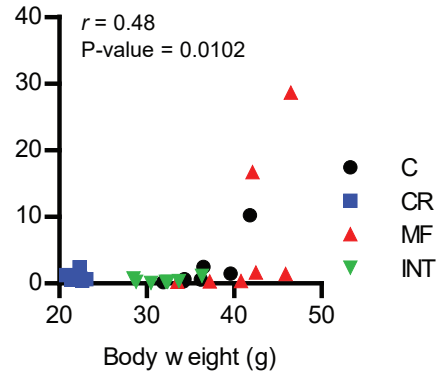

F4/80+

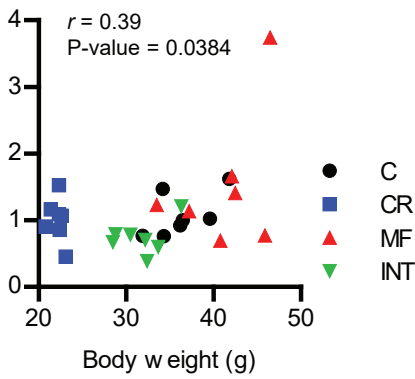

Supplementary Figure 3. A correlation between body weight and expression of genes involved in (A) lipid droplet formation, (B) hepatic inflammation and fibrosis genes, (C) macrophage/monocyte infiltration genes. 



\section{ABSTRACT}

Calorie restriction (CR), without malnutrition, has been shown to extend life and health span in model species. For most humans, a life-long CR diet is too arduous to adhere to and poses adverse side effects. The aim of this study was to explore whether weekly intermittent CR can reverse the adverse short- and long-term effects of a medium-fat (MF) diet in aging mice. For this purpose we have exposed C57BL/6J mice to an intermittent (INT) diet, alternating weekly between $\mathrm{CR}$ and ad libitum feeding of a MF diet. This weekly intermittent CR significantly counteracted the adverse effects of the MF diet on mortality, body weight and a wide range of liver health markers in 24-month-old mice. Transcriptome analysis of the liver revealed that hepatic gene expression profiles of INT-exposed animals were much more comparable to CR than to MF-exposed mice. At 12 months of age, a subgroup of MF-exposed mice was transferred to the INT diet. Of the 2815 genes displaying differential expression between the INT- and MF-exposed mice, 1510 genes changed to the INT-expression profile during the 12 months after the diet switch. However, a small subset of 148 genes were identified that were consistently changed by the MF diet during the first phase of life. Our results suggest that MF-induced deregulated PXR activity persistently affects lipid and xenobiotic metabolism in the liver of the old diet switch mice. In conclusion, weekly intermittent CR largely, but not completely, reversed the short- and long-term effects caused by a MF diet. 


\section{ABSTRACT}

The beneficial health effects of a calorie restricted (CR) diet, avoiding malnutrition, can be appreciated from two perspectives. First, in our obesogenic society where about half of the population is overweight or obese [1], maintaining a reduced energy intake is the best nutritional strategy to achieve and maintain weight loss [2, 3]. Since obesity causes a wide range of serious chronic diseases, the negative energy balance induced by a CR diet will result in weight loss and persuade, concomitantly, health promoting effects. Secondly, CR has been acknowledged as the most successful approach to increase longevity in a wide range of species [4]. Apart from life-span, reduced food intake also increases health-span. Here, the health-promoting effect is not achieved by counteracting obesity-related disorders but by ameliorating a wide range of aging-related diseases $[5,6]$. It is worth mentioning, however, that these obesity- and age related disorders fundamentally overlap.

In order to achieve longevity, the application of a CR diet requires life-long adherence to a very strict dietary regimen. Severe food restriction is very arduous and too difficult to practice and to sustain for most individuals. Importantly, long-term exposure to a CR diet might also cause substantial side effects like amenorrhoea, osteoporosis, decreased fertility and libido, impaired wound healing and increased susceptibility to infections [7-10]. Health-promoting effects have been reported by different variants of the CR diet, practising intermittent energy restriction [11-13]. By applying repetitive cycles of fasting/CR and eating, the negative side effects of $\mathrm{CR}$ are thought to be circumvented. Moreover, an intermittent CR regimen is more feasible to hold on to. Importantly, increasing evidence points out that the beneficial health effects of (intermittent) CR are not solely caused by reduced body weight $[14,15]$. Timing and limitation of meal frequency affect the circadian rhythm and might induce a repetitive challenge that most likely will contribute to the health promoting effects [16-18].

One of most frequently affected organs in obese individuals is the liver, which is recognized to be the most important metabolic organ and supporting nearly every other organ in the body. Obesity induces a spectrum of abnormalities in the liver called non-alcoholic fatty liver disease (NAFLD), which is currently the most common chronic liver disease in developed countries. NAFLD is seen in $20-40 \%$ of the general adult population, but incidence in severe obese adult individuals is much higher (70-90\%) [19]. The mildest form of NAFLD is simple hepatic steatosis (HS) and is characterized by intrahepatic accumulation of lipids alone. In around $47 \%$ of the affected individuals this benign hepatic lipid accumulations evolves into non-alcoholic steatohepatitis (NASH) characterized by inflammatory infiltration of the liver and low-level fibrosis [20]. Between 10 to 29\% 
of the individuals with NASH develop advanced fibrosis, cirrhosis and ultimately, hepatocellular carcinoma (HCC) [21, 22]. This progressive disease development is characterized by increasing severity and predisposition to mortality. It has been reported that both the prevalence of NAFLD as well as progression into more severe forms of NAFLD in the general population increase with age [23-25]. The early stages of NAFLD are considered to feature a benign, non-progressive and reversible disease [19]. Management of HS and NASH is mainly focused on treatment of obesity by introducing lifestyle modifications including increased exercise and decreased calorie intake. Although there is strong evidence that the early stages of NAFLD caused by an obesogenic diet are reversible, it is currently not clear whether all detrimental effects are completely restored after long-term exposure to such a diet.

In this study we explored the effects of intermittent calorie restriction (INT) regimen on NAFLD development during aging by applying a relatively robust form of the INT diet in male C57BL/6 J mice. By alternating weekly between a $40 \mathrm{E} \%$ calorie restricted control diet and an ad libitum medium-fat (MF) diet, the mice were challenged to adapt to differences in 1) energy intake, 2) macronutrient composition of the diet and 3) constant food exposure versus an one-portion-a-day feeding pattern. The effects of this challenging diet on overall body health were explored. In addition, we examined the biochemical, morphological and molecular effects this diet caused in the liver of 24-month-old mice. The obesity-counteracting effects of this diet were identified by introducing a diet switch in life-long MF exposed mice at the age of 12 months to the INT diet. In 24-month-old mice we examined into what extent the adverse health effects caused by the MF diet are reversible on whole-body as well as on liver health. Life-long exposure to a low-fat control diet (C), MF or a continuous CR diet (30\% calorie reduction) were included as normal, unhealthy and healthy aging controls, respectively. 


\section{RESULTS}

\section{Intermittent calorie restriction protected against the detrimental effects of a medium-fat diet}

To explore the effects of weekly intermittent CR a panel of different physiological markers were measured. Body weight was recorded weekly during the study and showed, as expected, that the MF-exposed mice gained the highest body weight while the lowest body weight was observed for the CR-fed animals (Figure 1A). Bodyweight of the INT-fed mice displayed a constantly fluctuating pattern, dependent on the diet the mice received in the preceding week. DEXA scan analysis showed that, in line with the bodyweight, the percentages of fat ( $f$ ) and lean (l) body mass of the INT-exposed mice (l: 68.3\%, f: 31.7\%) were in between those of the CR- (l: 78.9\%, f: $20.1 \%$ ) and C-fed mice (l: 64.4\%, f: 35.6\%) (Figure 1B). Food intake recordings presented in Figure 1C show that, during the entire study, of all intervention groups the INT-exposed mice have the highest energy intake during the ad libitum feeding week and the lowest during their CR-restricted week. Combining the intake values of the CR and ad libitum MF weeks at 24 month of age revealed that the mean energy intake of the INTfed mice was slightly lower than the amount of calories consumed by $\mathrm{C}$-fed mice and substantially higher than that consumed by the CR-exposed mice (Figure 1D). Furthermore, it is important to note that this figure also reveals that, although the INT-exposed mice consume less kcal/week than the control mice, their fat intake is higher. Consequently, INT-exposed mice were challenged by fluctuating amount of calories, but also by a difference in macronutrient composition of the consumed diet. We have recently shown that 12-month-old mice exposed to the INT diet demonstrate hyperphagia during the first few days of the ad libitum feeding week [26]. This increased eating pattern at the first days after the diet switch still occurred at old age (Supplemental Figure S1A).

Daily activity recorded at the age of 23 months revealed that the INT-exposed animals were significantly more active than the C- and MF-exposed mice and similarly active to the life-long CRexposed animals (Figure 1E). Although this increased activity pattern in the INT-exposed mice was found in both the CR and the ad libitum MF feeding week, the timing of the activity differed (see Supplemental Figure S1B). During the MF-week the burst of activity was observed at the moment that the light was switched off. During the CR-week the activity increased when the food was provided, 30 minutes before the light was switched off similar to the pattern found for the life-long CR exposed mice. Mortality rates of the 4 intervention groups, presented in Figure 1F, show the highest survival in CR-fed animals. The survival rate of this intervention group was significantly higher than that of the $C$-fed $(p=0.001)$ and MF-fed $(p<0.001)$ mice. The survival rate of the INT-exposed mice was lower than that of the CR-fed mice, but still significantly enhanced $(p=0.04)$ compared to the MF-exposed mice. 

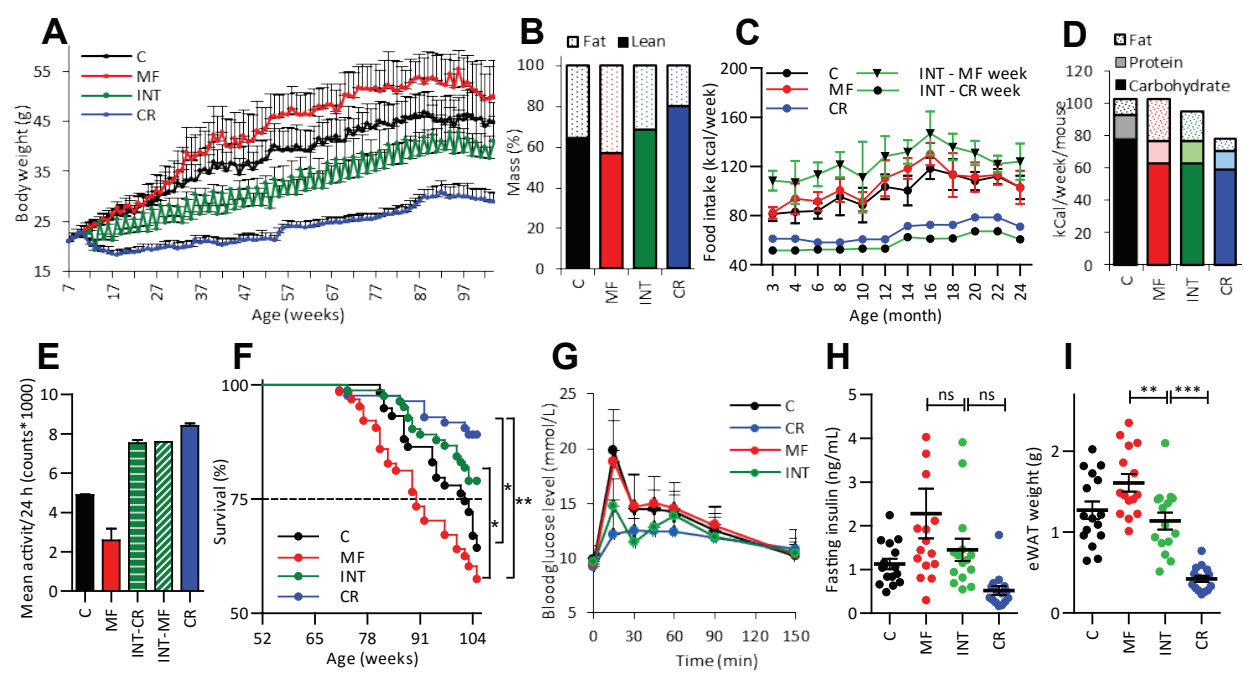

Figure 1. Intermittent CR strongly increases life span and health span. (A) Weekly bodyweight measurements show a fluctuating body weight of the INT-exposed mice dependent on the diet the mice received in the preceding week and a mean body weight in between that of the C- and CR-exposed animals. (B) INT-exposed mice display an increase in lean body mass but not to the same extent as found in the CR-fed animals. (C) During the whole study the highest food intake was found for the ad libitum fed mice from the INT group while the during the CR week this intervention group had the lowest food intake. (D) Mean energy intake of the INT-fed mice is slightly lower compared to the MF-exposed animals and significantly higher compared to the CR-fed mice. (E) Mean daily activity levels in the INT-fed mice were highly similar to the CR-exposed animals and differ significantly from the MF-exposed mice. (F) Weekly intermittent CR causes a significant increase in survival compared to the MF-exposed mice but not to the same extent as found for the CR-fed animals. (G) An OGT test showed that glucose clearance in the INT-exposed mice is similar to CR-fed mice. (H) Fasting insulin levels of the INF-fed mice were in between the levels found in the MF and CR-exposed mice. (I) eWAT weight of the INT-exposed animals was significantly lower compared to the MF-fed mice but significantly higher than found for the CR-exposed mice.

An oral glucose tolerance test (OGTT), carried out 2 weeks prior to sacrifice, revealed that glucose clearance in the INT-fed mice was strongly improved compared to the MF-exposed mice and almost similar to the CR-fed mice (Figure 16). In addition, fasting insulin levels in the INTexposed mice showed a tendency to decrease compared to the MF-exposed mice, although this effect was not significant (Figure 1H). No significant changes between the intervention groups was found for plasma free fatty acid (FFA), triglyceride (TG), plasminogen activator inhibitor 1 (PAI-1) and resistin (Supplemental Figure S1C). Weight of the epididymal white adipose tissue (eWAT) in the INT-exposed mice was found to be significantly decreased compared to the MF-exposed mice and significantly increased compared to the CR intervention group (Figure 1I). A similar effect was found for kidney weight but not for heart, lung, spleen and pancreas weight (Supplemental Figure S1D).

Taken together, intermittent CR significantly improved the overall health compared to the MFfed mice, indicating a protection against the detrimental effects of a MF diet. However, the beneficial effects were not as pronounced as induced by the continuous CR exposure. 


\section{Life-long weekly intermittent calorie restriction reduced most but not all detrimental effects caused by a MF diet in the liver}

To evaluate the effects of life-long INT feeding on the liver, a panel of different markers were examined in 24-month-old mice. Liver weight in the INT-fed mice was significantly lower compared to the MF-exposed mice and did not differ significantly from the CR-fed mice (Figure 2A). A significant decrease in the intrahepatic triglyceride (IHTG) levels was found in the INT-fed mice compared to the MF-exposed mice, but the levels were significantly higher compared to the CR-exposed mice (Figure 2B). Histological analysis of the livers of the mice from the different intervention groups confirmed the results of the IHTG measurements (Supplemental Figure S2A and B). Moreover, intermittent CR caused significant improvement of plasma alanine aminotransferase (ALT) levels, a well-established marker of liver injury/damage compared to the mice exposed to the MF diet (Figure 2C). In addition, histological scoring of inflammatory aggregates (Figure 2D) and quantification of liver fibrosis by measuring 4-hydroxyproline levels (Figure 2E) showed significant improvement of liver health of the INT-fed mice compared to the MF-exposed mice. These last three markers represent the more advanced stages of NAFLD and revealed no significant differences between the INT- and CR-exposed mice.

In summary, despite the fact that INT-fed animals had been exposed to the MF diet for half of their life, apart from displaying slight accumulation of liver triglycerides, they performed equally well as CR-exposed mice for all other NAFLD markers tested.
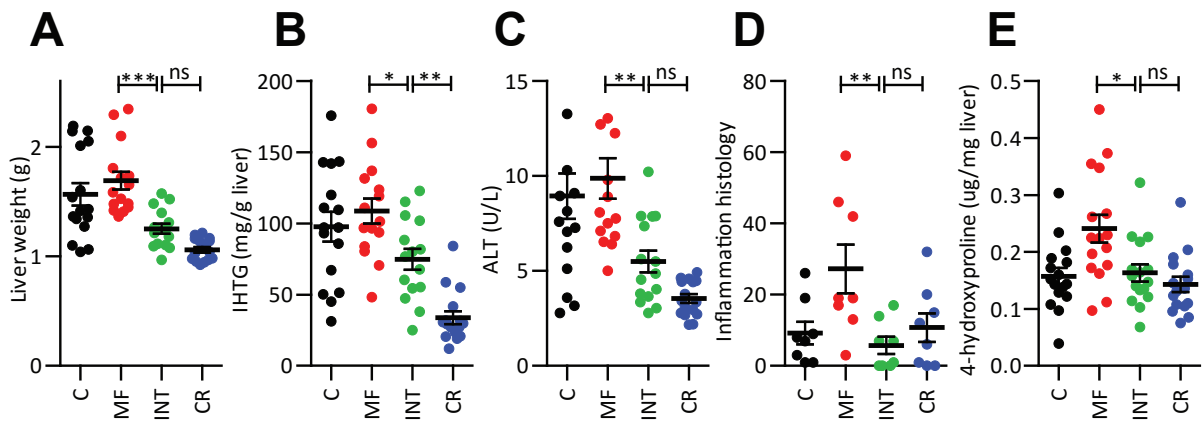

Figure 2. Intermittent CR counteracts the adverse health effects of the MF diet on the liver. The adverse effects caused by a MF diet on (A) liver weight, (B) IHTG, (C) ALT, (D) inflammation histology and (E) 4-hydroxyproline levels were significantly counteracted by intermittent $\mathrm{CR}$. 


\section{Gene expression profiles of 24-month-old INT-fed mice were more similar to CR than to MF-exposed animals}

Next, we studied the differences in hepatic gene expression patterns of the INT-exposed mice in comparison to the CR and MF intervention groups. For this purpose, microarray (MA) analysis was applied on RNA isolated of the livers from the mice. As shown in Figure $\mathbf{3 A}$, the number of significantly $(p<0.01)$ differentially expressed genes between INT- and CR-exposed mice was most abundant in 6-month-old mice and decreased during aging. In contrast, the difference between INTand MF-exposed animals increased with age and the highest number of differentially expressed genes was found at the oldest time point. At the age of 24 months, in total 569 genes were found to be differential expressed between INT- and CR-fed mice while 2815 genes displayed differential expression between INT- and MF-exposed animals. Principal Component Analysis (PCA) of the top-1000 most variable genes in the 24-month-old animals, showed higher similarity between the INT and the CR-fed animals than between the INT- and MF-exposed mice (Figure 3B). Ingenuity pathway analysis (IPA) revealed that the differentially expressed genes in the two comparisons are related to different "Diseases and disorders". Genes related to "Neurological disease, cancer and hereditary disorders" were found to be differentially expressed between INT- and CR-exposed mice (Table 1). Differential gene expression between INT- and MF-exposed mice was found to include genes involved in "Immunological, endocrine and gastrointestinal diseases" (representing mostly diabetes-related genes) (Table 1). In addition, in the top canonical pathways RXR activation was the major difference between INT- and CR-fed mice while immune pathway related genes dominate the difference between INT- and MF-exposed animals. Furthermore, IPA revealed that for hepatoxicityrelated processes, differential gene expression between INT and CR diet groups pointed towards differences in fat and bilirubin metabolism while differential gene expression between INT and MFexposed mice were involved in the advanced stages of NAFLD-related processes including fibrosis and cirrhosis (Table 1). This result confirms the biochemical data presented in Figure 2.

In conclusion, these results indicate that in 24-month-old mice, gene expression profiles obtained from the liver of INT-exposed mice are distinct from the MF-fed mice and resemble more the molecular features of CR-exposed animals. This result indicates that weekly intermittent CR evidently alters gene expression profiles induced by the MF diet at old age. 


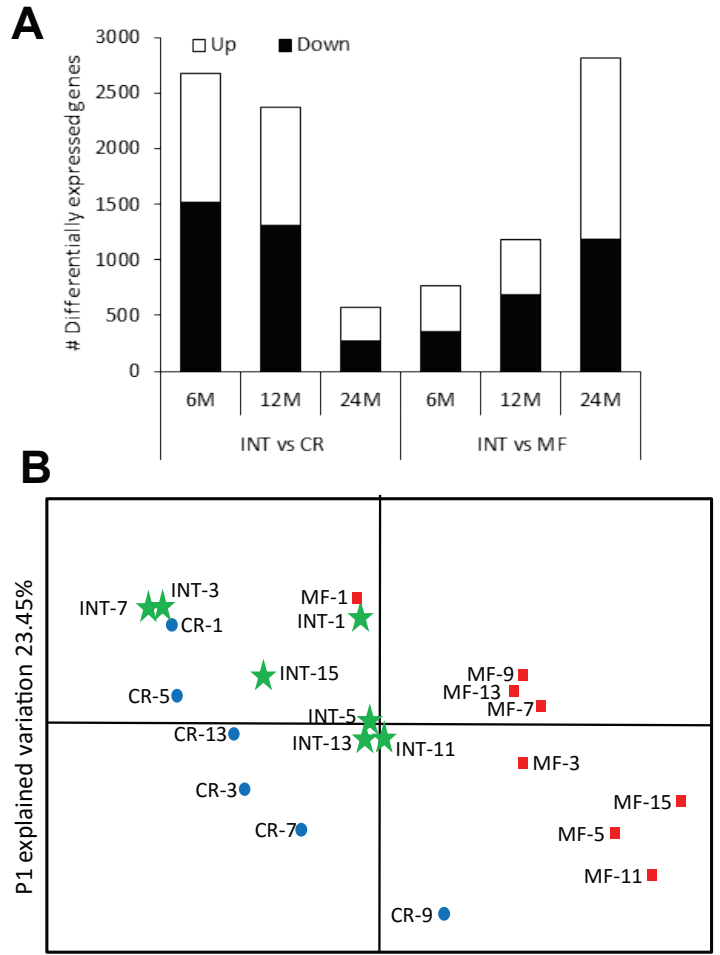

Figure 3. Gene expression in the liver of 24-months-old INT-exposed mice is more similar to that of CR than MF-fed animals. (A) The number of genes significantly differentially expression between INT and CR-exposed mice decreases during aging while this number increase during aging between the INT and MF-exposed intervention groups. (B) The PCA plot generated from the top-1000 most variable genes revealed that the expression profiles of the INT-fed animals is more similar to the CR than to the MF-exposed animals.

Table 1. Functional differences in the differentially regulated genes

24M INT vs CR

24M INT vs MF

\begin{tabular}{|c|c|c|c|c|}
\hline \multicolumn{2}{|r|}{ Category } & \multicolumn{2}{|c|}{ p-value $\quad$ Category } & \multirow{2}{*}{$\begin{array}{l}\text { p-value } \\
2.66 \mathrm{E}-41-5.26 \mathrm{E}-9\end{array}$} \\
\hline Diseases & Neurological disease & $2.81 \mathrm{E}-5-2.54 \mathrm{E}-2$ & Immunological disease & \\
\hline \multirow[t]{2}{*}{$\begin{array}{l}\text { and } \\
\text { disorders }\end{array}$} & Cancer & $3.30 \mathrm{E}-5-2.54 \mathrm{E}-2$ & $\begin{array}{l}\text { Endocrine systems } \\
\text { disorders }\end{array}$ & $6.01 \mathrm{E}-39-5.60 \mathrm{E}-29$ \\
\hline & Hereditary disorders & $3.92 E-5-2.54 E-2$ & Gastrointestinal disease & $6.01 E-39-7.43 E-9$ \\
\hline \multirow{3}{*}{$\begin{array}{l}\text { Top } \\
\text { canonical } \\
\text { pathways }\end{array}$} & $\begin{array}{l}\text { LPS/IL-1 mediated inh. } \\
\text { of RXR function }\end{array}$ & 8.87E-07 & $\begin{array}{l}\text { iCOS-iCOSL signalling } T \\
\text { helper cells }\end{array}$ & $3.78 \mathrm{E}-12$ \\
\hline & PCR/RXR activation & 7.00E-06 & $\begin{array}{l}\text { Role of NFAT in immune } \\
\text { response reg. }\end{array}$ & 4.43E-12 \\
\hline & TR/RXR activation & 5.87E-05 & IL-8 signalling & 8.47E-12 \\
\hline \multirow{3}{*}{$\begin{array}{l}\text { Hepa- } \\
\text { toxicity }\end{array}$} & Liver cholestasis & $8.50 \mathrm{E}-5-1.86 \mathrm{E}-2$ & Liver cirrhosis & $5.97 \mathrm{E}-10-2.35 \mathrm{E}-1$ \\
\hline & Liver steatosis & $2.14 \mathrm{E}-4-1.43 \mathrm{E}-1$ & Liver hyperplasia & $1.63 E-9-6.09 E-1$ \\
\hline & Liver hyperbilirubinemia & $1.18 \mathrm{E}-3-5.02 \mathrm{E}-2$ & Liver fibrosis & $5.23 E-7-4.86 E-1$ \\
\hline
\end{tabular}




\section{Phenotypic plasticity observed in the MF/INT diet switch group}

In the final part of our analysis we explored the plasticity of the long-term effects induced by the MF diet. For this purpose, a subset of 12-month-old mice was transferred from the MF to the INT diet (MF/INT diet switch group). The mice of the diet switch group received the INT diet till sacrifice at the age of 24 months. As shown in Figure 4A, the results of the OGTT analysis revealed that glucose metabolism of the mice in the diet switch group was similar to that of the life-long INT-exposed mice. Furthermore, survival of the diet switch group markedly increased compared to the life-long MF-exposed animals, although this effect did not reach the level of significance (Figure 4B). Compared with the life-long MF-exposed mice body weight decreased significantly in the diet switch mice (Figure 4C). A reduction in eWAT (Figure 4D) and liver (Figure 4E) weight was observed but both adaptations were not significant. Analysis of a panel of liver health markers (Figure 4F) revealed no change in IHTG levels after the diet switch and a marked but not significant decrease in plasma ALT, lymphocyte infiltration and 4-hydroxyproline levels (Figure 4G-I) in the MF/INT diet switch mice compared to the life-long MF-exposed animals. 

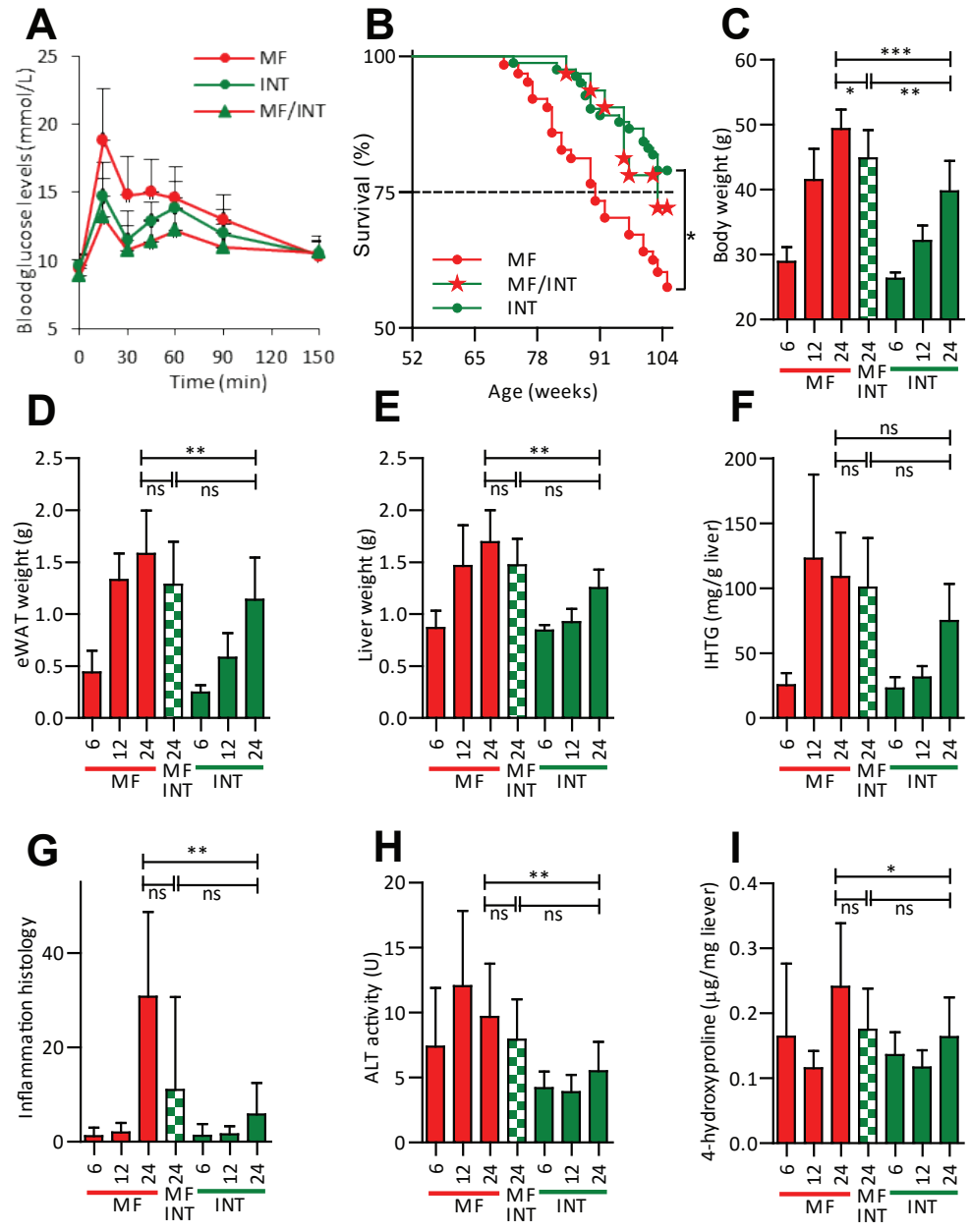

Figure 4. Effects of exposure to a MF diet are partially reversed after transferring the mice to the INT diet for the last 12 months of their life. Strong adaptation to the INT diet in the MF/INT diet switch group was found by measuring (A) glucose clearance (B) survival and (C) body weight. The decrease in (D) eWAT weight, (E) liver weight, (F) IHTG levels, (G) liver inflammatory aggregates, (H) plasma ALT and (I) 4-hydroxyproline levels after the diet switch were not significant.

\section{Molecular adaptations and irreversible changes in the liver of the MF/INT diet switch group}

As shown in Figure 3A, microarray analysis revealed that at the age of 24 months 2815 genes displayed significant differentially expression between INT- and MF-exposed mice. We compared the expression levels of these $\mathbf{2 8 1 5}$ genes between the diet switch group with either the life-long MFor INT-exposed animals, respectively (for an overview see Supplemental Figure S3A). Expression levels of 1510 genes were found to be similar in the MF/INT and the life-long INT-exposed animals 
and distinct from the MF-exposed animals (MF/INT vs INT $p>0.01$; MF/INT vs MF $p<0.01$ ). This result indicated that expression levels of these 1510 genes adapted to the INT diet the mice had received during the last 12 months of their life. Expression levels of a second subset of 1157 genes did not differ significantly from either the MF or from the INT-fed animals (MF/INT vs INT $p>0.01$; MF/INT vs MF $p>0.01$ ). Interestingly, expression of a relative small subset of 148 genes differed significantly between the MF/INT diet switch and the INT-exposed mice, but not from the MF-exposed mice (MF/ INT vs INT $p<0.01$; MF/INT vs MF $p>0.01$ ). This result implies that the differential expression induced by the MF diet of these 148 genes did not adjust to the INT diet during the last 12 months of life, thus indicating an irreversibility of the MF-induced effects. The heatmap of the hierarchical clustering of this selection of 148 genes presented in Figure 5A shows clustering of the INT-exposed mice and a distinct expression profile compared to mice of the MF and MF/INT intervention groups. IPA applied to compare the canonical pathways of the 148 irreversible (Supplemental Table S1) and the 1510 reversible genes, revealed that these subsets of genes represented distinct canonical pathways. The 148 consistently altered genes were found to affect RXR-mediated processes and xenobiotic metabolism signalling (Figure 5B). In contrast, the 1510 adaptive genes were found to be involved in a variety of immune response and inflammation-related pathways and in hepatic fibrosis/stellate cell activation (Figure 5C). IPA was applied to obtain insight into the mechanisms regulating the expression of the 148 consistently altered genes and found that PXR was the strongest regulator of this selection of genes (Supplemental Table S2).

In Figure 5D expression profiles of $H s d 3 b 5, C d 36$ and Ppary are shown, representing 3 examples of genes of the subset of 148 consistently altered genes (other examples are presented in Supplemental Figure S3B). Interestingly, for most of the genes displaying consistently changes induced by the MF diet, significantly different expression between the INT and MF-exposed mice starts at a young age ( 6 or 12 months). Figure 5E presents the expression levels of Cyp2u1, Lpl and Clec10a, showing that the mean expression levels in the MF/INT-exposed animals differs strongly from the life-long MF-exposed mice. For these genes of which the expression levels adapted to the INT diet, no marked changes were found between young MF- and INT-exposed animals (other examples are presented in Supplemental Figure S3C). By analysing the age-related effect of the 148 consistently altered genes in more detail we found that $43 \%$ of these genes showed a MFinduced change in gene expression at young age ( 6 or 12 months) while this was found for only $9 \%$ of the 1510 adaptive genes.

In conclusion, differential expression regulation of irreversible genes might have an onset earlier in life than the genes of which the expression levels adapt to the INT diet. 


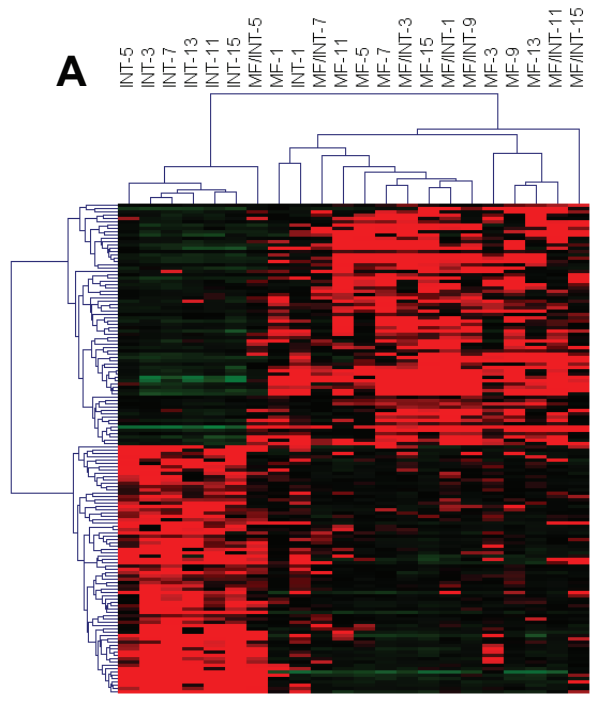

B Top canonical pathways 148 selection LPS/IL-1 Med. Inhibition of RXR Function Xenobiotic Metabolism Signaling Nicotine Degradation II PXR/RXR Activation

Glutathione-mediated Detoxification FXR/RXR Activation

Aryl Hydrocarbon Receptor Signaling Mineralocorticoid Biosynthesis Glucocorticoid Biosynthesis Hepatic Cholestasis

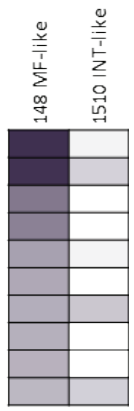

C Top canonical pathways 1510 selection Leukocyte Extravasation Signaling iCOS-iCOSLSignaling in T Helper Cells CD28 Signaling in T Helper Cells Fcil Rec.-med. Phagocytosis in Macr. and Mon. PKCî, Signaling in TLymphocytes Role of NFAT in Regulation of the Imm. Resp. IL-8 Signaling Natural Killer Cell Signaling T Cell Receptor Signaling Dendritic Cell Maturation
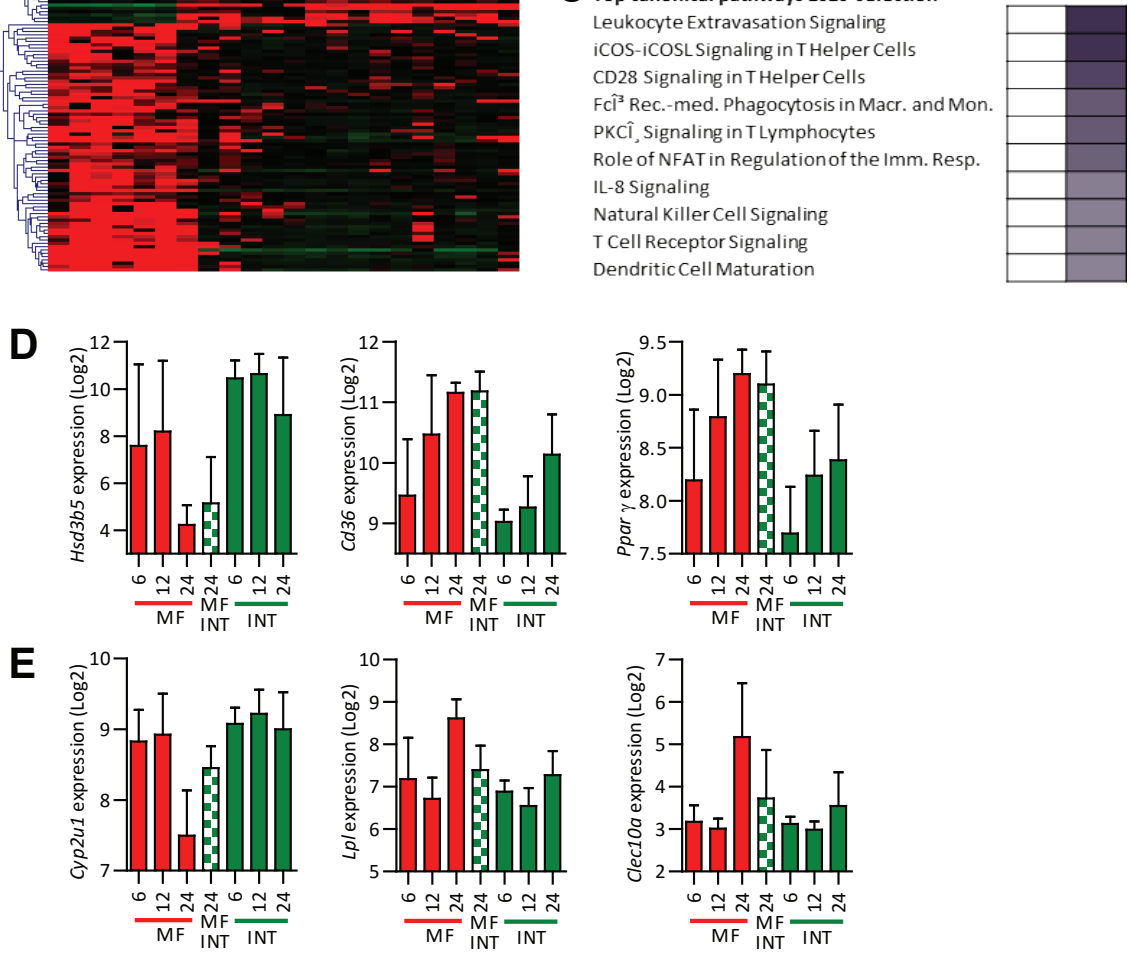

Figure 5. A small fraction of the MF-induced genes during the first $\mathbf{1 2}$ months of life are consistently altered. (A) A heatmap of the 148 consistently altered genes show a different expression profile between the INT compared to the MF and MF/INT diet switch mice. IPA analysis of the (B) 148 consistently changed genes and the (C) 1510 adaptable genes reveal that they present different functional categories. (D) MA profiles of 3 examples of irreversible genes and (E) genes that adapt to the INT diet after the diet switch. 


\section{DISCUSSION}

In this study we examined the effects of life-long intermittent CR in aging mice. We applied a relatively robust variant of this dietary intervention and exposed the mice thereby to multiple challenges. This concept was inspired by the hypothesis that life and health-span extending effects are not necessarily derived from the energy reduction only, but that intermittent exposure to challenges might have an additional health-promoting effect [16-18]. During the experiment the INT-fed mice weekly lose or gain $~ 15 \%$ of their bodyweight, illustrating the constant adaptation of their body to the alternating energy availability. Furthermore, since the composition of the diet also differed between the weeks, the gastrointestinal tract as well as metabolic organs, e.g. the liver, were challenged to handle variations in carbohydrate and fat content. The third challenge was the food exposure time. During the CR week one portion of food was offered 30 minutes prior to the initiation of the dark-phase and the mice consumed the whole portion (almost all) at once. In contrast, during the MF-week the mice had continuous access to food. The results we present show that, although the mean energy intake of the INT-exposed over a two week time interval mice was only marginally (7\%) reduced compared to the MF-exposed mice, the 24-month-old mice display 1) a significant decrease in body weight, 2) a better fat/lean body mass ratio, 3) an improved glucose metabolism and 4) an increased survival. However, it should be noted that, apart from glucose metabolism, life-long CR-exposed mice performed better on all features compared to the INT intervention group.

It is important to take into consideration that, in addition to energy intake reduction and exposure to various challenges, the daily activity of the INT-exposed mice appeared to differ significantly from the MF-exposed mice. We have previously reported increased daily activity in life-long CR-exposed mice in this cohort [27] in line with what has been reported earlier [6]. Since exercise is an important factor regulating health, this feature might very likely contribute to the health improving effects induced by the INT and CR diet. Intriguingly, no difference was found in the daily activity of the INT-exposed mice between the energy restricted and ad libitum feeding week. This suggests that the increased activity can be seen as a habit more than a response to the lack of availability of food [27].

The effects of the INT diet were analysed in more detail in the liver, a central organ in the regulation of metabolic health. NAFLD frequently occurs in obese individuals and is recognized as the hepatic manifestation of metabolic syndrome. In the 24-month-old MF-exposed mice, increased IHTG, liver inflammation, liver 4-hydroxyproline levels and increased plasma ALT levels suggest the presence of advanced stages of NAFLD. Life-long exposure to the CR diet fully protected against 
NAFLD development. INT-fed mice display normal plasma ALT levels, no liver inflammation and no liver fibrosis. These mice, however, display mild steatosis with IHTG levels significantly lower than the MF-exposed mice. This result differs from what we previously have observed in 12-month-old mice where the INT diet improved liver health even beyond the effect achieved by the CR diet [26]. The results we present here indicate that, at the long run, an INT diet protects the liver for the advanced stages of NAFLD. However, this INT diet does not fully prevent MF-induced lipid accumulation. Gene expression profiles generated from the liver of the 24-month-old INT-exposed mice revealed that gene expression in these animals appeared to be more similar to the CR than to the MF-exposed mice, underscoring the beneficial effects of the INT diet on metabolic health. Functional analysis of the differentially expressed genes further confirmed that intermittent CR affects pathways involved in liver fibrosis and cirrhosis and the advanced stages of NAFLD. Taken together, strong improvement in total body and liver health was caused by regular short term exposure to a CR diet. The INT diet almost completely counteracts the effects of the MF diet, which these mice consumed for half of their life. However, despite of the additional challenges these mice have been exposed to the effects did not reach the standards achieved by a life-long CR diet.

CR interventions are often applied to achieve weight loss in overweight and obese subjects. In the second part of our study we explored into what extent, the molecular effects induced by a MF diet in the liver during the first 12 months of life, can be reversed by exposure to the INT diet during the second 12 months of life. Microarray analysis showed significant differential expression of 2815 genes between 24-month-old MF and INT intervention groups. Analysis of the gene expression levels in the MF/INT diet switch group revealed that the majority of these 2815 differentially expressed genes partially ( 1157 genes) or fully (1510 genes) adopted to the INT expression profile. IPA analysis revealed that the subset of 1510 reversible genes represent predominantly inflammation pathways. Similarly, a decrease in plasma ALT levels and liver lymphocyte aggregates and fibrosis was observed in the diet switch mice, although these effects were not significant. Expression levels of a relative small subset of genes (148), however, appeared consistently changed by exposure to the MF diet during the first 12 months of life. IPA showed that this subset of 148 genes encompasses a large number of genes involved lipid and xenobiotic metabolic processes. The observation that PXR (or NR112) was the strongest predicted upstream regulator suggests a connection between these two functions. PXR is a ligand-activated nuclear receptor that, upon activation, forms a heterodimer with RXR. This complex is not only activated by exogenous toxins but has also been shown to responds to endobiotics like bile acids and steroid hormones [28]. Previous studies have shown that PXR activation in mice induces fatty acids uptake via up-regulation of $C d 36$ which is also one of the 148 consistently regulated genes. Additionally, other genes involved in lipid metabolism including 
Ppary, Cidea and Cidec are consistently up-regulated in the MF/INT diet switch mice. With respect to the PXR target genes involved in xenobiotic metabolism it should be noted that a large number of genes are consistently upregulated by the MF diet including Gstm1, Gstm5, Fmo1, Fmo2, Fmo3, Abcc3, Сур3a5, etc., indicating strongly enhanced xenobiotic and/or endobiotic metabolism in both the MF-exposed and the diet switch mice. Taken together these results suggest that, after a strong weight loss, robust reductions in the advanced stages of NAFLD can be achieved but that hepatic steatosis might not be fully reversible at old age.

In conclusion, our data indicate that intermittent CR offers significant health improving effects and largely counteracts the effects of a MF diet, but does not reach the health- and life span improving effects of a CR diet. Although the number of consistent molecular changes induced by a MF diet is small, they might have potentially important adverse effects on health. 


\section{EXPERIMENTAL PROCEDURES}

\section{Ethics statement}

The institutional and national guidelines for the care and use of animals were followed and the Local Committee for Care and Use of Laboratory Animals at Wageningen University approved the experiment (code number: drs-2010151b).

\section{Animals and diets}

Male C57BL/6J mice (age of 7 weeks) were purchased from Janvier (Cedex, France) and were housed in pairs of two in the light and temperature $\left(20^{\circ} \mathrm{C}\right)$-controlled animal facility of Wageningen University (12-hour light/dark cycle, light on at 04.00). The mice received standard AIN-93G (Research Diet Services, Wijk bij Duurstede, The Netherlands) for 2 weeks upon arrival.

At the start of the diet intervention the mice were 9 weeks old, housed individually and randomly distributed into four intervention groups: 1) Control diet (C) receiving AIN-93W diet ad libitum ( $\mathrm{n}=89$ ); 2) calorie restricted diet (CR) receiving AIN-93W-CR in portions containing 70E\% of the mean energy intake of the group of the control mice were provided each day at $15.30(n=117)$; 3) medium fat diet (MF) receiving AIN-93W-MF ad libitum ( $n=127)$; and (4) intermittent diet (INT) receiving alternating one week AIN-93W-MF ad libitum followed by one week 60E\% of control diet based on the mean energy intake of the mice on the AIN-93W diet $(n=155)$. AIN-93W-CR contained increased concentration of vitamins and minerals content in order to feed these mice the same concentrations of micronutrients as the mice receiving AIN-93W diet and avoid malnutrition. Complete diet composition is listed in Supplementary Table S3 (Research Diet Services, Wijk bij Duurstede, The Netherlands). All mice were provided with ad libitum access to water.

Body weight of all mice was recorded every two weeks. To represent a weekly body weight development, we weighed a smaller sample of mice of each intervention group every other week (20-24 mice). Food intake of 20 mice of each intervention group was measured every two months, comprising one week measurement for the C, CR and MF-fed mice and two weeks measurement for the INT-fed mice. Portion sizes of the mice on the CR and INT were adjusted at the beginning of the study and at the age of 6,12 and 18 months based on food intake of $C$ mice. At the age of 6, 12 and 24 months, 12-16 mice of each intervention group were sacrificed between 14.00-17.00 on 5 consecutive days (the remaining mice stayed in the experiment and were sacrificed at older ages). INT mice were sacrificed in their ad libitum MF feeding week. Similar to what we performed in the previous study [26], prior to sacrifice each mouse was first fasted for 4 hours after which they received an intragastric gavage of either solvent ( $0.5 \%$ carboxymethyl cellulose) or Wy-14,643 
dispersed in solvent (160 mg Wy-14,643/kg body weight), then fasted again for another 6 hours. in each dietary intervention group. In each diet group, the mice were paired according to bodyweight at sacrifice, so that mock and Wy-14,643 treatment were provided to mice with similar body weight. Only mock-treated animals were included in the molecular analysis, since the Wy-14,643 treatment have an immediate effect on the gene expression levels. The purpose of this treatment is to examine PPARa adaptive capacity analysis, which will be covered in a separate publication. After sedation with a mixture of isoflurane (1.5\%), nitrous oxide (70\%) and oxygen (30\%), blood samples were collected by cardiac puncture, then followed by neck dislocation. Weight of various organs was measured and subsequently organs/tissues were snap-frozen and stored at $-80^{\circ} \mathrm{C}$ until further molecular/biochemical analysis. For histological analysis, organs/tissues were fixed in $4 \%$ paraformaldehyde.

\section{Daily activity measurement}

At 23 months of age, a subset of mice were housed in new cages to monitor physical activity continuously as previously described [27, 29]. First mice were allowed to acclimatise for 3 days; followed by monitoring the activities of again 3 days. The results shown were averaged to dampen the day-to-day variability ( $n=3$ per group). Activity sensors (dual technology detector DUO 240, Visonic; adapted by R. Visser, NIN, Amsterdam, The Netherlands) were mounted above the cages and data were analysed with MED-PC ${ }^{\circledR}$ IV software for data collection (MED associates, St Albans, VT, USA). Activity was expressed in counts per $30 \mathrm{~min}$ (both for the total $24 \mathrm{~h}$ period, the dark period (active period) and the light period (inactive period)). Activity was calculated for each mouse separately. Statistical analyses were performed using SPSS 19.0 (SPSS Benelux, Gorinchem, the Netherlands) and differences were considered significant at a P-value below 0.05. Statistical analyses were performed on total daily activity data by use of a mixed model with post hoc LSD testing.

\section{DEXA scan body composition analysis}

Body composition was measured by Dual Energy X-ray Absorptiometry (DEXA) scan, using a PIXImus imager (GE Lunar, Madison, WI, USA). The scan produced data concerning lean mass, fat mass and bone mineral density. During the measurements the animals were under general anaesthesia (isoflurane $/ \mathrm{N}_{2} \mathrm{O} / \mathrm{O}_{2}$ ).

\section{Oral glucose tolerance test}

The mice sacrificed at the age of 23 months were all subjected to an oral glucose tolerance test (OGTT) two weeks prior to sacrifice. In the OGTT, the mice were fasted for 6 hours, then received 1.5 
mg glucose per gram body weight via an oral gavage. Subsequently, blood glucose was measured 15, 30, 45, 60, 90 and 150 minutes following the glucose load using Accu-Check blood glucose meters (Roche Diagnostics, Almere, The Netherlands).

\section{RNA isolation}

Total RNA was isolated using TRIzol reagent (Invitrogen Breda, The Netherlands) according to the manufacturer's instructions. The RNA was treated with DNAse and purified on columns using the RNAeasy microkit (Qiagen, Venlo, the Netherlands). RNA concentration was measured on a NanoDrop ND-1000 UV-vis spectrophotometer (Isogen, Maarssen, The Netherlands) and RNA integrity was checked on an Agilent 2100 Bioanalyzer (Agilent Technologies, Amsterdam, The Netherlands) with 6000 Nano Chips according to the manufacturer's instructions. RNA was judged as suitable only if samples showed intact bands of $18 \mathrm{~S}$ and $28 \mathrm{~S}$ ribosomal RNA subunits, displayed no chromosomal peaks or RNA degradation products, and had a RNA integrity number (RIN) above 8.0.

\section{Microarray hybridization and analysis}

$100 \mathrm{ng}$ of purified RNA was used for the preparation of labelled cDNA, applying the Ambion Whole Transcript (WT) Expression kit (Life Technologies, Carlsbad, USA) in combination with the Affymetrix GeneChip WT Terminal Labelling kit (Affymetrix, Santa Clara, USA). All samples were hybridized at one time point to Affymetrix GeneChip Mouse Gene 1.1 ST arrays according to standard Affymetrix protocols. Microarray analysis was performed in MADMAX, a pipeline for statistical analysis of microarray data. Arrays were normalized using the Robust Multiarray Average [30, 31]. Probe sets were defined according to Dai et al.[32]. In this method probes are assigned to unique gene identifiers, in this case Entrez IDs. The probes on the Gene 1.1 ST arrays represent 21,225 Entrez IDs. Array data will be submitted to the Gene Expression Omnibus after acceptance of the manuscript.

\section{Bioinformatic analysis}

Of the 21,225 defined genes covered by the MA, only genes with an intensity value of $\geq 20$ on at least 5 arrays, represented by at least 7 probes per gene on the array and an interquartile range $(I Q R) \geq 0.1$ were selected for further analysis and not annotated were removed. The top-1000 most variable genes were used for Principle Component Analysis (PCA) using MultiExperimentViewer version 4.8.1 [33, 34]. Signal $\log _{2}$ ratios, which represent fold changes (FC), and related significances of change were calculated from the mean signal intensities and differences between diet groups 
was analyzed using intensity based-moderated t-statistics (IBMT) implementing empirical Bayes correction [35]. Resulting $2 \log$ ratios and p-values were applied for further descriptive bioinformatic analysis of the data. Ingenuity Pathway Analysis (IPA, Ingenuity ${ }^{\circledR}$ Systems, www.ingenuity.com) was used to explore the canonical pathways affected by the 148 reversibly and 1510 consistently changed genes. Comparison of the expression patterns of the 148 irreversible changed genes in the MF, INT and MF/INT diet switch groups was carried out by generating a heat map using MultiExperimentViewer, version 4.8.1 [33, 34].

\section{Histopathology}

Formalin-fixed and paraffin-embedded cross-sections $(5 \mu \mathrm{m})$ of the liver lobe was stained with haematoxylin and eosin. Hepatic inflammation was assessed by counting the number of inflammatory foci at a $100 \times$ magnification (view size $3.1 \mathrm{~mm}^{2}$ ), in five non-overlapping fields.

\section{Hepatic triglyceride and 4-hydroxyproline measurement}

Liver triglycerides were determined in $5 \%$ liver homogenates prepared in buffer containing $250 \mathrm{mM}$ sucrose, $1 \mathrm{mM}$ EDTA, $10 \mathrm{mM}$ Tris- $\mathrm{HCl}(\mathrm{pH}$ 7.5), using the triglyceride Liquicolor Monoreagent (Instruchemie, Delfzijl, The Netherlands). 4-hydroxyproline content was determined spectrophotometrically in liver hydrolysates as previously described in Hillebrandt et al. [36].

\section{Plasma measurement}

Plasma insulin, resistin and PAI-1 levels were measured using a Mouse Adipokine (MADKMAG$71 \mathrm{~K})$ kit, according to the manufacturer's instructions. Plasma concentration of ALT was measured with commercially available kits from Instruchemie (Delfzijl, the Netherlands). Plasma insulin level was measured using a Mouse Adipokine (MADKMAG-71K) kit, according to the manufacturer's instructions. Plasma triglyceride and free fatty acid were measured using Liquicolor (Instruchemie, Wiesbaden, Germany) and NEFA-C kit (Wako, Neuss, Germany), respectively. Both assays were performed according to manufacturer's instructions.

\section{Statistical analysis}

Except for the gene expression, data were analysed with GraphPad Prism 5.04 applying 1-way ANOVA followed by a Tukey post-test analysis. Statistical significance for the survival of groups was established by the log-rank analysis of Kaplan-Meier plots. 


\section{ACKNOWLEDGEMENT}

This work was financially supported by the European Union's Seventh Framework Programme IDEAL (FP7/2007-2011) under grant agreement no: 259679.

\section{AUTHOR CONTRIBUTION}

Conceived and designed the experiments: FR, MM and WTS. Performed the experiments: FR, CL, MVB, MvD, ALW and WTS. Analyzed the data: WTS, FR, MVB, MvD, ALW, KvN. Assessed quality control of microarrays: MVB. Wrote the paper: FR and WTS. Provided valuable feedback on manuscript: KvN, MVB, MvD, ALW, MM. All authors read and approved the final manuscript.

\section{CONFLICT OF INTEREST STATEMENT}

Miriam van Dijk and Klaske Norren are affiliated with Nutricia Research. Aswin L Menke is affiliated with Triskelion. The other authors declare that they have no competing interests. 


\section{REFERENCES}

1. WHO. http://www.who.int/mediacentre/factsheets/fs311/en/. 2016; Available from: http://www.who.int/ mediacentre/factsheets/fs311/en/.

2. Sullivan, S., Implications of diet on nonalcoholic fatty liver disease. Curr Opin Gastroenterol, 2010, 26,1604.

3. Thoma, C., Day, C.P., and Trenell, M.I., Lifestyle interventions for the treatment of non-alcoholic fatty liver disease in adults: a systematic review. J Hepatol, 2012, 56,255-66.

4. Fontana, L., Partridge, L., and Longo, V.D., Extending healthy life span--from yeast to humans. Science, 2010, 328,321-6.

5. Fontana, L. and Partridge, L., Promoting health and longevity through diet: from model organisms to humans. Cell, 2015, 161,106-18.

6. Speakman, J.R. and Mitchell, S.E., Caloric restriction. Mol Aspects Med, 2011, 32,159-221.

7. Fairburn, C.G. and Harrison, P.J., Eating disorders. Lancet, 2003, 361,407-16.

8. Hunt, N.D., Li, G.D., Zhu, M., Miller, M., et al., Effect of calorie restriction and refeeding on skin wound healing in the rat. Age (Dordr), 2012, 34,1453-8.

9. Kristan, D.M., Calorie restriction and susceptibility to intact pathogens. Age (Dordr), 2008, 30,147-56.

10. Martin, B., Pearson, M., Kebejian, L., Golden, E., et al., Sex-dependent metabolic, neuroendocrine, and cognitive responses to dietary energy restriction and excess. Endocrinology, 2007, 148,4318-33.

11. Brandhorst, S., Choi, I.Y., Wei, M., Cheng, C.W., et al., A Periodic Diet that Mimics Fasting Promotes MultiSystem Regeneration, Enhanced Cognitive Performance, and Healthspan. Cell metabolism, 2015, 22,86-99.

12. Longo, V.D. and Mattson, M.P., Fasting: molecular mechanisms and clinical applications. Cell metabolism, 2014, 19,181-92.

13. Rothschild, J., Hoddy, K.K., Jambazian, P., and Varady, K.A., Time-restricted feeding and risk of metabolic disease: a review of human and animal studies. Nutr Rev, 2014, 72,308-18.

14. Calabrese, E.J. and Baldwin, L.A., Defining hormesis. Human \& experimental toxicology, 2002, 21,91-7.

15. Rattan, S.I., Principles and practice of hormetic treatment of aging and age-related diseases. Human \& experimental toxicology, 2008, 27,151-4.

16. Chaix, A., Zarrinpar, A., Miu, P., and Panda, S., Time-restricted feeding is a preventative and therapeutic intervention against diverse nutritional challenges. Cell metabolism, 2014, 20,991-1005.

17. Mattson, M.P., Challenging oneself intermittently to improve health. Dose-response : a publication of International Hormesis Society, 2014, 12,600-18.

18. Mattson, M.P., Allison, D.B., Fontana, L., Harvie, M., et al., Meal frequency and timing in health and disease. Proc Natl Acad Sci U S A, 2014, 111,16647-53.

19. Fisher, C.P., Kierzek, A.M., Plant, N.J., and Moore, J.B., Systems biology approaches for studying the pathogenesis of non-alcoholic fatty liver disease. World J Gastroenterol, 2014, 20,15070-8.

20. Pais, R., Charlotte, F., Fedchuk, L., Bedossa, P., et al., A systematic review of follow-up biopsies reveals disease progression in patients with non-alcoholic fatty liver. J Hepatol, 2013, 59,550-6.

21. De Minicis, S., Day, C., and Svegliati-Baroni, G., From NAFLD to NASH and HCC: pathogenetic mechanisms and therapeutic insights. Curr Pharm Des, 2013, 19,5239-49.

22. Argo, C.K. and Caldwell, S.H., Epidemiology and natural history of non-alcoholic steatohepatitis. Clinics in liver disease, 2009, 13,511-31.

23. Gan, L., Chitturi, S., and Farrell, G.C., Mechanisms and implications of age-related changes in the liver: nonalcoholic Fatty liver disease in the elderly. Current gerontology and geriatrics research, 2011, 2011,831536 . 
24. Sheedfar, F., Di Biase, S., Koonen, D., and Vinciguerra, M., Liver diseases and aging: friends or foes? Aging Cell, 2013, 12,950-4.

25. Tajiri, K. and Shimizu, Y., Liver physiology and liver diseases in the elderly. World J Gastroenterol, 2013, 19,8459-67.

26. Rusli, F., Boekschoten, M.V., Zubia, A.A., Lute, C., et al., A weekly alternating diet between caloric restriction and medium fat protects the liver from fatty liver development in middle-aged C57BL/6J mice. Mol Nutr Food Res, 2014.

27. van Norren, K., Rusli, F., van Dijk, M., Lute, C., et al., Behavioural changes are a major contributing factor in the reduction of sarcopenia in caloric-restricted ageing mice. Journal of cachexia, sarcopenia and muscle, 2015, 6,253-68.

28. Naik, A., Belic, A., Zanger, U.M., and Rozman, D., Molecular Interactions between NAFLD and Xenobiotic Metabolism. Frontiers in genetics, 2013, 4,2.

29. van Dijk, M., Dijk, F.J., Bunschoten, A., van Dartel, D.A., et al., Improved muscle function and quality after diet intervention with leucine-enriched whey and antioxidants in antioxidant deficient aged mice. Oncotarget, 2016.

30. Bolstad, B.M., Irizarry, R.A., Astrand, M., and Speed, T.P., A comparison of normalization methods for high density oligonucleotide array data based on variance and bias. Bioinformatics, 2003, 19,185-93.

31. Irizarry, R.A., Bolstad, B.M., Collin, F., Cope, L.M., et al., Summaries of Affymetrix GeneChip probe level data. Nucleic Acids Res, 2003, 31 ,e15.

32. Dai, M., Wang, P., Boyd, A.D., Kostov, G., et al., Evolving gene/transcript definitions significantly alter the interpretation of GeneChip data. Nucleic Acids Research, 2005, 33,e175.

33. Saeed, A.I., Bhagabati, N.K., Braisted, J.C., Liang, W., et al., TM4 microarray software suite. Methods Enzymol, 2006, 411,134-93.

34. Saeed, A.I., Sharov, V., White, J., Li, J., et al., TM4: a free, open-source system for microarray data management and analysis. BioTechniques, 2003, 34,374-378.

35. Sartor, M.A., Tomlinson, C.R., Wesselkamper, S.C., Sivaganesan, S., et al., Intensity-based hierarchical Bayes method improves testing for differentially expressed genes in microarray experiments. BMC Bioinformatics, 2006, 7,538.

36. Hillebrandt, S., Wasmuth, H.E., Weiskirchen, R., Hellerbrand, C., et al., Complement factor 5 is a quantitative trait gene that modifies liver fibrogenesis in mice and humans. Nat Genet, 2005, 37,835-43. 


\section{SUPPLEMENTARY TABLES}

Supplementary Table S1. 148 genes displaying differential expression between MF and INTexposed mice revealing that do not adapt to the INT diet in the MF/INT diet switch group

MF vs INT

\begin{tabular}{|c|c|c|c|c|c|c|}
\hline Symbol & FC & p-value & FC & p-value & FC & p-value \\
\hline Hsd3b5 & -25.23 & 1.09E-06 & 1.87 & 3.09E-01 & -13.47 & $9.31 \mathrm{E}-05$ \\
\hline Serpina4-ps1 & -7.24 & $5.08 \mathrm{E}-06$ & 1.64 & $2.26 \mathrm{E}-01$ & -4.42 & 6.43E-04 \\
\hline Susd4 & -4.08 & $2.06 \mathrm{E}-06$ & 1.69 & $5.91 \mathrm{E}-02$ & -2.41 & 2.69E-03 \\
\hline Gpr110 & -3.39 & 2.05E-04 & 1.20 & $5.70 \mathrm{E}-01$ & -2.84 & $1.88 \mathrm{E}-03$ \\
\hline C4a & -3.09 & $1.93 \mathrm{E}-07$ & 1.60 & $2.02 \mathrm{E}-02$ & -1.93 & $1.88 \mathrm{E}-03$ \\
\hline Slc22a28 & -2.84 & $6.23 \mathrm{E}-05$ & 1.34 & $2.36 \mathrm{E}-01$ & -2.12 & 4.36E-03 \\
\hline Cyp7b1 & -2.32 & 5.17E-05 & 1.24 & $2.76 \mathrm{E}-01$ & -1.87 & $2.86 \mathrm{E}-03$ \\
\hline Sult5al & -2.32 & $1.39 \mathrm{E}-07$ & 1.33 & $5.48 \mathrm{E}-02$ & -1.74 & $3.94 \mathrm{E}-04$ \\
\hline Grm8 & -2.25 & $2.69 \mathrm{E}-07$ & 1.31 & $6.68 \mathrm{E}-02$ & -1.72 & $4.88 \mathrm{E}-04$ \\
\hline Mup21 & -2.17 & 1.27E-03 & 1.07 & $7.60 \mathrm{E}-01$ & -2.02 & 4.32E-03 \\
\hline Slc22a7 & -2.15 & $9.34 \mathrm{E}-04$ & 1.07 & 7.59E-01 & -2.00 & 3.29E-03 \\
\hline Serpine2 & -2.01 & $6.47 \mathrm{E}-05$ & 1.20 & $2.79 \mathrm{E}-01$ & -1.68 & 3.34E-03 \\
\hline Cabyr & -1.95 & 3.23E-03 & -1.01 & $9.73 \mathrm{E}-01$ & -1.97 & $3.90 \mathrm{E}-03$ \\
\hline Olfr541 & -1.95 & $3.66 \mathrm{E}-07$ & 1.31 & 2.91E-02 & -1.49 & $1.91 \mathrm{E}-03$ \\
\hline Adh6-psl & -1.79 & 1.57E-03 & 1.10 & $5.86 \mathrm{E}-01$ & -1.62 & $9.98 \mathrm{E}-03$ \\
\hline Cyp21a1 & -1.78 & $2.29 \mathrm{E}-03$ & 1.07 & $6.96 \mathrm{E}-01$ & -1.65 & $9.16 \mathrm{E}-03$ \\
\hline Lama3 & -1.72 & 4.77E-07 & 1.12 & $2.72 \mathrm{E}-01$ & -1.54 & $6.14 \mathrm{E}-05$ \\
\hline Ttc39c & -1.63 & $7.15 \mathrm{E}-05$ & 1.03 & $8.25 \mathrm{E}-01$ & -1.59 & 2.44E-04 \\
\hline Lrrcl6a & -1.62 & $3.16 \mathrm{E}-03$ & 1.02 & $9.23 \mathrm{E}-01$ & -1.59 & $5.51 \mathrm{E}-03$ \\
\hline Igfbp2 & -1.58 & $6.43 \mathrm{E}-04$ & 1.07 & $5.90 \mathrm{E}-01$ & -1.47 & 4.62E-03 \\
\hline Pcolce2 & -1.55 & 3.77E-04 & 1.01 & $9.38 \mathrm{E}-01$ & -1.54 & $7.18 \mathrm{E}-04$ \\
\hline Tspan33 & -1.55 & 5.51E-05 & 1.11 & $3.00 \mathrm{E}-01$ & -1.39 & $2.56 \mathrm{E}-03$ \\
\hline Slco2al & -1.52 & $1.91 \mathrm{E}-04$ & 1.11 & $3.39 \mathrm{E}-01$ & -1.37 & $5.50 \mathrm{E}-03$ \\
\hline$c 8 b$ & -1.51 & $1.14 \mathrm{E}-03$ & -1.05 & $7.10 \mathrm{E}-01$ & -1.58 & 4.98E-04 \\
\hline Hes6 & -1.49 & $1.51 \mathrm{E}-03$ & 1.04 & 7.50E-01 & -1.44 & $5.23 \mathrm{E}-03$ \\
\hline Slc30a3 & -1.47 & $2.16 \mathrm{E}-05$ & 1.13 & $1.53 \mathrm{E}-01$ & -1.30 & $3.91 \mathrm{E}-03$ \\
\hline Alas2 & -1.46 & $6.49 \mathrm{E}-05$ & 1.05 & $6.09 \mathrm{E}-01$ & -1.39 & $5.80 \mathrm{E}-04$ \\
\hline Ccbll & -1.45 & $1.24 \mathrm{E}-04$ & 1.04 & $6.82 \mathrm{E}-01$ & -1.39 & 7.44E-04 \\
\hline Ppp1 r9a & -1.43 & $2.26 \mathrm{E}-03$ & 1.01 & $9.13 \mathrm{E}-01$ & -1.41 & 4.19E-03 \\
\hline$R d 3$ & -1.41 & $3.06 \mathrm{E}-06$ & 1.13 & 7.93E-02 & -1.25 & 2.40E-03 \\
\hline Adrb3 & -1.40 & $6.21 \mathrm{E}-03$ & -1.01 & $9.51 \mathrm{E}-01$ & -1.41 & $6.76 \mathrm{E}-03$ \\
\hline Gm4981 & -1.39 & $4.91 \mathrm{E}-04$ & 1.02 & $8.42 \mathrm{E}-01$ & -1.36 & 1.34E-03 \\
\hline Сур2c44 & -1.37 & $4.20 \mathrm{E}-04$ & 1.05 & $5.92 \mathrm{E}-01$ & -1.31 & $3.18 \mathrm{E}-03$ \\
\hline Gm3934 & -1.36 & $1.22 \mathrm{E}-03$ & -1.03 & 7.89E-01 & -1.40 & 7.42E-04 \\
\hline Tle6 & -1.36 & $1.80 \mathrm{E}-04$ & 1.04 & $6.08 \mathrm{E}-01$ & -1.30 & 1.43E-03 \\
\hline Gstp1 & -1.35 & $3.94 \mathrm{E}-04$ & -1.02 & $8.46 \mathrm{E}-01$ & -1.38 & 3.09E-04 \\
\hline Serpina11 & -1.35 & $5.26 \mathrm{E}-04$ & -1.04 & $6.58 \mathrm{E}-01$ & -1.40 & 1.79E-04 \\
\hline Dnasel & -1.32 & $1.78 \mathrm{E}-05$ & 1.06 & $3.45 \mathrm{E}-01$ & -1.25 & 7.69E-04 \\
\hline Irf6 & -1.31 & $2.58 \mathrm{E}-03$ & -1.01 & 9.34E-01 & -1.32 & 2.73E-03 \\
\hline Gcat & -1.30 & $1.36 \mathrm{E}-04$ & 1.08 & $2.78 \mathrm{E}-01$ & -1.21 & 6.01E-03 \\
\hline Srgap3 & -1.30 & $3.18 \mathrm{E}-03$ & -1.01 & $8.93 \mathrm{E}-01$ & -1.31 & $2.87 \mathrm{E}-03$ \\
\hline Tiaf2 & -1.29 & 4.44E-04 & 1.05 & $5.24 \mathrm{E}-01$ & -1.23 & 4.45E-03 \\
\hline Tdrkh & -1.28 & $1.75 \mathrm{E}-03$ & -1.01 & $8.52 \mathrm{E}-01$ & -1.30 & 1.37E-03 \\
\hline
\end{tabular}


Supplementary Table S1. (Continued)

\begin{tabular}{|c|c|c|c|c|c|c|}
\hline \multirow[b]{2}{*}{ Symbol } & \multicolumn{2}{|c|}{ MF vs INT } & \multicolumn{2}{|c|}{ MF/INT vs MF } & \multicolumn{2}{|c|}{ MF/INT vs INT } \\
\hline & FC & p-value & FC & p-value & FC & p-value \\
\hline Apom & -1.27 & $1.33 \mathrm{E}-03$ & -1.05 & $5.23 \mathrm{E}-01$ & -1.32 & $2.41 \mathrm{E}-04$ \\
\hline Cbs & -1.25 & $1.36 \mathrm{E}-03$ & -1.02 & $7.58 \mathrm{E}-01$ & -1.28 & 7.31E-04 \\
\hline Tnik & -1.24 & 6.80E-03 & -1.03 & 7.19E-01 & -1.28 & 3.19E-03 \\
\hline Smarcd2 & -1.23 & 1.19E-04 & 1.06 & $2.41 \mathrm{E}-01$ & -1.16 & $6.92 \mathrm{E}-03$ \\
\hline Cwf19l1 & -1.23 & 2.37E-04 & 1.04 & 4.55E-01 & -1.18 & $3.60 \mathrm{E}-03$ \\
\hline D830014E11Rik & -1.23 & $3.16 \mathrm{E}-03$ & -1.02 & $8.15 \mathrm{E}-01$ & -1.25 & $2.13 \mathrm{E}-03$ \\
\hline Slc19a2 & -1.22 & $2.86 \mathrm{E}-03$ & -1.05 & 4.66E-01 & -1.28 & 4.07E-04 \\
\hline Gm10766 & -1.21 & $5.27 \mathrm{E}-03$ & 1.00 & 9.87E-01 & -1.21 & 7.14E-03 \\
\hline Gabrd & -1.21 & $1.48 \mathrm{E}-03$ & 1.03 & $5.89 \mathrm{E}-01$ & -1.18 & $9.38 \mathrm{E}-03$ \\
\hline Bmp2 & -1.21 & $2.32 \mathrm{E}-03$ & 1.02 & $7.50 \mathrm{E}-01$ & -1.19 & $7.63 \mathrm{E}-03$ \\
\hline$K d r$ & -1.21 & $1.20 \mathrm{E}-04$ & 1.06 & $2.47 \mathrm{E}-01$ & -1.15 & $6.68 \mathrm{E}-03$ \\
\hline Apobec2 & -1.21 & $5.21 \mathrm{E}-03$ & -1.01 & $8.73 E-01$ & -1.22 & 4.33E-03 \\
\hline Gcn1ll & -1.21 & $2.82 \mathrm{E}-05$ & 1.07 & $1.18 \mathrm{E}-01$ & -1.13 & 6.97E-03 \\
\hline Porcn & -1.20 & $6.41 \mathrm{E}-03$ & -1.02 & $7.89 \mathrm{E}-01$ & -1.23 & $3.91 \mathrm{E}-03$ \\
\hline Scarf1 & -1.20 & 4.33E-03 & -1.02 & $6.94 \mathrm{E}-01$ & -1.23 & $1.82 \mathrm{E}-03$ \\
\hline Tmem63a & -1.20 & 1.09E-03 & 1.01 & $8.25 \mathrm{E}-01$ & -1.18 & $2.95 \mathrm{E}-03$ \\
\hline Ppplrla & -1.19 & $9.66 \mathrm{E}-05$ & 1.04 & $3.31 \mathrm{E}-01$ & -1.14 & 3.33E-03 \\
\hline Gtf2h4 & -1.19 & $5.31 \mathrm{E}-04$ & 1.03 & 5.03E-01 & -1.15 & $5.67 \mathrm{E}-03$ \\
\hline Sun2 & -1.18 & 1.79E-03 & -1.05 & 3.47E-01 & -1.24 & $1.20 \mathrm{E}-04$ \\
\hline Exoc3l & -1.18 & $3.91 \mathrm{E}-03$ & -1.01 & $8.31 \mathrm{E}-01$ & -1.19 & $2.80 \mathrm{E}-03$ \\
\hline Adcy 4 & -1.18 & $6.82 \mathrm{E}-03$ & -1.00 & 9.87E-01 & -1.18 & 8.34E-03 \\
\hline Fgf8 & -1.18 & 3.17E-03 & 1.01 & $8.23 \mathrm{E}-01$ & -1.16 & 7.82E-03 \\
\hline Repin1 & -1.17 & 1.17E-03 & -1.05 & $3.50 \mathrm{E}-01$ & -1.22 & 7.63E-05 \\
\hline$R b f a$ & -1.17 & $5.50 \mathrm{E}-04$ & 1.03 & 4.72E-01 & -1.13 & $6.68 \mathrm{E}-03$ \\
\hline Pskh1 & -1.16 & 4.82E-05 & 1.03 & $3.75 \mathrm{E}-01$ & -1.13 & $1.47 \mathrm{E}-03$ \\
\hline Sil1 & -1.15 & 4.17E-04 & 1.03 & 5.19E-01 & -1.12 & 4.32E-03 \\
\hline Nelfe & -1.15 & 6.65E-03 & -1.02 & $6.40 \mathrm{E}-01$ & -1.18 & $2.28 \mathrm{E}-03$ \\
\hline Skiv2l & -1.14 & 8.84E-05 & 1.04 & $1.92 \mathrm{E}-01$ & -1.09 & 7.93E-03 \\
\hline Os9 & -1.13 & 3.93E-04 & -1.01 & 8.47E-01 & -1.14 & $3.10 \mathrm{E}-04$ \\
\hline Irf3 & -1.13 & 8.83E-04 & 1.02 & $5.53 \mathrm{E}-01$ & -1.11 & $7.01 \mathrm{E}-03$ \\
\hline Arid1 $b$ & -1.09 & 8.59E-03 & -1.02 & $5.27 \mathrm{E}-01$ & -1.11 & $1.83 \mathrm{E}-03$ \\
\hline Angptl3 & 1.13 & 4.33E-04 & -1.02 & $6.52 \mathrm{E}-01$ & 1.11 & $2.54 \mathrm{E}-03$ \\
\hline Arf4 & 1.16 & $2.75 \mathrm{E}-03$ & -1.01 & 8.89E-01 & 1.15 & $5.47 \mathrm{E}-03$ \\
\hline Fmol & 1.16 & 7.29E-03 & 1.04 & 4.84E-01 & 1.20 & $1.24 \mathrm{E}-03$ \\
\hline Gbel & 1.16 & $8.50 \mathrm{E}-03$ & 1.01 & $8.80 \mathrm{E}-01$ & 1.17 & 7.17E-03 \\
\hline Tcn2 & 1.17 & $5.55 \mathrm{E}-04$ & -1.03 & 4.66E-01 & 1.14 & $6.91 \mathrm{E}-03$ \\
\hline Gstm1 & 1.18 & $2.70 \mathrm{E}-03$ & -1.01 & $9.14 \mathrm{E}-01$ & 1.17 & 4.91E-03 \\
\hline Kif18a & 1.18 & $9.76 \mathrm{E}-03$ & 1.03 & $6.92 \mathrm{E}-01$ & 1.22 & $4.18 \mathrm{E}-03$ \\
\hline Setd8 & 1.19 & 8.54E-03 & 1.03 & $6.01 \mathrm{E}-01$ & 1.23 & $2.52 \mathrm{E}-03$ \\
\hline Bche & 1.20 & $3.15 \mathrm{E}-03$ & 1.04 & $5.10 \mathrm{E}-01$ & 1.25 & $5.69 \mathrm{E}-04$ \\
\hline Rny3 & 1.20 & $4.18 \mathrm{E}-04$ & -1.02 & $7.21 \mathrm{E}-01$ & 1.18 & $1.87 \mathrm{E}-03$ \\
\hline Megf9 & 1.21 & 7.00E-03 & 1.01 & $9.01 \mathrm{E}-01$ & 1.22 & 6.38E-03 \\
\hline Slc16a7 & 1.22 & $5.75 \mathrm{E}-03$ & 1.10 & $1.71 \mathrm{E}-01$ & 1.34 & $1.03 \mathrm{E}-04$ \\
\hline
\end{tabular}


Supplementary Table S1. (Continued)

MF vs INT

\begin{tabular}{|c|c|c|c|c|c|c|}
\hline Symbol & FC & p-value & FC & p-value & FC & p-value \\
\hline Cdk6 & 1.22 & 3.29E-03 & 1.04 & $5.98 \mathrm{E}-01$ & 1.27 & $9.03 \mathrm{E}-04$ \\
\hline Fut8 & 1.22 & 1.43E-03 & 1.03 & 5.79E-01 & 1.27 & 3.44E-04 \\
\hline Fkbpla & 1.23 & $3.86 \mathrm{E}-03$ & -1.01 & $8.68 \mathrm{E}-01$ & 1.21 & $8.01 \mathrm{E}-03$ \\
\hline $\operatorname{Prdx} 3$ & 1.24 & $6.93 \mathrm{E}-06$ & -1.08 & 1.10E-01 & 1.16 & 2.77E-03 \\
\hline Syce2 & 1.25 & 1.88E-03 & 1.01 & $8.61 \mathrm{E}-01$ & 1.26 & $1.52 \mathrm{E}-03$ \\
\hline Tmc6 & 1.25 & 2.67E-04 & -1.03 & 6.42E-01 & 1.22 & $1.74 \mathrm{E}-03$ \\
\hline Slc9ab & 1.26 & 7.12E-04 & -1.04 & 5.54E-01 & 1.21 & $5.83 \mathrm{E}-03$ \\
\hline Cers6 & 1.29 & $8.61 \mathrm{E}-03$ & 1.09 & 3.57E-01 & 1.41 & $7.48 \mathrm{E}-04$ \\
\hline Impact & 1.30 & 2.09E-05 & -1.11 & 8.78E-02 & 1.18 & 8.39E-03 \\
\hline$A b c c 3$ & 1.30 & 7.03E-03 & 1.01 & $9.14 \mathrm{E}-01$ & 1.32 & 6.69E-03 \\
\hline Ugt8a & 1.31 & 5.34E-04 & -1.03 & 7.22E-01 & 1.28 & $2.31 \mathrm{E}-03$ \\
\hline Acer2 & 1.32 & 1.15E-03 & -1.04 & 6.70E-01 & 1.27 & $5.53 \mathrm{E}-03$ \\
\hline Osgin2 & 1.35 & 7.76E-04 & -1.00 & $9.91 \mathrm{E}-01$ & 1.35 & 1.15E-03 \\
\hline Rtn4 & 1.38 & 4.64E-04 & -1.06 & $5.12 \mathrm{E}-01$ & 1.30 & 4.87E-03 \\
\hline$V w f$ & 1.38 & 3.00E-07 & -1.11 & 6.72E-02 & 1.24 & $5.27 E-04$ \\
\hline Dzip1l & 1.38 & 2.97E-07 & -1.15 & 1.64E-02 & 1.20 & $3.21 \mathrm{E}-03$ \\
\hline $\mathrm{Nebl}$ & 1.41 & 1.60E-03 & -1.01 & $9.31 \mathrm{E}-01$ & 1.40 & $2.85 \mathrm{E}-03$ \\
\hline Сур3а59 & 1.42 & 6.10E-03 & 1.14 & 2.94E-01 & 1.61 & $3.31 \mathrm{E}-04$ \\
\hline Vldlr & 1.43 & $9.94 \mathrm{E}-03$ & 1.12 & $3.98 \mathrm{E}-01$ & 1.60 & $1.12 \mathrm{E}-03$ \\
\hline Selp & 1.43 & 6.54E-05 & -1.05 & 5.54E-01 & 1.36 & $7.55 \mathrm{E}-04$ \\
\hline Fam19a2 & 1.44 & 2.19E-04 & 1.01 & 8.82E-01 & 1.46 & $2.04 E-04$ \\
\hline Clqtnf7 & 1.45 & $2.20 \mathrm{E}-05$ & -1.15 & $1.04 \mathrm{E}-01$ & 1.27 & $7.00 \mathrm{E}-03$ \\
\hline Spal7 & 1.45 & 1.17E-05 & -1.17 & 5.64E-02 & 1.24 & $9.71 \mathrm{E}-03$ \\
\hline Zfp521 & 1.46 & 1.17E-06 & -1.19 & $1.56 \mathrm{E}-02$ & 1.22 & $8.54 \mathrm{E}-03$ \\
\hline Aebpl & 1.48 & $3.40 \mathrm{E}-05$ & -1.14 & $1.41 \mathrm{E}-01$ & 1.29 & $6.22 \mathrm{E}-03$ \\
\hline Prelid2 & 1.51 & 1.77E-03 & 1.06 & 6.39E-01 & 1.61 & $5.73 \mathrm{E}-04$ \\
\hline $\operatorname{Tm} 4 s f 4$ & 1.53 & 7.81E-05 & -1.12 & 2.67E-01 & 1.36 & 4.20E-03 \\
\hline Plagl1 & 1.54 & 8.44E-06 & -1.19 & $5.81 \mathrm{E}-02$ & 1.29 & $7.48 \mathrm{E}-03$ \\
\hline Gstm2 & 1.54 & $6.56 \mathrm{E}-05$ & -1.15 & 1.67E-01 & 1.34 & $7.83 \mathrm{E}-03$ \\
\hline Gas6 & 1.55 & 5.19E-03 & 1.09 & 5.84E-01 & 1.69 & $1.37 \mathrm{E}-03$ \\
\hline Glod5 & 1.56 & 5.29E-05 & -1.13 & 2.57E-01 & 1.38 & $3.31 \mathrm{E}-03$ \\
\hline Сур3a11 & 1.58 & 3.49E-08 & -1.11 & 1.79E-01 & 1.43 & $1.61 \mathrm{E}-05$ \\
\hline Rsph4a & 1.59 & $6.85 \mathrm{E}-03$ & 1.46 & 2.60E-02 & 2.31 & $5.14 \mathrm{E}-06$ \\
\hline Nqol & 1.60 & 5.57E-03 & 1.03 & 8.63E-01 & 1.64 & 4.45E-03 \\
\hline Gm10872 & 1.65 & $2.15 \mathrm{E}-03$ & 1.02 & $9.24 \mathrm{E}-01$ & 1.68 & $2.21 \mathrm{E}-03$ \\
\hline$A b c b l a$ & 1.69 & $5.06 \mathrm{E}-05$ & 1.00 & $9.96 \mathrm{E}-01$ & 1.69 & $8.15 \mathrm{E}-05$ \\
\hline Chil1 & 1.71 & $2.13 \mathrm{E}-03$ & 1.02 & $9.03 \mathrm{E}-01$ & 1.74 & $2.02 \mathrm{E}-03$ \\
\hline Pparg & 1.76 & $5.08 \mathrm{E}-04$ & -1.07 & $6.61 \mathrm{E}-01$ & 1.64 & $2.82 \mathrm{E}-03$ \\
\hline Tmem45b & 1.90 & 1.64E-03 & 1.10 & $6.26 \mathrm{E}-01$ & 2.10 & 4.98E-04 \\
\hline Сур2с39 & 1.96 & $1.35 \mathrm{E}-04$ & 1.08 & 6.50E-01 & 2.11 & 4.35E-05 \\
\hline Corin & 1.96 & $1.68 \mathrm{E}-05$ & -1.16 & $3.14 \mathrm{E}-01$ & 1.69 & $8.90 \mathrm{E}-04$ \\
\hline$C d 36$ & 2.03 & $9.12 \mathrm{E}-04$ & 1.02 & $9.38 \mathrm{E}-01$ & 2.06 & $1.02 \mathrm{E}-03$ \\
\hline Tceal8 & 2.03 & $1.12 \mathrm{E}-06$ & -1.22 & $1.38 \mathrm{E}-01$ & 1.66 & 4.69E-04 \\
\hline
\end{tabular}


Supplementary Table S1. (Continued)

\begin{tabular}{l|cc|cc|cc}
\multicolumn{2}{c}{} & \multicolumn{2}{c}{ MF vs INT } & \multicolumn{2}{c}{ MF/INT vs MF } & \multicolumn{2}{c}{ MF/INT vs INT } \\
Symbol & FC & p-value & FC & p-value & FC & p-value \\
\hline Plin4 & 2.04 & $2.03 \mathrm{E}-03$ & -1.04 & $8.46 \mathrm{E}-01$ & 1.95 & $4.83 \mathrm{E}-03$ \\
Mbn13 & 2.08 & $4.70 \mathrm{E}-06$ & -1.15 & $3.67 \mathrm{E}-01$ & 1.82 & $2.18 \mathrm{E}-04$ \\
Rcan2 & 2.16 & $5.36 \mathrm{E}-06$ & -1.07 & $6.66 \mathrm{E}-01$ & 2.01 & $4.76 \mathrm{E}-05$ \\
Lgals1 & 2.21 & $8.17 \mathrm{E}-05$ & -1.12 & $5.68 \mathrm{E}-01$ & 1.98 & $8.59 \mathrm{E}-04$ \\
Fam83a & 2.41 & $3.88 \mathrm{E}-07$ & -1.39 & $4.34 \mathrm{E}-02$ & 1.74 & $1.19 \mathrm{E}-03$ \\
Ttc39a & 2.41 & $6.03 \mathrm{E}-08$ & -1.27 & $1.15 \mathrm{E}-01$ & 1.91 & $6.02 \mathrm{E}-05$ \\
Fmo2 & 2.43 & $8.24 \mathrm{E}-07$ & -1.19 & $3.03 \mathrm{E}-01$ & 2.04 & $7.69 \mathrm{E}-05$ \\
Orm3 & 2.47 & $4.08 \mathrm{E}-03$ & 1.23 & $4.99 \mathrm{E}-01$ & 3.03 & $7.11 \mathrm{E}-04$ \\
S100a11 & 2.70 & $1.25 \mathrm{E}-04$ & -1.31 & $2.73 \mathrm{E}-01$ & 2.05 & $5.80 \mathrm{E}-03$ \\
Gprc5b & 2.78 & $1.77 \mathrm{E}-05$ & -1.06 & $8.03 \mathrm{E}-01$ & 2.63 & $7.43 \mathrm{E}-05$ \\
Themis & 2.91 & $4.06 \mathrm{E}-05$ & -1.22 & $4.28 \mathrm{E}-01$ & 2.39 & $9.49 \mathrm{E}-04$ \\
Cyp2a22 & 3.00 & $1.31 \mathrm{E}-03$ & 1.24 & $5.23 \mathrm{E}-01$ & 3.70 & $2.37 \mathrm{E}-04$ \\
Cidec & 3.85 & $2.00 \mathrm{E}-04$ & -1.32 & $4.22 \mathrm{E}-01$ & 2.91 & $3.70 \mathrm{E}-03$ \\
Fmo3 & 4.22 & $2.19 \mathrm{E}-07$ & -1.60 & $6.74 \mathrm{E}-02$ & 2.63 & $4.11 \mathrm{E}-04$ \\
B430212C06Rik & 4.41 & $4.85 \mathrm{E}-07$ & 1.09 & $7.62 \mathrm{E}-01$ & 4.78 & $2.95 \mathrm{E}-07$ \\
Cidea & 5.08 & $2.57 \mathrm{E}-05$ & 1.39 & $3.73 \mathrm{E}-01$ & 7.04 & $1.52 \mathrm{E}-06$ \\
Cyp2b13 & 9.81 & $1.16 \mathrm{E}-10$ & 1.06 & $8.48 \mathrm{E}-01$ & 10.41 & $1.45 \mathrm{E}-10$
\end{tabular}


Supplementary Table S2. Upstream regulators of the $148 \mathrm{MF}$-consistent genes with a $\mathrm{p}$-value of overlap $<0.001$ and an activation score $>2.00$ or repression score $<-2.00$

\begin{tabular}{|c|c|c|c|c|c|}
\hline $\begin{array}{l}\text { Upstream } \\
\text { Regulator }\end{array}$ & $\begin{array}{l}\text { Exp. } \\
\text { Fold } \\
\text { Change }\end{array}$ & Molecule Type & $\begin{array}{l}\text { Predicted } \\
\text { Activation } \\
\text { State }\end{array}$ & $\begin{array}{l}\text { Activation } \\
\text { z-score }\end{array}$ & $\begin{array}{l}\text { p-value of } \\
\text { overlap }\end{array}$ \\
\hline PXR & & $\begin{array}{l}\text { ligand-dependent } \\
\text { nuclear receptor }\end{array}$ & Activated & 3.20 & $2.28 \mathrm{E}-13$ \\
\hline Ncoa-PXR-Rxra & & complex & Activated & 2.00 & 4.50E-06 \\
\hline $\begin{array}{l}\text { PXR ligand-PXR- } \\
\text { Retinoic acid-RXRa }\end{array}$ & & complex & Activated & 2.20 & $4.58 \mathrm{E}-06$ \\
\hline MED13 & & $\begin{array}{l}\text { transcription } \\
\text { regulator }\end{array}$ & Inhibited & -2.00 & $8.38 \mathrm{E}-06$ \\
\hline NFE2L2 & & $\begin{array}{l}\text { transcription } \\
\text { regulator }\end{array}$ & Activated & 2.80 & 2.83E-05 \\
\hline PPARG & 1.76 & $\begin{array}{l}\text { ligand-dependent } \\
\text { nuclear receptor }\end{array}$ & Activated & 2.60 & 4.00E-04 \\
\hline mir-223 & & microRNA & Activated & 2.00 & $9.24 \mathrm{E}-04$ \\
\hline
\end{tabular}

Supplementary Table S3. Composition of the experimental diet. The CR diet was adjusted for the vitamins and minerals amount to ensure a homologous intake between both groups.

\begin{tabular}{|c|c|c|c|}
\hline & AIN-93W & AIN-93W-CR & AIN-93W-MF \\
\hline Energy $(\mathrm{kcal} / \mathrm{g})$ & 3.85 & 3.77 & 4.25 \\
\hline Energy from fat (\%) & 9 & 10 & 25 \\
\hline Energy from protein (\%) & 15 & 15 & 13 \\
\hline Energy from carbohydrates (\%) & 76 & 75 & 61 \\
\hline Mineral mix AIN-93M (g\%) & 35 & 50 & 35 \\
\hline Vitamin mix AIN-93M (g\%) & 10 & 14 & 10 \\
\hline Choline bitartrate (g\%) & 2.5 & 3.5 & 2.5 \\
\hline
\end{tabular}




\section{SUPPLEMENTARY FIGURES}

A

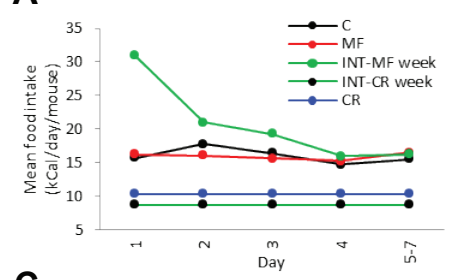

C
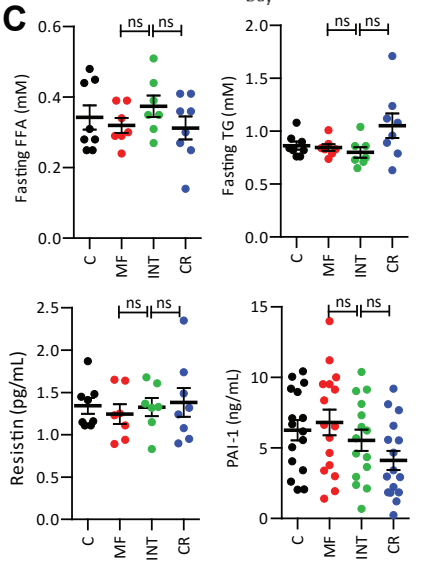

B

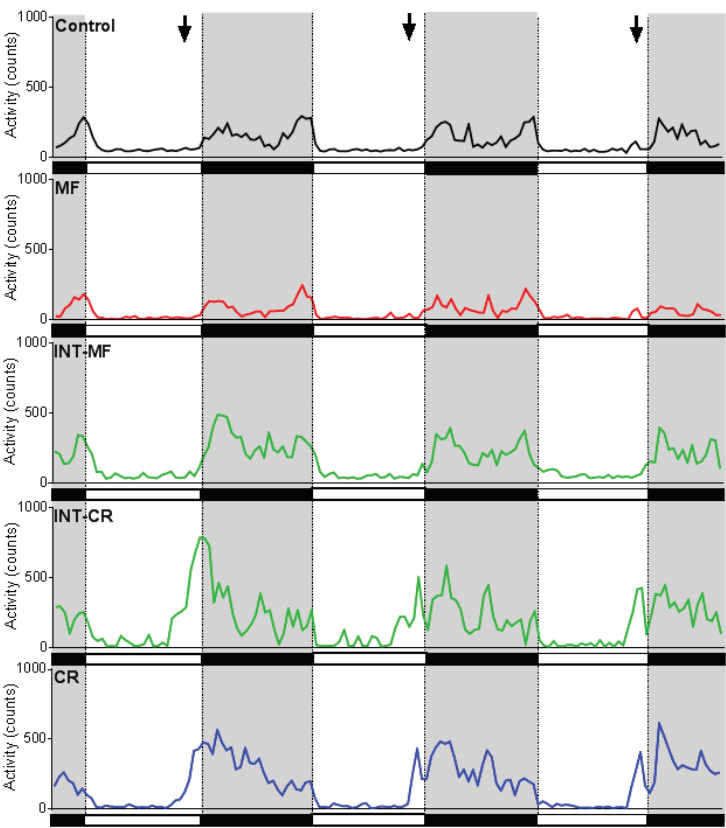

D

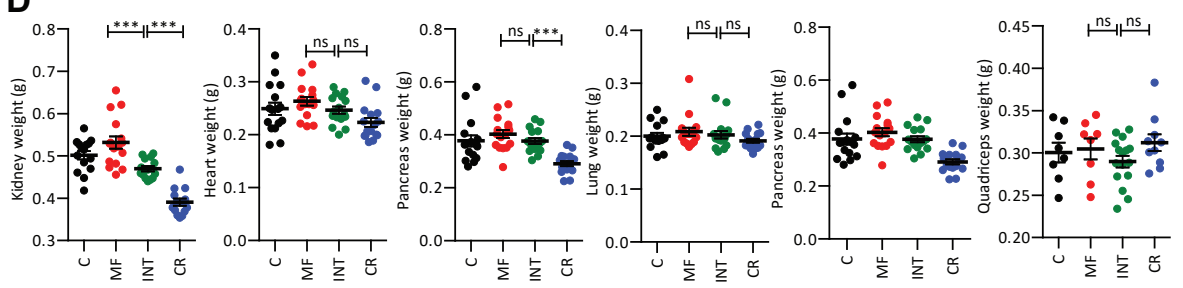

Supplemental Figure S1. Comparison of the phenotypic and biochemical features of the C, MF, INT and CR-exposed animals. (A) Food intake for \pm 20 mice per intervention group were measured during one week. For the INT and the MF/INT groups food intake during the ad libitum MF week and during 40E\% CR week are presented. (B) Actogram presenting the mean daily activity of mice from the different intervention groups recorded during 3 consecutive days. (C) Plasma insulin, Resistin and PAl-1 levels were measured using a Mouse Adipokine (MADKMAG-71K), according to the manufacturer's instructions. (D) Weight of the indicated organs was measured when the mice were sacrificed. 
A
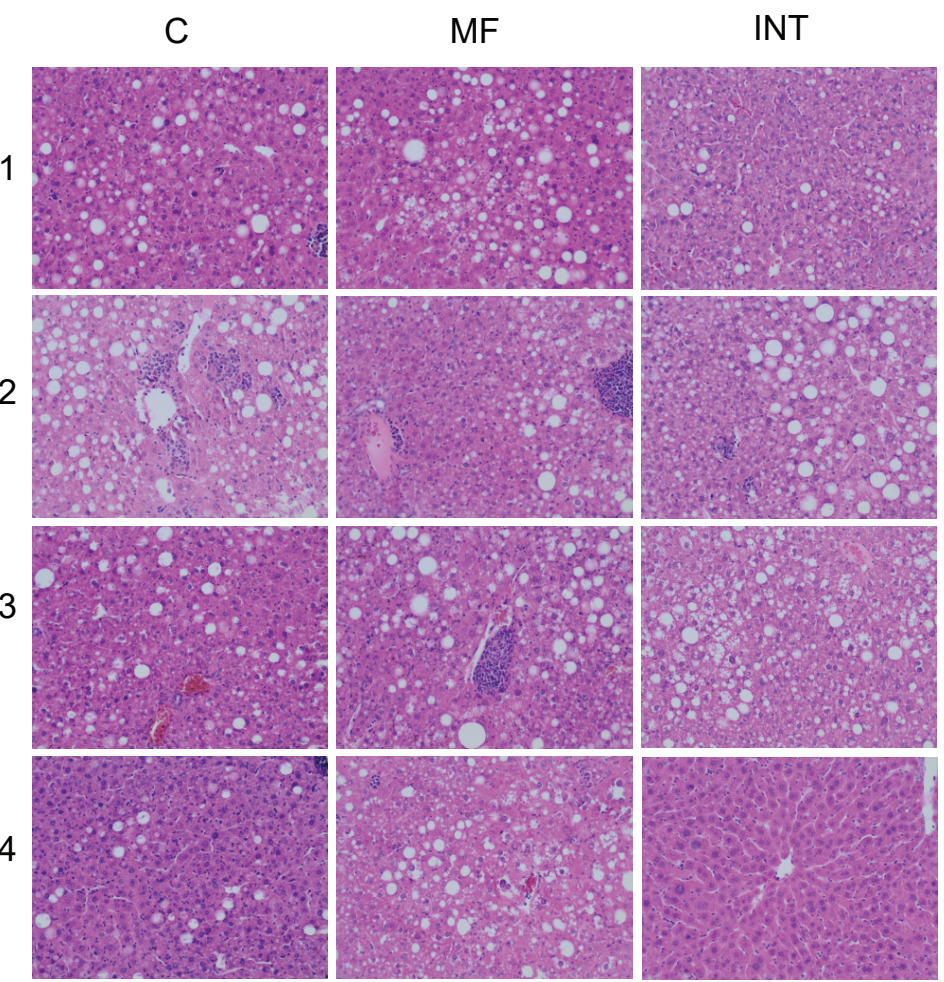

INT
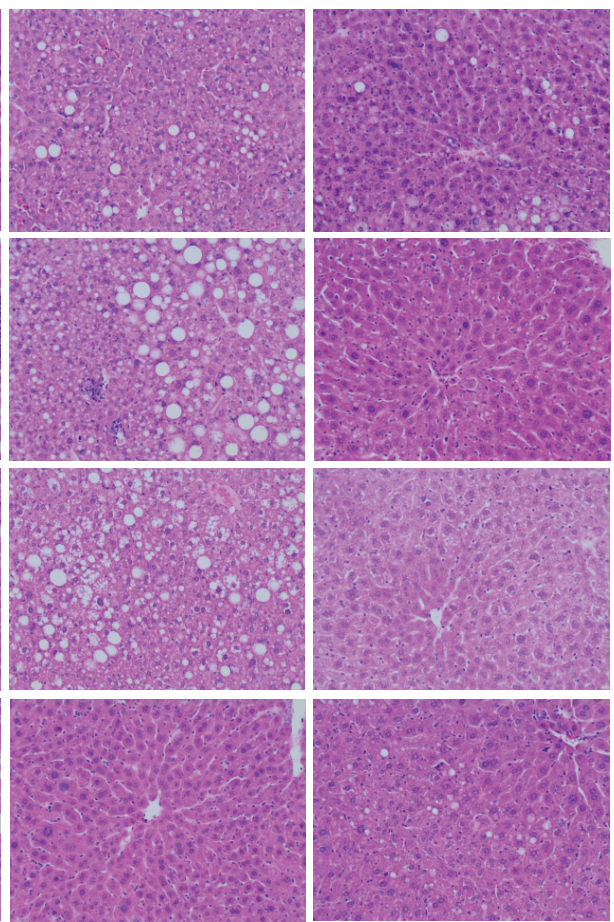

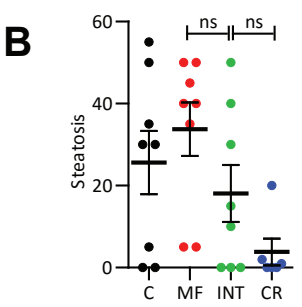

Supplemental Figure S2. Liver histology. (A) Formalin-fixed and paraffin-embedded cross-sections (5 $\mu \mathrm{m})$ of the liver lobe was stained with haematoxylin and eosin (H\&E). The results of 4 representative examples of the different intervention groups show hepatic steatosis in mice of the $C$ and MF groups and also, but to a lesser extent, in the INT-exposed mice. No lipid accumulation was detected in the life-long CR-exposed animals. (B) Samples were scored blindly by a board-certified pathologist using an adapted grading method for human NASH (Liang et al. 2014). Briefly, a $\mathrm{H} \& \mathrm{E}$ stained liver cross-section per mouse was examined and the level of steatosis was determined relative to the total liver area analysed (expressed as a percentage). 

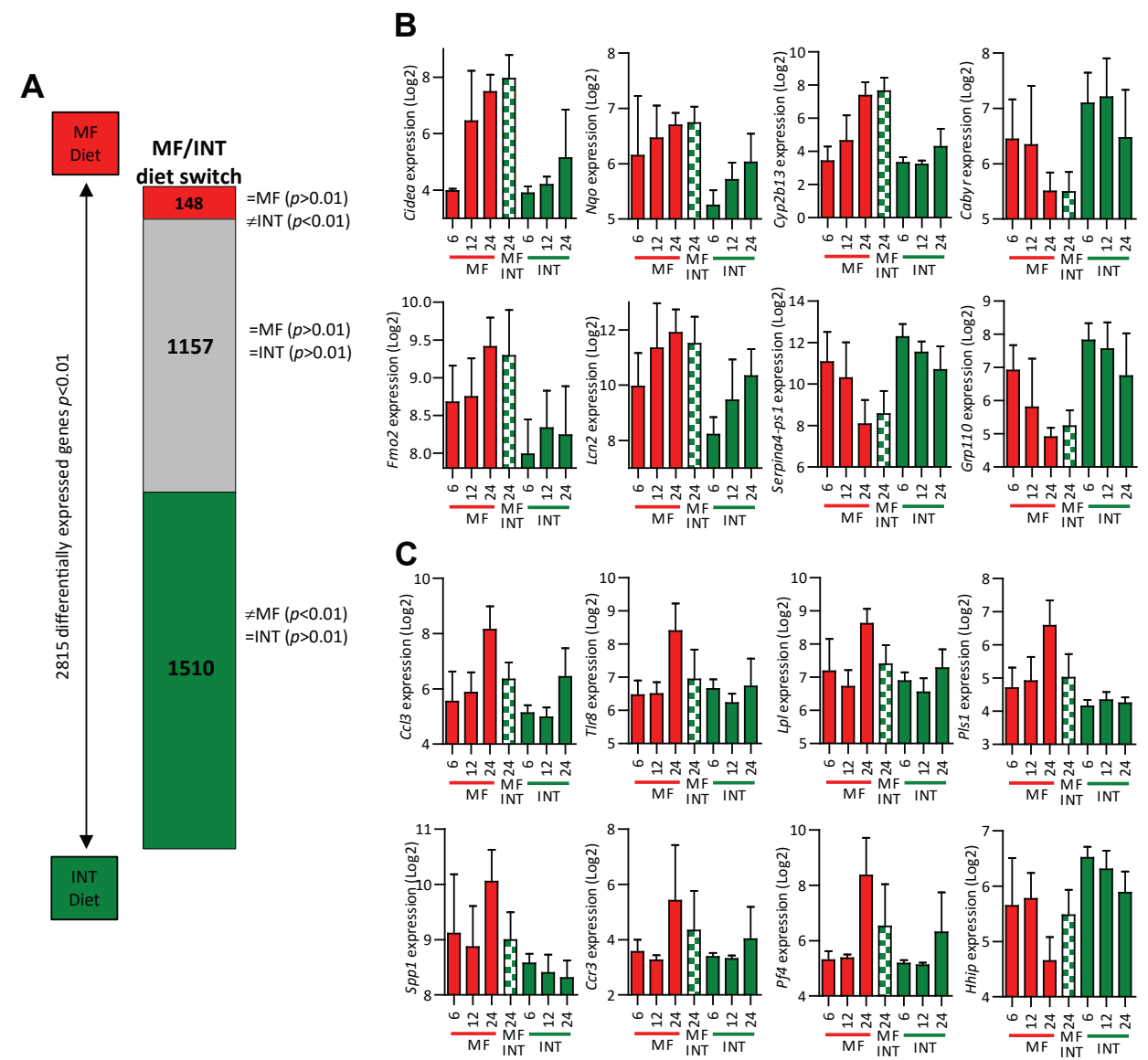

Supplemental Figure S3. (A) Schematic overview of the different gene groups revealing full (1510 genes) or partial (1157) adaptation to the INT diet and a subset of 148 genes of which the expression levels remained similar to the life-long exposed MF mice. (B) 8 examples of mice representing the 148 irreversible genes. (C) 8 examples of genes representing the 1510 adaptable genes. 



\section{ABSTRACT}

Calorie restriction (CR) is a dietary regimen that supports healthy aging. In this study we investigated the systemic and liver-specific responses caused by a diet switch to a medium-fat (MF) diet in 24-month-old life-long, CR-exposed mice. Nine-week-old C57BL/6J mice were exposed either to a control, CR or MF diet. At the age of 24 months, a subset of mice of the CR group was transferred to ad libitum MF feeding (CR-MF). The mice were sacrificed at the age of 28 months, then biochemical and molecular analyses were performed. Our results showed that, despite the longterm exposure to $\mathrm{CR}$ regimen, mice in the $\mathrm{CR}-\mathrm{MF}$ group displayed hyperphagia, rapid weight gain, and hepatic steatosis. However, no hepatic fibrosis/injury or alteration in CR-improved survival was observed in the diet switch group. The liver transcriptomic profile of CR-MF group largely shifted to a profile similar to the MF-fed animals but leaving $22 \%$ of the 1578 differentially regulated genes between the CR and MF diet groups comparable with the expression of the life-long CR group. Therefore, although the diet switch was performed at an old age, the CR-MF-exposed mice showed plasticity in coping with the challenge of a MF diet without developing severe liver pathologies. 


\section{INTRODUCTION}

Aging has been described as an important risk factor for most chronic diseases, largely due to the impaired capacity to maintain homeostasis and resilience against environmental stress or damage at old age. For the liver, aging has been associated with an increasing risk to develop nonalcoholic fatty disease (NAFLD) [1-5]. NALFD covers a spectrum of liver diseases ranging from simple steatosis to non-alcoholic steatozhepatitis (NASH), fibrosis and cirrhosis. While hepatic steatosis is considered to be benign, NASH is the more severe condition that is characterized by inflammation and possibly, fibrosis. Aging has been linked to NAFLD development through a number of commonly shared molecular mechanisms associated with both the NAFLD/NASH development and hallmarks of aging, e.g. reactive oxygen species formation, DNA damage and hepatocyte senescence [6, 7]. Aging and NAFLD are also intertwined with the modern obesogenic environment. The prevalence of obesity has been shown to increase at older age [8]. Commonly observed consequences of obesity are elevated plasma insulin and free fatty acid levels. Due to these changes, free fatty acid uptake and triglyceride production in the liver increase, leading to the development of NAFLD [9].

Calorie restriction (CR), a diet regimen of reduced energy intake without malnutrition, has been shown in numerous animal studies as by far the most effective approach to extend lifespan and to prevent age-related and metabolic diseases [10-12]. Research on CR in humans has not been conclusive, but provides clues that beneficial metabolic adaptations observed in model species also occur in humans $[13,14]$. The application of CR has been reported to be beneficial for liver health, by improving insulin sensitivity and reducing triglyceride accumulation in the liver [15-19].

The beneficial effects of $\mathrm{CR}$ are known to be induced immediately after the start of the application [20-23]. However, data concerning the response of long-term calorie-restricted subjects to ad libitum feeding, in particular the liver-specific responses at metabolic and transcriptomic level, are still limited. Among the few mice studies investigating the reversibility of CR-induced changes [20, 24-27], there are only two studies investigating the CR effects on liver physiology and transcriptome. Giller and co-workers showed that the effects of 6-month CR completely diminish within 2 weeks of control diet feeding in C57BL/6JRj mice [27]. Furthermore, Dhahbi and colleagues reported that in 34-month-old B6C3F1 mice exposed to a CR diet for 21 months, $90 \%$ of the CR-induced changes in gene expression in the liver disappear within 8 week of control diet feeding [20].

In the present study we exposed C57BL/6J mice to 30E\% CR from young (9 weeks) till old (24 month) age. Between 24 and 28 months of age we exposed a subset of mice in the CR group to an 
ad libitum medium-fat (MF) diet, thereby generating a CR-MF diet switch group. In the sacrificed 28-month old mice, we investigated the systemic and liver-specific responses to the diet switch at a physiological, metabolic and molecular level. In our study we explored both the plasticity of the mice at old age and the persistency of the effects caused by a life-long CR diet.

\section{RESULTS}

\section{Switching from a CR to MF diet at old age caused increasing body adiposity without affecting survival}

Nine-week-old C57BL/6J mice were randomly divided over 3 intervention groups and exposed to a control (C), calorie restriction (CR) or medium-fat (MF) diet. At the age of 24 months we replaced the diet of a subset of the CR intervention group by ad libitum exposure to the MF diet (CR-MF diet switch)

(Fig. 1A). All mice were sacrificed at the age of 28 months. Figure 1B shows that, at the time point of the diet switch, the body weights of the $\mathrm{C}$ and MF-exposed mice were substantially higher than that of the CR-exposed mice. Following the transfer to the MF diet, the body weight of the CR mice dramatically increased (Fig. 1B), reaching a new plateau at 27 months of age. At the moment of sacrifice, the body weight of the CR-MF diet switch group was significantly higher than that of the life-long CR-fed mice, comparable to that of the $\mathrm{C}$ group, but still significantly lower than that of the MF-exposed mice.

Food intake was recorded bi-monthly and revealed that, transferring mice from the CR intervention group to ad libitum MF feeding, resulted in severe hyperphagia. Food intake of the CR-MF group at the age of 26 months was even slightly higher than that of the life-long MF intervention group (Fig. 1C). However, at the age of 28 months, when the body weight gain was stabilized at a new plateau, food intake of the CR-MF mice decreased to similar amounts as consumed by the $\mathrm{C}$ intervention group.

Weight measurement of the epididymal white adipose tissue (eWAT) and liver revealed significant increases in the CR-MF group compared with the life-long CR-exposed mice (Fig. 1D). However, after normalization to body weight, a significant increase was observed in the CR-MF group only in relative eWAT weight, while no significant difference was found for the relative liver weight (Fig. 1E). In accordance with the eWAT weight, the plasma levels of leptin, one of the regulators of energy intake and fat storage secreted by adipose tissue, were found to be significantly higher in the CR-MF compared to the CR group (Fig. 1F). Correlation analysis revealed a significant positive correlation between eWAT weight and plasma leptin level (Fig. 1G; $r=0.7003, p<0.0001$ ).

Since $C R$ is known to protect against aging-related low grade systemic inflammation, which is also called inflammaging [28], a panel of 16 inflammatory markers, including interferon gamma 
(IFNץ), tumor necrosis factor (TNF), interleukin-1a (IL-1a ), IL-1ß, IL-2, IL-6, IL-7, IL-10, IL-15, chemokine (C-C motif) ligand 2 (CCL2 or MCP1), CCL3 (MIP-1a), CCL4 (MIP-1 $\beta$ ), CCL5 (RANTES), chemokine (C-X-C) motif ligand 1 (CXCL1 or KC), CXCL9 (MIG) and CXCL10 (IP-10), was measured in plasma to characterize the inflammation status following the exposure to MF diet. The PCA plot presented in Figure $\mathbf{1 H}$ revealed that the plasma inflammatory profile of the CR-MF switched animals had shifted into the direction of the $\mathrm{C}$-fed mice.

The survival rate recorded between 24 and 28 months revealed that mortality of the CR-MF diet switch group was equivalent to the life-long CR-exposed mice and strongly different from the $\mathrm{C}$ and MF intervention groups (Fig. 11). Therefore, despite the changes in whole body adiposity, the improved survival gained in the CR period was successfully maintained during the four months exposure to the MF diet.

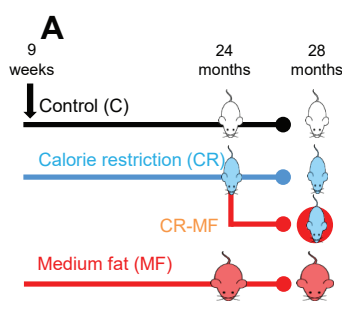

D

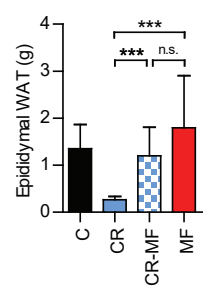

G

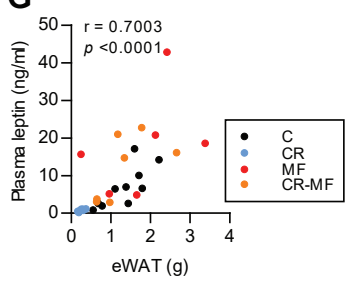

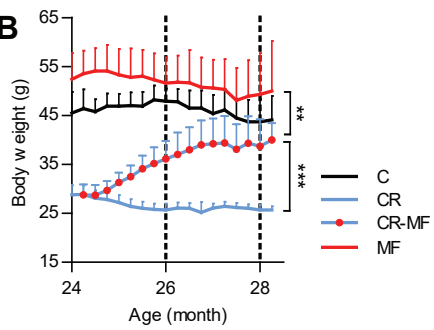

$\mathbf{E}$
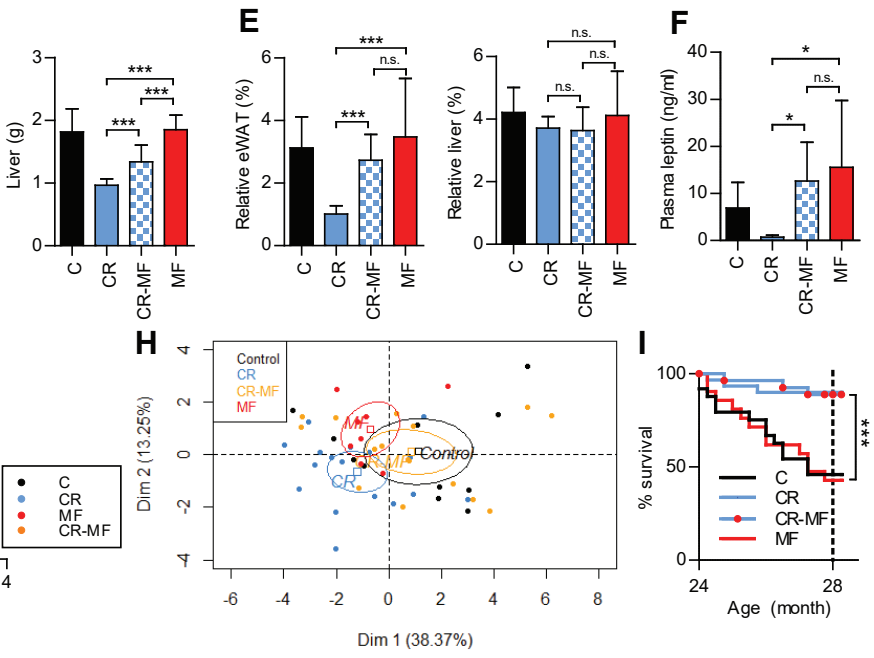

Figure 1. Physiological changes during the CR-MF diet switch. (A) Experimental design. (B) Body weight development from 24 to 28 month of age following the diet switch. (C) Food intake measurement at 24,26 and 28 month. (D) eWAT and liver weight. (E) Relative weight of eWAT and liver to the body weight. (F) Plasma leptin. Statistical significance was assessed by 1-way ANOVA followed by Tukey post-test analysis. (G) Correlation between eWAT weight and plasma leptin level, analyzed by applying Pearson's correlation. (H) PCA of 16 plasma inflammatory cytokines. (I) Kaplan-Meier survival curve, statistical difference was assessed by log rank analysis. Error bars reflect standard deviation (SD). ${ }^{*} p<0.05 ;{ }^{* *} p<0.01$; ${ }^{* *} p<0.001$. 


\section{The liver transcriptome of the CR-MF diet switch mice strongly shifted to the MF profile}

To investigate the diet switch effect on the liver transcriptome of the animals in the CR-MF diet group, a microarray analysis was performed. A principal component analysis (PCA) was carried out, using the $\mathrm{C}$ diet group as the reference. The results presented in Figure 2A show a remarkable shift in the transcriptome profile of the CR-MF diet switch group from the CR towards the MFexposed mice. Notably, the inter-individual variability in the MF diet group and in particular the CR-MF group, was much higher than in the CR group.

Although a pronounced switch in the transcriptomic profile of the CR-MF-exposed animals from the life-long CR group was detected, the diet switch did not result in a complete overlap with the expression profile of the MF-exposed group. To investigate which genes in the CR-MF expression profile remained similarly expressed with that of the life-long CR-exposed mice, we applied the following gene screening (see Fig. 2B). We first identified the differentially expressed genes between the life-long CR and MF groups $(p<0.01)$, which resulted in a list of 1578 genes. Then, to determine which of these 1578 genes remained comparable to the CR-exposed mice after the CR-MF switch and significantly different to those of the MF diet group, we screened for the genes 1) displaying no differential expression between the CR-MF and CR groups $(p \geq 0.05)$ and 2$)$ displaying significant $(p<0.01)$ difference in expression between the CR-MF and MF intervention groups. The direction of the fold change was also checked to confirm that the CR-MF and CR groups show the same direction of change in comparison to the MF group. A subset of 354 "CR- associated genes" were identified, exhibiting similar expression in the CR and CR-MF groups. Figure $\mathbf{2 C}$ visualizes the expression levels of the 354 CR-associated genes in the individual CR-, CR-MF- and MF-exposed mice. Overall, we showed that, following the CR-MF diet switch, 354 genes remained similarly expressed with the life-long CR exposed mice ( $22 \%$ of the 1578 genes), while the majority ( 78\% of the 1578 genes) adapted to the MF expression profile. 

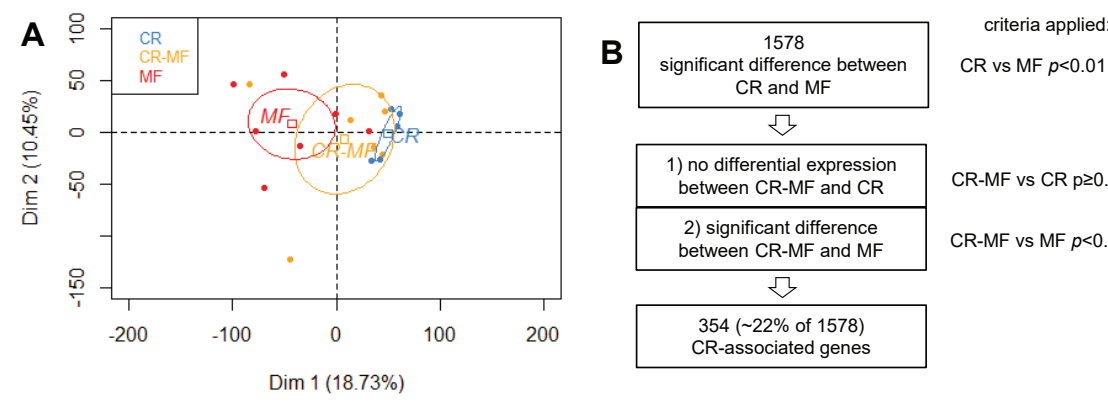

C
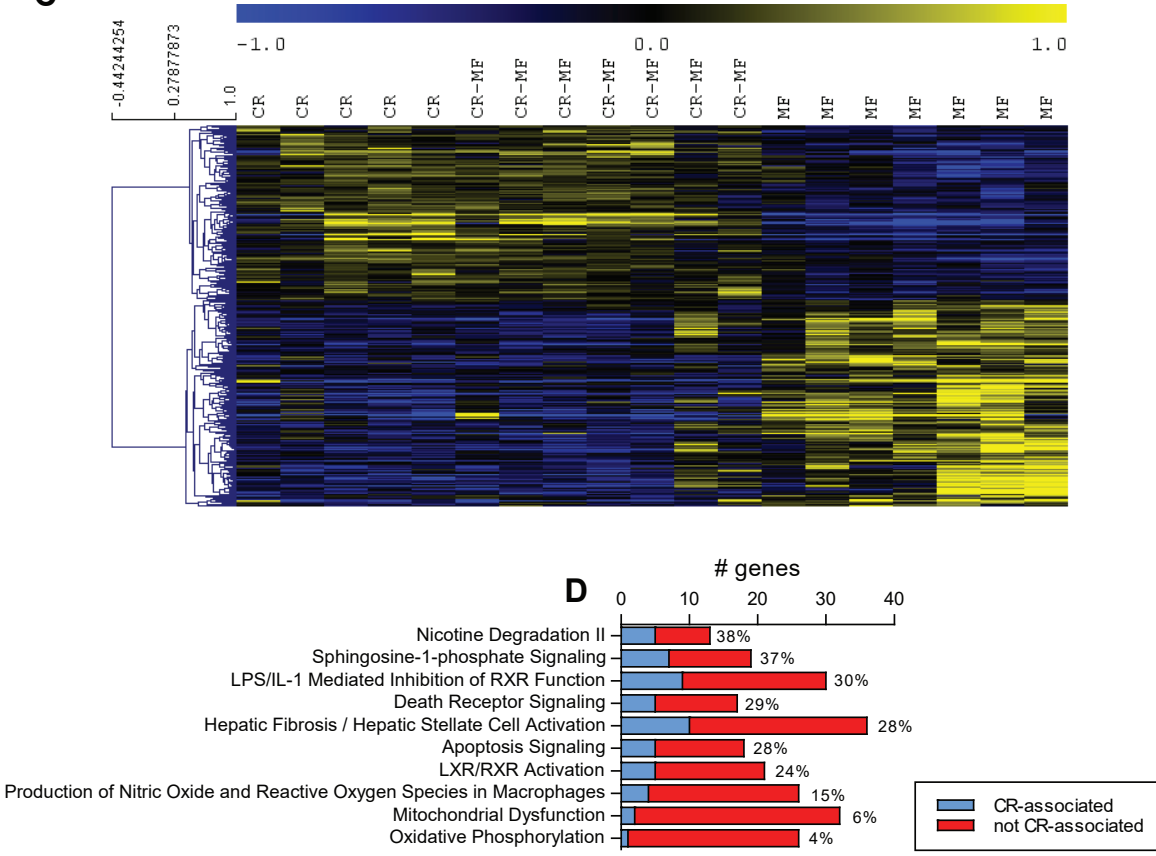

Figure 2. Liver transcriptomic profile of the CR-MF diet switch largely altered toward the direction of the life-long MF diet group. (A) PCA plot for individual animals showing the CR-MF animals shifted to the cluster of MF diet group. (B) Analysis scheme for investigating the status of the CR-differentially expressed genes after CR-MF diet switch. A smaller proportion of differentially expressed genes remained similar to the expression of the life-long $\mathrm{CR}$, while most of the genes shifted towards the profile of MF's. (C) Heatmap comparing the expression of $354 \mathrm{CR}$ associated genes in the CR, CR-MF and MF diet groups. (D) The fraction of the CR-associated and not CR-associated genes in the top 10 differentially regulated pathways between the life-long CR and MF diet groups.

\section{Functional characterization of the $\mathbf{C R}$-associated genes in the liver}

Ingenuity Pathway Analysis (IPA) was applied to explore which canonical pathways were represented by the 1578 genes displaying differential expression between the CR and MF intervention groups. The 10 most significantly different canonical pathways listed in Supplementary Table S1 revealed highly significant regulation of various pathways commonly acknowledged to be affected 
by $\mathrm{CR}$, including mitochondrial dysfunction, oxidative phosphorylation and apoptosis signaling. Next, we assigned the gene members in each pathway as a CR-associated or MF-adapted gene, according to the above explained criteria. The results presented in Figure 2D show that the pathways containing a relative high percentage of $\mathrm{CR}$-associated genes were found to be related to disease progression, e.g. sphingosine-1-phosphate signaling, apoptosis, hepatic fibrosis / hepatic stellate cell activation. Meanwhile, oxidative phosphorylation and mitochondrial dysfunction, both of which are pathways related to energy utilization, only contained a small fraction of the CRassociated genes (4\% and 6\%, respectively). This suggests that, following the CR-MF diet switch, pathways related to energy utilization largely adapted to the expression profile of the MF diet group. In addition, these results suggest that the CR-associated genes in the CR-MF diet switch group are not confined to a specific pathway. We extended the analysis to CR-regulated key metabolic pathways, which are known to be energy/nutrient sensing-dependent [29]. PCA performed on AMPK, PI3K/AKT and insulin-IGF (Supplementary Fig. S1) signaling's gene sets revealed that the expression profile of the CR-MF diet switch mice again strongly shifted from the CR profile toward the life-long MF cluster.

\section{Identification of upstream regulators of the CR-associated genes in the liver}

Next, by applying IPA, we searched for predicted upstream regulators of the 354 CR-associated genes. The results presented in Table 1 show the top 10 predicted up-stream regulators revealing highly significant $p$-values $\left(10^{-10}-10^{-3}\right)$. We observed that the regulators related to hepatic fibrosis including transforming growth factor $\beta 1$ (Tgf- $\beta 1$ ), interleukin-1 $\beta$ (IL1 $\beta$ ) and hypoxia-inducible factor 1-a (HIF-1a) were inhibited (activation z-score $<-2.000$ ). Two of these regulators, IL1 $\beta$ and HIF$1 \mathrm{a}$, are also related to inflammation. Furthermore, Acyl-CoA oxidase 1 (Acox1), which has been previously linked to NAFLD for its role in lipid metabolism [30], was predicted to be activated. Acox1 activity represents fat oxidation, which helps to prevent the accumulation of fat in the liver. 
Table 1. Upstream regulators of the $354 \mathrm{CR}$-associated genes

\begin{tabular}{lcccc}
$\begin{array}{c}\text { Top 10 upstream } \\
\text { regulator }\end{array}$ & $\begin{array}{c}\text { Target genes } \\
\text { (n) }\end{array}$ & $\begin{array}{c}\text { Predicted } \\
\text { activation } \\
\text { state }\end{array}$ & $\begin{array}{c}\text { Activation } \\
\text { z-score }\end{array}$ & p-value \\
ACOX1 & 17 & $\begin{array}{c}\text { Activated } \\
\text { TGFB1 }\end{array}$ & 3.153 & $1.32 \times 10^{-10}$ \\
AHR & 59 & Inhibited & -3.581 & $1.40 \times 10^{-9}$ \\
IL1B & 19 & Activated & 2.523 & $1.04 \times 10^{-6}$ \\
COMMD1 & 32 & Inhibited & -2.774 & $3.82 \times 10^{-6}$ \\
Alpha catenin & 5 & Activated & 2.236 & $1.56 \times 10^{-5}$ \\
BTNL2 & 10 & Activated & 2.618 & $1.94 \times 10^{-5}$ \\
HIF1A & 8 & Inhibited & -2.121 & $2.61 \times 10^{-5}$ \\
ERK & 16 & Inhibited & -2.140 & $2.04 \times 10^{-4}$ \\
CD44 & 11 & Inhibited & -2.121 & $9.71 \times 10^{-4}$ \\
\hline
\end{tabular}

\section{Development of hepatic steatosis, but no hepatic fibrosis/injury, in the CR-MF diet switch group}

Next we explored the CR-MF diet switch effect on NAFLD development in more detail. Measurement of plasma markers revealed that the plasma insulin levels increased in the CR-MF mice in response to the diet switch, but did not reach the levels of the life-long MF-exposed animals (Fig. 3A). The plasma alanine transaminase (ALT) level, a marker for liver injury, was markedly elevated only in the life-long MF diet group but not in any of the other intervention groups (Fig. 3B). The measurement of hepatic steatosis, which is represented by intrahepatic triglyceride (IHTG) content, revealed that after the diet switch the level of fat accumulation in the liver of the CR-MF diet group significantly increased to a level comparable to the life-long MF-exposed mice (Fig. 3C). The measurement of liver hydroxyproline content, a marker for hepatic fibrosis, showed elevated levels in the life-long MF-exposed animals, but not in the CR-MF group (Fig. 3D). To summarize, the 4 months exposure to MF diet resulted in elevated insulin and IHTG levels, but did not induce the progression of NALFD to liver fibrosis and injury. 

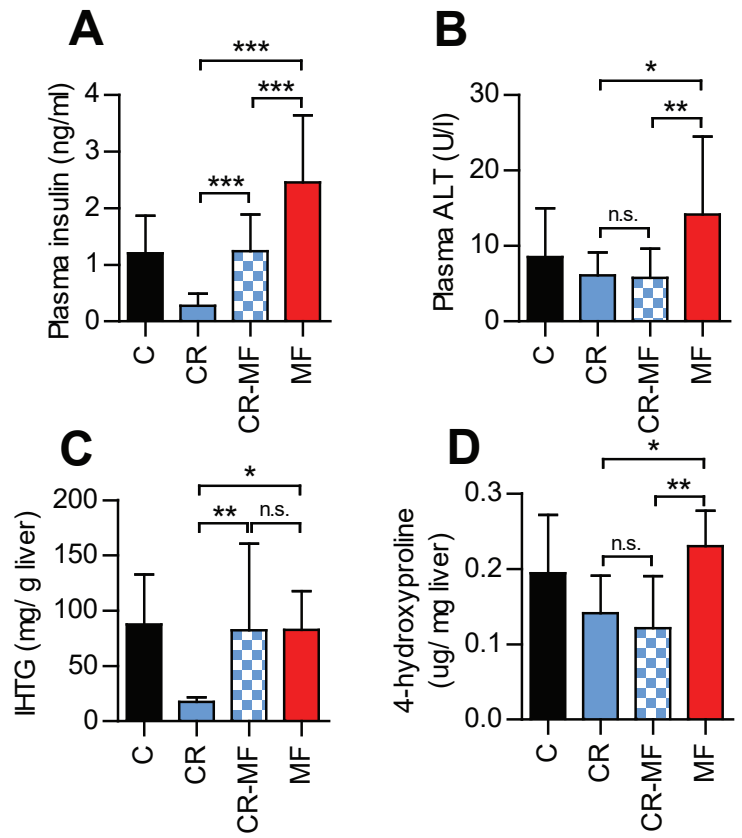

Figure 3. The CR-MF diet switch group demonstrated elevated insulin and IHTG levels, but comparable plasma ALT and liver hydroxyproline compared to the CR group. (A) Fasting plasma insulin level. (B) Plasma ALT. (C) Intrahepatic triglyceride (IHTG) content. (D) Liver hydroxyproline content. Statistical significance was assessed by 1-way ANOVA followed by Tukey post-test analysis. Error bars reflect SD. ${ }^{*} p<0.05$; ${ }^{* *} p<0.01$; ${ }^{* \star} p<0.001$.

\section{CR-MF diet switch shifted plasma $\mathbf{N}$-glycomics profile without altering the gene expression level of Fut8, one of the major glycosyltransferases}

Previous studies have shown that plasma N-glycosylation profiles are associated with chronic liver diseases [31]. The results presented in Figure 4A show that all three major N-glycan structures previously identified [32], bigalactosylated, biantennary glycan (NA2), agalactosylated, core-a-1,6-fucosylated biantennary glycan (NGA2F) and bigalactosylated, core-a-1,6-fucosylated biantennary glycan (NA2F), were significantly different between the life-long CR and MF diet groups. The levels of NA2, NGA2F and NA2F in the CR-MF diet group were in between those of the CR- and MF-fed animals, but did not differ significantly from either of the two intervention groups. Previous research has shown that expression and activity of a-1,6-fucosyltransferase (Fut8) in the liver was strongly associated with the plasma profiles of the three N-glycan structures (27). In our study, Fut8 expression differed significantly between CR and MF-exposed mice (Fig. 4B). However, Fut8 was one of the $354 \mathrm{CR}$-associated genes of which the expression did not alter in response to the diet 
switch. Interestingly, expression of another $\mathrm{N}$-glycan processing enzyme called ST8 a-N-acetylneuraminide a-2,8-sialyltransferase 4 (St8sia4) also differed significantly between CR and MFexposed mice, but expression of this gene was also affected in the CR-MF diet switch group (Fig. 4C). It can be speculated that the increased expression of other $\mathrm{N}$-glycan modifying factors, such as St8sia4 might be responsible for the increased glycosylation in the CR-MF diet switch group. Thus, despite that in the CR-MF group the hepatic expression of modifying enzyme Fut8 did not alter, a slight shift in the plasma $\mathrm{N}$-glycomics profile in the plasma was detected after the 4 months exposure to MF diet. St8sia4 might be responsible for the plasma glycosylation shift.
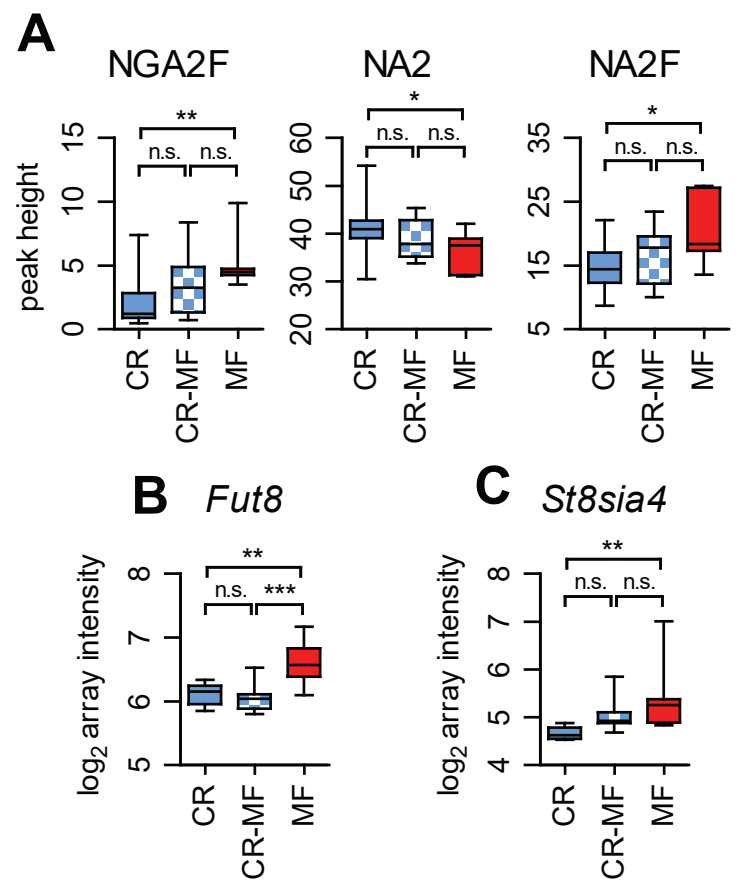

Figure 4. Plasma $\mathrm{N}$-glycosylation profile and expression levels of glycosylation modifying genes Fut8 and St8sia4. (A) Plasma levels of three N-glycans, NGA2F (peak 1), NA2 (peak 5), and NA2F (peak 6) had shifted following the CR-MF diet switch. Statistical significance was assessed by 1-way ANOVA followed by Tukey post-test analysis. (B) The expression levels of CR-associated gene Fut8 in the CR-MF diet switch group remained similar to those of CR and significantly differed from the expression levels of MF's. (C) Following the diet switch, gene expression levels of St8sia4 in the CR-MF group increased. Statistical difference for the gene expression data was determined by intensity-based moderated t-statistic (IBMT) $p$-value. Error bars denote 5 and 95 percentiles. ${ }^{*} p<0.05 ;{ }^{* *} p<0.01 ;{ }^{* * *} p<0.001$. 


\section{The discrepancy between physiological feature and related gene expression level following the CR-MF diet switch was also found for hepatic steatosis development and $C d 36$ expression}

In addition to the Fut8 liver expression and plasma glycosylation profile, the lipid metabolism gene expression in the CR-MF diet switch group also did not follow the anticipated patterns. While IHTG levels were significantly increased in the CR-MF diet switch group, cluster of differentiation 36 (Cd36), a key fatty acid transporter in the development of hepatic steatosis [33, 34], did not follow the same pattern. Cd36 displayed significantly different expression between the CR and MF intervention groups but its expression did not adapt to the MF diet after the diet switch (Fig. 5A). Also for this gene we could identify an alternative for fatty acid uptake, caveolin 1 (Cav1), which has been reported as contributors to fatty acid uptake [35]. Indeed, we found that the expression level of Cav1 was elevated in the CR-MF diet group (Fig. 5B), implying that, while the Cd36 expression was repressed, there was an alternative for fatty acid uptake.

\section{Differentially methylated enhancer region was found in the intergenic region adjacent to $\mathrm{Cd} 36$}

Since $C d 36$ plays an important role in the development of NAFLD, the mechanism behind its gene repression in the CR-MF diet switch is of biological interest. A possible mechanism underlying the $C d 36$ repression is by altering the DNA methylation of regions of the gene involved in transcription regulation. To explore this possibility, we analyzed the DNA methylation level of a promoter and enhancer region of $\mathrm{Cd} 36$. Promoter and weak enhancer regions were obtained from the mouse ChromHMM track [36, 37] and identified to be present in the gene body and a distant upstream region, respectively (Fig. 5C; for detailed chromosomal position refer to Supplementary Fig. S2). As shown in Figure 5D, we did not find a significant difference for the methylation level in the promoter region. However, the methylation levels of all $4 \mathrm{CpG}$ sites analyzed in the enhancer region were significantly higher in the MF diet group compared to the levels in CR group (Fig. 5E). Interestingly, although the methylation levels of CR-MF diet switch group were slightly increased compared to the life-long CR mice, the methylation percentage of each of the CpGs was markedly lower compared to the life-long MF-exposed animals. 


\section{A $\quad$ Cd36}

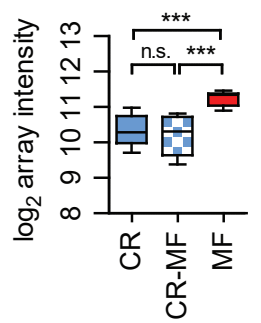

B Cav1

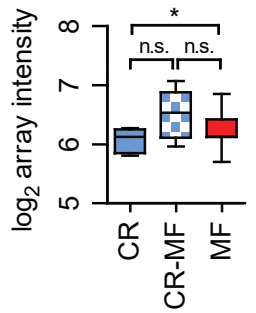

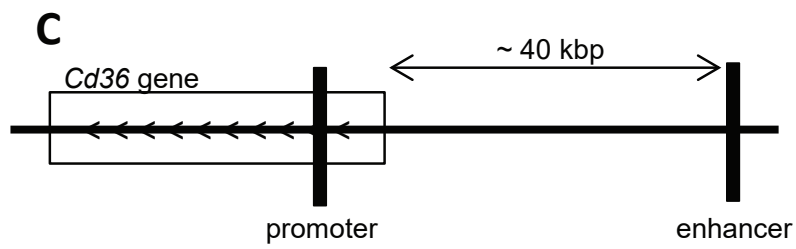

D

Promoter
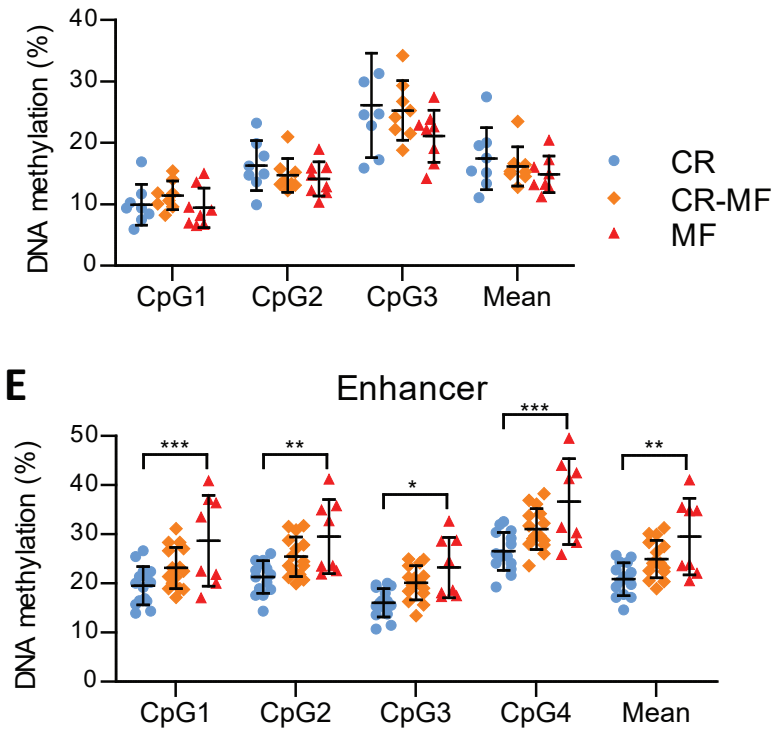

Figure 5. The gene expression levels of fatty acid uptake-related genes $\boldsymbol{C d} 36$ and $\mathrm{Cav} \mathbf{1}$, and DNA methylation levels of $\mathbf{C d 3 6}$. (A) The gene expression levels of CR-associated gene Cd36. (B) The gene expression levels of Cav1. Statistical significance of the gene expression data was determined by IBMT $p$-value. (C) The location of promoter and enhancer region upstream of $C d 36$. Error bars denote 5 and 95 percentiles. (D) DNA methylation levels of CR, CR-MF and MF in the promoter region. (E) DNA methylation levels in the intergenic enhancer region. Differences on methylation levels were analyzed by using two-way ANOVA followed by post-hoc Bonferroni test. Error bars represent SD. ${ }^{*} p<0.05 ;{ }^{* *} p<0.01 ;{ }^{* * *} p<0.001$. 


\section{DISCUSSION}

In this study we aimed to investigate the systemic and liver-specific responses of 24-monthold, life-long CR-exposed mice to 4 months of MF intervention. Our data revealed that in the diet switch group most of the CR-related features shifted to the MF profile: 1) whole body adiposity, 2) hepatic steatosis, 3) global transcriptome and 4) CR-specific molecular features including IGF$1 /$ insulin signaling, oxidative phosphorylation and AMPK signaling. These results show that the CR-MF-exposed animals have great plasticity in coping with the challenge of the MF diet. We also show that a number of CR-related features was maintained in the CR-MF group: 1) the prevention of hepatic fibrosis and injury, 2) the improved survival and 3) the expression levels of a subset of CR-related genes that were not altered by 4 months exposure to the MF diet.

Even after a long-term exposure to the $\mathrm{CR}$ diet until an old age of 24 months, the mice were not adapted to the low energy intake and displayed extreme hyperphagia. The life-long CR-exposed mice in our ageing cohort demonstrated their anticipation to receiving their daily food allotment by a burst in their activity level just prior to the regularly scheduled feeding [38]. The hyperphagic response is an indicator that hunger persists even after a long-term CR [39] and is maintained until body weight reaches the level of the ad libitum-fed animals [25]. Subsequently, hyperphagia is followed by a dramatic weight gain, adipose tissue expansion and hepatic steatosis. Metabolism has a thrifty "catch-up fat" characteristic, in which metabolic processes have evolved to be efficient in storing excessive energy once an energy supply is available [40]. Our experiment indicates that a life-long application of the $\mathrm{CR}$ regimen is not able to acclimatize the mice to low energy intake and to resist the thriftiness, when the food availability is no longer restricted.

Following the CR-MF diet switch, the beneficial effects of a CR diet on the liver transcriptomic profile changed dramatically, confirming the results of previous investigations [20,27]. This could be explained by the dependence of a number of CR-mediated pathways (e.g. oxidative phosphorylation, AMPK signaling) on energy depletion/stress [41, 42]. Despite the pronounced alteration of the transcriptomic profile, in our study we found a set of 354 CR-associated genes, which expression remained to be comparable with the expression levels of the life-long CR intervention group. This finding differs from the results of other studies [20,27]. Possible explanations for this difference include the experimental settings, such as the varying length of exposure to CR, severity of calorie reduction, diet type, mouse strain, and age at observation.

One of the most intriguing findings in the current study emerged when we investigated to what extent the CR-associated genes are linked to their related phenotypes. Previous studies on Fut8 hepatic expression and plasma glycosylation profile shows the modulation of Fut8 expression and 
NGA2F, NA2 and NA2F plasma levels during aging and chronic liver diseases [31, 32]. Expression of this gene did not alter during the 4 months exposure to MF diet, but a slight shift in the fucosylated $\mathrm{N}$-glycan levels, NGA2F and NA2F, in the plasma was observed. We hypothesized that St8sia4, which is also involved in $\mathrm{N}$-glycan processing, might be responsible for the increased glycosylation in the CR-MF diet switch group. Another possibility is the presence of other fucosyltransferases, such as Fut2 and Fut3 in the gut [43], which leads to plasma glycosylation profile modification by multiple tissues.

Another intriguing discrepancy in gene expression and physiological outcome is the hepatic expression of $\mathrm{Cd} 36$, a fatty acid transport gene the overexpression of which increases susceptibility to accumulate liver fat $[34,44]$. The deletion of this gene has previously been shown to cause resistance to diet-induced hepatic steatosis [45]. In our study we found that, despite of the development of hepatic steatosis, the expression of Cd36 in the CR-MF group remains low, comparable to the expression levels in the life-long CR diet group. The role of fatty acid transport was seemingly compensated by an increased expression of Cav1. This demonstrates that, after lifelong exposure to a CR diet, the system is still plastic and can adapt to a MF diet, but that alternative genes might be used when a certain function needs to be carried out.

Furthermore, we found that the methylation status of the far upstream enhancer ( $40 \mathrm{kbp}$ from the transcription start site), but not of the promoter region of the Cd36 was affected by the MF diet. Although this observation implies that changes in DNA methylation might be responsible for the altered expression levels of the $C d 36$ our results remain inconclusive. First of all, the methylation levels of the CR-MF diet switch group are in between those of the CR and MF groups, while the expression levels remain similar to those of the life-long CR-exposed mice. Secondly, in the MF intervention group both gene expression and methylation levels of the enhancer region of $C d 36$ were significantly increased, compared to the CR-fed mice. Decreased CD36 expression together with decreased DNA methylation has recently been shown in response to a hypocaloric diet-induced weight loss in female subjects [46]. Similar to what we observed, this study showed that gene expression and DNA methylation levels are positively associated, contrary to the current notion that increased methylation leads to transcription repression. However, the results presented in the study show differential methylation region close to the transcription start site, while in our study methylation of the enhancer was altered. Further studies are required to unravel the relation between gene expression and DNA methylation for the $C d 36$ gene.

To conclude, despite the long-term exposure to the CR regimen, the CR-MF diet switch group was not accustomed to this strict regimen and developed hyperphagia causing weight gain and hepatic steatosis. The liver transcriptomic profile of CR-MF group largely shifted to a profile similar 
to the MF-fed animals, leaving only $\sim 22 \%$ of the 1578 differentially regulated genes between the CR and MF diet groups comparable with the expression of the life-long CR group. As illustrated by the analysis of the expression of Cav1 and Cd36 expression and their relation with hepatic steatosis, the liver has a robust metabolic network that includes multiple regulators contributing its plasticity in coping with the challenge of MF diet. Therefore, although the diet switch was performed at an old age, the CR-MF-exposed mice showed plasticity in adapting to the MF diet_without developing severe liver pathologies, which likely contributes to the maintenance of the CR-improved survival. 


\section{MATERIAL AND METHODS}

\section{Ethics statement}

The institutional and national guidelines for the care and use of animals were followed and the Local Committee for Care and Use of Laboratory Animals at Wageningen University approved the experiment (code number: drs-2010151b).

\section{Animals and diets}

Male C57BL/6J mice were purchased from Janvier (Cedex, France) at 7 weeks of age and allowed to acclimate for 2 weeks, receiving standard AIN-93G (Research Diet Services, Wijk bij Duurstede, The Netherlands) upon arrival. In the light and temperature $\left(20^{\circ} \mathrm{C}\right)$-controlled animal facility of Wageningen University (12-hour light/dark cycle, light on at 04.00), they were housed in pairs during the acclimatization period, but were individually housed after the dietary intervention started.

At the start of the diet intervention the mice were 9 weeks old and randomly distributed into three intervention groups: 1) Control diet receiving AIN-93W diet ad libitum ( $n=89) ; 2$ ) CR diet receiving AIN-93W-CR in portions containing 70E\% of the mean energy intake of the group of the control mice were provided each day at 15.30 ( $n=117) ; 3$ ) medium fat diet (MF) receiving AIN-93W-MF ad libitum $(n=127)$. AIN-93W-CR contained increased concentration of vitamins and minerals content in order to feed these mice the same concentrations of micronutrients as the mice receiving AIN-93W diet and avoid malnutrition. Complete diet composition is listed in Supplementary Table S2 (Research Diet Services, Wijk bij Duurstede, The Netherlands). All mice were provided with ad libitum access to water. The long-term dietary invention was continued until a sacrifice at the age of 28 month, but in addition to the three diet groups, at 24 month the animals in the CR diet group were randomly assigned either to remain on the CR diet $(n=30)$ or undergo a diet switch to the MF diet $(n=32)$. This resulted in a group of 25-32 animals in each group at 24 month. Anticipating that the animals would not be used to ad libitum feeding after exposure to CR for a long term, the food intake was increased gradually, by addition of $10 \% \mathrm{E}$ per week. Therefore, it took 3 weeks for the animals to be allowed to have MF ad libitum. Body weight of all mice was recorded weekly. Food intake of 20 mice of each intervention group was measured every two months. Portion sizes of the mice on the CR were based on the mean food intake of the $\mathrm{C}$-exposed mice measured during a time span of 7 days.

At the age of 28 months, 8-11 mice of each intervention group were sacrificed between 14.00 and 17.00 on 3 consecutive weeks. Prior to sacrifice each mouse was first fasted for 4 hours after which they received an intragastric gavage of $0.5 \%$ carboxymethyl cellulose $(\mathrm{CMC})$, then fasted again for another 6 hours. For the CR and CR-MF groups, there were additional 9 and 8 animals, respectively, 
and they were treated with an addition of Wy-14643 substance in their $0.5 \% \mathrm{CMC}$ intragastric gavage. Since Wy-14643 treatment gives an effect on gene expression level, these animals were not included in the transcriptomic analysis, but they were included in the phenotypical measurements. After sedation with a mixture of isoflurane (1.5\%), nitrous oxide (70\%) and oxygen (30\%), blood samples were collected by cardiac puncture, then followed by neck dislocation. Weight of various organs was measured and subsequently snap-frozen and stored at $-80^{\circ} \mathrm{C}$ until further molecular/biochemical analysis.

\section{Hepatic triglyceride and hydroxyproline content determination}

Liver triglycerides were determined in 5\% liver homogenates prepared in buffer containing 250 mM sucrose, 1 mM EDTA, 10 mM Tris- $\mathrm{HCl}$ (pH 7.5), using the triglyceride Liquicolor Monoreagent (Instruchemie, Delfzijl, The Netherlands). Hydroxyproline analysis was performed as previously described in Hillebrandt et al. [47].

\section{Plasma measurement}

Plasma concentration of ALT was measured with commercially available kits from Instruchemie (Delfzijl, the Netherlands). Plasma insulin, IL-6 and CCL2 levels were measured using a Mouse Adipokine (MADKMAG-71K) kit, while plasma IFNy, TNF, IL-1a, IL-1ß, IL-2, IL-7, IL-10, IL-15, CCL3, CCL4, CCL5, CXCL1, CXCL9 and CXCL10 from Mouse Cytokine (MCYTMAG-13K) kit (Millipore, Billerica, MA, USA), according to the manufacturer's instructions.

\section{RNA isolation}

Total RNA was isolated using TRIzol reagent (Invitrogen, Breda, The Netherlands), according to manufacturer's instructions. The RNA was treated with DNAse and purified on columns using the RNeasy microkit (Qiagen, Venlo, The Netherlands). RNA concentration was measured on a NanoDrop ND-1000 UV-vis spectrophotometer (Isogen, Maarsen, The Netherlands) and RNA integrity was checked on an Agilent 2100 Bioanalyzer (Agilent Technologies, Amsterdam, The Netherlands) with 6000 Nano Chips, according to manufacturer's instructions. RNA was judged as suitable only if samples showed intact bands of $18 \mathrm{~S}$ and $28 \mathrm{~S}$ ribosomal RNA subunits, displayed no chromosomal peaks or RNA degradation products and had a RNA integrity number (RIN) above 8.0.

\section{Microarray hybridization}

Hybridization, washing and scanning of Affymetrix GeneChip Mouse Gene 1.1 ST arrays were performed according to standard Affymetrix protocols. Microarray analysis was performed in 
MADMAX, a pipeline for statistical analysis of microarray data [48]. Arrays were normalized using the Robust Multiarray Average method [49, 50]. Probe sets were defined according to Dai et al. [51]. In this method probes are assigned to unique gene identifiers, in this case Entrez IDs. The probes on the Gene 1.1 ST arrays represent 21,225 Entrez IDs. For the analysis, only genes having 1) an inter-quartile range of $>0.1$ and 2 ) an intensity value of $>20$ on at least five arrays were taken into account, which resulted in 15,417 genes in the dataset. Array data will be submitted to the Gene Expression Omnibus.

For the microarray data analysis, differentially expressed probe sets were identified by using linear models (library limma) and the intensity-based moderated t-statistic (IBMT) method was applied [52,53]. Resulting $\log _{2}$ intensities and $p$-values were used for further descriptive bioinformatic analysis of the data. Heatmap and PCA plots were constructed by using MultiExperiment Viewer version 4.8.1 [54] and factomineR package in R, respectively. Pathway analysis was performed in Ingenuity Pathway Analysis (IPA; Ingenuity ${ }^{\circledR}$ Systems).

\section{Plasma glycomics analysis}

$\mathrm{N}$-glycans on the plasma glycoproteins were analyzed by using DNA Sequencer-Aided, Fluorophore-Assisted Carbohydrate Electrophoresis (DSA-FACE) technology. The same DSA-FACE protocol used in $[55,56]$ for the analysis of the human plasma $\mathrm{N}$-glycome was applied for the mouse plasma. Shortly, $2 \mu \mathrm{l}$ of total plasma was incubated for 5 min at $95^{\circ} \mathrm{C}$ with $2 \mu \mathrm{l}$ of $5 \%$ SDS in $10 \mathrm{mM} \mathrm{NH}_{4} \mathrm{HCO}_{3}$. The $\mathrm{N}$-glycans on the plasma glycoproteins were enzymatically released with 33 Units of PNG-ase F (New England Biolabs, Ipswich, MA, USA) in $3 \mu$ of 3.33\% NP-40 and 10 mM $\mathrm{NH}_{4} \mathrm{HCO}_{3}, \mathrm{pH} 8.3$ and incubated for $3 \mathrm{~h}$ at $37^{\circ} \mathrm{C}$. The released $\mathrm{N}$-glycans were desialylated with 2 $\mathrm{mU}$ of Neuraminidase of Arthrobacter ureafaciens (Roche Diagnostics GmbH, Mannheim, Germany) in $5 \mathrm{mM} \mathrm{NH}_{4} \mathrm{Ac}, \mathrm{pH} 5$, incubating for $3 \mathrm{~h}$ at $37^{\circ} \mathrm{C}$. Subsequently, $2 \mu \mathrm{l}$ of the desialylated $\mathrm{N}$-glycans in $5 \mu \mathrm{l}$ of water were dried completely at $60^{\circ} \mathrm{C}$ for $1 \mathrm{~h}$. After that, $2 \mu \mathrm{l}$ of a labeling solution (1:1 mixture of $20 \mathrm{mM}$ of fluorophore 8-amino-1,3,6-pyrenetrisulfonic acid (APTS) (Life Technologies, Carlsbad, CA, USA) in $1.2 \mathrm{M}$ citric acid and $1 \mathrm{M} \mathrm{NaCNBH}_{3}$ in DMSO) were added per sample and incubated overnight at $37^{\circ} \mathrm{C}$. The reaction was stopped with $150 \mu \mathrm{l}$ of water to each well. This solution was diluted 1:2.5 in water and $2 \mu$ of this solution were added to $8 \mu \mathrm{l}$ of D-Formammide for the sequencing analysis in a DNA-sequencer ABI-PRISM 3730xl (Applied Biosystem, Foster City, CA, USA). The electopherogram of the mouse plasma $\mathrm{N}$-glycans profile contained several peaks that were structurally characterized by overlapping their migration positions with those previously measured in the human plasma profile. Five major glycan peaks had the same migration positions of the well-known human N-glycan structures (Supplementary Fig. S3), in particular, 
peak 1 (NGA2F, an agalactosylated core-a-1,6-fucosylated, biantennary N-glycan), peak 3 (NG1A2F, a mono-galactosylated core-a-1,6-fucosylated, biantennary N-glycan), peak 5 (NA2, a bigalactosylated, biantennary N-glycan), peak 6 (NA2F, a bigalactosylated, core-a-1,6-fucosylated biantennary N-glycan) and peak 9 (NA3, a triantennary, trigalactosylated N-glycan). The heights of these peaks, that represent the relative concentrations of the $\mathrm{N}$-glycan structures, were quantified and normalized to the total signal intensity by using Peak Scanner software (Applied Biosystem,Foster City, CA, USA).

\section{DNA isolation from the liver tissue}

Genomic DNA was isolated from the liver by using the classical proteinase $\mathrm{K}$ digestion and phenol:chloroform extraction. The DNA was treated with RNase and eluted in RNase and DNase free distilled water. DNA purity and quantity were checked spectrophotometrically with NanoDrop ND1000 (NanoDrop Technologies, Wilmington, USA) and fluorometrically with Qubit DNA (Invitrogen, Oregon, USA).

\section{Bisulfite conversion and DNA methylation analysis}

Bisulfite conversion and DNA methylation analysis by means of pyrosequencing were adapted from a previous study [57]. For each sample, $1000 \mathrm{ng}$ of genomic DNA was bisulfite-treated using the EZ-96 DNA Methylation ${ }^{\text {TM }}$ Kit (Zymo Research, Irvine, CA, USA) and eluted in $60 \mu$ of TE. DNA methylation analysis was performed using PyroMark ${ }^{\mathrm{TM}}$ pyrosequencing technology (Biotage $A B$, Uppsala, Sweden). Primers were designed using PyroMark software, and the sequences of the primers used are listed in Supplementary Table S3. The PCR reactions were performed in a total volume of $45 \mu \mathrm{l}$, and the volume of bisulfite-treated genomic DNA used was $4 \mu \mathrm{l}$. PyroMark PCR Master Mix and CoralLoad Concentrate were used according to the manufacturer's instructions, and $0.2 \mu \mathrm{M}$ of each primer (Qiagen, Venlo, The Netherlands) was used. The following thermal cycling conditions were applied: $15 \mathrm{~min}$ at $95^{\circ} \mathrm{C}$, followed by 45 cycles of $94^{\circ} \mathrm{C}$ for $30 \mathrm{~s}$, tempX (genespecific, see Supplementary Table S3) for $30 \mathrm{~s}$, and $72^{\circ} \mathrm{C}$ for $40 \mathrm{~s}$, followed by a final elongation step at $72^{\circ} \mathrm{C}$ for $10 \mathrm{~min}$. The PCR product ( $35 \mu \mathrm{l}$ ) was bound to Streptavidin Sepharose HP beads (GE Healthcare, Uppsala, Sweden) and purified and made single-stranded using the Pyrosequencing Vacuum Prep Tool according to the manufacturer's instructions (Qiagen, Venlo, The Netherlands). Sequencing primers (for sequences, see Supplementary Table S3) were annealed to the purified single-stranded PCR product, and pyrosequencing was performed using the Q24 Pyrosequencing System (Qiagen, Venlo, The Netherlands). CpG methylation was analyzed with the provided software. 


\section{Statistical analysis}

Statistical analysis, except for the microarray gene expression data, were analyzed with GraphPad Prism 5.04. Differences between dietary interventions were analyzed using 1-way ANOVA followed by Tukey post-test analysis. The alteration of DNA methylation levels in a specific region was evaluated with two-way ANOVA followed by Bonferroni post-test analysis. Results represented in bar graphs are shown as means \pm standard deviation. For results plotted in box-andwhisker plots, the box extends from $25^{\text {th }}$ to $75^{\text {th }}$ percentiles with a line at median value, while the whiskers denote 5 and 95 percentiles. Points below and above the whiskers are drawn as individual dots. Pearson's correlation was used to determine the relationship between variables. Statistical significance for the survival of groups was established by the log-rank analysis of Kaplan-Meier plots.

\section{ACKNOWLEDGEMENTS}

We thank Elmar W. Tobi for his suggestion for identifying potential regions showing DNA methylation alteration and introducing ChromHMM track to annotate this region.

\section{DISCLOSURE OF POTENTIAL CONFLICTS OF INTEREST}

Authors have declared no conflict of interest.

\section{GRANT SUPPORT}

This work was financially supported by European Union's Seventh Framework Programme (FP7/2007-2011) IDEAL-aging under grant agreement no. 259679. 


\section{REFERENCES}

1. Lee, J.Y., Kim, K.M., Lee, S.G., Yu, E., et al., Prevalence and risk factors of non-alcoholic fatty liver disease in potential living liver donors in Korea: A review of 589 consecutive liver biopsies in a single center. Journal of Hepatology, 2007, 47,239-244.

2. Amarapurkar, D., Kamani, P., Patel, N., Gupte, P., et al., Prevalence of non-alcoholic fatty liver disease: population based study. Ann. Hepatol., 2007, 6,161-163.

3. Frith, J., Day, C.P., Henderson, E., Burt, A.D., et al., Non-Alcoholic Fatty Liver Disease in Older People. Gerontology, 2009, 55,607-613.

4. Argo, C.K., Northup, P.G., Al-Osaimi, A.M.S., and Caldwell, S.H., Systematic review of risk factors for fibrosis progression in non-alcoholic steatohepatitis. Journal of Hepatology, 2009, 51,371-379.

5. Angulo, P., Keach, J.C., Batts, K.P., and Lindor, K.D., Independent predictors of liver fibrosis in patients with nonalcoholic steatohepatitis. Hepatology, 1999, 30,1356-1362.

6. Hoare, M., Das, T., and Alexander, G., Ageing, telomeres, senescence, and liver injury. Journal of Hepatology, 2010, 53,950-961.

7. López-Otín, C., Blasco, M.A., Partridge, L., Serrano, M., et al., The Hallmarks of Aging. Cell, 2013, 153,11941217.

8. Ford, E.S., Giles, W.H., and Dietz, W.H., Prevalence of the metabolic syndrome among us adults: Findings from the third national health and nutrition examination survey. JAMA, 2002, 287,356-359.

9. Karpe, F., Dickmann, J.R., and Frayn, K.N., Fatty Acids, Obesity, and Insulin Resistance: Time for a Reevaluation. Diabetes, 2011, 60,2441-2449.

10. Spindler, S.R., Caloric restriction: From soup to nuts. Ageing Research Reviews, 2010, 9,324-353.

11. de Cabo, R., Carmona-Gutierrez, D., Bernier, M., Hall, Michael N., et al., The Search for Antiaging Interventions: From Elixirs to Fasting Regimens. Cell, 2014, 157,1515-1526.

12. Fontana, L. and Partridge, L., Promoting Health and Longevity through Diet: From Model Organisms to Humans. Cell, 2015, 161,106-118.

13. Fontana, L., Partridge, L., and Longo, V.D., Extending Healthy Life Span-From Yeast to Humans. Science, 2010, 328,321-326.

14. Mercken, E.M., Crosby, S.D., Lamming, D.W., JeBailey, L., et al., Calorie restriction in humans inhibits the PI3K/AKT pathway and induces a younger transcription profile. Aging Cell, 2013, 12,645-651.

15. Kirk, E., Reeds, D.N., Finck, B.N., Mayurranjan, M.S., et al., Dietary Fat and Carbohydrates Differentially Alter Insulin Sensitivity During Caloric Restriction. Gastroenterology, 2009, 136,1552-1560.

16. Ryan, M.C., Itsiopoulos, C., Thodis, T., Ward, G., et al., The Mediterranean diet improves hepatic steatosis and insulin sensitivity in individuals with non-alcoholic fatty liver disease. Journal of Hepatology, 2013, 59,138-143.

17. Masuoka, H.C. and Chalasani, N., Nonalcoholic fatty liver disease: an emerging threat to obese and diabetic individuals. Ann. N.Y. Acad. Sci., 2013, 1281,106-122.

18. Eckard, C., Cole, R., Lockwood, J., Torres, D.M., et al., Prospective histopathologic evaluation of lifestyle modification in nonalcoholic fatty liver disease: a randomized trial. Therapeutic Advances in Gastroenterology, 2013, 6,249-259.

19. Thoma, C., Day, C.P., and Trenell, M.I., Lifestyle interventions for the treatment of non-alcoholic fatty liver disease in adults: A systematic review. Journal of Hepatology, 2012, 56,255-266.

20. Dhahbi, J.M., Kim, H.J., Mote, P.L., Beaver, R.J., et al., Temporal linkage between the phenotypic and genomic responses to caloric restriction. Proc Natl Acad Sci U S A, 2004, 101. 
21. Hempenstall, S., Picchio, L., Mitchell, S.E., Speakman, J.R., et al., The impact of acute caloric restriction on the metabolic phenotype in male C57BL/6 and DBA/2 mice. Mechanisms of Ageing and Development, 2010, 131,111-118.

22. Lane, M.A., Tilmont, E.M., De Angelis, H., Handy, A., et al., Short-term calorie restriction improves diseaserelated markers in older male rhesus monkeys (Macaca mulatta). Mechanisms of Ageing and Development, $2000,112,185-196$.

23. Robertson, L.T. and Mitchell, J.R., Benefits of short-term dietary restriction in mammals. Experimental Gerontology, 2013, 48,1043-1048.

24. Dhahbi, J.M., Tsuchiya, T., Kim, H.J., Mote, P.L., et al., Gene expression and physiologic responses of the heart to the initiation and withdrawal of caloric restriction. J Gerontol A Biol Sci Med Sci, 2006, 61.

25. Selman, $\mathrm{C}$. and Hempenstall, S., Evidence of a metabolic memory to early-life dietary restriction in male C57BL/6 mice. Longevity \& Healthspan, 2012, 1,1-11.

26. Forster, M.J., Sohal, B.H., and Sohal, R.S., Reversible effects of long-term caloric restriction on protein oxidative damage. J Gerontol A Biol Sci Med Sci, 2000, 55.

27. Giller, K., Huebbe, P., Hennig, S., Dose, J., et al., Beneficial effects of a 6-month dietary restriction are timedependently abolished within 2 weeks or 6 months of refeeding - genome-wide transcriptome analysis in mouse liver. Free Radical Biology and Medicine, 2013, 61,170-178.

28. Franceschi, C., BonafÈ, M., Valensin, S., Olivieri, F., et al., Inflamm-aging: An Evolutionary Perspective on Immunosenescence. Ann. N.Y. Acad. Sci., 2000, 908,244-254.

29. Anderson, R.M. and Weindruch, R., Metabolic reprogramming, caloric restriction and aging. Trends in Endocrinology \& Metabolism, 2010, 21,134-141.

30. Fan, C.Y., Pan, J., Usuda, N., Yeldandi, A.V., et al., Steatohepatitis, spontaneous peroxisome proliferation and liver tumors in mice lacking peroxisomal fatty acyl-Coa oxidase: Implications for peroxisome proliferatoractivated receptor a natural ligand metabolism. Journal of Biological Chemistry, 1998, 273,15639-15645.

31. Blomme, B., Van Steenkiste, C., Grassi, P., Haslam, S.M., et al., Alterations of serum protein N-glycosylation in two mouse models of chronic liver disease are hepatocyte and not B cell driven. American Journal of Physiology - Gastrointestinal and Liver Physiology, 2011, 300,G833-G842.

32. Vanhooren, V., Dewaele, S., Kuro-o, M., Taniguchi, N., et al., Alteration in N-glycomics during mouse aging: a role for FUT8. Aging Cell, 2011, 10,1056-1066.

33. Wilson, C.G., Tran, J.L., Erion, D.M., Vera, N.B., et al., Hepatocyte-specific disruption of CD36 attenuates fatty liver and improves insulin sensitivity in HFD fed mice. Endocrinology, 2016, 0,en.2015-1866.

34. Sheedfar, F., Sung, M.M., Aparicio-Vergara, M., Kloosterhuis, N.J., et al., Increased hepatic CD36 expression with age is associated with enhanced susceptibility to nonalcoholic fatty liver disease. Aging, 2014, 6,281-295.

35. Pohl, J., Ring, A., Herrmann, T., and Stremmel, W., New concepts of cellular fatty acid uptake: role of fatty acid transport proteins and of caveolae. Proceedings of the Nutrition Society, 2004, 63,259-262.

36. Ernst, J. and Kellis, M., ChromHMM: automating chromatin-state discovery and characterization. 2012, 9,215-216.

37. Yue, F., Cheng, Y., Breschi, A., Vierstra, J., et al., A comparative encyclopedia of DNA elements in the mouse genome. Nature, 2014, 515,355-364.

38. van Norren, K., Rusli, F., van Dijk, M., Lute, C., et al., Behavioural changes are a major contributing factor in the reduction of sarcopenia in caloric-restricted ageing mice. Journal of Cachexia, Sarcopenia and Muscle, $2015,6,253-268$. 
39. Hambly, C., Mercer, J.G., and Speakman, J.R., Hunger does not diminish over time in mice under protracted caloric restriction. Rejuvenation Res, 2007, 10.

40. Barnes, S.K. and Ozanne, S.E., Pathways linking the early environment to long-term health and lifespan. Prog Biophys Mol Biol, 2011, 106.

41. Burkewitz, K., Zhang, Y., and Mair, William B., AMPK at the Nexus of Energetics and Aging. Cell Metabolism, $2014,20,10-25$.

42. Rizza, W., Veronese, N., and Fontana, L., What are the roles of calorie restriction and diet quality in promoting healthy longevity? Ageing Research Reviews, 2014, 13,38-45.

43. Drake, P.M., Cho, W., Li, B., Prakobphol, A., et al., Sweetening the Pot: Adding Glycosylation to the Biomarker Discovery Equation. Clinical Chemistry, 2010, 56,223-236.

44. Koonen, D.P.Y., Jacobs, R.L., Febbraio, M., Young, M.E., et al., Increased Hepatic CD36 Expression Contributes to Dyslipidemia Associated With Diet-Induced Obesity. Diabetes, 2007, 56,2863-2871.

45. Clugston, R.D., Yuen, J.J., Hu, Y., Abumrad, N.A., et al., CD36-deficient mice are resistant to alcohol- and high-carbohydrate-induced hepatic steatosis. Journal of Lipid Research, 2014, 55,239-246.

46. do Amaral, C.L., Milagro, F.I., Curi, R., and Martínez, J.A., DNA methylation pattern in overweight women under an energy-restricted diet supplemented with fish oil. BioMed research international, 2014, 2014,675021

47. Hillebrandt, S., Wasmuth, H.E., Weiskirchen, R., Hellerbrand, C., et al., Complement factor 5 is a quantitative trait gene that modifies liver fibrogenesis in mice and humans. Nat Genet, 2005, 37,835-843.

48. Lin, K., Kools, H., de Groot, P.J., Gavai, A.K., et al., MADMAX - Management and analysis database for multiple omics experiments. Journal of integrative bioinformatics, 2011, 8,160.

49. Bolstad, B.M., Irizarry, R.A., Åstrand, M., and Speed, T.P., A comparison of normalization methods for high density oligonucleotide array data based on variance and bias. Bioinformatics, 2003, 19,185-193.

50. Irizarry, R.A., Bolstad, B.M., Collin, F., Cope, L.M., et al., Summaries of Affymetrix GeneChip probe level data. Nucleic Acids Research, 2003, 31,e15.

51. Dai, M., Wang, P., Boyd, A.D., Kostov, G., et al., Evolving gene/transcript definitions significantly alter the interpretation of GeneChip data. Nucleic Acids Research, 2005, 33,e175.

52. Ritchie, M.E., Phipson, B., Wu, D., Hu, Y.F., et al., limma powers differential expression analyses for RNAsequencing and microarray studies. Nucleic Acids Research, 2015, 43.

53. Sartor, M.A., Tomlinson, C.R., Wesselkamper, S.C., Sivaganesan, S., et al., Intensity-based hierarchical Bayes method improves testing for differentially expressed genes in microarray experiments. BMC Bioinformatics, 2006, 7,538.

54. Saeed, A.I., Bhagabati, N.K., Braisted, J.C., Liang, W., et al., TM4 microarray software suite. Methods Enzymol, 2006, 411.

55. Borelli, V., Vanhooren, V., Lonardi, E., Reiding, K.R., et al., Plasma N-Glycome Signature of Down Syndrome. Journal of Proteome Research, 2015, 14,4232-4245.

56. Vanhooren, V., Laroy, W., Libert, C., and Chen, C., N-Glycan profiling in the study of human aging. Biogerontology, 2008, 9,351-356.

57. Steegenga, W.T., Mischke, M., Lute, C., Boekschoten, M.V., et al., Sexually dimorphic characteristics of the small intestine and colon of prepubescent C57BL/6 mice. Biology of Sex Differences, 2014, 5,1-17. 


\section{SUPPLEMENTARY TABLES}

Supplementary Table S1.10 most significantly different canonical pathways between the life-long $\mathrm{CR}$ and MF intervention groups

\begin{tabular}{l|l} 
Top 10 canonical pathways CR vs MF & \multicolumn{1}{l}{$p$-value } \\
\hline Mitochondrial dysfunction & $9.56 \times 10^{-9}$ \\
Oxidative phosphorylation & $1.04 \times 10^{-8}$ \\
Apoptosis signaling & $3.25 \times 10^{-7}$ \\
Production of NO and ROS in macrophages & $2.75 \times 10^{-5}$ \\
IL-4 signalling & $3.12 \times 10^{-5}$ \\
MSP-RON signalling pathway & $3.72 \times 10^{-5}$ \\
Rac signalling & $5.82 \times 10^{-5}$ \\
TNFR1 signalling & $7.31 \times 10^{-5}$ \\
Sphingosine-1-phosphate signalling & $1.12 \times 10^{-4}$ \\
Tumoricidal function of hepatic natural killer cells & $1.14 \times 10^{-4}$
\end{tabular}

Supplementary Table S2. Composition of the experimental diet. The CR diet was adjusted for the vitamins and minerals amount to ensure a homologous intake between both groups.

\begin{tabular}{l|ccc}
\multicolumn{1}{c}{ AIN-93W } & AIN-93W-CR & AIN-93W-MF \\
\hline Energy (kcal/g) & 3.85 & 3.77 & 4.25 \\
Energy from fat (\%) & 9 & 10 & 25 \\
Energy from protein (\%) & 15 & 15 & 13 \\
Energy from carbohydrates (\%) & 76 & 75 & 61 \\
Mineral mix AIN-93M (g\%) & 35 & 50 & 35 \\
Vitamin mix AIN-93M (g\%) & 10 & 14 & 10 \\
Choline bitartrate (g\%) & 2.5 & 3.5 & 2.5
\end{tabular}

Supplementary Table S3. Pyrosequencing primers details of Cd36 promoter and enhancer region

\begin{tabular}{|c|c|c|c|c|}
\hline Gene & $\begin{array}{c}\text { Chromosomal } \\
\text { position }\end{array}$ & Primer sequence (5‘ to $3^{\prime}$ ) & $\begin{array}{l}\text { Annealing } \\
\text { temp }\left({ }^{\circ} \mathrm{C}\right)\end{array}$ & $\begin{array}{l}\text { Amplicon } \\
\text { size (bp) }\end{array}$ \\
\hline $\begin{array}{l}\text { Cd36 } \\
\text { promoter }\end{array}$ & $\begin{array}{l}\text { ch5:17341776- } \\
17341855\end{array}$ & $\begin{array}{l}\text { FP: AAGTTTATAAGGGTTTATTITGGTGAAG } \\
\text { RP: ACTCCAACATCTAAAATAACAATTACAAT } \\
\text { SP: GTITIATTTAAGTAAGTTAGAGG }\end{array}$ & 57.8 & 205 \\
\hline $\begin{array}{l}\text { Cd36 } \\
\text { enhancer }\end{array}$ & $\begin{array}{l}\text { ch5:17435129- } \\
17435227\end{array}$ & $\begin{array}{l}\text { FP: TTGTTGTGTTAGGGAAATATTAATGAT } \\
\text { RP: ATAACTCCAACACCAACCACAATAA } \\
\text { SP: AGGGTAGTTATTTIAGTTAAGTTAG }\end{array}$ & 58.6 & 265 \\
\hline
\end{tabular}




\section{SUPPLEMENTARY FIGURES}

A

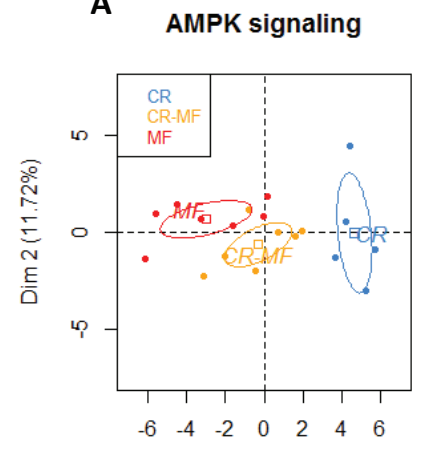

$\operatorname{Dim} 1(51.12 \%)$
B

PI3K/AKT signaling

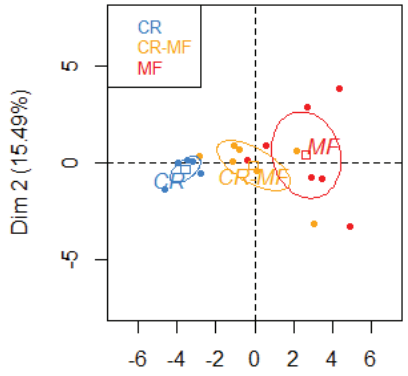

Dim 1 (50.02\%)
C insulin-IGF signaling

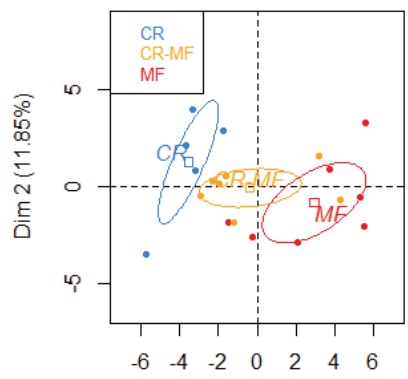

Dim 1 (32.82\%)

Supplementary Figure S1. PCA plots of gene sets revealing the shift of the gene expression profile following the CR-MF diet switch. (A) PCA of genes in the AMPK signalling. (B) PI3K/AKT signalling. (C) Insulin-IGF signalling. The expression values of all three dietary interventions were normalized to the $C$ group.

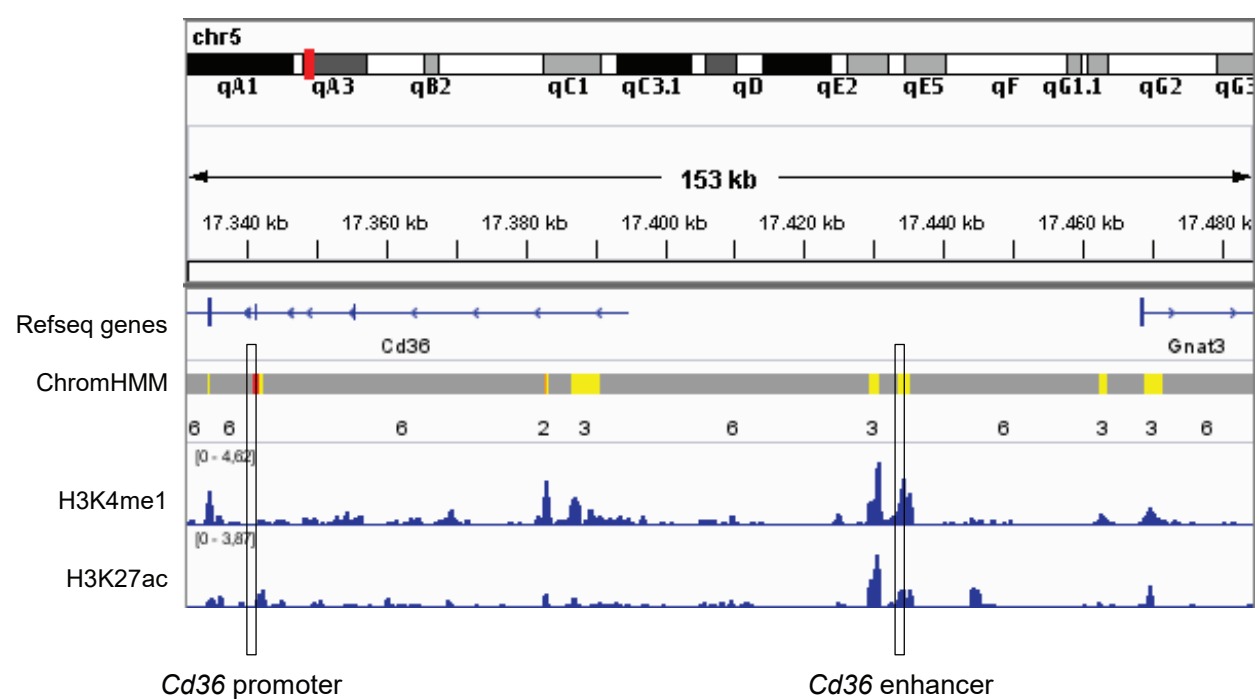

Supplementary Figure S2. Chromosomal position of regions analysed for differential DNA methylation level within and upstream of $\mathbf{C d} \mathbf{3 6}$ gene. Epigenetic features ChromHMM, H3K4me1 and H3K27ac were obtained from the mouse ENCODE database and visualized in integrative genomics viewer (IGV, Broad Institute). The red and yellow colors in ChromHMM track represent promoter and weak enhancer regions, respectively. The regions selected for DNA methylation analysis by pyrosequencing are marked with boxes. 


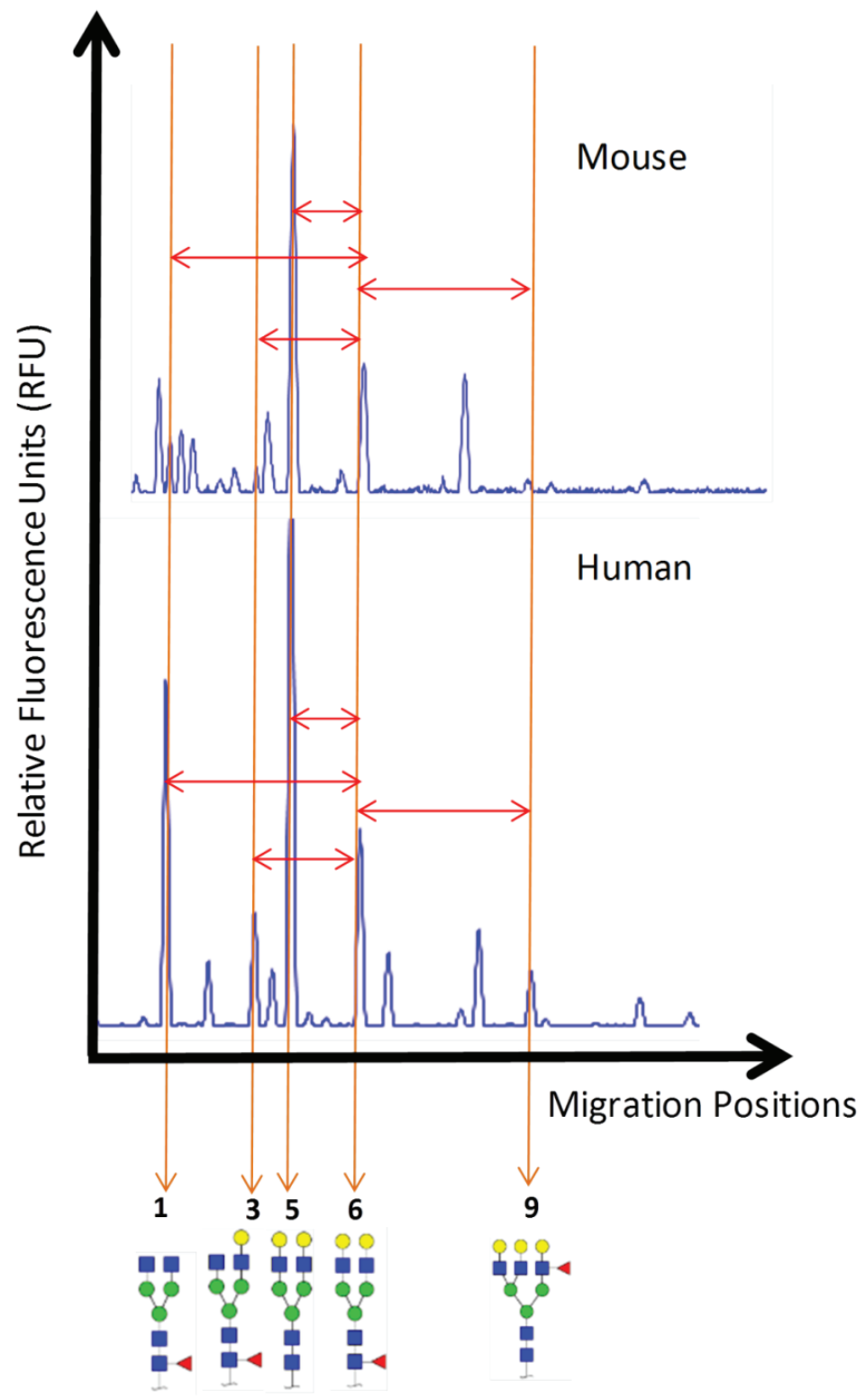

Supplementary Figure S3. Comparison of glycan migration position in mouse and human samples. Five major glycan peaks had the same migration positions of the well-known human N-glycan structures, i.e. peak 1 (NGA2F, an agalactosylated core-a-1,6-fucosylated, biantennary N-glycan), peak 3 (NG1A2F, a mono-galactosylated corea-1,6-fucosylated, biantennary N-glycan), peak 5 (NA2, a bigalactosylated, biantennary N-glycan), peak 6 (NA2F, a bigalactosylated, core-a-1,6-fucosylated biantennary $\mathrm{N}$-glycan) and peak 9 (NA3, a triantennary, trigalactosylated N-glycan). 



\section{ABSTRACT}

Fibroblast growth factor 21 (Fgf21) has emerged as a potential plasma marker to diagnose non-alcoholic fatty liver disease (NAFLD). To study the molecular processes underlying the association of plasma Fgf21 with NAFLD, we explored the liver transcriptome data of a mild NAFLD model of aging C57BL/6J mice at 12, 24, and 28 months of age. The plasma Fgf21 level significantly correlated with intrahepatic triglyceride content. At the molecular level, elevated plasma Fgf21 levels were associated with dysregulated metabolic and cancer-related pathways. The upregulated Fgf21 levels in NAFLD were implied to be a protective response against the NAFLDinduced adverse effects, e.g. lipotoxicity, oxidative stress and endoplasmic reticulum stress. An in vivo PPARa challenge demonstrated the dysregulation of PPARa signalling in the presence of NAFLD, which resulted in a stochastically increasing hepatic expression of Fgf21. Notably, elevated plasma Fgf21 was associated with declining expression of Klb, Fgf21's crucial co-receptor, which suggests a resistance to Fgf21. Therefore, although liver fat accumulation is a benign stage of NAFLD, the elevated plasma Fgf21 likely indicated vulnerability to metabolic stressors that may contribute towards progression to end-stage NAFLD. In conclusion, plasma levels of Fgf21 reflect liver fat accumulation and dysregulation of metabolic pathways in the liver. 


\section{INTRODUCTION}

Non-alcoholic fatty liver disease (NAFLD) has been recognised as a hepatic manifestation of metabolic syndrome. NAFLD covers a spectrum of liver injuries ranging from fat accumulation in the liver (steatosis) to the more severe condition of steatohepatitis (NASH). Considering that NAFLD is currently the most common liver disorder, prevalence of which has been reported to be $20-40 \%$ in the US [1, 2], the population would benefit from a diagnosis from the early stage of NAFLD. Intervention via as simple a method as weight management through diet and exercise is the most effective way leading to reduced liver fat, NASH remission, and also reduction of fibrosis [3]. However, at present a population-based screening tool for NAFLD is still lacking. Serum screening of liver enzymes and a liver ultrasound technique have been employed in screenings and clinical studies [4], but these procedures are, for different reasons, suboptimal. A number of studies have pointed out that the blood-screening test of the commonly analysed liver enzymes, including alanine aminotransferase (ALT), aspartate aminotransferase (AST), alkaline phosphatase (ALP), $\gamma$-glutamyl-transpeptidase $(\gamma-G T)$ and albumin, poorly diagnose NAFLD [5-8]. The ultrasound technique has better accuracy than the blood screening test, but is also suboptimal due to the low sensitivity of this method [9]. Magnetic resonance imaging and spectroscopy has a higher sensitivity, but this technique requires specific and expensive instruments, which limits its measurement availability. Diagnosis on liver biopsies is the most accurate way to determine the presence of NAFLD, but this procedure is highly invasive and not suitable for population-based screening. Hence, new accurate and non-invasive measures are required for the diagnosis of NAFLD.

Recently, fibroblast growth factor 21 (FGF21 (Fgf21 in mice)) has emerged as a potential diagnostic marker for NAFLD. Serum FGF21 is found to be elevated in NAFLD patients, as compared to healthy subjects, and correlates with hepatic fat content and the degree of liver steatosis $[4,10$ 12]. Moreover, the performance of FGF21 has been examined in a 3-year prospective study in China and high serum FGF21 was found to be a determinant of NAFLD, showing an area under curve of receiver operating characteristic (AUROC) of 0.816 [13]. Serum FGF21 has also been reported to increase the accuracy of non-alcoholic steatohepatitis (NASH) diagnosis using cytokeratin-18 fragment (CK-18) [14]. Therefore, plasma/serum FGF21 seems a promising diagnostic marker for an accurate and non-invasive diagnosis of NAFLD.

FGF21 has multiple metabolic functions, regulating energy homeostasis, glucose-lipid metabolism and insulin sensitivity [15]. However, it is currently unclear which metabolic functions of FGF21 underlie the association of plasma FGF21 level and NAFLD. In the present study, we aim to identify the putative molecular mechanisms that underlie the association of plasma FGF21 level with NAFLD. A complicating factor for a human study in this field is that the accurate assessment of 
NAFLD would require liver biopsies from healthy subjects, which is ethically undesirable. Therefore, we employed a cohort of aging mice to investigate the association between NAFLD, which was determined by IHTG level, and plasma Fgf21. The use of a cohort of aging mice, which consists of four different age time points, enables us to investigate whether plasma Fgf21 can act as a biomarker at different age time points. NAFLD has been reported in subjects of all ages, but particularly in middle to old age (40-65 years old in human) [16], which indicates that NAFLD develops over many years. To create a mice cohort that simulates the slow onset of NAFLD, we included a medium-fat diet (MF; 25E\% from fat) as a diet that induces the development of NAFLD. The energy contribution from fat in this diet group is considered mild, compared to other NAFLD-inducing dietary interventions that commonly acutely stimulate NAFLD pathologies within several weeks of feeding by applying a high-fat diet (45E\% from fat) $[17,18]$. In addition to the MF diet, we introduced a normal diet (10E\% from fat) as the control (C) group and a calorie restriction diet (CR; 30E\% reduced feeding compared to control group) as a diet regimen that prevents NAFLD. Then, we investigated whether the use of plasma Fgf21 as marker for NAFLD in our mice cohort is comparable to what has previously been reported for humans. We next searched for the biological processes underlying the association of plasma Fgf21 with NAFLD by performing microarray analysis on liver mRNA by using two approaches. First, we searched for pathways and upstream regulators associated with plasma Fgf21 by performing a gene co-expression network analysis using weighted gene co-expression network analysis (WGCNA) [19]. In the second approach, we investigated the major transcriptional difference in NAFLD by performing gene set enrichment analysis (GSEA), and then determining the relevance of Fgf21 in the NAFLD-related pathways. To further investigate PPARa signalling as one of the dysregulated pathways, we examined the PPARa response to its agonist at gene expression level. Since we found that some of the mice of 6 months of age displayed a high level of plasma Fgf21 in the absence of elevated IHTG levels, we also explored the microarray data to search for the functions of the genes associated with the elevated Fgf2 1 plasma level without accumulation of IHTG. 


\section{MATERIALS AND METHODS}

\section{Ethics statement}

The animal experiment was approved by the Local Committee for Care and Use of Laboratory Animals at Wageningen University (code number: drs-2010151b) and performed in accordance with the institutional and national guidelines for the care and use of animals.

\section{Mice aging study}

The mice aging study was a part of the IDEAL mice aging cohort that has been described in detail previously [20, 21]. Briefly, male C57BL/6 J mice (age of 7 weeks) were purchased from Janvier (Cedex, France) and were housed in pairs of two in the light and temperature $\left(20^{\circ} \mathrm{C}\right)$-controlled animal facility of Wageningen University (12-hour light/dark cycle, light on at 04.00). The mice were acclimated for 2 weeks, receiving standard AIN-93G (Research Diet Services, Wijk bij Duurstede, The Netherlands) upon arrival. All mice were provided with ad libitum access to water. The study design is presented in Supplementary Figure S1. The diet intervention started at the age of 9 weeks. The mice were housed individually and randomly distributed into three intervention groups: 1) control diet (C, 10E\% fat, $\mathrm{n}=89$ ) receiving AIN-93W diet ad libitum; 2 ) calorie restricted diet (CR, $\mathrm{n}=117$ ) receiving AIN-93W-CR in portions containing $70 \mathrm{E} \%$ of the mean energy intake compared to the mice on the control diet; 3 ) medium fat diet (MF, 25E\% fat, $\mathrm{n}=127$ ) receiving AIN-93W-MF ad libitum. AIN-93W-CR contains an increased concentration of vitamins and minerals in order to feed these mice the same concentrations of micronutrients as the mice receiving the AIN-93W diet and avoid malnutrition. Portion sizes for the mice on the CR were based on food intake of mice on the control diet and adjusted every 6 months. The rations were provided each day at 15.30, 30 minutes before the light was switched off. The complete diet compositions are listed in Supplementary Table S1 (Research Diet Services, Wijk bij Duurstede, The Netherlands).

The mice were culled at the age of 6, 12, 24 and 28 months. At each sacrifice, 12-18 mice of each intervention group were sacrificed between 14.00-17.00 on consecutive days (the remaining mice stayed in the experiment and were evaluated at older ages). Mice were paired per dietary intervention group according to body weight at sacrifice, so that mock and Wy-14,643 (Wy) treatment were provided to mice with similar body weight. Prior to sacrifice each mouse was first fasted for 4 hours, after which they received an intragastric gavage of either solvent $(0.5 \%$ carboxymethyl cellulose) or Wy dispersed in solvent (160 mg Wy/kg body weight) and were fasted again for another 6 hours. Body weight, liver weight, IHTG and 4-hydroxyproline were measured in both the mockand Wy-treated animals. Plasma Fgf21 and liver microarray analysis were performed for mock- 
treated animals only, as Wy substance is a PPARa agonist potentially affecting Fgf21 expression. At the sacrifice, the mice were sedated with a mixture of isoflurane $(1.5 \%)$ in nitrous oxide $(70 \%)$ and oxygen (30\%). Blood samples were collected by cardiac puncture, which was followed by neck dislocation. Weight of various organs was measured and, subsequently, organs/tissues were snapfrozen and stored at $-80^{\circ} \mathrm{C}$ until further molecular/biochemical analysis.

\section{Measurement of hepatic steatosis and fibrosis}

Intrahepatic triglyceride (IHTG) content was determined in $5 \%$ liver homogenates prepared in buffer containing $250 \mathrm{mM}$ sucrose, $1 \mathrm{mM}$ EDTA, $10 \mathrm{mM}$ Tris- $\mathrm{HCl}$ (pH 7.5), using the triglyceride Liquicolor Monoreagent (Instruchemie, Delfzijl, The Netherlands), according to manufacturer's instruction. The IHTG level was applied as the diagnosis standard of NAFLD using the $5 \%$ or $50 \mathrm{mg}$ TG per gram liver criterion from Kleiner's scoring [22]. Liver fibrosis is represented by 4-hydroxyproline content measurement in the liver. The 4-hydroxyproline analysis was performed as previously described in Hillebrandt et al. [23].

\section{Measurement of plasma Fgf21 level and other plasma markers}

The plasma Fgf21 concentration was determined using Rat/Mouse FGF-21 ELISA kits (Milipore, cat \#EZRMFGF21-26K), according to the manufacturer's instructions. Plasma insulin was measured using a Mouse Adipokine (MADKMAG-71K) kit (Millipore, Billerica, MA, USA), according to the manufacturer's instructions. The cytokeratin-18 plasma concentration was measured using Mouse Cytokeratin 18-M30 ELISA kit (Cusabio, Hubei, China).

\section{RNA isolation}

Total RNA was isolated using TRIzol reagent (Invitrogen, Breda, The Netherlands), according to the manufacturer's instructions. The RNA was treated with DNAse and purified on columns using the RNeasy microkit (Qiagen, Venlo, The Netherlands). RNA concentration was measured on a NanoDrop ND-1000 UV-vis spectrophotometer (Isogen, Maarsen, The Netherlands) and RNA integrity was checked on an Agilent 2100 Bioanalyzer (Agilent Technologies, Amsterdam, The Netherlands) with 6000 Nano Chips, according to the manufacturer's instructions. RNA was judged as suitable only if samples showed intact bands of $18 \mathrm{~S}$ and $28 \mathrm{~S}$ ribosomal RNA subunits, displayed no chromosomal peaks or RNA degradation products and had a RNA integrity number (RIN) above 8.0.

\section{Microarray hybridization}

Hybridization, washing and scanning of Affymetrix GeneChip Mouse Gene 1.1 ST arrays were 
performed according to standard Affymetrix protocols as described previously [20, 24]. Microarray analysis was performed in MADMAX, a pipeline for statistical analysis of microarray data [25]. Arrays were normalized using the Robust Multiarray Average method [26, 27]. Probe sets were defined according to Dai et al. [28]. In this method probes are assigned to unique gene identifiers, in this case Entrez IDs. The probes on the Gene 1.1 ST arrays represent 21,225 Entrez IDs. For the analysis, only genes having 1) an inter-quartile range of $>0.1$ and 2) an intensity value of $>20$ on at least five arrays were taken into account, which resulted in 15,885 genes in the dataset. Array data have been submitted to the Gene Expression Omnibus, with accession number GSE84495.

\section{CDNA synthesis and real-time quantitative PCR}

The microarray data was validated by real-time quantitative PCR (Q-PCR). For each individual sample, single-stranded complementary DNA was synthesized from $1 \mu \mathrm{g}$ of total RNA using the First Strand cDNA Synthesis kit (Thermo Scientific, Landsmeer, The Netherlands), following the supplier's protocol. Q-PCR was performed using SensiMix SYBR No-ROX kit (Bioline, Alphen aan de Rijn, The Netherlands) and a CFX384 thermal cycler (Bio-Rad, Veenendaal, The Netherlands). The following thermal cycling conditions were used: $2 \mathrm{~min}$ at $94^{\circ} \mathrm{C}$, followed by 40 cycles of $94^{\circ} \mathrm{C}$ for $15 \mathrm{~s}$ and $60^{\circ} \mathrm{C}$ for 45 s. PCR reactions to validate Fgf21 expression were performed in duplicate and all samples were normalized to $R p l p 0$ expression. Primer sequences were retrieved from the online PrimerBank database [29] and the sequences of the primers used are listed in Supplementary Table S2.

\section{Statistical analysis}

Data were analysed with GraphPad Prism 5.04. The data was expressed as mean \pm standard error mean. Comparison between two groups was performed using student t-test, whereas comparison between 3 or more groups was performed using ANOVA. Correlation between two parameters was presented as Pearson correlation coefficient $(r)$ and $p$-value. A $p$-value of $<0.05$ was considered significant. The receiver operating characteristic (ROC) curve analysis was carried out and the area under the ROC curves (AUROCs) were calculated to represent their performance to predict NAFLD. Optimal cut-off points were calculated for sensitivity and specificity reference (Youden Index).

\section{Hepatic transcriptomics data analysis}

For the microarray data analysis, differentially expressed probe sets were identified by using linear models (library limma) and the intensity-based moderated t-statistic (IBMT) method was applied [30, 31]. Resulting $\log _{2}$ intensities and $p$-values were used for further descriptive 
bioinformatic analysis of the data. Gene set enrichment analysis (GSEA; http://www.broad.mit.edu/ gsea/) was performed in MADMAX. Gene sets with a false discovery rate (FDR) q-value of $<0.01$ were considered significantly enriched.

Gene co-expression networks (modules) were constructed using the blockwiseModules $R$ function in Weighted Gene Co-expression Network Analysis (WGCNA) [19]. WGCNA uses a network distance coupled with hierarchical clustering and dynamic tree cutting to define modules as branches of a cluster tree. Gene modules, which summarize the main patterns of variation, are defined in an unbiased fashion and denoted by colors. The first principal component represents the summary of the module and is referred to as the module eigengene (ME). MEs were then related to plasma Fgf21 level and other NAFLD-related traits. This approach avoids the multiple testing from thousands of individual transcripts to only a number of modules. To explore the functional pathways and predicted upstream regulators of the gene modules, Ingenuity pathway analysis (IPA; Ingenuity ${ }^{\circledR}$ Systems) was used. 


\section{RESULTS}

\section{Control and medium-fat diet groups developed NAFLD at middle and old age}

The dietary interventions exerted pronounced effects on the mice's body and liver weight, as shown in Figure 1A and B, respectively. During aging, the MF-exposed animals gained the highest body and liver weight, while the $\mathrm{CR}$-fed animals were the leanest. The weight gain was accompanied by an increase in IHTG content (Fig. 1C). While the CR-fed animals only showed a modest increase over time, the C- and MF-fed animals displayed an elevated IHTG level starting at middle-age, i.e. at 12 months. Liver fibrosis, which was represented by the measurement of 4-hydroxyproline (4-HP) content in the liver (Fig. 1D), showed to increase at old age (24 and 28 month) in the MF diet group.

The IHTG level was applied as the diagnosis standard of hepatic steatosis using the $5 \%$ or $50 \mathrm{mg}$ TG per gram liver criterion from Kleiner's scoring [22]. The prevalence of hepatic steatosis development in the cohort is depicted in Figure 1E. At the age of 6 months, none of the mice in any of the diet groups developed hepatic steatosis, indicating a healthy liver condition at young/mature adult age. Hepatic steatosis development became visible in 12-month old mice exposed to $C$ and MF diets, reaching up to $85.7 \%$ in the MF diet group. At the 24 -month time point, the prevalence of hepatic steatosis increased considerably in the control group. At this age over $80 \%$ in the $C$ and MF diet groups displayed steatosis (Fig. 1E) but at the age of 28 months, a slight decrease of prevalence was observed.

Since a criterion of 4-HP level for hepatic fibrosis diagnosis has not been clearly defined, we adapted the level of 4-HP that was reported by Fuchs et al. to be associated with extensive portal fibrosis (equivalent with Ishak fibrosis scoring stage 2-3) [32]. A level of $>0.200 \mu \mathrm{g}$ of 4 -HP per $\mathrm{mg}$ liver was applied as an indication of liver fibrosis. Increasing prevalence of hepatic fibrosis was only pronounced in the MF intervention group at 24 and 28 months of age (Fig. 1F). These results showed that hepatic steatosis occurred in both the $\mathrm{C}$ and MF intervention groups at the middle-age time point, while hepatic fibrosis in particularly developed in the MF diet group at an old age. On the other hand, the CR-fed animals were protected from developing hepatic steatosis and fibrosis. 
A
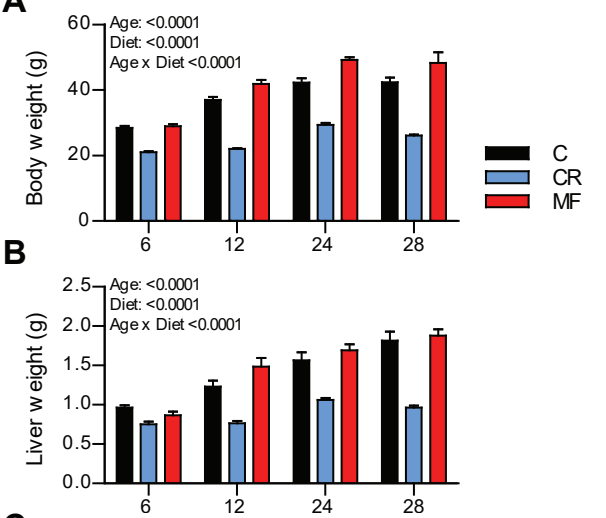

D $\quad 0.3$ Age: $<0.0001$

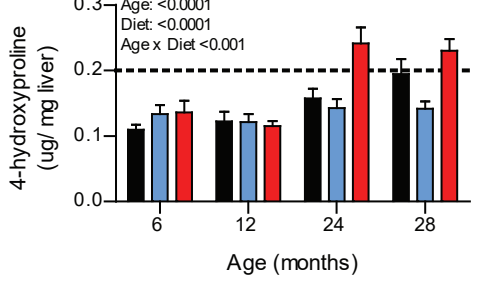

C
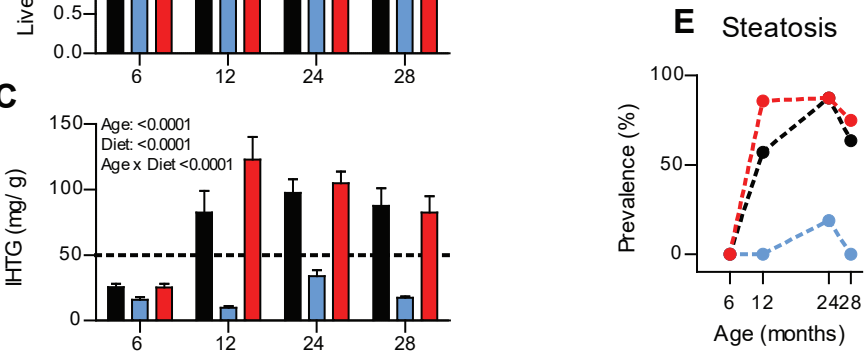

F Fibrosis

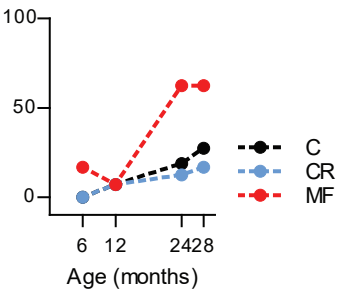

Figure 1. Physiological changes induced by the different dietary interventions at the age of $6,12,24$, and 28 months. Body weight (A), liver weight (B) and IHTG content (C) dramatically increased over time, except for the CR-fed animals which stayed lean. Significance ( $p$-value) of age, diet and interaction were evaluated using two-way ANOVA. Error bars represent s.e.m. Prevalence of hepatic steatosis $(E)$ and fibrosis $(F)$ in the mice aging cohort at different age time points.

\section{Plasma Fgf21 reflected the hepatic fat accumulation at middle and old age, but not at mature adult age}

The results presented in Figure 2A show that plasma Fgf21 levels of the C- and MF-exposed animals were higher than that of the CR-exposed animals at all ages. Noticeably, plasma Fgf21 levels were particularly high at 6 month of age in the $C$ and $C R$ diet groups. Next, to examine a specific diet- or age-related effect on plasma Fgf21 levels, the correlation with IHTG content was carried out separately by diet and age. The CR diet group displayed low IHTG and plasma Fgf21 concentration, while the C and MF groups showed some variation, but overall we did not observe significant correlation in any of the different diet groups (Fig. 2B). On the other hand, the comparison by age indicated that, except for the 6-month-old mice, the correlations at different ages were comparable (Fig. 2C). Some of the young mice, independent of their diet types, exhibited high plasma Fgf2 1 concentrations despite their low IHTG levels. This observation implies that the plasma Fgf21 concentration reflects the hepatic fat accumulation at middle-age ( 12 months) and old age (24 and 28 months), which results in an overall correlation coefficient of $0.52(p<0.0001)$, but performs differently at younger age (Fig. 2D). 


\section{Plasma Fgf21 levels reflected liver Fgf21 expression}

The gene expression levels of Fgf21 in the liver were obtained from the microarray data and revealed that, similar to the plasma Fgf21 levels, the $\mathrm{C}$ - and MF-exposed animals displayed higher expression levels of Fgf21 (Fig. 3A). Notably, the higher levels of plasma Fgf21 at the earliest time point of 6 months were also reflected in the Fgf21 liver expression level. The results presented in Figure 3B show that the plasma Fgf21 levels significantly correlated to hepatic Fgf21 expression levels obtained by microarray analysis $(r=0.63, p<0.0001)$. This result was confirmed by Q-PCR analysis ( $r=0.71, p<0.0001$, Supplementary Fig. S2A). These observations indicate that the plasma Fgf21 concentration reflects the Fgf21 mRNA levels in liver tissue. In addition, we examined whether plasma Fgf21 increased with body or liver weight. Correlation analyses confirmed that plasma Fgf21 had significant positive correlation with body and liver weights (Supplementary Fig. S2B and C). As Fgf21 has been reported to be expressed abundantly, not only in the liver, but also in other tissue types, we also examined whether the plasma Fgf21 concentrations reflect the Fgf21 expression levels in epidydimal white adipose tissue (eWAT), tibialis anterior muscle and colon tissue. The results presented in Figure $\mathbf{3 C}, \mathbf{D}$, and $\mathbf{E}$ show that plasma Fgf21 levels was strongly associated with the liver expression, compared to the other tissues. The expression levels of $F g f 21$ in the muscle and colon were extremely low (Fig. 3D and E). The correlation of plasma Fgf21 and expression in eWAT was significant, however, the levels of expression in the eWAT were much lower compared to those in the liver tissue (Fig. 3C).

To examine whether plasma Fgf21 in mice indicates hepatic fat accumulation as observed in humans, the mice were divided into two groups: those that either developed or did not develop NAFLD according to the IHTG criterion of higher or lower than $50 \mathrm{mg}$ TG per gram liver from Kleiner's scoring [22]. This resulted in 53 animals without NAFLD, which largely consisted of young or CR-fed animals, and 36 animals with NAFLD, which mostly consisted of older animals or under the Cor MF dietary regimen. The average IHTG contents of the animals with and without NAFLD were 111.1 and $22.2 \mathrm{mg}$ TG per gram liver, respectively. The characteristics of animals with and without NAFLD are summarized in Supplementary Table S3, showing that the animals with NAFLD had a significantly heavier body weight, larger epididymal fat depot, enlarged liver, and lower liver 4-HP content. The levels of fasting plasma insulin and the liver injury marker alanine aminotransferase (ALT) were elevated in the group of animals with NAFLD. 

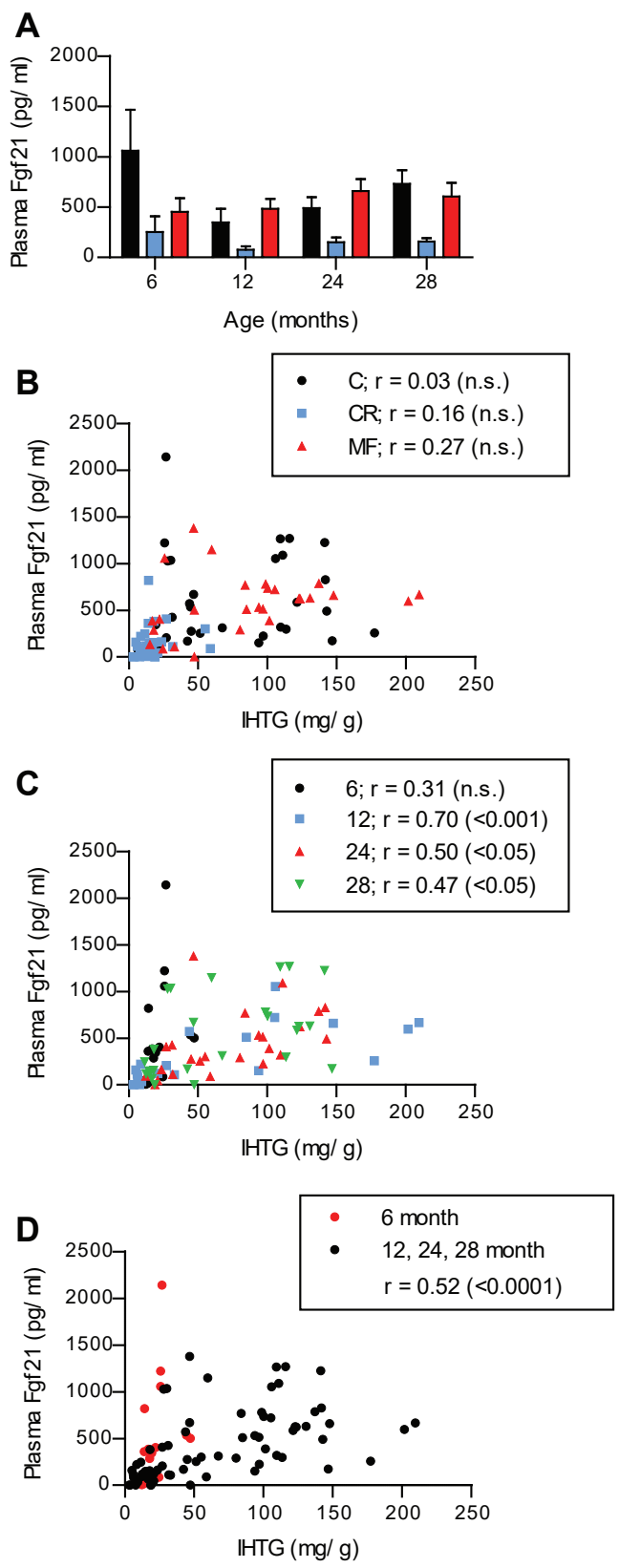

Figure 2. Plasma Fgf21 reflected the hepatic fat accumulation at middle and old age, but not at mature adult age. (A) Different plasma Fgf21 levels induced by the dietary interventions at the age of 6, 12, 24, and 28 months. Correlation of IHTG and plasma Fgf21 for different dietary interventions (B) and ages (C). (D) Plasma Fgf21 was positively correlated with IHTG levels at older ages, while the young 6-month-old mice had elevated plasma Fgf21, despite their low IHTG levels (in red symbols). $r$ values were calculated with Pearson's correlations and their significance are indicated in the parentheses. 

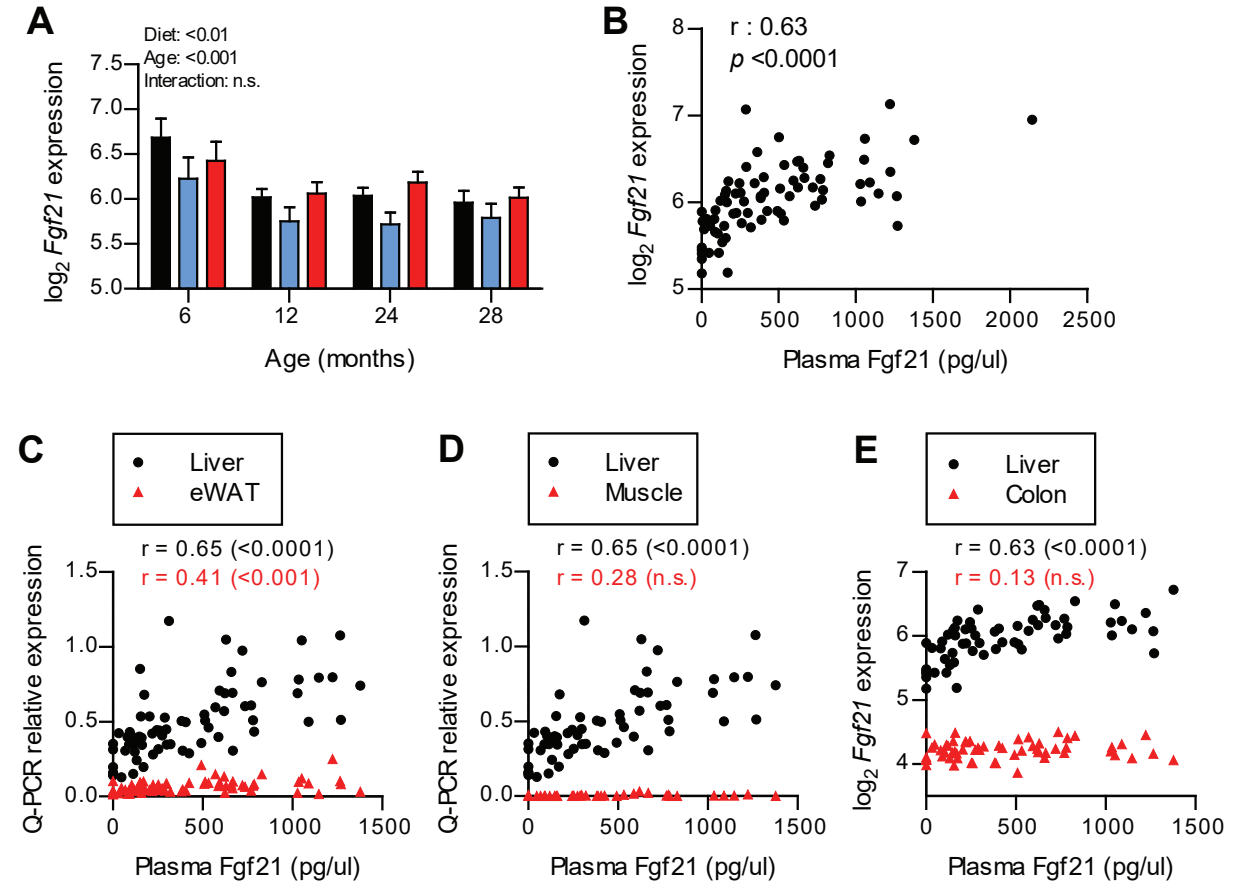

Figure 3. Plasma Fgf21 levels were strongly reflected by the expression of $\boldsymbol{F g f 2 1}$ in the liver. (A) Different expression levels of Fgf21 in the liver induced by the dietary interventions and age. (B) Significant positive correlation between plasma Fgf21 and Fgf21 expression in the liver. The association between plasma and expression levels of Fgf21 was also compared in other tissue types, (C) epidydimal white adipose tissue (eWAT), (D) muscle, and (E) colon tissue. $r$ values were calculated with Pearson's correlations and their significance are indicated in the parentheses.

Subsequently, AUROC analysis was performed using optimal cut-off points determined by the Youden Index (plasma Fgf21 $>222.0$ pg/ml). The results summarized in Table 1 show that the sensitivity was remarkably high at $91.4 \%$, while the specificity was low (57.1\%). This resulted in an AUROC of 0.77 (Supplementary Fig. S3A) and an accuracy of $71.4 \%$ (positive predictive value/ PPV of $60.4 \%$ and negative predictive value/NPV of $90.3 \%$ ). Since plasma Fgf21 appears to perform differently at the age of 6 months and a previous study of obese children indicated that plasma Fgf2 1 did not provide additional value in predicting NAFLD [33], we also tested the Fgf21 performance in older animals only. When the 6 months old animals were excluded, the AUROC and specificity of Fgf21 were improved to 0.84 (Supplementary Fig. S3B) and 73.5\%, while the sensitivity remained high (88.6\%). In addition, the accuracy was improved to $81.2 \%$ (PPV of $77.5 \%$ and NPV of $86.2 \%$ ). Diagnosis using more markers commonly yield a better outcome, so we applied combinations of plasma Fgf21 with other plasma or trait markers for NAFLD, i.e. plasma ALT, plasma CK-18 and body weight. The performance accuracy described in Table 2 revealed that the plasma Fgf21 was best combined with plasma ALT (accuracy of $88.4 \%$ ) or body weight (87.0\%), while the combination with plasma CK-18 (81.2\%) did not improve the performance of Fgf21. 
Table 1. Overall performance of plasma Fgf21 levels for the diagnosis of NAFLD

\begin{tabular}{lccccc} 
AUROC & Sensitivity & Specificity & PPV & NPV & Accuracy \\
\hline $\begin{array}{l}\text { All time points } \\
0.77(0.67-0.87)\end{array}$ & $91.4 \%$ & $57.1 \%$ & $60.4 \%$ & $90.3 \%$ & $71.4 \%$ \\
Without 6 months old & & & & & \\
$0.84(0.74-0.94)$ & $88.6 \%$ & $73.5 \%$ & $77.5 \%$ & $86.2 \%$ & $81.2 \%$
\end{tabular}

Table 2. NAFLD diagnosis performance of plasma Fgf21 in combination with other markers

\begin{tabular}{l|rrrrr}
$\begin{array}{l}\text { Fgf21 in combination } \\
\text { with }\end{array}$ & Sensitivity & Specificity & PPV & NPV & Accuracy \\
\hline Plasma ALT & $80.0 \%$ & $97.1 \%$ & $96.6 \%$ & $82.5 \%$ & $88.4 \%$ \\
Plasma CK-18 & $82.9 \%$ & $79.4 \%$ & $80.6 \%$ & $81.8 \%$ & $81.2 \%$ \\
Body weight & $80.0 \%$ & $94.1 \%$ & $93.3 \%$ & $82.1 \%$ & $87.0 \%$
\end{tabular}




\section{Gene module strongly correlated with plasma Fgf21 level were steatosis- and cancer-related genes}

To explore the molecular mechanism underlying the involvement of Fgf21 in NAFLD development, microarray analysis was performed on mRNA isolated from the livers of the 12, 24, and 28-month old animals. In the search for the molecular processes underlying the association of plasma Fgf21 with NAFLD, the microarray data were analysed by a two-step approach: 1) gene co-expression network analysis using WGCNA [19] and 2) functional pathways and up-stream regulator analysis were determined by applying Ingenuity pathway analysis (IPA) on the co-expression network.

The WGCNA results presented in Figure 4A, show that 5 modules were created from the hepatic transcriptome data and that 3 of them significantly correlated with plasma Fgf21 ( $p<0.05$; module grey denotes background genes outside of modules). The module displaying the most significant correlation with plasma Fgf21 was MEturquoise $(r=-0.61)$. Interestingly, cluster differentiation 36 (Cd36), a fatty acid transporter gene involved in steatosis development, was identified as the top regulated gene in this module. A correlation analysis between plasma Fgf21 and Cd36 expression levels showed a positive significant association (Fig. 4B). Intriguingly, $\beta$-Klotho gene (Klb), a coreceptor component that is required for Fgf21 metabolic activity [34], was included in this module. The expression of Klb decreased with the increase of plasma Fgf21 (Fig. 4C). In addition to plasma Fgf21, strong inverse correlations with Klb expression were also observed for body weight and IHTG (Supplementary Fig. S4A). We also examined plasma Fgf21 correlation with the liver gene expression levels of the 4 members of the Fgf receptor family and observed a significant negative correlation with Fgfr2 and Fgfr4 expression, similarly to Klb (Supplementary Fig. S4B).

Next, we investigated which biological processes are represented by the genes in the MEturquoise module, by using IPA. Figure 4D shows the liver-specific functions with $p$-value $<0.01$ and the 5 most significant regulators identified by IPA, which were ranked by $p$-value. Predicted activation/inhibition z-scores are displayed when available. The liver-specific functions associated with this module included hepatic steatosis and cholestasis, but the most significant function was the hepatocellular carcinoma (HCC). A number of genes related to HCC functions were identified in this module, including collagen type I, alpha 2 (Colla2), matrix metallopeptidase 14 (Mmp14), frizzled-related protein (Frzb), dickkopf WNT signalling pathway inhibitor 3 (Dkk3), glutamateammonia ligase (Glul), and cyclin D1 (Ccnd1). The functions of these genes include extracellular matrix formation/angiogenesis (Colla2 and Mmp14), inhibitors of Wnt signalling (Frzb and Dkk3), and regulation of Wnt target genes (Glul and Ccnd1). The expression levels of these genes were significantly correlated with plasma Fgf21 levels (Fig. 4E), demonstrating that plasma Fgf21 level is associated with HCC-related signalling. Moreover, the identification of predicted upstream 
regulators further confirmed the association of this module with hepatocellular carcinoma (Fig. 4D): predicted inhibition of hepatocyte nuclear factor $4 a$ and 1a (HNF4A and HNF1A), both are tumor suppressor regulators, while rapamycin-insensitive companion of mTOR (RICTOR) and mitogen-activated protein 4 kinase 4 (MAP4K4), factors involved in cancer development, were activated. X-box binding protein (XBP1) was also identified as one of the top upstream regulators.
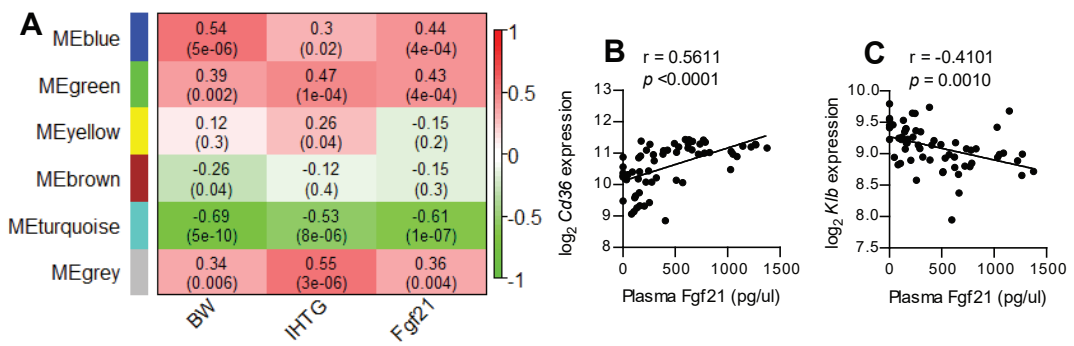

\begin{tabular}{|c|c|c|c|c|}
\hline IPA results & \multicolumn{2}{|c|}{ Functions/upstream regulators } & $p$-value & z score \\
\hline $\begin{array}{l}\text { Liver-specific } \\
\text { functions (\# } \\
\text { of genes) }\end{array}$ & $\begin{array}{l}\text { Hepatocellular ca } \\
\text { Progressive famil } \\
\text { Liver tumor (1164 } \\
\text { Liver cancer (115 } \\
\text { Intrahepatic chole } \\
\text { Hepatic steatosis } \\
\text { Cholestasis (28) }\end{array}$ & $\begin{array}{l}\text { cinoma (1129) } \\
\text { al intrahepatic cholestasis type } 1 \text { (24) } \\
\text { stasis (26) } \\
72 \text { ) }\end{array}$ & $\begin{array}{l}4.96 \times 10^{-6} \\
1.34 \times 10^{-5} \\
2.05 \times 10^{-5} \\
3.17 \times 10^{-5} \\
6.95 \times 10^{-5} \\
4.19 \times 10^{-3} \\
5.29 \times 10^{-3}\end{array}$ & $\begin{array}{c}0.783 \\
\text { NA } \\
1.691 \\
1.391 \\
\text { NA } \\
2.184 \\
0.927\end{array}$ \\
\hline $\begin{array}{l}\text { Upstream } \\
\text { regulators } \\
\text { (\# of genes) }\end{array}$ & $\begin{array}{l}\text { HNF4A (578) } \\
\text { XBP1 (73) } \\
\text { HNF1A (123) } \\
\text { RICTOR (82) } \\
\text { MAP4K4 (44) }\end{array}$ & & $\begin{array}{l}2.49 \times 10^{-51} \\
1.21 \times 10^{-12} \\
1.09 \times 10^{-10} \\
7.07 \times 10^{-10} \\
5.64 \times 10^{-9} \\
\end{array}$ & $\begin{array}{l}-4.300 \\
-6.617 \\
-6.485 \\
6.389 \\
5.729 \\
\end{array}$ \\
\hline 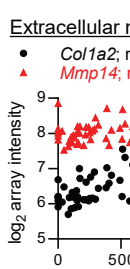 & $\begin{array}{l}\text { atrix/ angiogenesis } \\
=0.50(<0.0001) \\
=0.47(<0.001) \\
\\
1000\end{array}$ & $\begin{array}{l}\text { Wnt inhibitors } \\
\quad \text { Frzb; } r=0.39(<0.01) \\
\text { Dkk3;r=0.34(<0.01) }\end{array}$ & $\begin{array}{l}\text { Wnt target g } \\
\quad \text { Glul; r } \\
\text { Ccnd }\end{array}$ & $\begin{array}{l}8(<0.01) \\
31(<0.05) \\
1000+1500\end{array}$ \\
\hline
\end{tabular}

Figure 4. Liver biological processes associated with plasma Fgf21 level. (A) Heatmap depicting the correlation between gene modules (in rows) and phenotypes (in columns). The top values in each cell represents the correlation coefficient between the module and phenotype with the correlation p-value in parentheses. Red and green color represents positive and negative correlation, respectively. Correlation between plasma Fgf21 levels and hepatic expressions of $\mathrm{Cd} 36$ (B) and $K l b$ (C). (D) Biological processes and regulators associated with MEturquoise, which is the most significant modules correlated with plasma Fgf21. Significant liver-specific functions and upstream regulators are reported in $p$-values and z-scores. Positive and negative z-score represent predicted activation and inhibition, respectively. (E) Correlation between plasma Fgf21 and expression levels of hepatocellular carcinoma-related genes within MEturqouise, which included genes related to extracellular matrix formation and angiogenesis (Colla2 and Mmp14), inhibition of Wnt signalling (Frzb and Dkk3), and downstream target of Wnt signalling (Glul and Ccnd1). r values were calculated with Pearson's correlations and their significance are indicated in the parentheses. 


\section{The elevated plasma Fgf21 levels in young animals without accumulation of IHTG was related to the up-regulation of lipid metabolism by PPARa, PPARGC1a and PPARy}

To elucidate the functions of the genes associated with the elevated Fgf21 plasma level at young age without accumulation of IHTG, WGCNA analysis was performed with the inclusion of the 6-month-old animals. For this purpose, we searched for a gene module that was significantly associated with plasma Fgf21, but not with IHTG, and we found that module MEgreen (1286 genes) fulfilled this criterion (Supplementary Fig. S5A). This module has a significant correlation with Fgf21 ( $r=0.44, p<0.0001)$, but not with IHTG $(r=0.004, p=1)$. To screen for the genes strongly correlated with the plasma Fgf21, we filtered for the genes with a correlation coefficient larger than 0.4. IPA of biological functions revealed that these genes play a role in lipid metabolism (top 3 functions/regulators are listed in Supplementary Fig. S5B) and IPA identified peroxisome proliferator-activated receptor a (PPARa), peroxisome proliferator-activated receptor $\gamma$ coactivator 1-a (PPARGC1a), and PPARy as the predicted upstream regulators.

\section{NRF2 and PPARa targets, pathways differentially up-regulated by NAFLD, were linked to Fgf2 1}

Next, to determine the relevance of Fgf21 in the NAFLD-related pathways, we first identified the pathways differentially regulated in the animals with NAFLD by performing GSEA. Then, we explored the differentially regulated pathways in NAFLD for their link to Fgf21. The gene expression data of 12, 24, and 28-month old animals were analysed with the exclusion of the CR group, since the latter group has a markedly different gene expression profile [20]. Forty-five animals were included in the microarray analysis (32 and 13 animals, with and without NAFLD, respectively). The GSEA results (based on FDR q value $<0.01$ ), presented in Table 3 and $\mathbf{4}$, revealed that 18 and 12 pathways were up- and down-regulated in NAFLD, respectively (lists of pathways with FDR q value $<0.05$ is available in Supplementary Table S4 and S5). The up-regulated pathways were dominated by pathways related to oxidative stress (nuclear factor (erythroid-derived 2)-like 2 or NRF2 targets, glutathione metabolism), energy and lipid metabolism (PPARa targets, oxidative phosphorylation and electron transport chain and fatty acid metabolism). The down-regulated pathways included various complement cascades pathways. Interestingly, NRF2 and PPARa targets, the 2 most significantly enriched up-regulated pathways, contained genes that have been previously identified for their strong correlation with IHTG [35]: NAD(P)H dehydrogenase quinone 1 (Nqo1), sulfiredoxin 1 (Srxn1), cell death-inducing DFFA-like effector a (Cidea) and c (Cidec). Genes in the core enrichment of NRF2 and PPARa targets are listed in Supplementary Table S6 and S7. 
The gene expression levels of these genes were analysed for their correlation with IHTG, which revealed highly significant correlations ( $p<0.0001$; Fig. 5A and B). Although not as strong, the expression levels of these genes were also significantly correlated with the plasma Fgf21 levels. Thus, the differentially regulated PPARa and NRF2 target genes were pointed out to be the link between plasma Fgf21 levels and IHTG content during NAFLD.

Table 3. List of the significantly enriched up-regulated pathways in NAFLD

\begin{tabular}{l|cc}
\multicolumn{1}{l}{ Enriched up-regulated pathways } & NES* & FDR q-value \\
\hline NRF2 TARGETS & 2.617 & 0.00000 \\
PPARA TARGETS & 2.404 & 0.00000 \\
WP1248 OXIDATIVE PHOSPHORYLATION & 2.328 & 0.00000 \\
WP295 ELECTRON TRANSPORT CHAIN & 2.299 & 0.00068 \\
KEGG OXIDATIVE PHOSPHORYLATION & 2.260 & 0.00081 \\
KEGG LYSOSOME & 2.223 & 0.00090 \\
WP1269 FATTY ACID BETA OXIDATION & 2.208 & 0.00097 \\
KEGG FATTY ACID ELONGATION & 2.180 & 0.00135 \\
KEGG FATTY ACID DEGRADATION & 2.146 & 0.00210 \\
MITOCHONDRIAL TRANSLATION & 2.113 & 0.00337 \\
MITOCHONDRIAL TRANSLATION TERMINATION & 2.102 & 0.00344 \\
MAPK TARGETS NUCLEAR EVENTS MEDIATED BY MAP KINASES & 2.079 & 0.00418 \\
KEGG GLUTATHIONE METABOLISM & 2.052 & 0.00625 \\
RESPIRATORY ELECTRON TRANSPORT ATP SYNTHESIS BY & 2.048 & 0.00629 \\
CHEMIOSMOTIC COUPLING AND HEAT PRODUCTION BY & & \\
UNCOUPLING PROTEINS & & \\
SPHINGOLIPID METABOLISM & 2.032 & 0.00775 \\
AQUAPORIN MEDIATED TRANSPORT & 2.025 & 0.00786 \\
KEGG SYNAPTIC VESICLE CYCLE & 2.020 & 0.00795 \\
MITOCHONDRIAL TRANSLATION INITIATION & 2.003 & 0.00937
\end{tabular}

*) Normalised enrichment score (NES); a statistical test for gene set enrichment 
Table 4. List of the significantly enriched down-regulated pathways in NAFLD

\begin{tabular}{l|cc} 
Enriched down-regulated pathways & NES & FDR q-value \\
\hline WP449 COMPLEMENT AND COAGULATION CASCADES & -2.496 & 0.00000 \\
KEGG COMPLEMENT AND COAGULATION CASCADES & -2.496 & 0.00000 \\
KEGG SELENOCOMPOUND METABOLISM & -2.229 & 0.00018 \\
FORMATION OF FIBRIN CLOT CLOTTING CASCADE & -2.272 & 0.00024 \\
WP200 COMPLEMENT ACTIVATION CLASSICAL PATHWAY & -2.219 & 0.00028 \\
BIOC INTRINSICPATHWAY & -2.081 & 0.00213 \\
COMMON PATHWAY & -2.086 & 0.00226 \\
REGULATION OF COMPLEMENT CASCADE & -2.066 & 0.00304 \\
COMPLEMENT CASCADE & -2.023 & 0.00428 \\
AMINO ACID TRANSPORT ACROSS THE PLASMA MEMBRANE & -1.995 & 0.00606 \\
WP460 BLOOD CLOTIING CASCADE & -1.962 & 0.00857 \\
INTRINSIC PATHWAY & -1.963 & 0.00928
\end{tabular}

Notably, MAPK targets was also among the pathways enriched in the NAFLD-differentially regulated pathway (full list of genes in core enrichment of MAPK targets in Supplementary Table S8). This is in line with the finding of MAP4K4 activation in Figure 4D. The top MAPK target genes, protein phosphatase 2 , regulatory subunit $A$, beta $(P p p 2 r 1 b)$ and dual specificity phosphatase 3 (Dusp3), showed significant correlations to both IHTG content and plasma Fgf21 levels (Fig. 5C). Therefore, this signifies the association of plasma Fgf2 1 with liver cancer-related signalling. 


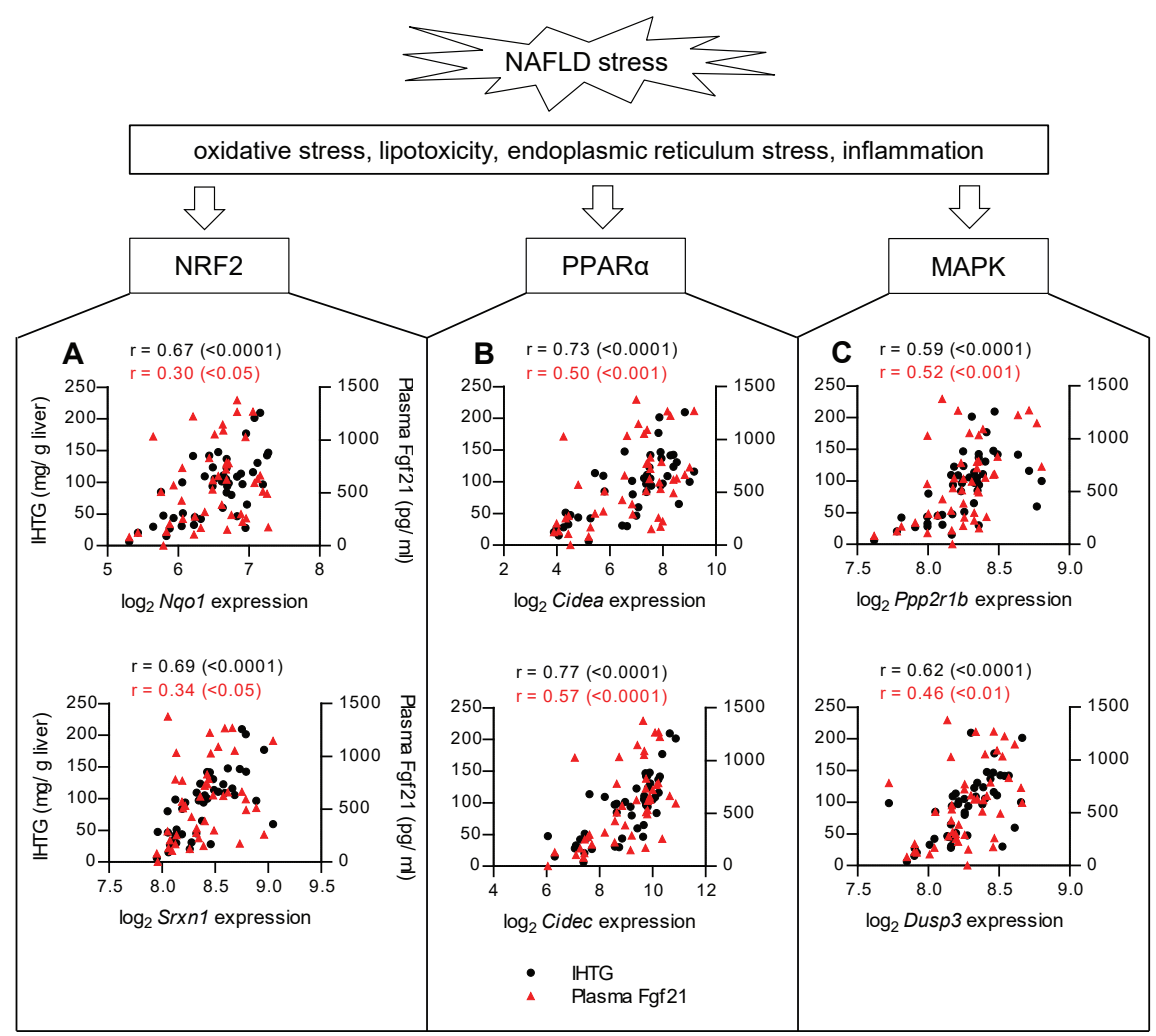

Figure 5. The differentially regulated pathways in NAFLD were reflected by plasma Fgf21 levels. Stress induced by NAFLD may activate NRF2, PPARa and MAPK, and the expression of their target genes. The expression levels of the NRF2 (A), PPARa (B) and MAPK (C) target genes were strongly correlated with IHTG and more modestly with plasma Fgf21 levels (in black and red color, respectively). $r$ values were calculated with Pearson's correlations and their significance are indicated in the parentheses.

\section{Gene expression response to PPARa activation demonstrates its dysregulation in NAFLD}

Based on the essential role of PPARa in lipid homeostasis, we further investigated whether the dysregulation of PPARa in NAFLD extends to an altered response when the system is challenged. To examine the response of PPARa, prior to each sacrifice the PPARa agonist Wy-14,643 (Wy) substance was administered to half of the mice of each intervention group, while the other half of the group received mock treatment. A number of PPARa target genes, including Fgf21 [36, 37], were analysed by Q-PCR, namely monoacylglycerol 0-acyltransferase 1 (Mogat1), G0/G1 switch 2 (G0s2), acyl-CoA thioesterase 3 (Acot3), hydroxymethyl glutary coenzyme A reductase (Hmgcr) (Fig. 6A-E). These genes were selected to represent different functions regulated by PPARa (lipogenesis: Mogat 1; lipolysis: G0s2; fatty acid oxidation: Acot3; cholesterol metabolism: Hmgcr). As shown in Figure 6A, Wy treatment led to induced hepatic Fgf21 expression and the 2-way ANOVA test indicated an 
interaction between NAFLD and the Wy response. Intriguingly, animals with NAFLD exhibited an augmented response to the treatment. Similarly to Fgf21 expression, the induction of Mogat1 and Hmgcr expression levels were stronger in the animals with NAFLD (Fig. 6B and E). However, not all genes demonstrated the stochastic response to Wy treatment. G0s2 and Acot3 expression levels were similarly up-regulated regardless of the presence of NAFLD (Fig. $\mathbf{6 C}$ and D). The expression of Ppara itself was also examined and the results in Figure 6F show that the stimulation of PPARa did not differ between the animals with and without NAFLD. Although NAFLD presence did not alter the response to Wy treatment of all PPARa target genes, the expression profiles of Fgf21, Mogat1 and Hmgcr underscore the dysregulation PPARa signalling pathway in NAFLD.
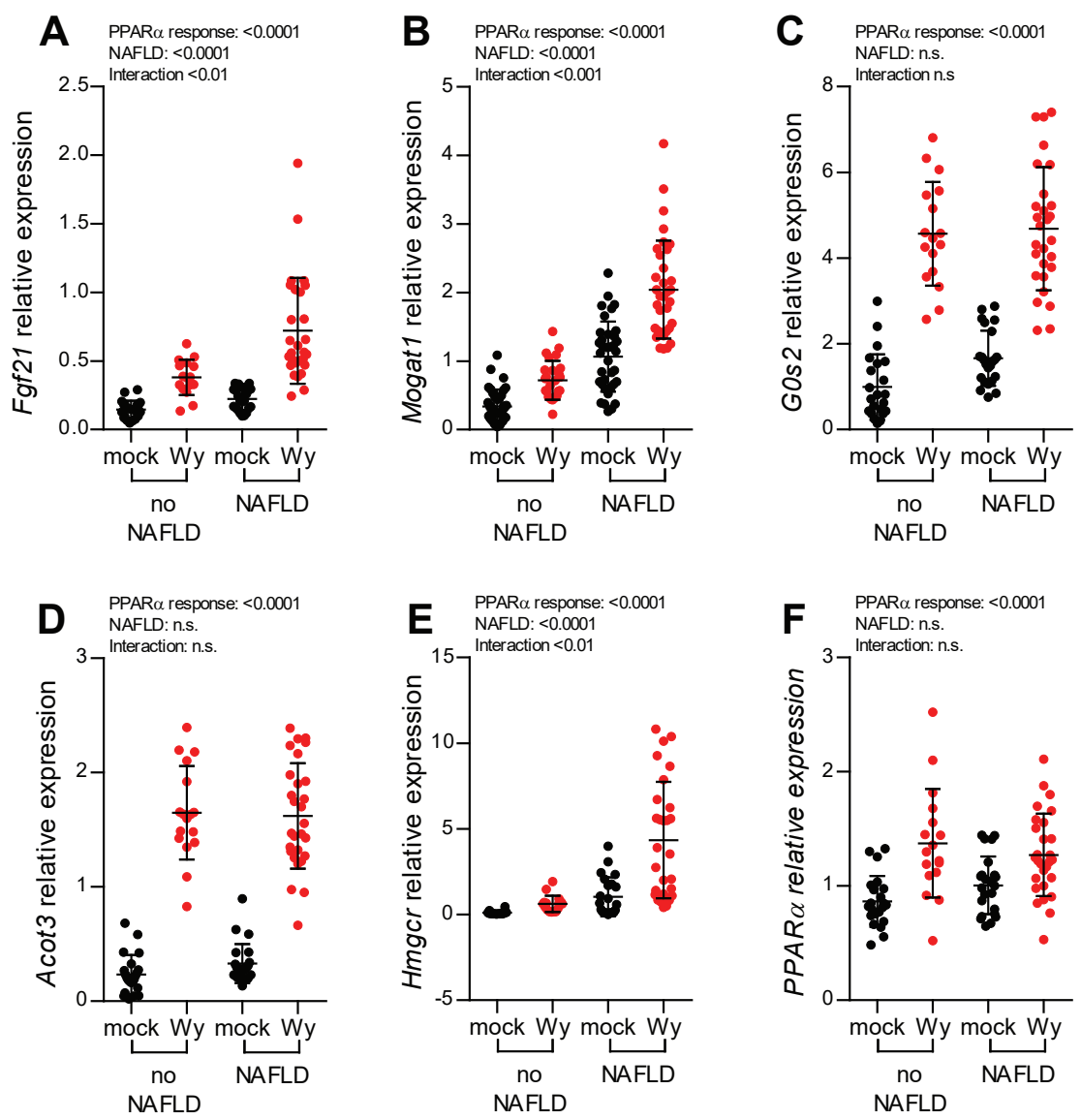

Figure 6. The response to PPARa challenge test showed that the presence of NAFLD partially altered the response at the gene expression levels. (A) Fgf21; (B) Mogat1; (C) GOs2; (D) Acot3; (E) Hmgcr; and (F) Ppara. The effect of PPARa agonist and NAFLD, as well as any interaction between them, were analysed using two-way ANOVA. 


\section{DISCUSSION}

In our mouse aging cohort, NAFLD development started at middle-age in Control (C)- and medium-fat (MF)-exposed mice, but not in the calorie restricted (CR)-fed animals, which stayed lean over time. The prevalence of NAFLD in the ad libitum C- and MF-fed groups increased during aging, which reflects weight gain and aging as risk factors for developing NAFLD[38]. In this study, we applied a long-term exposure to a less extreme diet compared to previous studies $[17,18]$ by using a 25E\% medium-fat diet to mimic the slow onset of NAFLD in the human population, which did not induce severe NAFLD. We assessed this by analysing the liver fibrosis marker, 4-hydroxyproline content, and observed a lower prevalence of liver fibrosis, compared to liver steatosis or benign NAFLD.

The analysis of plasma Fgf21 levels in the different intervention groups and ages suggests that there is an age-effect on plasma Fgf21 levels, although we did not observe a diet-dependent effect on plasma Fgf21 levels. We found that at the age of 6 months, some of the mice displayed a high level of plasma Fgf21 in the absence of elevated IHTG levels. A previous study in children has also revealed a lack of correlation between serum FGF21 and NAFLD parameters [39], moreover another study showed an inverse correlation between FGF21 and hepatic damage [40]. The addition of serum FGF21 in a NAFLD diagnostic model for children and adolescent also failed to improve the diagnostic performance [33]. An age of 6 months in mice is equivalent to mature adult age ( 30 years old in human) [41] and does not correspond to a developmental period in childhood. However, both mice and human data support either an absence of correlation or different correlation between plasma Fgf21 and NAFLD in younger age groups. Overall, our results reveal that, with the exception of the 6-month-old animals, plasma Fgf21 levels significantly correlated with IHTG content and performed well as a plasma marker for NAFLD diagnosis. This is in agreement with the results of previous studies in humans $[10,11]$.

The pathogenesis of NAFLD is attributed to a multi-hit process that includes lipotoxicity, oxidative stress and endoplasmic reticulum (ER) stress. Liver fat accumulation involves excess fatty acid supply to the liver, which triggers fatty acid oxidation and, consequently, oxidative stress from microsomal enzymes and ER stress. We found that NRF2 and PPARa targets were the most significantly enriched up-regulated pathways in the animals with NAFLD. NRF2 acts as a protective measure against oxidative stress, by producing antioxidant proteins. Meanwhile, PPARa activation is crucial for maintaining the homeostasis of fatty acid metabolism by increasing mitochondrial $\beta$-oxidation, thereby reducing the potential for fatty acid-induced lipotoxicity [42]. In addition, the WGCNA followed by pathway analysis pointed XBP1 out as one of the predicted upstream regulators 
of Fgf21. The elevated expression of FGF21 has been described as a counteractive mechanism for ER stress, by modulating lipid metabolism [43-45]. Therefore, the up-regulation of Fgf21 in NAFLD appears to be a simultaneous protection against lipotoxicity, oxidative stress and ER stress in NAFLD.

Intriguingly, we found that plasma Fgf21 levels at young age were related to fatty acid oxidation ( $L$-carnitine shuttle and $\beta$-oxidation), which also corresponds to PPARa activation. While this seems to be contradictory with the idea of PPARa activation as a protective measure for fatty liver, both mouse and human studies have reported that the increased mitochondrial activity and $\beta$-oxidation do not necessarily reflect an efficient electron transport chain [46-48]. The authors found that the electron transport chain in subjects with hepatic steatosis and/or obesity is inefficient [46-48]. Therefore, it is worthwhile noting that, despite the up-regulated fatty acid oxidation in both young animals without NAFLD and old animals with NAFLD, the up-regulation in old animals with NAFLD measurement might lead to perturbing consequences, such as hepatic oxidative stress.

The in vivo PPARa challenge performed in this study provides a novel insight into the ability of maintaining metabolic homeostasis. A dynamic measurement by using system perturbation or challenge tests are likely more valuable to define metabolic health or resilience, compared to more static measurements [49]. By performing the PPARa agonist treatment, we demonstrate that the PPARa response at the transcriptional level is partially altered in the presence of NAFLD. It appears that liver fat is the burden of the liver's plasticity of lipid metabolism. A similar notion was reported by Hyotylainen and co-workers, showing that high liver fat markedly hampers the ability of the liver to adaptively regulate metabolism to meet the excessive demands on basic liver functions. As a consequence, individuals with NAFLD may be more vulnerable to various metabolic stressors on the liver [50]. This underlines that, although hepatic steatosis is considered benign (first hit), when the metabolic system faces a challenge (second/multiple hits), the ability to maintain or regain homeostasis might have been compromised.

Furthermore, we revealed the association between plasma Fgf21 and transcriptional changes related to hepatocellular carcinoma $(\mathrm{HCC})$ development. The predicted regulators (HNF1A, HNF4A, RICTOR, MAP4K4), functional pathways and genes (Wnt target genes Ccnd1 and Glul) associated with plasma Fgf21 suggest dysregulations of metabolic and proliferative pathways [51, 52], which are characteristics of a benign hepatocellular tumor. Although these dysregulations alone were not sufficient to induce carcinogenesis, it likely increases the susceptibility to HCC development. In line with this hypothesis, a pre-malignant stage in NAFLD has been shown to denote stress, inflammation and even apoptosis, which pre-condition and initiate pro-oncogenic signals [53]. This is supported by the evidence that both liver expression and circulating levels of FGF21 are increased 
in patients with hepatitis, cirrhosis and hepatocarcinoma [54]. Therefore, the population would likely benefit from the use of plasma Fgf21 as a biomarker of an early stage of NAFLD.

Although the association between plasma Fgf21 and HCC-related function is not as strong as its performance in reflecting the benign NAFLD stage, plasma Fgf21 might be beneficial in improving the performance of biomarkers for advanced stages of NAFLD. This has been suggested in the study of a NASH biomarker, that for the purpose of defining the stages of NASH, plasma CK-18 performs better than FGF21, but adding FGF21 to the CK-18 model significantly improved the performance [14]. We evaluated the combined analysis of plasma Fgf21 and CK-18 to detect NAFLD in our study, but it did not result in a higher accuracy compared to merely Fgf21 analysis. A plausible explanation for this observation is that our mice cohort modelled a rather mild NAFLD development, so that the addition of plasma CK-18, which represents advanced stages of NAFLD, did not effectively improve the analysis.

In this study, we discovered that the expression level of Klb, a critical co-receptor of the FGF receptors, was negatively correlated with plasma Fgf21, IHTG and body weight. In addition, the liver expression levels of the FGF receptors Fgfr2 and Fgfr4 showed similar patterns, although the declining expression levels were not as strong as observed for Klb. FGFR2 and FGFR4 proteins, along with FGFR1, have been shown to form a transmembrane complex with $\beta$-klotho to mediate the effects of FGF21 in adipocytes [55]. Although the type of FGF receptor that forms a complex with $\beta$-klotho protein in the liver is still unclear, this observation underlines the growing notion that metabolic system might develop a resistance to mediate the beneficial effect of Fgf21. The Fgf21 resistance due to its co-receptor alteration is an essential issue to be addressed during the further development of FGF21 as a novel pharmacological agent for metabolic diseases [56]. Both human and mice studies have reported increased FGF21 gene expression or circulating protein levels with obesity and/or metabolic syndrome [57-59]. One of these studies also demonstrated that the diet-induced obese mice with an elevated endogenous level of Fgf2 1 responded poorly to acute exogenous Fgf21 administration [59]. Since Klb plays a critical role in mediating Fgf21's metabolic activity $[34,60]$, the declining Klb expression that occurs over a long-term obesity and/ or hepatic steatosis development might result in Fgf21 resistance. Therefore, in order to assess the possibility of resistance to FGF21 treatment, the consequence of the down-regulation of critical FGF21 receptors/co-receptors in the liver on sensitivity to endo- and exogenous FGF21 warrants further investigation. It is worthwhile noting that, in the acute induction of obesity in mouse model, Klb expression was not altered in the obese state [59].

Taken together, in this study, we demonstrate that plasma Fgf21 levels strongly reflects liver fat accumulation, confirming its potential as NAFLD marker. However, this association is age- 
dependent and does not apply at the age of 6 months in the C57BL/6J mice. The molecular link between plasma Fgf21 and IHTG levels was associated with dysregulation of both metabolic and cancer-related pathways. The up-regulated Fgf21 levels in NAFLD appears to be a measure to maintain homeostasis against the adverse effects in NAFLD, e.g. lipotoxicity, oxidative stress and endoplasmic reticulum stress. The elevated plasma Fgf2 1 is also associated with declining expression of $K l b$, its crucial co-receptor, which suggests a resistance to Fgf21. Therefore, although liver fat accumulation is a benign stage of NAFLD, the liver is likely more vulnerable to metabolic stressors and progress to end-stage liver disease. The in vivo PPARa challenge further demonstrates the dysregulation of PPARa signalling in the presence of NAFLD, which results in a stochastically increasing hepatic expression of Fgf21. In conclusion, Fgf21 plasma levels reflect liver fat accumulation and dysregulation of metabolic pathways at a transcriptional level in the liver of C57BL/6J mice. 


\section{REFERENCES}

[1] Lazo, M., Hernaez, R., Eberhardt, M. S., Bonekamp, S., et al., Prevalence of Nonalcoholic Fatty Liver Disease in the United States: The Third National Health and Nutrition Examination Survey, 1988-1994. American Journal of Epidemiology 2013, 178, 38-45.

[2] Satapathy, S. K., Sanyal, A. J., Epidemiology and Natural History of Nonalcoholic Fatty Liver Disease. Semin Liver Dis 2015, 35, 221-235.

[3] Marchesini, G., Petta, S., dale Grave, R., Diet, Weight Loss, and Liver Health in NAFLD: Pathophysiology, Evidence and Practice. Hepatology 2015, n/a-n/a.

[4] Yan, H., Xia, M., Chang, X., Xu, Q., et al., Circulating fibroblast growth factor 21 levels are closely associated with hepatic fat content: a cross-sectional study. PloS one 2011, 6, e24895.

[5] Yano, E., Tagawa, K., Yamaoka, K., Mori, M., Test validity of periodic liver function tests in a population of Japanese male bank employees. J. Clin. Epidemiol. 2001, 54, 945-951.

[6] Mofrad, P., Contos, M. J., Haque, M., Sargeant, C., et al., Clinical and histologic spectrum of nonalcoholic fatty liver disease associated with normal ALT values. Hepatology 2003, 37, 1286-1292.

[7] Angulo, P., Nonalcoholic fatty liver disease. New England Journal of Medicine 2002, 346, 1221-1231.

[8] Chalasani, N., The diagnosis and management of non-alcoholic fatty liver disease: practice guideline by the American Gastroenterological Association, American Association for the Study of Liver Diseases, and American College of Gastroenterology. 2012, 142, 1592-1609.

[9] Saadeh, S., Younossi, Z. M., Remer, E. M., Gramlich, T., et al., The utility of radiological imaging in nonalcoholic fatty liver disease. Gastroenterology 2002, 123, 745-750.

[10] Dushay, J., Chui, P. C., Gopalakrishnan, G. S., Varela-Rey, M., et al., Increased fibroblast growth factor 21 in obesity and nonalcoholic fatty liver disease. Gastroenterology 2010, 139, 456-463.

[11] Li, H., Fang, Q., Gao, F., Fan, J., et al., Fibroblast growth factor 21 levels are increased in nonalcoholic fatty liver disease patients and are correlated with hepatic triglyceride. Journal of Hepatology 2010, 53, 934-940.

[12] Yilmaz, Y., Eren, F., Yonal, O., Kurt, R., et al., Increased serum FGF21 levels in patients with nonalcoholic fatty liver disease. European journal of clinical investigation 2010, 40, 887-892.

[13] Li, H., Dong, K., Fang, Q., Hou, X., et al., High serum level of fibroblast growth factor 21 is an independent predictor of non-alcoholic fatty liver disease: A 3-year prospective study in China. J. Hepatol. 2013, 58, 557-563.

[14] Shen, J., Chan, H. L.-Y., Wong, G. L.-H., Choi, P. C.-L., et al., Non-invasive diagnosis of non-alcoholic steatohepatitis by combined serum biomarkers. Journal of Hepatology 2012, 56, 1363-1370.

[15] Li, H., Zhang, J., Jia, W., Fibroblast growth factor 21: a novel metabolic regulator from pharmacology to physiology. Frontiers of Medicine 2013, 7, 25-30.

[16] Bellentani, S., Scaglioni, F., Marino, M., Bedogni, G., Epidemiology of Non-Alcoholic Fatty Liver Disease. Dig Dis 2010, 28, 155-161.

[17] Duval, C., Thissen, U., Keshtkar, S., Accart, B., et al., Adipose Tissue Dysfunction Signals Progression of Hepatic Steatosis Towards Nonalcoholic Steatohepatitis in C57Bl/6 Mice. Diabetes 2010, 59, 3181-3191.

[18] Buettner, R., Schölmerich, J., Bollheimer, L. C., High-fat Diets: Modeling the Metabolic Disorders of Human Obesity in Rodents. Obesity 2007, 15, 798-808.

[19] Langfelder, P., Horvath, S., WGCNA: an R package for weighted correlation network analysis. BMC Bioinformatics 2008, 9, 559.

[20] Rusli, F., Boekschoten, M. V., Zubia, A. A., Lute, C., et al., A weekly alternating diet between caloric restriction and medium fat protects the liver from fatty liver development in middle-aged C57BL/6J mice. Molecular nutrition \& food research 2015, 59, 533-543. 
[21] van Norren, K., Rusli, F., van Dijk, M., Lute, C., et al., Behavioural changes are a major contributing factor in the reduction of sarcopenia in caloric-restricted ageing mice. Journal of Cachexia, Sarcopenia and Muscle 2015, n/a-n/a.

[22] Kleiner, D. E., Brunt, E. M., Van Natta, M., Behling, C., et al., Design and validation of a histological scoring system for nonalcoholic fatty liver disease. Hepatology 2005, 41, 1313-1321.

[23] Meyer, T. E., Kovács, S. J., Ehsani, A. A., Klein, S., et al., Long-Term Caloric Restriction Ameliorates the Decline in Diastolic Function in Humans. Journal of the American College of Cardiology 2006, 47, 398-402.

[24] Steegenga, W. T., Mischke, M., Lute, C., Boekschoten, M. V., et al., Sexually dimorphic characteristics of the small intestine and colon of prepubescent C57BL/6 mice. Biology of Sex Differences 2014, 5, 1-17.

[25] Lin, K., Kools, H., de Groot, P. J., Gavai, A. K., et al., MADMAX - Management and analysis database for multiple omics experiments. Journal of integrative bioinformatics 2011, 8, 160.

[26] Bolstad, B. M., Irizarry, R. A., Åstrand, M., Speed, T. P., A comparison of normalization methods for high density oligonucleotide array data based on variance and bias. Bioinformatics 2003, 19, 185-193.

[27] Irizarry, R. A., Bolstad, B. M., Collin, F., Cope, L. M., et al., Summaries of Affymetrix GeneChip probe level data. Nucleic Acids Research 2003, 31, e15.

[28] Dai, M., Wang, P., Boyd, A. D., Kostov, G., et al., Evolving gene/transcript definitions significantly alter the interpretation of GeneChip data. Nucleic Acids Research 2005, 33, e175.

[29] Wang, X., Seed, B., A PCR primer bank for quantitative gene expression analysis. Nucleic Acids Research 2003, 31, e154.

[30] Ritchie, M. E., Phipson, B., Wu, D., Hu, Y., et al., limma powers differential expression analyses for RNAsequencing and microarray studies. Nucleic acids research 2015, 43, e47.

[31] Sartor, M. A., Tomlinson, C. R., Wesselkamper, S. C., Sivaganesan, S., et al., Intensity-based hierarchical Bayes method improves testing for differentially expressed genes in microarray experiments. BMC Bioinformatics 2006, 7.

[32] Mattson, M. P., Challenging Oneself Intermittently to Improve Health. Dose-Response 2014, 12.

[33] Koot, B. G., van der Baan-Slootweg, O. H., Bohte, A. E., Nederveen, A. J., et al., Accuracy of prediction scores and novel biomarkers for predicting nonalcoholic fatty liver disease in obese children. Obesity 2013, $21,583-590$.

[34] Ogawa, Y., Kurosu, H., Yamamoto, M., Nandi, A., et al., BKlotho is required for metabolic activity of fibroblast growth factor 21. Proceedings of the National Academy of Sciences 2007, 104, 7432-7437.

[35] Hui, S. T., Parks, B. W., Org, E., Norheim, F., et al., The genetic architecture of NAFLD among inbred strains of mice. eLife 2015, 4.

[36] Badman, M. K., Pissios, P., Kennedy, A. R., Koukos, G., et al., Hepatic fibroblast growth factor 21 is regulated by PPARa and is a key mediator of hepatic lipid metabolism in ketotic states. Cell metabolism 2007, 5, 426-437.

[37] Inagaki, T., Dutchak, P., Zhao, G., Ding, X., et al., Endocrine regulation of the fasting response by PPARamediated induction of fibroblast growth factor 21. Cell metabolism 2007, 5, 415-425.

[38] Duseja, A., Chalasani, N., Epidemiology and risk factors of nonalcoholic fatty liver disease (NAFLD). Hepatology International 2013, 7, 755-764.

[39] Reinehr, T., Woelfle, J., Wunsch, R., Roth, C. L., Fibroblast growth factor 21 (FGF-21) and its relation to obesity, metabolic syndrome, and nonalcoholic fatty liver in children: a longitudinal analysis. The Journal of Clinical Endocrinology \& Metabolism 2012, 97, 2143-2150. 
[40] Alisi, A., Ceccarelli, S., Panera, N., Prono, F., et al., Association between serum atypical fibroblast growth factors 21 and 19 and pediatric nonalcoholic fatty liver disease. PloS one 2013, 8, e67160.

[41] Flurkey, K., M. Currer, J., Harrison, D. E., James, G. F., et al., The Mouse in Biomedical Research (Second Edition), Academic Press, Burlington 2007, pp. 637-672.

[42] Berson, A., De Beco, V., Lettéron, P., Robin, M. A., et al., Steatohepatitis-inducing drugs cause mitochondrial dysfunction and lipid peroxidation in rat hepatocytes. Gastroenterology 1998, 114, 764-774.

[43] Schaap, F. G., Kremer, A. E., Lamers, W. H., Jansen, P. L., Gaemers, I. C., Fibroblast growth factor 21 is induced by endoplasmic reticulum stress. Biochimie 2013, 95, 692-699.

[44] Kim, S., Kim, K., Kim, H.-K., Kim, M.-J., et al., Fibroblast growth factor 21 participates in adaptation to endoplasmic reticulum stress and attenuates obesity-induced hepatic metabolic stress. Diabetologia 2015, 58, 809-818.

[45] Jiang, S., Yan, C., Fang, Q.-C., Shao, M.-l., et al., Fibroblast Growth Factor 21 Is Regulated by the IRE1 a-XBP1 Branch of the Unfolded Protein Response and Counteracts Endoplasmic Reticulum Stress-induced Hepatic Steatosis. J. Biol. Chem. 2014, 289, 29751-29765.

[46] Sunny, Nishanth E., Parks, Elizabeth J., Browning, Jeffrey D., Burgess, Shawn C., Excessive Hepatic Mitochondrial TCA Cycle and Gluconeogenesis in Humans with Nonalcoholic Fatty Liver Disease. Cell Metabolism 2011, 14, 804-810.

[47] Benoit, B., Plaisancié, P., Awada, M., Géloën, A., et al., High-fat diet action on adiposity, inflammation, and insulin sensitivity depends on the control low-fat diet. Nutrition Research 2013, 33, 952-960.

[48] Forster, M. J., Sohal, B. H., Sohal, R. S., Reversible Effects of Long-Term Caloric Restriction on Protein Oxidative Damage. The Journals of Gerontology Series A: Biological Sciences and Medical Sciences 2000, 55, B522-B529.

[49] van Ommen, B., van der Greef, J., Ordovas, J. M., Daniel, H., Phenotypic flexibility as key factor in the human nutrition and health relationship. Genes \& Nutrition 2014, 9, 1-9.

[50] Hyotylainen, T., Jerby, L., Petaja, E. M., Mattila, I., et al., Genome-scale study reveals reduced metabolic adaptability in patients with non-alcoholic fatty liver disease. Nat Commun 2016, 7.

[51] Odom, D. T., Zizlsperger, N., Gordon, D. B., Bell, G. W., et al., Control of Pancreas and Liver Gene Expression by HNF Transcription Factors. Science 2004, 303, 1378-1381.

[52] Nault, J. C., Bioulac-Sage, P., Zucman-Rossi, J., Hepatocellular Benign Tumors-From Molecular Classification to Personalized Clinical Care. Gastroenterology 2013, 144, 888-902.

[53] Stickel, F., Hellerbrand, C., Non-alcoholic fatty liver disease as a risk factor for hepatocellular carcinoma: mechanisms and implications. Gut 2010.

[54] Yang, C., Lu, W., Lin, T., You, P., et al., Activation of Liver FGF21 in hepatocarcinogenesis and during hepatic stress. BMC gastroenterology 2013, 13, 67.

[55] Kharitonenkov, A., Dunbar, J. D., Bina, H. A., Bright, S., et al., FGF-21/FGF-21 receptor interaction and activation is determined by $\beta$ Klotho. Journal of cellular physiology 2008, 215, 1-7.

[56] Kharitonenkov, A., DiMarchi, R., FGF21 Revolutions: Recent Advances Illuminating FGF21 Biology and Medicinal Properties. Trends Endocrinol Metab 2015, 26, 608-617.

[57] Muise, E. S., Azzolina, B., Kuo, D. W., El-Sherbeini, M., et al., Adipose Fibroblast Growth Factor 21 Is UpRegulated by Peroxisome Proliferator-Activated Receptor $\mathrm{Y}$ and Altered Metabolic States. Molecular Pharmacology 2008, 74, 403-412.

[58] Zhang, X., Yeung, D. C. Y., Karpisek, M., Stejskal, D., et al., Serum FGF21 Levels Are Increased in Obesity and Are Independently Associated With the Metabolic Syndrome in Humans. Diabetes 2008, 57, 1246-1253.

[59] Fisher, f. M., Chui, P. C., Antonellis, P. J., Bina, H. A., et al., Obesity Is a Fibroblast Growth Factor 21 (FGF21)Resistant State. Diabetes 2010, 59, 2781-2789.

[60] Ding, X., Boney-Montoya, J., Owen, Bryn M., Bookout, Angie L., et al., $\beta K$ lotho Is Required for Fibroblast Growth Factor 21 Effects on Growth and Metabolism. Cell Metabolism 2012, 16, 387-393. 


\section{ACKNOWLEDGEMENTS}

Financial support: This work was financially supported by European Union's Seventh Framework Programme (FP7/2007-2011) IDEAL-aging under grant agreement no. 259679.

\section{AUTHOR CONTRIBUTIONS}

F.R. and J.D. conceived the study and designed the experiment with input from W.T.S. and M.B.. F.R., E.A. and C.L. performed the experiments and data analysis. M.V.B and E.B.vd.A. supervised the data analysis. F.R., J.D. and E.A. wrote the manuscript with contributions from all authors. M.M., M.B. and W.T.S. supervised the entire project.

\section{ADDITIONAL INFORMATION}

Competing financial interests: The authors declare no competing financial interests. 


\section{SUPPLEMENTARY FIGURES}

Supplementary Table S1. Composition of the experimental diet. The CR diet was adjusted for the vitamins and minerals amount to ensure a homologous intake between both groups.

\begin{tabular}{l|ccc}
\multicolumn{2}{c}{ AIN-93W } & AIN-93W-CR & AIN-93W-MF \\
\hline Energy (kcal/g) & 3.85 & 3.77 & 4.25 \\
Energy from fat (\%) & 9 & 10 & 25 \\
Energy from protein (\%) & 15 & 15 & 13 \\
Energy from carbohydrates (\%) & 76 & 75 & 61 \\
Mineral mix AIN-93M (g\%) & 35 & 50 & 35 \\
Vitamin mix AIN-93M (g\%) & 10 & 14 & 10 \\
Choline bitartrate (g\%) & 2.5 & 3.5 & 2.5
\end{tabular}

Supplementary Table S2. List of primer sequence used in Q-PCR analysis

\begin{tabular}{l|ll}
\multicolumn{1}{l}{ Gene name } & \multicolumn{1}{c}{ Forward primer $\left(\mathbf{5}^{\prime} \boldsymbol{\rightarrow} \mathbf{3}^{\prime} \mathbf{)}\right.$} & \multicolumn{1}{c}{ Reverse primer $\left(\mathbf{5}^{\prime} \boldsymbol{\rightarrow} \mathbf{3}^{\prime}\right)$} \\
\hline Fgf21 & GTG-TCA-AAG-CCT-CTA-GGT-TTC-TT & GGT-ACA-CAT-TGT-AAC-CGT-CCT- C \\
Mogat1 & TCC-CGT-TGT-TCC-GAG-AAT-ATC-T & TGC-TCA-GCA-CAT-GAG-ACA-AAC \\
G0s2 & AGT-GCT-GCC-TCT-CTT-CCC-AC & TT-CCA-TCT-GAG-CTC-TGG-GC \\
Acot3 & TCC-AAC-ATC-GGC-GGA-AAC-TTA & ACG-GGA-ATC-AAG-CTC-TTC-TGG \\
Hmgcr & AGC-TTG-CCC-GAA-TTG-TAT-GTG & TCT-GTT-GTG-AAC-CAT-GTG-ACT-TC \\
Ppar $\alpha$ & TAT-TCG-GCT-GAA-GCT-GGT-GTA-C & CTG-GCA-TTT-GTT-CCG-GT-CT \\
Rplp0 & ATG-GGT-ACA-AGC-GCG-TCC-TG & GCC-TTG-ACC-TTT-TCA-GTA-AG
\end{tabular}

Supplementary Table S3. Characteristics of animals with/without NAFLD

\begin{tabular}{l|ccc}
\multicolumn{1}{c}{} & $\begin{array}{c}\text { Without NAFLD } \\
(\mathrm{n}=53)\end{array}$ & $\begin{array}{c}\text { NAFLD } \\
(\mathrm{n}=36)\end{array}$ & p-value \\
\hline Body weight $(\mathrm{g})$ & $28.9 \pm 0.9$ & $44.5 \pm 1.2$ & $<0.0001$ \\
eWAT weight (g) & $0.46 \pm 0.05$ & $1.44 \pm 0.59$ & $<0.0001$ \\
Relative eWAT weight (\%) & $1.43 \pm 0.11$ & $3.19 \pm 0.17$ & $<0.0001$ \\
Liver weight (g) & $1.02 \pm 0.04$ & $1.71 \pm 0.07$ & $<0.0001$ \\
Relative liver weight (\%) & $3.50 \pm 0.09$ & $3.86 \pm 0.15$ & 0.0341 \\
Fasting plasma insulin (ng/ml) & $0.627 \pm 0.093$ & $1.767 \pm 0.283$ & $<0.0001$ \\
Plasma ALT (U/l) & $6.82 \pm 0.72$ & $11.17 \pm 1.03$ & 0.0006 \\
IHTG (mg/g liver) & $22.2 \pm 1.7$ & $111.1 \pm 6.2$ & $<0.0001$ \\
Liver hydroxyproline ( $\mu \mathrm{g} / \mathrm{mg} \mathrm{liver)}$ & $0.140 \pm 0.008$ & $0.192 \pm 0.015$ & 0.0013
\end{tabular}

Data are mean \pm s.e.m.; $p$-values are t-test between animals with and without NAFLD 
Supplementary Table S4. List of the significantly enriched up-regulated pathways in NAFLD (FDR q-value <0.05)

\begin{tabular}{|c|c|c|}
\hline Enriched up-regulated pathways & NES & $\begin{array}{l}\text { FDR q- } \\
\text { value }\end{array}$ \\
\hline NRF2 TARGETS & 2.617 & 0.00000 \\
\hline PPARA TARGETS & 2.404 & 0.00000 \\
\hline WP1248 OXIDATIVE PHOSPHORYLATION & 2.328 & 0.00000 \\
\hline WP295 ELECTRON TRANSPORT CHAIN & 2.299 & 0.00068 \\
\hline KEGG OXIDATIVE PHOSPHORYLATION & 2.260 & 0.00081 \\
\hline KEGG LYSOSOME & 2.223 & 0.00090 \\
\hline WP1269 FATTY ACID BETA OXIDATION & 2.208 & 0.00097 \\
\hline KEGG FATTY ACID ELONGATION & 2.180 & 0.00135 \\
\hline KEGG FATTY ACID DEGRADATION & 2.146 & 0.00210 \\
\hline MITOCHONDRIAL TRANSLATION & 2.113 & 0.00337 \\
\hline MITOCHONDRIAL TRANSLATION TERMINATION & 2.102 & 0.00344 \\
\hline MAPK TARGETS NUCLEAR EVENTS MEDIATED BY MAP KINASES & 2.079 & 0.00418 \\
\hline KEGG GLUTATHIONE METABOLISM & 2.052 & 0.00625 \\
\hline $\begin{array}{l}\text { RESPIRATORY ELECTRON TRANSPORT ATP SYNTHESIS BY CHEMIOSMOTIC } \\
\text { COUPLING AND HEAT PRODUCTION BY UNCOUPLING PROTEINS }\end{array}$ & 2.048 & 0.00629 \\
\hline SPHINGOLIPID METABOLISM & 2.032 & 0.00775 \\
\hline AQUAPORIN MEDIATED TRANSPORT & 2.025 & 0.00786 \\
\hline KEGG SYNAPTIC VESICLE CYCLE & 2.020 & 0.00795 \\
\hline MITOCHONDRIAL TRANSLATION INITIATION & 2.003 & 0.00937 \\
\hline MEMBRANE TRAFFICKING & 1.991 & 0.01022 \\
\hline MITOCHONDRIAL TRANSLATION ELONGATION & 1.984 & 0.01085 \\
\hline BIOC MPRPATHWAY & 1.975 & 0.01157 \\
\hline REGULATION OF ACTIN DYNAMICS FOR PHAGOCYTIC CUP FORMATION & 1.962 & 0.01232 \\
\hline IRON UPTAKE AND TRANSPORT & 1.963 & 0.01280 \\
\hline MHC CLASS II ANTIGEN PRESENTATION & 1.964 & 0.01319 \\
\hline TRANSFERRIN ENDOCYTOSIS AND RECYCLING & 1.943 & 0.01459 \\
\hline RESPIRATORY ELECTRON TRANSPORT & 1.945 & 0.01479 \\
\hline APOPTOTIC EXECUTION PHASE & 1.928 & 0.01634 \\
\hline KEGG PPAR SIGNALING PATHWAY & 1.884 & 0.01960 \\
\hline PHAGOSOMAL MATURATION EARLY ENDOSOMAL STAGE & 1.910 & 0.01961 \\
\hline WP2316 PPAR SIGNALING PATHWAY & 1.887 & 0.01962 \\
\hline KEGG SPHINGOLIPID METABOLISM & 1.887 & 0.02005 \\
\hline PROSTACYCLIN SIGNALLING THROUGH PROSTACYCLIN RECEPTOR & 1.889 & 0.02012 \\
\hline CAM PATHWAY & 1.890 & 0.02023 \\
\hline KEGG VASOPRESSIN REGULATED WATER REABSORPTION & 1.895 & 0.02026 \\
\hline VASOPRESSIN REGULATES RENAL WATER HOMEOSTASIS VIA AQUAPORINS & 1.877 & 0.02040 \\
\hline LYSOSOME VESICLE BIOGENESIS & 1.896 & 0.02043 \\
\hline
\end{tabular}


Supplementary Table S4. (Continued)

\begin{tabular}{|c|c|c|}
\hline Enriched up-regulated pathways & NES & $\begin{array}{l}\text { FDR q- } \\
\text { value }\end{array}$ \\
\hline CALMODULIN INDUCED EVENTS & 1.892 & 0.02051 \\
\hline BIOC CHREBPPATHWAY & 1.898 & 0.02056 \\
\hline CA DEPENDENT EVENTS & 1.900 & 0.02085 \\
\hline THE CITRIC ACID TCA CYCLE AND RESPIRATORY ELECTRON TRANSPORT & 1.900 & 0.02152 \\
\hline WP401 MITOCHONDRIAL LC FATTY ACID BETA OXIDATION & 1.870 & 0.02157 \\
\hline MITOTIC PROPHASE & 1.859 & 0.02232 \\
\hline $\begin{array}{l}\text { SYNTHESIS AND INTERCONVERSION OF NUCLEOTIDE DI AND } \\
\text { TRIPHOSPHATES }\end{array}$ & 1.860 & 0.02243 \\
\hline TRANSLOCATION OF GLUT4 TO THE PLASMA MEMBRANE & 1.864 & 0.02248 \\
\hline DARPP 32 EVENTS & 1.855 & 0.02260 \\
\hline NUCLEAR EVENTS KINASE AND TRANSCRIPTION FACTOR ACTIVATION & 1.850 & 0.02261 \\
\hline $\begin{array}{l}\text { LATENT INFECTION OF HOMO SAPIENS WITH MYCOBACTERIUM } \\
\text { TUBERCULOSIS }\end{array}$ & 1.851 & 0.02286 \\
\hline KEGG BIOSYNTHESIS OF UNSATURATED FATTY ACIDS & 1.860 & 0.02289 \\
\hline INSULIN RECEPTOR RECYCLING & 1.842 & 0.02434 \\
\hline KEGG AMINO SUGAR AND NUCLEOTIDE SUGAR METABOLISM & 1.842 & 0.02471 \\
\hline GLUCAGON TYPE LIGAND RECEPTORS & 1.833 & 0.02616 \\
\hline KEGG COLLECTING DUCT ACID SECRETION & 1.828 & 0.02650 \\
\hline GLYCEROPHOSPHOLIPID BIOSYNTHESIS & 1.822 & 0.02679 \\
\hline KEGG OOCYTE MEIOSIS & 1.829 & 0.02688 \\
\hline APOPTOTIC CLEAVAGE OF CELLULAR PROTEINS & 1.823 & 0.02724 \\
\hline CITRIC ACID CYCLE TCA CYCLE & 1.813 & 0.02917 \\
\hline GLYCOSPHINGOLIPID METABOLISM & 1.794 & 0.03413 \\
\hline INTRINSIC PATHWAY FOR APOPTOSIS & 1.790 & 0.03454 \\
\hline KEGG ALCOHOLISM & 1.779 & 0.03656 \\
\hline BIOC BIOPEPTIDESPATHWAY & 1.780 & 0.03688 \\
\hline TRANS GOLGI NETWORK VESICLE BUDDING & 1.781 & 0.03706 \\
\hline WP2087 MIRNA REGULATION OF DNA DAMAGE RESPONSE & 1.782 & 0.03737 \\
\hline FATTY ACID TRIACYLGLYCEROL AND KETONE BODY METABOLISM & 1.774 & 0.03805 \\
\hline G ALPHA Z SIGNALLING EVENTS & 1.768 & 0.03839 \\
\hline WP317 GLYCOGEN METABOLISM & 1.769 & 0.03877 \\
\hline KEGG PARKINSON S DISEASE & 1.769 & 0.03938 \\
\hline METABOLISM OF NUCLEOTIDES & 1.763 & 0.03947 \\
\hline ADP SIGNALLING THROUGH P2Y PURINOCEPTOR 12 & 1.759 & 0.04059 \\
\hline CLATHRIN DERIVED VESICLE BUDDING & 1.752 & 0.04113 \\
\hline BIOC CREBPATHWAY & 1.755 & 0.04113 \\
\hline KEGG DOPAMINERGIC SYNAPSE & 1.750 & 0.04146 \\
\hline ACTIVATION OF BAD AND TRANSLOCATION TO MITOCHONDRIA & 1.752 & 0.04172 \\
\hline
\end{tabular}


Supplementary Table S4. (Continued)

Enriched up-regulated pathways

NES FDR qvalue

KEGG TIGHT JUNCTION

1.746

0.04235

KEGG RETINOL METABOLISM

SEMA3A PAK DEPENDENT AXON REPULSION

$1.736 \quad 0.04585$

$1.726 \quad 0.04880$

POST CHAPERONIN TUBULIN FOLDING PATHWAY

$1.727 \quad 0.04916$

Supplementary Table S5. List of the significantly enriched down-regulated pathways in NAFLD (FDR q-value $<0.05$ )

\begin{tabular}{l|ll} 
Enriched down-regulated pathways & NES & $\begin{array}{c}\text { FDR q- } \\
\text { value }\end{array}$ \\
\hline WP449 COMPLEMENT AND COAGULATION CASCADES & -2.496 & 0.00000 \\
KEGG COMPLEMENT AND COAGULATION CASCADES & -2.496 & 0.00000 \\
KEGG SELENOCOMPOUND METABOLISM & -2.229 & 0.00018 \\
FORMATION OF FIBRIN CLOT CLOTTING CASCADE & -2.272 & 0.00024 \\
WP20O COMPLEMENT ACTIVATION CLASSICAL PATHWAY & -2.219 & 0.00028 \\
BIOC INTRINSICPATHWAY & -2.081 & 0.00213 \\
COMMON PATHWAY & -2.086 & 0.00226 \\
REGULATION OF COMPLEMENT CASCADE & -2.066 & 0.00304 \\
COMPLEMENT CASCADE & -2.023 & 0.00428 \\
AMINO ACID TRANSPORT ACROSS THE PLASMA MEMBRANE & -1.995 & 0.00606 \\
WP46O BLOOD CLOTTING CASCADE & -1.962 & 0.00857 \\
INTRINSIC PATHWAY & -1.963 & 0.00928 \\
GLUTAMATE NEUROTRANSMITTER RELEASE CYCLE & -1.930 & 0.01245 \\
BMAL1 CLOCK NPAS2 ACTIVATES CIRCADIAN GENE EXPRESSION & -1.832 & 0.04132 \\
SYNTHESIS OF BILE ACIDS AND BILE SALTS VIA 7ALPHA & -1.821 & 0.04408 \\
HYDROXYCHOLESTEROL & -1.806 & 0.04612 \\
WP31O MRNA PROCESSING & -1.806 & 0.04900 \\
KEGG PROTEIN EXPORT & &
\end{tabular}


Supplementary Table S6. The list of genes in core enrichment of NRF2 targets

\begin{tabular}{rl|c}
\multicolumn{2}{c}{ Genes } & Rank in list \\
\hline 1 & NQ01 & 24 \\
2 & UGDH & 36 \\
3 & SRXN1 & 44 \\
4 & GPX1 & 60 \\
5 & ABCB1A & 99 \\
6 & ALDH1A7 & 112 \\
7 & SULT1C2 & 128 \\
8 & GCLC & 141 \\
9 & FTH1 & 151 \\
10 & EPHX1 & 164 \\
11 & GSTM3 & 287 \\
12 & GSTM4 & 332 \\
13 & ALDH3A2 & 344 \\
14 & PGD & 347 \\
15 & SQSTM1 & 387 \\
16 & ALDH1A1 & 508 \\
17 & CES1G & 689 \\
18 & GSTM1 & 730 \\
19 & GSTM5 & 767 \\
20 & CBR3 & 818 \\
21 & ABCC4 & 864 \\
22 & TKT & 899 \\
23 & ALDH9A1 & 910 \\
24 & BLVRB & 1076 \\
25 & GSTM2 & 1115 \\
26 & GPX3 & 1527 \\
27 & GSS & 1726 \\
& & \\
& &
\end{tabular}


Supplementary Table S7. The list of genes in core enrichment of PPARa targets

\begin{tabular}{|c|c|c|c|c|c|}
\hline & Genes & Rank in list & & Genes & Rank in list \\
\hline 1 & CIDEA & 0 & 42 & HMGCL & 1074 \\
\hline 2 & UGT1A9 & 1 & 43 & ACADM & 1096 \\
\hline 3 & CIDEC & 2 & 44 & АСОТ9 & 1144 \\
\hline 4 & MOGAT1 & 9 & 45 & ELOVL5 & 1147 \\
\hline 5 & PLIN4 & 27 & 46 & РСTP & 1178 \\
\hline 6 & $\mathrm{CD} 36$ & 35 & 47 & CYP3A11 & 1220 \\
\hline 7 & $\mathrm{ECH} 1$ & 38 & 48 & EHHADH & 1251 \\
\hline 8 & CPT1B & 55 & 49 & HSD17B10 & 1307 \\
\hline 9 & AQP7 & 56 & 50 & ACAT1 & 1309 \\
\hline 10 & SLC27A4 & 73 & 51 & ACAA1B & 1397 \\
\hline 11 & АСОТ2 & 75 & 52 & SLC25A20 & 1458 \\
\hline 12 & CYP4A14 & 86 & 53 & ECl1 & 1490 \\
\hline 13 & PEX11A & 110 & 54 & ACOT5 & 1501 \\
\hline 14 & PLIN2 & 139 & 55 & АСОT8 & 1507 \\
\hline 15 & VLDLR & 173 & 56 & ACACB & 1521 \\
\hline 16 & CRAT & 244 & 57 & CPT2 & 1543 \\
\hline 17 & CYP4A10 & 306 & 58 & GPAM & 1592 \\
\hline 18 & ALDH3A2 & 344 & 59 & AGPAT3 & 1671 \\
\hline 19 & AGXT2 & 360 & 60 & $\mathrm{HADH}$ & 1683 \\
\hline 20 & ELOVL7 & 410 & 61 & ABCD2 & 1809 \\
\hline 21 & LIPA & 428 & 62 & CYP4A12A & 1826 \\
\hline 22 & HADHA & 449 & 63 & АСОT1 & 1989 \\
\hline 23 & ACAD9 & 509 & 64 & UCP3 & 2131 \\
\hline 24 & IL1RN & 525 & 65 & ACSM3 & 2132 \\
\hline 25 & FABP2 & 541 & 66 & SCD2 & 2139 \\
\hline 26 & ODC1 & 549 & 67 & PLTP & 2292 \\
\hline 27 & SLC25A 10 & 634 & 68 & OAT & 2367 \\
\hline 28 & CROT & 687 & 69 & CPT1A & 2517 \\
\hline 29 & CESIG & 689 & 70 & АСОT7 & 2553 \\
\hline 30 & FGF21 & 699 & 71 & ACOX1 & 2576 \\
\hline 31 & PDK4 & 713 & 72 & CYP2J6 & 2601 \\
\hline 32 & LIPE & 716 & 73 & GPD2 & 2629 \\
\hline 33 & АСОТЗ & 738 & 74 & ACADL & 2813 \\
\hline 34 & GYK & 776 & 75 & HSD17B4 & 2842 \\
\hline 35 & ALDH9A1 & 910 & 76 & MGLL & 2994 \\
\hline 36 & ACOT4 & 916 & 77 & ETFDH & 2997 \\
\hline 37 & ACAD10 & 928 & 78 & АСОT10 & 3011 \\
\hline 38 & RAB9 & 1033 & 79 & ABCB4 & 3036 \\
\hline 39 & DECR2 & 1050 & 80 & UCP2 & 3040 \\
\hline 40 & DECR1 & 1055 & 81 & ACAA1A & 3110 \\
\hline 41 & TXNIP & 1065 & & & \\
\hline
\end{tabular}


Supplementary Table S8. The list of genes in core enrichment of MAPK targets

\begin{tabular}{cl|c}
\multicolumn{1}{c}{ Genes } & Rank in list \\
\hline 1 & PPP2R1B & 12 \\
2 & DUSP3 & 115 \\
3 & MAPK3 & 621 \\
4 & JUN & 1001 \\
5 & MAPK9 & 1066 \\
6 & MAPKAPK2 & 1214 \\
7 & RPS6KA1 & 1407 \\
8 & MAPK8 & 1570 \\
9 & MAPK10 & 2011 \\
10 & PPP2R1A & 2102 \\
11 & PPP2CA & 2797
\end{tabular}




\section{SUPPLEMENTARY FIGURES}

\section{Supplementary Figure S1}

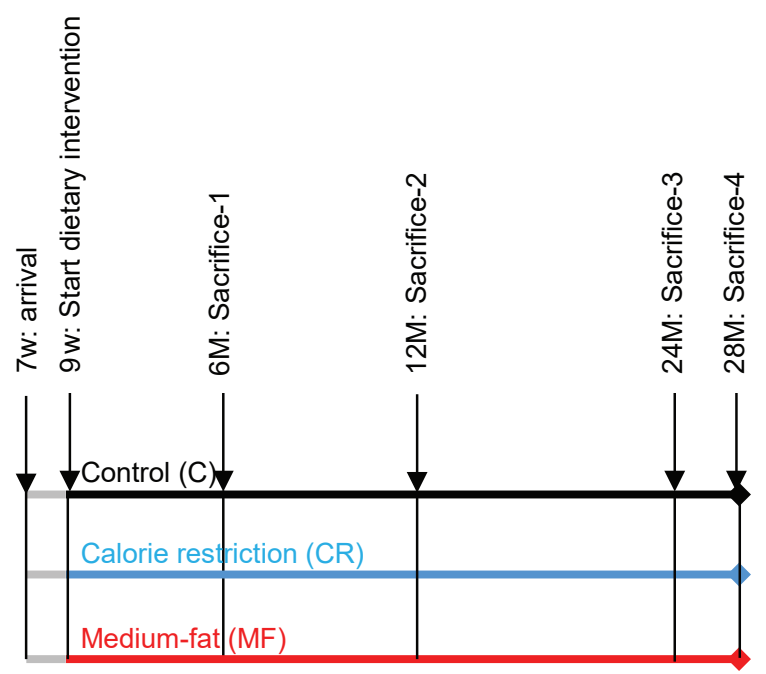

Supplementary Figure S1. Study design scheme. The male C57BL/6J mice arrived at 7 weeks old and were acclimatized for 2 weeks. The dietary intervention was started at the age of 9 weeks and the mice were culled at 6,12 , 24 and 28 months, in order to cover different life stages. 


\section{Supplementary Figure $\mathbf{S 2}$}

A

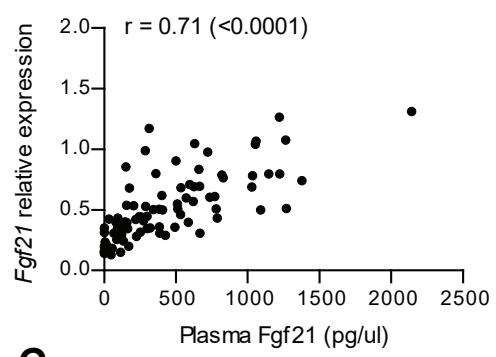

C

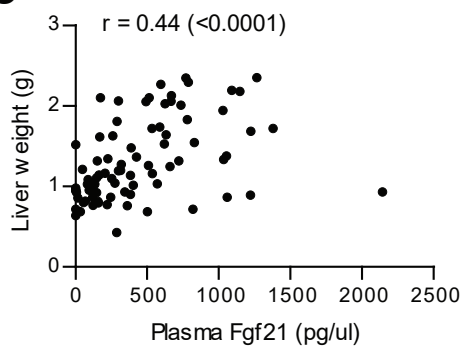

B

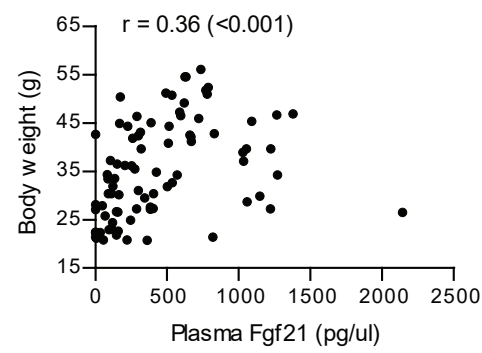

Supplementary Figure S2. (A) Validating the microarray data, plasma Fgf21 levels were positively correlated with hepatic Fgf21 expressions, which was obtained through Q-PCR technique. (B) Plasma Fgf21 levels were positively correlated with body weight. (C) Plasma Fgf21 levels were positively correlated with liver weight. $r$ value and its significance were calculated with Pearson's correlation.

\section{Supplementary Figure S3}

A

ROC curve for all time points

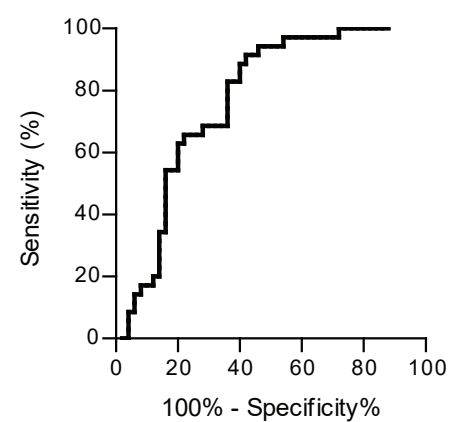

B

ROC curve for $12,24,28 \mathrm{M}$

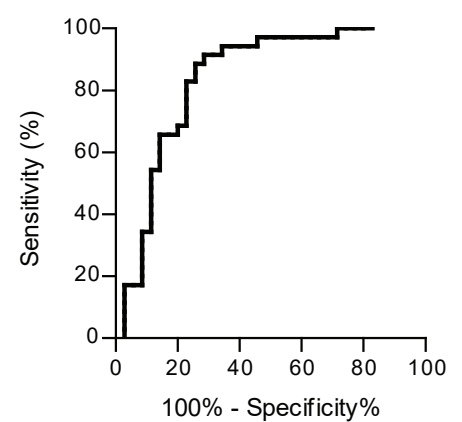

Supplementary Figure S3. (A) ROC analysis of plasma Fgf21 predicting animals with and without NAFLD. In this analysis, all animals from 4 age time points were included. (B) ROC analysis of plasma Fgf21 predicting animals with and without NAFLD at middle and old age. The 6 month-old mice were excluded in this analysis. 


\section{Supplementary Figure S4}
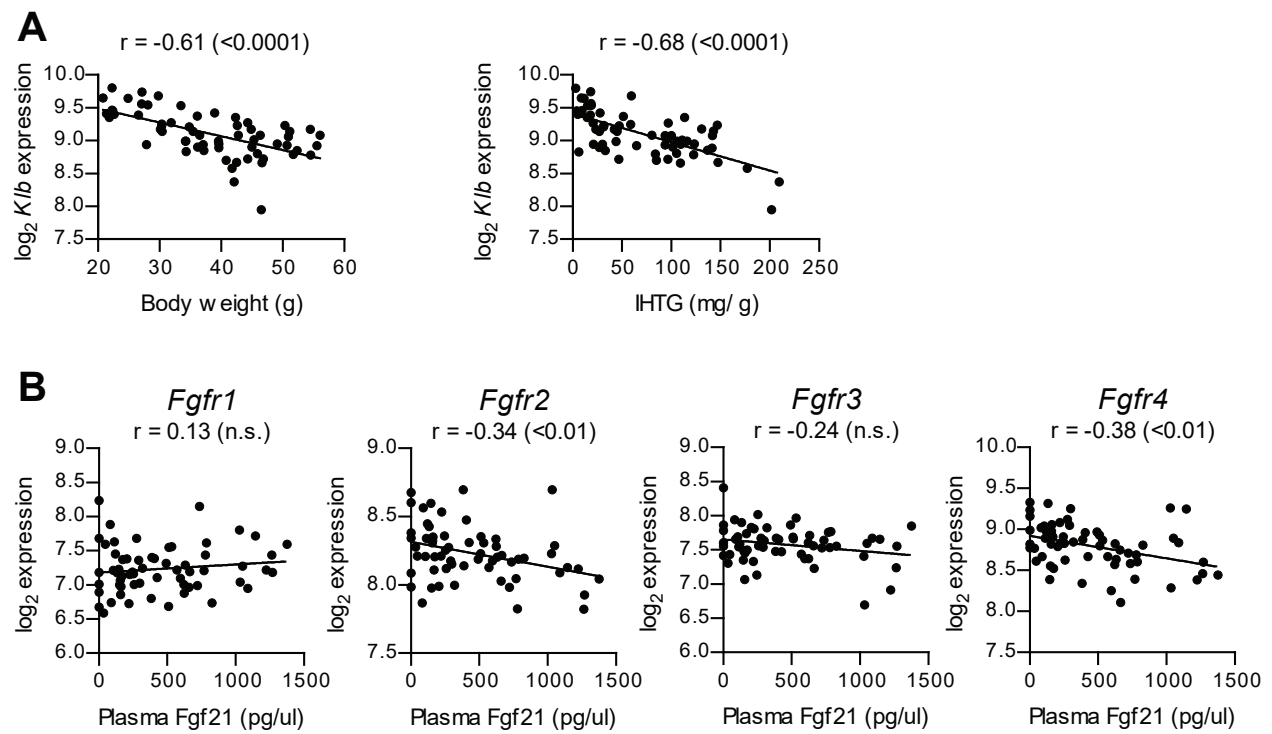

Supplementary Figure S4. (A) Body weight and intrahepatic triglyceride (IHTG) were inversely correlated with $\mathrm{Klb}$ expression levels. (B) Correlations between the plasma Fgf21 and gene expression levels of Fgfr1, Fgfr2, Fgfr3, and Fgfr4. $r$ values and their significance (in parentheses) were calculated with Pearson's correlation. 


\section{Supplementary Figure S5}

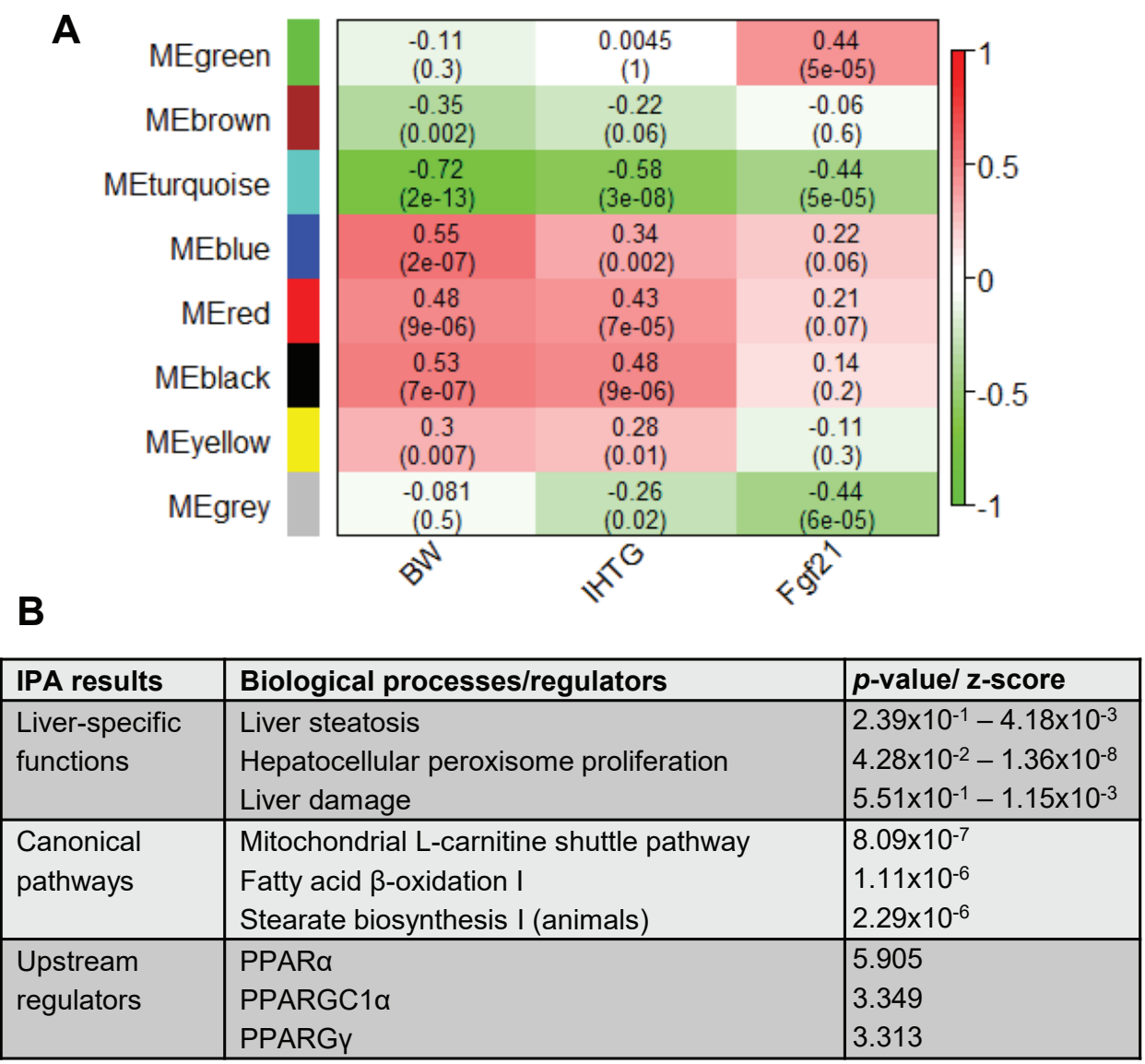

Supplementary Figure S5. (A) Heat map depicting the correlation between gene modules (in rows) and phenotypes (in columns). To investigate the functions of the genes associated with the elevated Fgf21 plasma level at young age without accumulation of IHTG, this analysis was performed with the inclusion of the 6 month old animals. In this analysis, the variable deepSplit setting in WGCNA was fine-tuned to obtain a module with a strong correlation with plasma Fgf21 levels, but not with IHTG content. The top values in each cell represents the correlation coefficient between the module and phenotype with the correlation $p$-value in parentheses. Red and green color represents positive and negative correlation, respectively. (B) Biological processes, pathways and regulators associated with MEgreen (in Supplementary Figure S5A). Significant liver-specific functions and canonical pathways are reported in $p$-values. Significant upstream regulators are reported in predicted activation z-score. Positive and negative z-score represent predicted activation and inhibition, respectively. 




\section{Significance of this study}

The study presented in this thesis adds to our understanding the important impacts of different diets have on genotype-phenotype relationships, which translate into different health states. We investigated the effects of different dietary regimen on the phenotypes of genetically identical mice, particularly of an intermittent (INT) diet, which alternates weekly between the ad libitum mediumfat (MF) and calorie restriction (CR) diet. The research largely focused on the liver as the central organ in metabolic regulation and aging markedly alters its morphology, physiology and capacity to function. This is reflected by the high prevalence of non-alcoholic fatty liver disease (NAFLD) in the elderly population. We found that the repetitive bouts of CR diet within the INT dietary regimen protected the liver from developing advanced stages of NAFLD, which was induced by exposure to the MF diet (chapter 2 and 3). We further examined the potential of the INT diet as a treatment to counteract the effects of a long-term continuous MF diet and revealed that, important metabolic features (glucose clearance, liver health and survival) were successfully improved, despite the development of hepatic steatosis (chapter 3). By switching the diets at a defined time point during the study (chapter 3 and 4), we demonstrated that, even at middle and old age, the liver is still a highly flexible organ that rapidly adapts its transcriptional program to the different dietary challenges. In the last chapter, we explored the diet-induced development of NAFLD during aging. We also demonstrated that the strong link between the diet-induced NAFLD and fibroblast growth factor (Fgf21) denoted a dysregulation of PPARa signalling pathway during the development of the liver disease (chapter 5).

\section{Intermittent calorie intake as a novel healthy dietary regimen?}

The key physiological profile of INT-exposed animals during aging is summarized in Figure 1, whereby the degree of similarity of INT-exposed animals at middle and old age, compared to the continuous CR and MF diets is depicted. At both 12 and 24 months of age, the body weights of INTexposed animals were between those of CR- and MF-fed animals, as might be expected from the overall energy intake. With aging, plasma insulin and IL-6 of animals in the INT diet group became more comparable with the profiles of the CR group. Furthermore, unlike in the continuous MFexposed animals, NAFLD in the INT diet group did not progress to severe pathology, as indicated by the liver 4-hydroxyproline content and plasma ALT levels that are similar to those of CR group. In contrast, intrahepatic triglyceride (IHTG) substantially elevated during aging (see also Figure 2), reaching a level similar to that of the MF group at the age of 24 months. It is important to note that these measurements were performed consistently during the ad libitum MF feeding week and to draw a more definitive conclusion, ideally, measurements during the restricted feeding week 
should have been included. Despite this limitation, the data still strongly suggests that there was an age-related alteration in the INT diet group that led to the development of hepatic steatosis. At the gene expression levels (chapter 3), although the transcriptomic profile of the INT-exposed mice at the middle age time point resembles that of the MF group, at the old age of 24 months the gene expression profile of INT shifts toward the profile of CR group. Thus, weekly intermittent calorie restriction largely reversed the long-term health effects caused by the lipogenic MF diet.

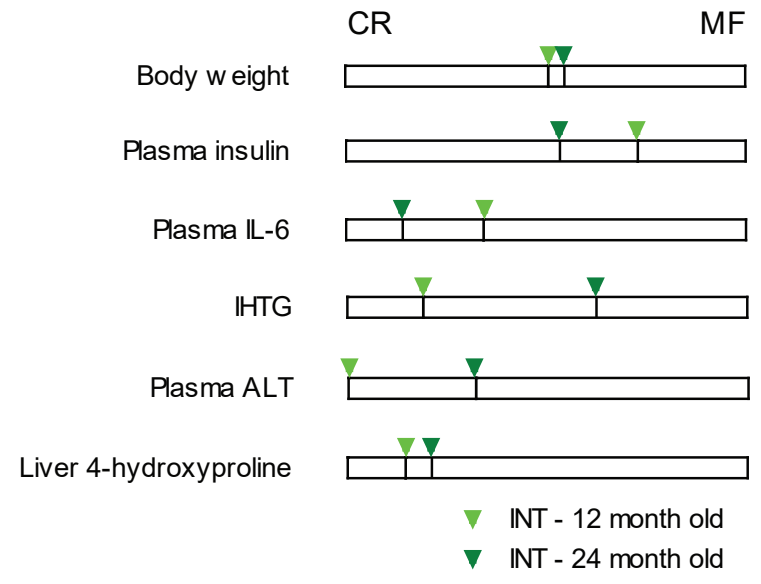

Figure 1. Feature similarity of the INT diet group at 12 and 24 months in comparison to the CR and MF groups. The bar represents the difference between the CR and MF groups, which was set as $100 \%$ for each timepoint. Then, the position of the INT-exposed group within the $100 \%$ scale was determined and represented by the line and pointer.

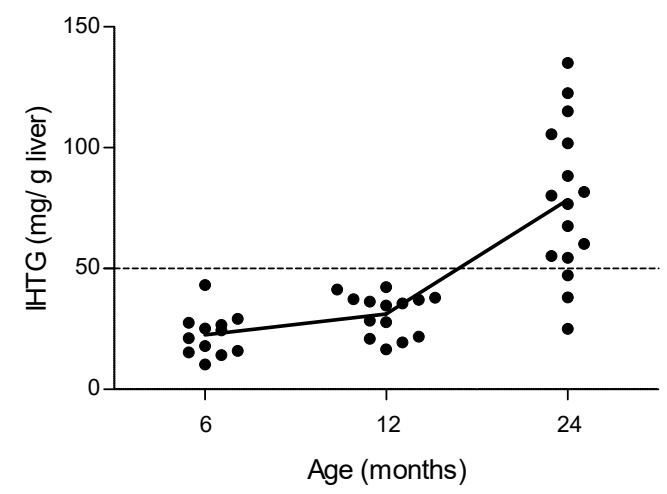

Figure 2. The intrahepatic triglyceride (IHTG) content of the INT-exposed animals at different time points. Dashed line indicate the commonly applied threshold to define NAFLD, $5 \%$ or $50 \mathrm{mg}$ triglyceride per $\mathrm{g}$ liver [1]. 
We hypothesized that the INT dietary regimen might achieve its beneficial effects in counteracting the adverse effects of the MF diet through the repeated exposure to metabolic challenges during the restricted feeding week, i.e. stress from energy deficiency and time-restricted feeding [2-4]. The compelling weekly fluctuation of $\sim 15 \%$ body weight indicates that metabolism was strongly challenged in this dietary regimen. At the final sacrifice ( 28 months) we had the opportunity to include an extra group of INT-exposed animals culled during the restricted feeding week. By comparing the physiological features measured following ad libitum MF and restricted feeding week, we confirm that the weekly alternating regimen induced strong physiological changes (Figure $\mathbf{3}$ ). In addition to the body weight difference, there was a pronounced alteration in the visceral white adipose tissue (WAT) weight, but not in the epidydimal WAT. This is an intriguing observation regarding the partitioning of fat deposition, suggesting that during the weekly fluctuation of energy availability, the visceral fat depot was more flexible than the more stable epidydimal one. Visceral WAT dysfunction is thought to be implicated in obesity and insulin resistance and likely plays a role in NAFLD [5]. It has been suggested that the proximity of visceral WAT to the portal vein may allow visceral WAT to exert more direct metabolic effects on the liver [6].
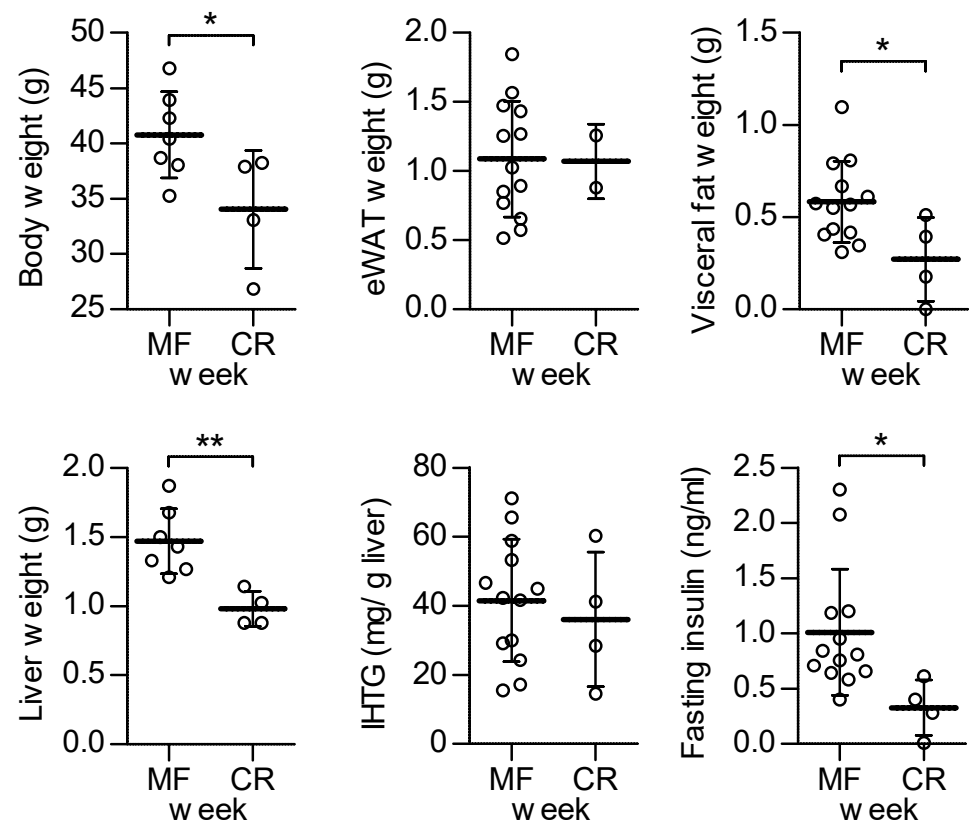

Figure 3. Physiological alterations in the ad libitum MF and restricted feeding week of the INT dietary regimen, which was measured at the age of 28 months. Error bars represent standard deviation. *) $\left.p<0.05 ;{ }^{* *}\right) p<0.01$ 
Liver weights measurements also showed a difference between the ad libitum and restricted feeding week (Figure 3). However, despite the difference in liver weight, the analysis of liver triglyceride content revealed that IHTG was not significantly altered. Possible explanations are 1) IHTG content is expressed as a concentration, which denotes that the absolute amount of IHTG would still be substantially higher in the MF week, 2) at a very old age, the redistribution of IHTG in the CR week (that we hypothesized in chapter 2) was not effective anymore, or 3 ) it is possible that the fluctuation occurred on other liver constituents, such as glycogen. This intriguing observation merits a further research, since this will bring insight on how dietary intervention affect lipid deposition in different organs over the course of time: does the fat storage partitioning and/or fuel utilization following a metabolic challenge alter during aging? To investigate this, multiple age time point comparison for both ad libitum MF and restricted feeding week have to be used/performed.

The daily activity measurement revealed an interesting finding that the INT-exposed animals showed a spontaneous increase of daily activity to a level comparable to that of the CR group (chapter 3). Although this was thought to occur only in the restricted feeding week due to foraging activity when hungry, unexpectedly we found the same enhanced level of activity in the ad libitum feeding week. The increased activity level will most likely contribute substantially to the overall health advantages of the INT diet over the MF diet, in addition to the diet effect itself. Therefore, this study provides novel insights of 1 ) the INT diet-induced effects on increasing physical activity and 2) the importance of performing behaviour or physical activity measurements animal studies exploring novel dietary intervention. In human studies, dietary intervention and physical activity modification are two independent variables; physical activity in human has to be modified intentionally.

Due to the complexity and extensiveness of this mice aging study, we were not yet able to explore all interesting and promising aspects of the INT-induced alterations. We have not yet investigated the status of signalling pathways and transcriptional effectors responding to energy stress, such as the reduced insulin/insulin-like growth factor (IGF-1) signalling, the target of rapamycin (TOR) signalling repression, and AMP-activated protein kinase (AMPK) activation [7]. The cycles of CR and ad libitum MF feeding in INT dietary regimen can also be expected to cause fluctuations of various intermediate metabolites, such as acetyl-CoA, uridine diphosphate-glucose, a-ketoglutarate, nicotinamide adenine dinucleotide ( $N A D^{+}$), flavin adenine dinucleotide [8]. These metabolites are utilized by chromatin-modifying enzymes and other transcriptional regulators $[8,9]$, which may significantly affect chromatin activity. Therefore, the challenge imposed by INT dietary regimen would also likely induce some features of 'chromatin exercise' [10], affecting posttranslational modifications of histones and DNA and, in the long run, maintain chromatin flexibility.

The INT regimen imposes the effects from both calorie and feeding time restriction. When 
the mice received their daily rations during the restricted feeding week, they ate immediately and stayed fasted until the next feeding time. Time-restricted feeding without reduction in calorie intake has been shown to be beneficial for health by improving nutrient sensing pathways and preventing from developing metabolic disorders induced by high-fat diet [11]. This brings about the question of, between the calorie reduction and the time-restricted feeding in the INT dietary regimen, which one has the compelling effect mediating the beneficial effects of INT dietary regimen? To investigate this, a comparison with an isocaloric pair-fed group would be required. When the eating pattern is kept constant, time restriction factor can be excluded and this allows to examine which factor is essential in mediating the beneficial effects of INT diet.

The alternating dietary pattern between $\mathrm{CR}$ and MF diet is also expected to have an immediate effect on the gut microbiota composition. Studies have reported microbiome alterations, e.g. the increased proportion of Firmicutes to Bacteroidetes, in obese subjects [12-14] and diet-induced mice $[15,16]$. Although not a direct measurement, urine metabolomics analysis that was performed on the 28-month-old animals provided indications that microbiota composition changed rapidly in response to the weekly alternating dietary regimen. Urine samples of the INT-exposed animals were collected for both the restricted and ad libitum feeding week. Metabolites that are related to microbial activity are $p$-cresol and trimethylamine (TMA). The levels of p-cresol and TMA showed to fluctuate according to the feeding week. However, while the fluctuation pattern of $p$-cresol was similar to the continuous CR or MF diet, the elevated level of TMA in the ad libitum MF feeding week was significantly different from the continuous MF diet group (Figure 4). P-cresol is derived from tyrosine in reactions involving gut bacteria, i.e. some species of Clostridia [17] and TMA is a product of bacterial degradation from choline in distal intestine [18]. Thus, this analysis of the urine metabolite provides an indication that microbiota composition could be altered rapidly within a week of alternating diet. It would be interesting to investigate further how the microbiota population in the different parts of the small intestine and the colon adjust to the INT diet and what are the systemic and liver-specific metabolic consequences. 

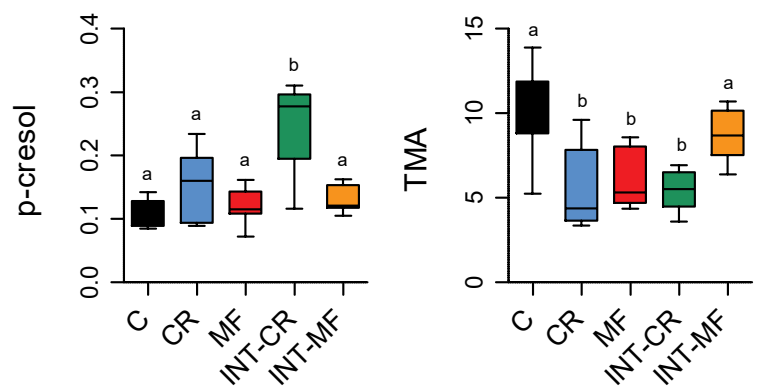

Figure 4. The levels of $p$-cresol and trimethylamine (TMA) measured in the mice urine samples at the age of 27 months, by using ${ }^{1} \mathrm{H}-\mathrm{NMR}$ measurement technique [19]. Urine samples collection covered both feeding weeks in the INT dietary regimen.

The type of control diet used in this study is a semisynthetic diet, which is rich in easily digestible carbohydrates (76E\% carbohydrate, composed of $62 \%$ corn starch, $14 \%$ maltodextrin, $14 \%$ sucrose and $10 \%$ dextrose). We have used these diets already for many years [20] in most of our animal studies because of the well-defined composition compared to ill-defined chow diets that are still widely used. We observed that also control mice (low-fat) developed overweight and a number of their physiological profiles, i.e. IHTG content, were comparable to those of MF-exposed mice. Benoit et al. (2013) compared chow-low fat diet (LFD) and semisynthetic-LFD to semisynthetichigh fat diet (HFD) and discovered that conclusions on body weight gain, insulin sensitivity and adipose tissue inflammation were dependent on the type of the LFD control [21]. Microbiota analysis of small intestines collected from the current study revealed that diet rich in easy digestible carbohydrates increased the ratio of Firmicutes to Bacteroidetes, compared to chow diet containing more dietary fibres (M. Müller, personal communication). Therefore, a better comparison may be obtained by using a purified diet that more closely resembles the composition of chow diet, but without its fluctuation in composition. Sucrose and digestible starch-rich diets have been linked to increased de novo lipogenesis (DNL) and related changes in lipid metabolism that contribute to liver fat accumulation [22, 23]. Fatty liver is a result from an interplay of lipid accumulation (DNL, lipolysis, and dietary lipids) and removal (lipoprotein secretion and fatty acid oxidation) [24]. Carbohydrate excess has double takes on this interplay: 1) direct effect of increased DNL in order to store the excess carbohydrate as triglycerides, 2) an intermediate in DNL, malonyl-CoA, acts as an inhibitor of carnitine palmitoyl transferase-1, which results in $\beta$-oxidation restriction [25].

To conclude, although the health features of INT diet were not as superior as those of the continuous CR diet, we found that the INT dietary regimen provided a remarkable protection against the severe health outcomes of the long-term MF diet consumption. The repeated exposure to metabolic challenges during the restricted feeding period likely contributed to the significant health improvements of INT dietary regimen. 


\section{Development of hepatic steatosis in the INT-exposed mice at old age: a loss of plasticity?}

In chapter 2 we proposed that, due to exposure to the INT regimen, liver fat can be effectively reduced during the restricted-feeding week. This is due to the IHTG content of INT-fed animals that strongly differed from those of MF diet group, even when the measurement was performed after their MF feeding week. However, to our surprise at older age this effect was less pronounced (see Figure 2). This suggests that the metabolic capacity required for the modulation of liver fat content by catabolism and redistribution at old age might be impaired. A number of studies, which have reported dysregulation of fat metabolism during aging, indicated a similar notion. Houtkooper and co-workers reported the dysregulation of fat metabolism at old age indicated by the lipidomic profiles [26]. Furthermore, it has been demonstrated in the liver that, although the mitochondrial activity did not decrease, the function/efficacy of the electron transport chain decreases with aging. Therefore, the increased mitochondrial activity and $\beta$-oxidation do not necessarily reflect an efficient electron transport chain [27-29]. The authors also found that the electron transport chain in animals with fatty liver is inefficient. Therefore, it is worthwhile noting that, despite the up-regulated fatty acid oxidation in both young animals without NAFLD and old animals with NAFLD, the up-regulation in old animals with NAFLD measurement might lead to perturbing consequences, such as hepatic oxidative stress.

In terms of loss of liver plasticity and function during aging, it would be interesting to zoom in to how exactly the plasticity/function was lost between the age of 12 and 24 months, in order to learn when exactly the shift of metabolic health occurs and why. We proposed that the shift might occur in two different ways. Inspired by a review article by Muoio [30], we use the mitochondrial energy selection as an example of metabolic plasticity. Figure 5A and $\mathbf{B}$ depict how energy homeostasis is maintained by switching between fuels suitable with the energy status. In the energy surplus condition, carbohydrate is the main source for energy combustion, while during a state of energy stress, lipid metabolism is activated and fatty acid oxidation provides energy to most types of tissues. The loss of plasticity during aging that we observed here could manifest in two ways. The first possibility involves a "hit", which impact will impair the homeostasis (Figure 5A). Such a hit could be a damaging environmental stimuli, a set point or a threshold. As an example, when the liver fat content exceeds a threshold for a certain lipid species, it results on lipotoxicity, which impairs the lipid homeostasis [31]. In the second model involves a gradual loss of homeostasis (Figure 5B), which could be illustrated by the progressive decreasing levels of growth hormone and IGF-1 with age [32, 33]. 
A

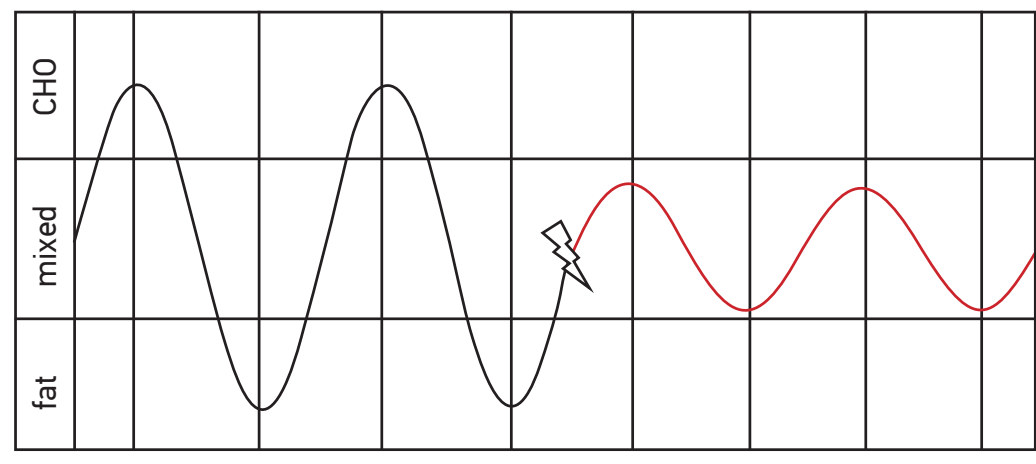

$+$

$+$

$+$

Energy availability

B
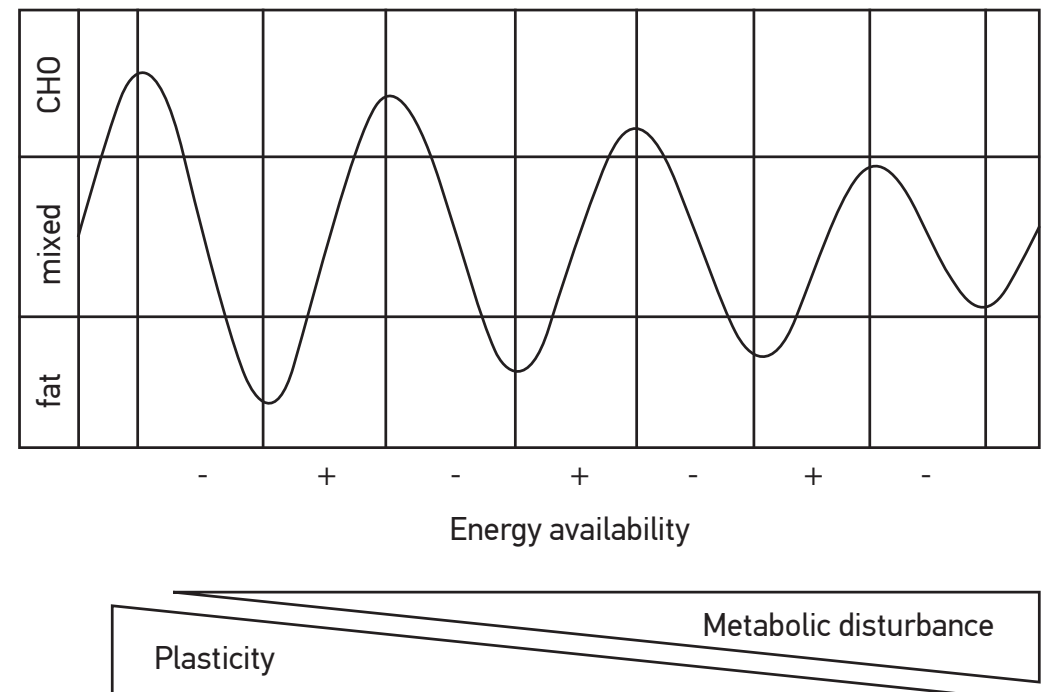

Figure 5. A conceptual model of the loss of metabolic plasticity during aging. In healthy, metabolically flexible state, in a high energy availability condition, carbohydrate (glucose) is the oxidative fuel used, while, in low energy availability, such as in post-absorptive state or fasting, fat oxidation will be the predominant fuel. In between the two states, a mixture between fat and carbohydrate is utilized. When plasticity is lost, the capacity to switch freely between oxidative fuels depending on the nutritional context is lost, represented by persistent oxidation of a mixture of carbon fuels [30]. In the "hit" model (A), plasticity is impaired following damaging environmental stimuli, a set point or a threshold. On the other hand, in the gradual loss model (B), plasticity is progressively impaired. The decreasing plasticity results in increasing metabolic disturbance. The model is an adaptation from Muoio [30]. 
Another factor that possibly contributes to the liver fat accumulation is the microbiota composition. Microbiota composition changes during aging [34-36], and the changes may favour the fat accumulation, for example by increasing the capacity to harvest energy from the diet. This highlights the importance of investigating how multiple tissues/organs are interconnected in relation to the observed metabolic disturbance.

\section{PPARa challenge as a readout of metabolic plasticity}

We initially explored the possibility of utilizing PPARa challenge experiment as a readout to measure plasticity of lipid metabolism. We expected that, due to the metabolic challenge it imposes, the INT dietary regimen would result in a better plasticity at an old age. A treatment of PPARa agonist, Wy-14,643, on a subset of mice from each diet group was performed in every sacrifice time point. However, we discover that there was no significant difference in the regulation of PPARa target genes between animals exposed to different dietary interventions or age time points. Figure 6 displays an example of PPARa target gene response to Wy-14,643 treatment at the age of 24 months. However, when the PPARa response was distinguished between animals with/without NAFLD (chapter 5), a clear difference in PPARa targets' expression levels was observed, particularly for Fgf21, Hmgcr and Mogat1 expression levels. It appears that liver fat is the burden of the liver's plasticity of lipid metabolism. A similar notion was reported by Hyotylainen and co-workers, that high liver fat markedly hampers the ability of the liver to adaptively regulate metabolism to meet the excessive demands on basic liver functions, and therefore, individuals with NAFLD may be more vulnerable to various metabolic stressors on the liver [37]. Our mice cohort exhibited a large inter-individual variation in the development of NAFLD, particularly in the ad libitum control- and MF-fed groups. Therefore, here hepatic steatosis appears to be a stronger determinant for PPARa metabolic plasticity. 

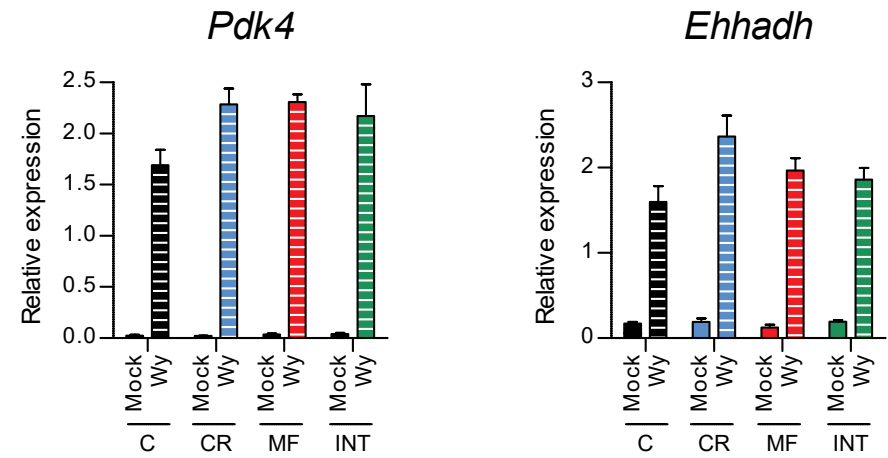

Figure 6. The gene expression levels of PPARa target genes, Pdk4 (left) and Ehhadh (right), when treated with either mock or PPARa agonist Wy-14,643. Gene expression was measured at the age of 24 months and normalized to a housekeeping gene. Colors denote the type of dietary intervention (black = Control, red = MF, green =INT, blue =CR). For each diet group, the measurements of mock- and Wy-14,643-treated animals were performed, which are denoted by $C$ and WY in the graph, respectively. Error bars represent standard deviation.

Such dynamic measurements by using system perturbation or challenge tests are likely more valuable to define metabolic health or resilience, compared to more static measurements [38], e.g. oral glucose tolerance test depicts glucose homeostasis better than one time point measurement of plasma glucose or insulin. Therefore, it is worthwhile to find other challenge tests that are more appropriate to measure the homeostasis capacity, for instance AMPK- $\mathrm{a}_{2}$ activation test by using AICAR or $\beta$-guanidinopropionic acid [39]. The differential response to the two compounds have been shown to be age-related in muscle $[39,40]$. In the current study, the mock and Wy-14,643 treatments of the PPARa challenge were performed from another set of mice within the same diet/ age groups. Considering the heterogeneity of C57BL/6J mice, ideally both treatments should refer to the same mice. This could be performed in experiments by using primary hepatocytes, which would also provide more liver-specific effects, instead of a complex in vivo responses.

\section{Liver plasticity induced by the diet switches}

In chapter 3, the obesity-counteracting effect of the INT diet was investigated. Part of the mice that had been exposed to the MF diet till 12 months of age were transferred to the INT diet until sacrifice at the age of 24 months. The switch to INT diet successfully improved glucose clearance, survival and liver health, although failed to improve IHTG levels. In relation to the proposed models describing how plasticity/function is lost, it is inciting to speculate that the MF-induced irreversible gene(s) might play a role as a set point in the proposed "hit" model (Figure 5A). The persistently regulated lipid metabolism and/or storage genes, i.e. Ppara, Cd36, Cidea, Cidec, Cyp7b1, might "set" the homeostasis to be either inefficient in transporting and oxidizing fatty acid or susceptible for fat 
accumulation.

From this experiment we also learn that, despite the health improvement of INT diet, the risk imposed by the first 12 months of MF diet exposure for development of liver dysfunction persists. This implies that irreversible and potentially serious health risk imposed by Western-style diets may start since early age and an intermittent CR can be a practical way to avoid the health risk. Ubiquitin-conjugating enzyme 2c (Ube2c; Figure 7) is an example of hepatocellular carcinoma gene $[41,42]$ which expression elevated already at 6 month of age in the continuous MF diet, but INT diet appears to reverse this effect. Overexpression of Ube2c leads to loss of genomic stability, since the cells neglect the mitotic spindle checkpoint signals [43].

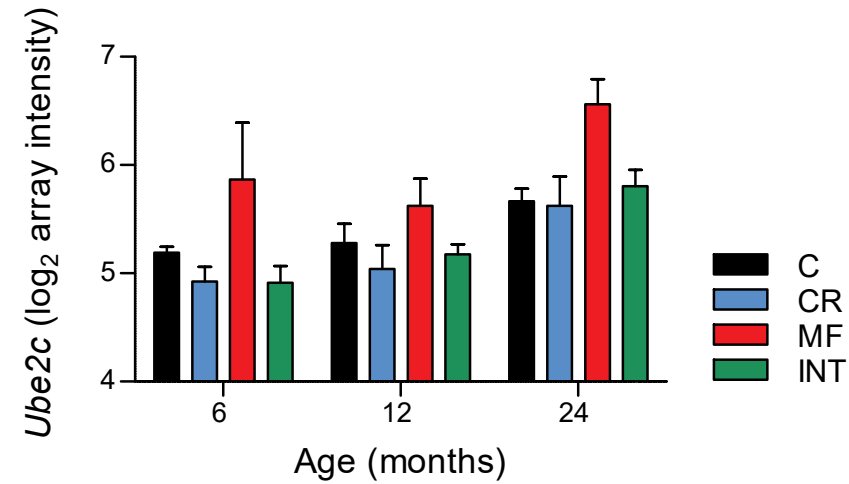

Figure 7. Gene expression levels of Ube2c, a hepatocellular carcinoma-related gene, showing that, starting as early as 6 months of age, the expression levels of the MF diet group were elevated. However, no elevation was observed in the INT and other diet groups.

The second diet switch experiment, although focused on the converse direction from healthy CR diet to the less healthy MF diet, similarly demonstrated the plasticity of metabolism in adapting to the diet switch. This experiment particularly observed that the robust metabolic network that includes multiple regulators contributing its plasticity in coping with the challenge of MF diet, as displayed by the dynamic of $C d 36$ and $C a v 1$ expressions. At the epigenetic level, the diet switch also revealed the plasticity of DNA methylation status.

\section{Relevance to aging and NAFLD development in human population}

Muoio (2014) and Mattson (2014) proposed that the challenge on metabolic flexibility and not leaving the metabolic state at a chronic overnutrition status all the time, are the keys to the health improvement $[4,30]$. Chronic overnutrition leads to a state of metabolic confusion, wherein excessive carbon supply and heightened substrate competition give rise to a set of 
muted and/or conflicting signals [30]. The results obtained from this extensive animal study have shown that the INT dietary regimen largely protected the liver from developing advanced stages of NAFLD. Many variants of this dietary regimen have been explored in both animal models and humans, and, in general, they show promising health improvements. For example, intermittent calorie restriction in humans has been reported to increase insulin sensitivity, even more than daily CR that achieves similar weight loss [44, 45]. Therefore, a repetitive exposure to metabolic stress could be an effective intervention for health improvement. This also implies that, in addition to intervening the food intake that tackles the nutrient surplus, another possibility is the modification on the physical activity intermittently, for example in the "exercise snacking" regimen [46]. Recent studies have established a strong positive association between exercise training and metabolic flexibility $[47,48]$. Or more recently, a nutritional strategy that incorporates both diet and exercise management has been shown to improve metabolic health in older adults [49].

The choice between calorie restriction and fasting as the intervening metabolic challenge is still debated. Fasting imposes greater health risks, especially to subjects who are frail and old, and patients with diabetes receiving insulin or insulin-like drugs. Thus, the lack of medical supervision in subjects undergoing intermittent fasting or alternate-day fasting regimen might result in severe adverse effects. The effect of fasting could be similarly induced in CR, although the less severe intervention of $\mathrm{CR}$ means a longer time required to achieve the effect. The application of fasting mimicking diet (FMD), for instance, similarly induce $\sim 15 \%$ body weight fluctuation as we found in our INT regimen. In FMD regimen, this effect on body weight was induced by 4 days of fasting at $40 \mathrm{E} \%$ restriction [50].

Another notable implication of this finding is the importance of a long-term investigation on the effects of a novel dietary regimen. To our knowledge, only this study and that of Brandhorst et al. (2015) investigate the effects of FMD in a long period that extends to old age (16-30 months) [50]. Most of other animal studies applied the novel diets for only 4-20 weeks [51-53]. Another study of intermittent fasting (IF) regimen has a longer term of 32 weeks, in addition to the comparison of short period of 4 weeks in the same study [54]. While in the Brandhorst study the health markers measured did not show major adverse effect of FMD [50], the study on IF dietary regimen provided a similar notion to our study that, in the long run, IF impairs redox balance and glucose tolerance, but did not do so in the short-term [54].

Another dietary strategy that shows promising health beneficial effects is protein restriction. Protein restriction, which could also be derived from the restriction of a certain amino acid, demonstrated similar effects to $\mathrm{CR}[55,56]$, but without any calorie reduction. One of the 
signalling pathways associated with aging, mTOR, is specifically activated by amino acids, which is the rationale of how protein restriction leads to healthy aging by avoiding mTOR activation. As there are more pathways involved in aging, e.g. insulin/IGF-1 signalling, protein restriction may not encompass all beneficial effects of CR. Furthermore, protein restriction is not compatible at all ages. Older adults may be protected from losing muscle mass by consuming more protein $[57,58]$.

The last alternative to intervene with aging is pharmacological treatment. A number of pharmacological agents for this purpose has been discovered. Compounds derived from plant foods include resveratrol, epicathechin, curcumin and sulforaphane, while the pharmacological alternatives cover rapamycin, rimonabant, metformin and AICAR $[59,60]$. The application of these agents, however, does not completely mimic the effects of CR. Each compound has specific signalling target(s), for example AMPK is the target of AICAR, and AMPK activation in not responsible for all the benefits achieved by CR.

It should be noted that sex dimorphism is an important aspect in response to dietary intervention and aging. Female rats has been shown to display an extreme response to $40 \mathrm{E} \%$ $\mathrm{CR}$ in comparison to the male counterparts [61]. The females became emaciated, ceased menstrual cycling, underwent endocrine masculinization, exhibited changes on behaviour and activity profile. The $40 \mathrm{E} \%$ restricted male mice maintained a higher body weight than the females and did not change their activity levels as significantly as the females. However, the male and female exhibited similar metabolic responses of circulating lipids (cholesterols/ triglycerides) and energy-regulating hormones (insulin, leptin, adiponectin, ghrelin) to the CR [61]. In human, the metabolic responses have been studied in a different approach. General responses to energy stress, such as increases in plasma fatty acid and ketones, and decrease in plasma glucose, do not differ between genders, however gender-related differences on lipid metabolism appeared in a short term CR [62]. Male subjects showed greater propensity to take up and store fatty acid in the liver, compared to the female subjects [62]. The age-related hormonal alteration, i.e. estrogen level pre- and post-menopause [63], also has a large impact on metabolic health, and therefore investigating the diet-induced phenotypic plasticity on female mice would require a separate study dedicated for this purpose.

\section{Final Conclusion and Future Perspectives}

Faced with the fast-growing aging population in a nutrition superfluous environment, researchers in the field of nutrition and health sciences continually strive to find new strategies to support healthy aging. In this thesis we studied the intermittent CR diet as a novel dietary 
regimen for healthy aging and we found that it provided a remarkable protection against the severe health outcomes of the long-term medium-fat diet consumption. We demonstrated that this diet improves life quality by reducing the burden of chronic disease, especially those concerning the liver, providing support for the use of INT diet to promote healthy aging. We demonstrated that the liver is a highly flexible organ metabolically and dietary pattern shapes its metabolic plasticity. These health improvements puts forward the INT diet as a potential health strategy in combating metabolic adverse effects of our modern diet. Moreover, it is intuitively likely that people will find this diet easier to comply to, compared to a continuous CR regimen. Therefore, the intermittent CR represents an attractive area for further investigation.

Currently, it is too early to conclude that the INT dietary regimen (or modulation of the energy intake) would provide the same beneficial effects and is safe to be applied in the human population. However, this study is a proof-of-concept of intervening with a chronic overnutrition status with a metabolic challenge of energy stress. Further investigation on this novel dietary regimen is needed to allow this regimen to be safely applied in humans. To mention the two most important subjects, the first is to investigate the holistic consequences of this regimen on different organs/tissues and also at different molecular levels, i.e. epigenome and microbiome. The second is to determine the extent of the metabolic challenge that is beneficial in humans. This includes the degree and duration of $\mathrm{CR}$, diet quality and modification on physical activity. These are especially crucial, since it is possible that a certain degree of calorie restriction simultaneously benefit some organs/tissue but harm others [64]. The type of test can be used to quantify metabolic health merits further investigation, since we showed here that PPARa challenge exhibited quite a robust response and might not be sensitive enough to reveal the weak point in the metabolic health network.

In sum, the take home message from this study is that the metabolic health during aging is highly modifiable by dietary pattern and the novel intermittent calorie restriction diet provides a remarkable protection against the severe health outcomes of the long-term medium-fat diet, which poses adverse health effects. This diet improves life quality by reducing the burden of chronic disease, and therefore, represents an attractive area for further investigation. 


\section{REFERENCES}

1. Kleiner, D.E., Brunt, E.M., Van Natta, M., Behling, C., et al., Design and validation of a histological scoring system for nonalcoholic fatty liver disease. Hepatology, 2005, 41,1313-1321.

2. Calabrese, E.J. and Baldwin, L.A., Defining hormesis. Human \& Experimental Toxicology, 2002, 21,91-97.

3. Mattson, M.P., Hormesis defined. Ageing Research Reviews, 2008, 7,1-7.

4. Mattson, M.P., Challenging Oneself Intermittently to Improve Health. Dose-Response, 2014, 12.

5. Tiniakos, D.G., Vos, M.B., and Brunt, E.M., Nonalcoholic Fatty Liver Disease: Pathology and Pathogenesis. Annual Review of Pathology: Mechanisms of Disease, 2010, 5,145-171.

6. Virtue, S. and Vidal-Puig, A., It's Not How Fat You Are, It's What You Do with It That Counts. PLoS Biol, 2008, 6,e237.

7. Anderson, R.M. and Weindruch, R., Metabolic reprogramming, caloric restriction and aging. Trends in Endocrinology \& Metabolism, 2010, 21,134-141.

8. Gut, P. and Verdin, E., The nexus of chromatin regulation and intermediary metabolism. Nature, 2013, 502,489-498.

9. Bell, O., Tiwari, V.K., Thomä, N.H., and Schübeler, D., Determinants and dynamics of genome accessibility. $2011,12,554-564$

10. Vaquero, A. and Reinberg, D., Calorie restriction and the exercise of chromatin. Genes \& Development, 2009, 23,1849-1869.

11. Hatori, M., Vollmers, C., Zarrinpar, A., DiTacchio, L., et al., Time-Restricted Feeding without Reducing Caloric Intake Prevents Metabolic Diseases in Mice Fed a High-Fat Diet. Cell Metabolism, 2012, 15,848860.

12. Ley, R.E., Bäckhed, F., Turnbaugh, P., Lozupone, C.A., et al., Obesity alters gut microbial ecology. Proceedings of the National Academy of Sciences of the United States of America, 2005, 102,11070-11075.

13. Hildebrandt, M.A., Hoffmann, C., Sherrill-Mix, S.A., Keilbaugh, S.A., et al., High-Fat Diet Determines the Composition of the Murine Gut Microbiome Independently of Obesity. Gastroenterology, 2009, 137,17161724.e2.

14. Turnbaugh, P.J., Hamady, M., Yatsunenko, T., Cantarel, B.L., et al., A core gut microbiome in obese and lean twins. Nature, 2009, 457,480-484.

15. Hildebrand, F., Nguyen, T.L., Brinkman, B., Yunta, R.G., et al., Inflammation-associated enterotypes, host genotype, cage and inter-individual effects drive gut microbiota variation in common laboratory mice. Genome Biol, 2013, 14

16. Carlisle, E.M., Poroyko, V., Caplan, M.S., Alverdy, J., et al., Murine gut microbiota and transcriptome are diet dependent. Ann Surg, 2013, 257

17. Clayton, T.A., Baker, D., Lindon, J.C., Everett, J.R., et al., Pharmacometabonomic identification of a significant host-microbiome metabolic interaction affecting human drug metabolism. Proceedings of the National Academy of Sciences, 2009, 106,14728-14733.

18. Dumas, M.E., Kinross, J., and Nicholson, J.K., Metabolic Phenotyping and Systems Biology Approaches to Understanding Metabolic Syndrome and Fatty Liver Disease. Gastroenterology, 2014, 146,46-62.

19. Laghi, L., Picone, G., Cruciani, F., Brigidi, P., et al., Rifaximin Modulates the Vaginal Microbiome and Metabolome in Women Affected by Bacterial Vaginosis. Antimicrobial Agents and Chemotherapy, 2014, 58,3411-3420.

20. de Wit, N.J., Bosch-Vermeulen, H., de Groot, P.J., Hooiveld, G.J., et al., The role of the small intestine in the development of dietary fat-induced obesity and insulin resistance in C57BL/6J mice. BMC Medical Genomics, 2008, 1,1-16. 
21. Benoit, B., Plaisancié, P., Awada, M., Géloën, A., et al., High-fat diet action on adiposity, inflammation, and insulin sensitivity depends on the control low-fat diet. Nutrition Research, 2013, 33,952-960.

22. Neuschwander-Tetri, B.A., Carbohydrate intake and nonalcoholic fatty liver disease. Current Opinion in Clinical Nutrition \& Metabolic Care, 2013, 16,446-452.

23. Roncal-Jimenez, C.A., Lanaspa, M.A., Rivard, C.J., Nakagawa, T., et al., Sucrose induces fatty liver and pancreatic inflammation in male breeder rats independent of excess energy intake. Metabolism, 2011, 60,1259-1270.

24. Tilg, H. and Moschen, A.R., Evolution of inflammation in nonalcoholic fatty liver disease: The multiple parallel hits hypothesis. Hepatology, 2010, 52,1836-1846.

25. McGarry, J.D., Banting Lecture 2001: Dysregulation of Fatty Acid Metabolism in the Etiology of Type 2 Diabetes. Diabetes, 2002, 51,7-18.

26. Houtkooper, R.H., Argmann, C., Houten, S.M., Cantó, C., et al., The metabolic footprint of aging in mice. Scientific Reports, 2011, 1,134.

27. Buchner, D.A., Yazbek, S.N., Solinas, P., Burrage, L.C., et al., Increased Mitochondrial Oxidative Phosphorylation in the Liver Is Associated With Obesity and Insulin Resistance. Obesity, 2011, 19,917-924.

28. Satapati, S., Sunny, N.E., Kucejova, B., Fu, X., et al., Elevated TCA cycle function in the pathology of dietinduced hepatic insulin resistance and fatty liver. Journal of Lipid Research, 2012, 53,1080-1092.

29. Bratic, I. and Trifunovic, A., Mitochondrial energy metabolism and ageing. Biochimica et Biophysica Acta (BBA) - Bioenergetics, 2010, 1797,961-967.

30. Muoio, Deborah M., Metabolic Inflexibility: When Mitochondrial Indecision Leads to Metabolic Gridlock. Cell, 2014, 159,1253-1262.

31. Stefan, N. and Häring, H.-U., The Metabolically Benign and Malignant Fatty Liver. Diabetes, 2011, 60,20112017.

32. Berryman, D.E., Glad, C.A.M., List, E.O., and Johannsson, G., The GH/IGF-1 axis in obesity: pathophysiology and therapeutic considerations. 2013, 9,346-356.

33. Floreani, A., Liver Diseases in the Elderly: An Update. Dig Dis, 2007, 25,138-143.

34. Mariat, D., Firmesse, O., Levenez, F., Guimarăes, V., et al., The Firmicutes/Bacteroidetes ratio of the human microbiota changes with age. BMC Microbiology, 2009, 9,1-6.

35. Bischoff, S.C., Microbiota and aging. Current opinion in clinical nutrition and metabolic care, 2016, 19,26-30.

36. O'Toole, P.W. and Jeffery, I.B., Gut microbiota and aging. Science, 2015, 350,1214-1215.

37. Hyotylainen, T., Jerby, L., Petaja, E.M., Mattila, I., et al., Genome-scale study reveals reduced metabolic adaptability in patients with non-alcoholic fatty liver disease. Nat Commun, 2016, 7.

38. van Ommen, B., van der Greef, J., Ordovas, J.M., and Daniel, H., Phenotypic flexibility as key factor in the human nutrition and health relationship. Genes \& Nutrition, 2014, 9,1-9.

39. Reznick, R.M., Zong, H., Li, J., Morino, K., et al., Aging-Associated Reductions in AMP-Activated Protein Kinase Activity and Mitochondrial Biogenesis. Cell Metabolism, 2007, 5,151-156.

40. Li, Y., Xu, S., Mihaylova, M.M., Zheng, B., et al., AMPK Phosphorylates and Inhibits SREBP Activity to Attenuate Hepatic Steatosis and Atherosclerosis in Diet-Induced Insulin-Resistant Mice. Cell Metabolism, 2011, 13,376-388.

41. Ieta, K., Ojima, E., Tanaka, F., Nakamura, Y., et al., Identification of overexpressed genes in hepatocellular carcinoma, with special reference to ubiquitin-conjugating enzyme E2C gene expression. Int. J. Cancer, 2007, 121,33-38. 
42. Xie, C., Powell, C., Yao, M., Wu, J., et al., Ubiquitin-conjugating enzyme E2C: A potential cancer biomarker. The International Journal of Biochemistry \& Cell Biology, 2014, 47,113-117.

43. Hao, Z., Zhang, H., and Cowell, J., Ubiquitin-conjugating enzyme UBE2C: molecular biology, role in tumorigenesis, and potential as a biomarker. Tumor Biology, 2012, 33,723-730.

44. Harvie, M., Wright, C., Pegington, M., McMullan, D., et al., The effect of intermittent energy and carbohydrate restriction v. daily energy restriction on weight loss and metabolic disease risk markers in overweight women. The British journal of nutrition, 2013, 110,1534-1547.

45. Harvie, M.N., Pegington, M., Mattson, M.P., Frystyk, J., et al., The effects of intermittent or continuous energy restriction on weight loss and metabolic disease risk markers: a randomized trial in young overweight women. Int J Obes (Lond), 2011, 35,714-727.

46. Francois, M.E., Baldi, J.C., Manning, P.J., Lucas, S.J.E., et al., 'Exercise snacks' before meals: a novel strategy to improve glycaemic control in individuals with insulin resistance. Diabetologia, 2014, 57,14371445.

47. Bergouignan, A., Antoun, E., Momken, I., Schoeller, D.A., et al., Effect of contrasted levels of habitual physical activity on metabolic flexibility. Journal of Applied Physiology, 2013, 114,371-379.

48. Koves, T.R., Sparks, L.M., Kovalik, J.P., Mosedale, M., et al., PPARy coactivator-1 a contributes to exerciseinduced regulation of intramuscular lipid droplet programming in mice and humans. Journal of Lipid Research, 2013, 54,522-534.

49. van de Rest, 0., Schutte, B.A., Deelen, J., Stassen, S.A., et al., Metabolic effects of a 13-weeks lifestyle intervention in older adults: The Growing Old Together Study. Aging (Albany NY), 2016, 8,111.

50. Brandhorst, S., Choi, In Y., Wei, M., Cheng, Chia W., et al., A Periodic Diet that Mimics Fasting Promotes Multi-System Regeneration, Enhanced Cognitive Performance, and Healthspan. Cell Metabolism, 2015, 22,86-99.

51. Varady, K.A., Roohk, D.J., Loe, Y.C., McEvoy-Hein, B.K., et al., Effects of modified alternate-day fasting regimens on adipocyte size, triglyceride metabolism, and plasma adiponectin levels in mice. Journal of Lipid Research, 2007, 48,2212-2219.

52. Anson, R.M., Guo, Z., de Cabo, R., lyun, T., et al., Intermittent fasting dissociates beneficial effects of dietary restriction on glucose metabolism and neuronal resistance to injury from calorie intake. Proceedings of the National Academy of Sciences, 2003, 100,6216-6220.

53. Seimon, R.V., Shi, Y.-C., Slack, K., Lee, K., et al., Intermittent Moderate Energy Restriction Improves Weight Loss Efficiency in Diet-Induced Obese Mice. PLoS ONE, 2016, 11 ,e0145157.

54. Cerqueira, F.M., da Cunha, F.M., Caldeira da Silva, C.C., Chausse, B., et al., Long-term intermittent feeding, but not caloric restriction, leads to redox imbalance, insulin receptor nitration, and glucose intolerance. Free Radical Biology and Medicine, 2011, 51,1454-1460.

55. Gallinetti, J., Harputlugil, E., and Mitchell, James R., Amino acid sensing in dietary-restriction-mediated longevity: roles of signal-transducing kinases GCN2 and TOR. Biochemical Journal, 2013, 449,1-10.

56. Mirzaei, H., Suarez, J.A., and Longo, V.D., Protein and amino acid restriction, aging and disease: from yeast to humans. Trends in Endocrinology \& Metabolism, 2014, 25,558-566.

57. Tieland, M., van de Rest, O., Dirks, M.L., van der Zwaluw, N., et al., Protein Supplementation Improves Physical Performance in Frail Elderly People: A Randomized, Double-Blind, Placebo-Controlled Trial. Journal of the American Medical Directors Association, 2012, 13,720-726.

58. Tieland, M., Dirks, M.L., van der Zwaluw, N., Verdijk, L.B., et al., Protein Supplementation Increases Muscle Mass Gain During Prolonged Resistance-Type Exercise Training in Frail Elderly People: A Randomized, Double-Blind, Placebo-Controlled Trial. Journal of the American Medical Directors Association, 2012, 13,713-719. 
59. de Cabo, R., Carmona-Gutierrez, D., Bernier, M., Hall, Michael N., et al., The Search for Antiaging Interventions: From Elixirs to Fasting Regimens. Cell, 2014, 157,1515-1526.

60. Longo, V.D., Antebi, A., Bartke, A., Barzilai, N., et al., Interventions to Slow Aging in Humans: Are We Ready? Aging Cell, 2015, 14,497-510.

61. Martin, B., Pearson, M., Kebejian, L., Golden, E., et al., Sex-Dependent Metabolic, Neuroendocrine, and Cognitive Responses to Dietary Energy Restriction and Excess. Endocrinology, 2007, 148,4318-4333.

62. Browning, J.D., Baxter, J., Satapati, S., and Burgess, S.C., The effect of short-term fasting on liver and skeletal muscle lipid, glucose, and energy metabolism in healthy women and men. Journal of lipid research, 2012, 53,577-586.

63. Stubbins, R.E., Holcomb, V.B., Hong, J., and Núñez, N.P., Estrogen modulates abdominal adiposity and protects female mice from obesity and impaired glucose tolerance. European Journal of Nutrition, 2012 , 51,861-870.

64. Fontana, L. and Klein, S., Aging, adiposity, and calorie restriction. JAMA, 2007, 297,986-994. 

Increasing life expectancy in the past decades has led to the emergence of age-related chronic diseases and disabilities. A deeper understanding in the molecular events of the aging process is essential to provide evidence-based guidance how lifestyle interventions will be more efficient in delaying age-related disease phenotypes. Calorie restriction (CR) is by far the best nutritional strategy to achieve longevity in animal models. Although potentially also effective for humans, most people experience this rigorous diet as not feasible. To search for a practicable alternative we explored, using a C57BL/6J mice cohort, the effects of intermittent (INT) diet, a weekly alternating diet regimen between $40 \mathrm{E} \% \mathrm{CR}$ and ad libitum medium-fat feeding. We hypothesized that the weekly fluctuating energy availability provides beneficial challenges to the body.

In this thesis we focused on the effects induced by the INT diet on the liver, the central metabolic organ in the body. Non-alcoholic fatty liver disease (NAFLD) is the most common liver disease that develops with age and is considered as the hepatic phenotype of the metabolic syndrome. NAFLD is a disease that develops slowly over the years; its prevalence has been shown to increase at old age (>60 years). In chapter 2 we investigated whether the INT dietary regimen was able to reverse the unfavourable effects of a medium-fat ( $25 \% \mathrm{E}$ fat; MF) diet on the liver and its implication on NAFLD development. We showed that, at the age of 12 months, the INT diet prevented NAFLD development. INT-exposed animals retained healthy physiological features as displayed by continuous exposure to $\mathrm{CR}$; maintenance of glucose tolerance, normal insulin levels and low plasma alanine and aspartate aminotransferases. Furthermore, they did not exhibit signs of hepatic steatosis and fibrosis, indicated by the reduced hepatic TG levels and morphological observations. The results presented in chapter 3 show that, at the age of 24 months, INT-fed mice displayed normal plasma ALT levels, no liver inflammation or fibrosis. These mice, however, display mild steatosis with IHTG levels significantly lower than the MF-exposed mice. To summarize, long-term exposure to a MF diet seriously impaired metabolic homeostasis and was a risk factor for NAFLD development. Applying every-other-week 40E\% CR largely reversed the adverse health effects induced by the MF diet. Although the livers of the INT-exposed mice were still protected for the advanced stages of NAFLD, it is noteworthy that, in the long run, liver fat accumulation still occurred.

The second part of chapter 3 describes the obesity-counteracting effects of the INT diet. Part of the mice that had been exposed to the MF diet till 12 months of age was switched to the INT diet until the age of 24 months. The switch to the INT diet successfully improved glucose clearance, survival and liver health, but failed to improve IHTG levels. Within the diet switch experiment, we also investigated the plasticity of adaptive response to the switch by means of transcriptome analysis. Most of the genes differentially expressed between the INT- and MF-exposed mice ( 95\% of 2,667 genes) switched to the INT-expression profile. There was only a small subset of 148 genes 
which expression levels persistently remained similar to the MF diet-induced expression levels, instead of adapting to INT's expression profile. Pathway analysis pointed out that this subset of 148 genes contains genes involved in lipid and xenobiotic metabolism, with PXR as the strongest upstream regulator. This suggests that MF-induced deregulated PXR activity persistently affects lipid and xenobiotic metabolism in the liver of the old diet switch mice. Therefore, we suggested that, despite the strong improvement of overall and liver-specific phenotypes, these persistently regulated genes might have potentially adverse effects on health.

The adaptive response to the diet switch at an old age was further investigated in chapter $\mathbf{4}$, but then in the reverse order: switching from a healthy to an unhealthy diet. Our results showed that, despite the long-term exposure to CR regimen, mice in the CR-MF group displayed hyperphagia, rapid weight gain, and hepatic steatosis. However, no hepatic fibrosis/injury or alteration in CRimproved survival was observed in the diet switch group. The liver transcriptomic profile of CRMF group largely shifted to a profile similar to the MF-fed animals but leaving $~ 22 \%$ of the 1578 differentially regulated genes between the $\mathrm{CR}$ and MF diet groups comparable with the expression of the life-long CR group. Therefore, although the diet switch was performed at an old age, the CRMF-exposed mice were still able to rapidly gain weight to similar level as life-long MF mice with the same age, but without developing severe liver pathologies.

In chapter 5, the data from the different dietary interventions and age time points were combined to further explore the molecular mechanisms underlying the NAFLD development. Hereby, we focussed our analysis on the association with Fgf21, an emerging non-invasive biomarker for NAFLD. We demonstrated that plasma Fgf21 levels strongly reflected liver fat accumulation, confirming its potential as NAFLD marker. Transcriptomics analysis of the liver was performed and revealed that the link between plasma Fgf21 and IHTG levels was associated with differentially regulated PPARa and NRF2 targets during NAFLD. This suggested that the elevated Fgf21 levels in NAFLD was a measure to maintain homeostasis against the adverse effects of lipotoxicity, oxidative stress and endoplasmic reticulum stress in NAFLD. The PPARa challenge test, which was performed by administrating PPARa agonist Wy-14,643 to the mice, confirmed the dysregulation of PPARa signalling in NAFLD, including the hepatic expression of Fgf21.

To conclude, the results presented in this thesis adds to our understanding the effects of different diets have on genotype-phenotype relationships, which translate into different health states and are essential for identifying healthy aging strategies. We investigated the role of different dietary regimen on the phenotypes of genetically identical mice, particularly on an intermittent (INT) diet, which alternates weekly between the ad libitum medium-fat (MF) and calorie restriction (CR) diet. We found that the INT dietary regimen provided a remarkable protection against the 
severe health outcomes of the long-term medium-fat diet consumption, which may improve life quality by reducing the burden of chronic disease. Although it is too early to conclude that the INT dietary regimen (or modulation of the energy intake) is beneficial and safe to be applied in human population, this study is a proof-of-concept of intervening a chronic overnutrition status with a metabolic challenge of energy stress. Further investigation of this novel dietary regimen is needed to allow it to be safely applied in humans. By switching the diets at a defined time point during the study, we demonstrated that, even at middle and old age, the liver is still a highly flexible organ that rapidly adapts its transcriptional program to the different dietary challenges. We also demonstrated that the strong link between the diet-induced NAFLD and Fgf21 denoted a dysregulation of PPARa signalling pathway during the development of the liver disease. 

"It takes a village to raise a child". The same goes for a thesis. This book that you are currently reading is a piece of (hard) work from a lot of people, who put many efforts on "raising" the PhD student. Now that I feel that I was well-raised through these years, I would like to express my gratitude to my "village" (probably literally a village for Wageningen).

First of all, I would like to start with thanking my supervisor. Wilma, you are a great supervisor and more than that, a mentor. I cannot thank you enough for all your guidance starting from my MSc thesis till my PhD project. I very much appreciate the balance of the independence and the guidance you gave me, I felt them as very effective in my development. I admire your creative ways to see and present things from different angles, I hope I can be as good as you are someday! Thank you for your encouragement, your understanding and your incredible energy. Thank you for everything! I am excited to see your future projects, my best wishes for that!

Michael, thank you for accepting me as your PhD student. The idea with the intermittent diet that you and Wilma started up with is a brilliant one. I am honoured to be a part of it and, although some of the results are not as we expected, I hope it is still a study that you would look back proudly. Thank you for your guidance, especially in the past year after you moved to UK, that you have to spend extra efforts for our meetings in between your family time.

I would like to thank my committee Bas Zwaan, Eline Slagboom, Bert Groen and Ronit ShiriSverdlov for your willingness to assess my thesis and being present at my defence. Bas and Eline, you are a dynamic duo for IDEAL, you have always made the annual meetings inspiring. Thank you for your interest on our study and the encouragement you have given to me.

Carolien, I cannot express how I am really thankful for your support. I would not experience the mice aging study as much fun without you. It is unbelievable how much of our time spent for weighing the food portions for the mice, labelling thousands of eppendorf tubes, crushing the tissues, isolating (and re-isolating) the RNA and DNA. We made fun of how clumsy people like us can work in the lab, but we are probably not that bad! I hope you had as much of fun as I had and you also experience my defence and graduation as a celebration for our project.

Mark, thank you for all your support. I cannot imagine surviving all the analyses without you, especially for the microarray data analysis! Thank you for your willingness to have a look at my manuscripts countlessly and also for your suggestions for improvement. I very much appreciate your encouragement.

My office-mates in 49B, thank you for the great time I experienced, my only wish is that we have had our fun sushi lunches more often. Antwi, thank you for being my paranymph. I admire your coolness and perseverance. It has only been a while since you started your $\mathrm{PhD}$, but you have built a great foundation for your thesis! Ya, we started at about the same time and it was nice to have 
you as a fellow along the way. It is amazing how you juggle your PhD with your cute family. A bit more then you will be ready, good luck with finishing your PhD! Rogier, the source of practical Dutch knowledge for your foreign officemates, I will miss discussing (unimportant) things with you. Thank you for cracking up the other officemates, such an entertaining breaks during my thesis writing days. Shohreh, our mommy in the office, I think every room with PhD students deserves a Shohreh, thank you for the friendship and your great care. Jenny, your family is one of the most gorgeous families I have ever met. Thank you for the microarray analysis and all your help! Karin, I will always remember your amazing homemade pies, they put flavors in my boring bread lunches. Suzanne, it is nice that you joined us every Friday, good luck with finishing your PhD! Sheril, Frits and Jessica, it was such a pleasure to share the office with you. Thank you for the tips in surviving PhD!

Dieuwertje, it was very nice that you joined us in analyzing the colon and the microbiota of the mice aging cohort. You have interesting way to look at the data and you are such a great addition to the study! Thanks for being helpful, especially in working with $\mathrm{R}$ and analyzing the DNA methylation data. Klaske, thank you for your interest in our mice study, which eventually resulted in a collaboration! The daily activity measurement is my favorite addition in the study, it added a straightforward and strong message in our study! I am wishing you all the best with your running and future projects. Rinke, I am grateful that I can come to you for advices on our analysis or rebuttal. Thank you for your help and suggestions, good luck with your future career! Sander, thank you for your support. Your suggestions on our manuscripts are always helpful, thanks for willing to read them! Guido, you are always ready to help others, thank you that I can come to you at any time and also for your interest in our study. Lydia, thank you for your interest in our study. Mechteld and Mieke, thank you for your help around the lab. Diederik, it was fun to hear you practicing your Indonesian with me. Good luck with your studies! Michiel, Jocelijn, and Renger, thank you for your interest in our study. Danielle, Milene, Katja, Jvalini and Nikkie, thank you for the good times and the PhD survival tips! Inge, Juri, Parastoo, Wieneke, Neeraj, Sophie, Lily, Aafke, thank you for the good times and good luck with finishing your PhD! Montserrat, Charlotte and Merel, have fun with your PhD projects!

I would also like to thank all the members of IDEAL consortium. My special thanks go to: Joris, Erik, Marian and Joost. Thank you for your critical look and input for the manuscripts. Elmar and Wouter, thank you for your advices! Bologna team: Vincenzo, Luca, Stefano and Claudio, thank you for your interest in our study and of course for analyzing the plasma glycomics and the urine metabolomics!

Miriam, it was great to collaborate with you and Klaske. The scheduling of the sacrifice became more tricky with the additional analyses that you performed. But, the more the merrier, having you 
there added more fun and the analyses are certainly worth the efforts! Aswin, thank you for your immense help on our liver sections!

Judith, Lisette, Romy, Rene, Bert, thank you for making the animal study went as it was and for all your support!

To the students who performed their thesis projects on the IDEAL mice study: Dennis, Wenny, Aran, Jeroen, Melissa, Paulien, Timme, Albert, Chantal, Jenkau, Evi, Dila, Chen, Laurie. For those who still got the chance to meet the mice personally: thank you for your willingness to help with the study. And to all of you, thank you for your interest and dedication on thesis projects! I am aware that not every projects went smoothly, but I very much appreciate all your contribution to our knowledge on the study!

Karen and Steisi, when we hanged around in fateta canteen or our student rooms in Darmaga, who knew that years later we went to the Netherlands around the same time and obtained our master degree there? I am thankful that our paths always cross, this journey is lovelier with you in it. Cecile, Sylvia and Theresia, you have always been a part of my journey (although lately, virtually), starting from high school. We are all in different places, yet we can still share anything. Thank you for your love and support! Kenzie, you are a hidden surprise in the Netherlands. We were only a friend of a friend, until the day that I visited you in Groningen. Thank you for all your advices and encouragement in finishing my PhD!

Bu Maggy, my bachelor thesis supervisor. You are the initial inspiration of where I am now. Thank you for being so supportive and tirelessly provided me with recommendation letters, so that I could pursue further study.

Mbak Susan and Mbak Irene, both alumnae of Wageningen University and my former bosses in Nutrifood Indonesia. Thank you for introducing me to Wageningen University, you are such great role models!

Mijn lieve schoonfamilie; papa, mama, oma, Mark en natuurlijk alle andere naaste familieleden. Bedankt voor jullie liefde, warmte, oprechte zorg en jullie onvoorwaardelijke steun. Ik heb me vanaf het begin af aan welkom gevoeld bij jullie. Ik kan niet beschrijven hoe blij ik met jullie ben en hoe fijn ik het vind dat jullie er altijd voor me zijn. Mijn bijzondere dank aan Astrid, voor het proeflezen van mijn introductie en discussie, tijdens je eigen drukke periode!

To my family: my parents papi mami, and my sister ci Imelda. Saying thank you will not be enough to express how grateful I am to have been supported and encouraged by you through every stage in my life. The journey that I took was probably not so common, but I hope you can be proud of what I accomplished with this dissertation. You are the reason why I would like to contribute to the aging research. My wish is that this little contribution of mine may someday somehow lead to 
a longer healthy life for everyone, which means you can spend more time with your loved ones.

Dave, my beloved "partner" (as defined by Otto to Carrie)! I love you "more than sharks love blood". I would not know how to complete my PhD without your support and encouragement. There are so many things to thank you, but most of all: your understanding that in this 4.5 years a large part of my time had to be invested in my study and you had to make some sacrifices as well for me. Now I am finally there, it is your time to soar! I am looking forward to our next challenges! 


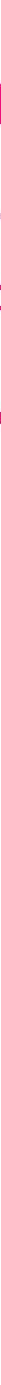

About the Author 

Fenni Rusli was born on February 1, 1984 in Jakarta, Indonesia. She did her bachelor study on Food Science and Technology in Bogor Agricultural University, with a focus on microbiology and biochemistry. She graduated in 2006 as the best graduate in the Faculty of Agricultural Technology. At the end of her bachelor study, in a team of three she won the first prize in the $19^{\text {th }}$ Annual Student Creativity Competition by the Indonesian Ministry of Education. Her bachelor thesis on restriction enzymes also won the third prize of the Young Indonesian Researcher Award by the Indonesian Institute of Science. She proceeded to work in Nutrifood Indonesia as a research and development staff and later on in the Nutrifood Research Center division. In 2009 she received the Netherlands Fellowship Programme and continued with master study on Nutrition and Health in Wageningen University. In 2010 she did her first master thesis focused on age-related DNA methylation changes in the small intestines. at Nutrition, Metabolism and Genomics under supervision of Dr. W.T. Steegenga. She continued with a second master thesis at Genetics lab guided by Prof. Dr. B.J. Zwaan and Dr. A.J.M. Debets, studying the phenotypic plasticity of the fungus Podospora anserina in response to calorie restriction. During her master study, she was also a secretary for the Indonesian Student Association in Wageningen. In 2011 she started a PhD project at the Nutrition, Metabolism and Genomics group under the guidance of Dr. W.T. Steegenga and Prof. Dr. M. Müller. Her PhD project is a part of "Integrated research on developmental determinants of ageing and longevity" (IDEAL) project, which was funded by the EU. The results of the PhD project "Diet-induced phenotypic plasticity during aging" are described in this thesis. 

F. Rusli, M.V. Boekschoten, A. A. Zubia, C. Lute, M. Müller \& W.T. Steegenga, "A weekly alternating diet between caloric restriction and medium-fat protects the liver from fatty liver development in middle-aged C57BL/6J mice." Molecular Nutrition \& Food Research, 2015.

F. Rusli, J. Deelen, E. Andriyani, M.V. Boekschoten, C. Lute, E.B. van den Akker, M. Müller, M. Beekman, W.T. Steegenga. "Fibroblast growth factor 21 reflects liver fat accumulation and dysregulation of signalling pathways in the liver of C57BL/6J mice." Scientific Reports, 2016.

F. Rusli, C. Lute, M.V. Boekschoten, M. van Dijk, K. van Norren, A.L. Menke, M. Müller, W.T. Steegenga. "Intermittent calorie restriction largely counteracts the adverse health effects of a medium-fat diet in aging C57BL/6J mice." Submitted.

F. Rusli, M.V. Boekschoten, V. Borelli, C. Sun, C. Lute, A.L. Menke, J. van den Heuvel, S. Salvioli, C. Franceschi, M. Müller, W.T. Steegenga. "Plasticity of life-long calorie restricted C57BL/6J mice in adapting to a medium-fat diet intervention at old age." Submitted.

K. van Norren, F. Rusli, M. van Dijk, C. Lute, J. Nagel, F.J. Dijk, J. Dwarkasing, M.V. Boekschoten, Y. Luiking, R.F. Witkamp, M. Müller \& W.T. Steegenga. "Behavioural changes are a major contributing factor in the reduction of sarcopenia in caloric-restricted ageing mice." Journal of Cachexia, Sarcopenia and Muscle, 2015.

D. Kok, F. Rusli, A. Lopez Nadal, C. Lute, L. Laghi, S. Salvioli, F. Capozzi, C. Franceschi, E. Kampman, H. Smidt, J. Vervoort, M.V. Boekschoten, M. Müller and W.T. Steegenga. “Lifelong caloric restriction determines comprehensive molecular signatures related to colonic health in aging C57BL/6J mice." In preparation. 



\section{Discipline specific activities}

- "Why and how we age", Leyden Academy on Vitality and Ageing summer course (Leiden, The Netherlands, 2011)

- Science camp GRK1482 Technical University Munich (Seeon, Germany, 2013)

- Dutch Liver Retreat (Spier, The Netherlands, 2014)

- Epigenesis and Epigenetics (Wageningen, The Netherlands, 2014)

- Weighted gene co-expression network analysis (WGCNA) workshop (Leiden, The Netherlands, 2014)

- Liver metabolism and non-alcoholic fatty liver disease (Whistler, Canada, 2015)

- IDEAL Dissemination Meeting (Leiden, The Netherlands, 2015)

- IDEAL Annual Meeting (Oegstgeest, The Netherlands, 2012; Paris, France, 2013; Leiden, The Netherlands, 2014; Bologna, Italy, 2015)

- NWO Voedingsdagen/ Nutritional Science Days (Deurne, The Netherlands, 2011, 2012, 2013, 2014)

\section{General courses}

- Laboratory Animal Science (2012)

- PhD Week (2012)

- Scientific Writing (2014)

- Career Perspective (2014)

\section{Optional activities}

- PhD study tour (Australia, 2013)

- NMG-Pharma lab meetings (weekly, 2011-2016)

- IDEAL Work Package 4 discussion (monthly, 2012)

- Epigenetics journal club (monthly, 2014-2015) 


\section{Colophon}

The research presented in this thesis has received full funding from the European Union's Seventh Framework Programme (FP7/2007-2011) IDEAL-aging under grant agreement no. 259679.

Financial support from Wageningen University for printing this thesis is gratefully acknowledged.

Design and layout : Briyan B. Hendro (briyan.2209@yahoo.com)

Printed by : Digiforce

(c) Fenni Rusli, 2016 